\title{
Spatial Statistics for Predicting Flow through a Rock Fracture
}

\author{
Kevin Jerome Coakley \\ Earth Sciences Division \\ Lawrence Berkeley Laboratory \\ 1 Cyclotron Road \\ Berkeley, Califomia 94720
}

\section{A DISSERTATION \\ SUBMITTED TO THE DEPARTMENT OF STATISTICS AND THE COMMITTEE ON GRADUATE STUDIES OF STANFORD UNIVERSITY \\ IN PARTIAL FULFILLMENT OF THE REQUIREMENTS FOR THE DEGREE OF DOCTOR OF PHILOSOPHY}

March 1989

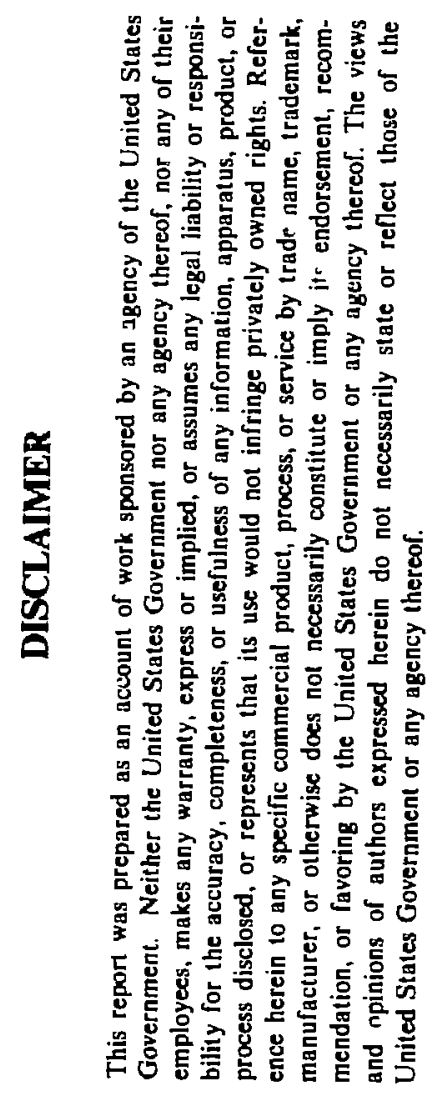

The United States Deparment of Energy has the right to use this thesis for any purpose whatsoever including the right to seproduce all or any part thereof.

This work was supported by the Director, Office of Civilian Radioactive Wastc Management, Office of Geologic Repositories. Transportation and Technology Division, of the U.S. Department of Energy under Contract No. DE-AC03-76SF00098.

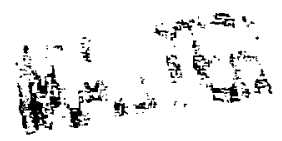


() Copyright by Kevin J. Coakley 1989

All Rights Reserved

The Goverment reserves for itself and others acting on its behalf a royalty free, nonexclusive, irrevocable, world-wide license for governmental purposes to publish, distribute, translate, duplicate, exhibit, and perform any such data opyrighted by the contractor. 


\section{ABSTRACT}

Fluid flow through a single rock fracture depends on the shape of the space between the upper and lower pieces of rock which define the fracture. In this thesis, the normalized flow through a fracture, i.e. the equivalent permeability of a fracture, is predicted in terms of spatial statistics computed from the arrangement of voids, i.e. open spaces, and contact areas within the fracture. Patterns of voids and contact areas, with complexity typical of experimental data, are simulated by clipping a correlated Gaussian process defined on a $N$ by $N$ pixel square region. The voids have constant aperture; the distance between the upper and lower surfaces which define the fracture is either zero or a constant. Local flow is assumed to be proportional to local aperture cubed times local pressure gradient. The flow through a pattern of voids and contact areas is solved using a finite-difference method.

After solving for the flow through simulated 10 by 10 and 30 by 30 pixel pattems of voids and contact areas, a model to predict equivalent permeability is developed. The first model is for patterns with $80 \%$ voids where all voids have the same aperture. The equivalent permeability of a pattern is predicted in terms of spatial statistics computed from the arrangement of voids and contact areas within the pattem. Four spatial statistics are examined. The change point statistic measures how often adjacent pixels altemate from void to contact area (or vice versa) in the rows of the patterns which are parallel to the overall flow direction. The dispersion statistics describe how widely dispersed the voids are within the rows and columns of the pattem. For instance, the row dispersion statistic takes its minimum value if each row in the pattern has the same number of voids. The final statistic is based on erosion (Serra, 1982) transformations of the pattem and measures a controlling flow path width in between contact area features.

The 0.80 void fraction model is extended to patterns with other void fractions. The extended model accurately predicted the equivalent permeability of simulated 
patterns ranging in scale from 30 by 30 pixels to 128 by 128 pixels. It is expected that the prediction model will work for even larger simulated patterns from the same simulation model. How well the model works for larger patterns simulated using other models is an open question. However, in general, the extended model accurately predicted the equivalent permeability of some patterns simulated differently including some simulated using fractal ideas (Mandelbrot, 1983). Limitations of the model are noted.

Using the prediction model, which is good for patterns where voids have constant aperture, a lower bound for the equivalent permeability of a variable aperture pattern is given.

In addition to predicting the flow through a particular simulated pattern, a lower bound for the variability of equivalent permeability for an ensemble of simulated patterns is given. To get the lower bound, both the Power Average Model (Journel et al., 1986) and Percolation Theory (Broadbent and Hammersley, 1957) are used to relate the void fraction of a pattern to its expected equivalent permeability. 


\section{ACKNOWLEDGEMENTS}

I wish to thank both the Statistics Department of Stanford University and Jane Long of the Earth Sciences Division of Lawrence Berkeley Laboratory for supporting my graduate studies and thesis research. Working at LBL was a rewarding personal and scientific experience. I benefited greatly from the opportunity to work on important problems in a stimulating and friendly environment. I thank Jane Long for motivating my research and for her keen insights. Paul Switzer has been a great advisor, he knows what is important.

During my thesis research, I benefited from conversations with Daniel Billaux, Phillippe Doyen, Kevin Hestir, Aharon Kaptitulnik, Kenzi Karasaki, K. Muralidhar, Larry Myer, Art Owen and Robert Zimmerman.

Lastly, I wish to recognize the support of my parents and friends. 


\section{Table of Contents}

List of Figures ...................................................................................... viii

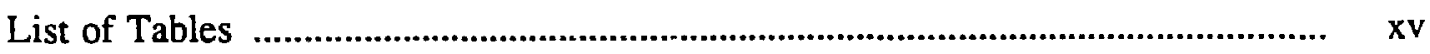

Nomenclature .................................................................................................. xviii

1. Introduction ..............................................................................................

2. Literature Review ..................................................................................... 11

3. Simulation Model ............................................................................................ 20

4. Flow Model ................................................................................................... 35

5. Spatial Statistics for Flow ….......................................................................... 53

5.1 Mathematical Properties of Spatial Statistics ............................................... 54

5.2 Change Point Statistic ............................................................................... 58

5.3 Row and Column Dispersion Statistics ....................................................... 70

5.4 Erosion Statistic ......................................................................................... 84

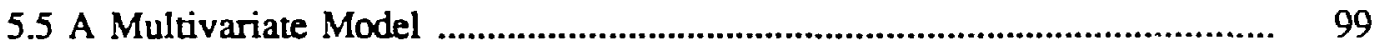

6. Extension to Other Void Fractions .................................................................... 109

6.1 Extended Models .................................................................................. 110

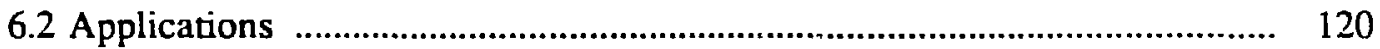

7. Ensemble Variability ......................................................................................

8. Variable Aperture Patterns ……..................................................................... 159

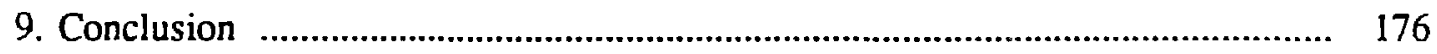

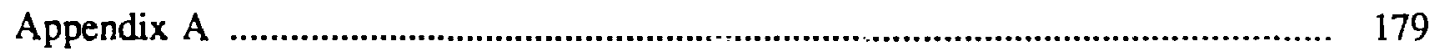

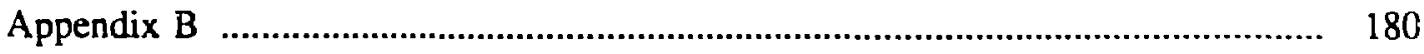

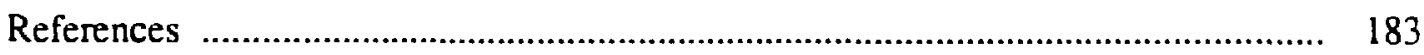




\section{LIST OF FIGURES}

Figure 1.1 A simulated pattem of voids(black) and contact areas(white).

Figure 1.2 For flow in the $x$ direction, the contact area feature

(white) in Pattern A blocks flow more efficiently than the contact area feature in Pattern B.

Figure 1.3 Even though both Patterns $C$ and $D$ have contact area features with the same shape, a pressure drop in the $x$ direction leads to less flow through Pattern $C$ than through Pattern D.

Figure 3.1 Partern 128a is an observed 128 by 1.28 pixel pattern of voids and contact areas within a single fracture. The data is from a metal injection experiment. Voids are black and contact areas are white. Each pixel length is $0.21 \mathrm{~mm}$. $18.3 \%$ contact area.

Figure 3.2 Simulated patterns 128b, 128c, 128d. All have 18.3\% contact area. Scale is the same as in Figure 3.1.

Figure 3.3 Correlograms of the observed pattern (128a) and the simulated patterns (128b, 128c, 128d).

Figure 3.4 Exponential fit $e^{-\lambda r}$ to correlogram. $\lambda=0.505 . r$ is lag in pixel lengths.

Figure 3.5 Two contact area pixels which share a side are part of the same contact area feature. As an illustration, the pattern on the far left has one contact area feature. Each of the other two patterns have two fearures.

Figure 3.6 Histogram and cumulative histogram for contact area feature sizes of observed pattern (128a) and simulated patterns (128b, 128c, 128d).

Figure 3.7 Simulated 10 by 10 pixel patterns with $80 \%$ voids. Patterns result from clipping a correlated Gaussian process. Top row has patterns $10 \mathrm{a}, 10 \mathrm{~b}$ and $10 \mathrm{c}$. Second row has $10 \mathrm{~d}, 10 \mathrm{e}$ and $10 \mathrm{f}$. Third row has $10 \mathrm{~g}, 10 \mathrm{~h}$ and $10 \mathrm{i}$. Bottom row has $10 \mathrm{j}$. 
Figure 3.8 Simulated 30 by 30 pixel patterns with $80 \%$ voids. Patterns result from clipping a correlated Gaussian process. Top row has patterns 30a, 30b, 30c, and 30d. Second row has $30 \mathrm{e}, 30 \mathrm{f}, 30 \mathrm{~g}$ and $30 \mathrm{~h}$. Third row has $30 \mathrm{i}, 30 \mathrm{j}, 30 \mathrm{k}$ and 301 . Bottom row has $30 \mathrm{~m}$.

Figure 4.1 When a uniform pressure gradient in the $x$ direction forces a fluid between parallel plates, the Navier-Stokes equations predict a paraboiic fluid velocity profile between the plates and that total flow is proportional to aperture cubed $\left(d^{3}\right)$.

Figure 4.2 Boundary conditions for solving for flow due to a pressure drop in the $x$ direction.

Figure 4.3 A 10 by $1 \mathrm{C}$ pixel pattern with $80 \%$ voids. Black pixels are voids with constant aperture. White pixels are contact areas. For flow in the $x$ direction, equivalent permeability is 0.80 . For flow in the $y$ direction, equivalent permeability is 0.0 .

Figure 4.4 In the finite difference solution for flow, pressure is solved for at nodes of a mesh which covers the fracture region. The nodes are where the lines intersect.

Figure $4.5 \quad$ For a 10 by 10 pixel pattern, a finite difference mesh of size 10 by 10 would assign one node to each pixel. A 20 by 20 mesh would assign 4 nodes per pixel. A 30 by 30 mesh would assign 9 nodes per pixel. Above, from left to right, we show how a pixel in a 10 by 10 pixel pattern would be assigned nodes for the three meshes mentioned.

Figure 4.6 Each node in the mesh, except for boundary nodes, has four neighboring nodes. In the finite difference model, the flow between nodes is proportional to the pressure differences between the neighboring nodes times the harmonic average of aperture cubed.

Figure 4.7 Computed equivalent permeability versus mesh size for pattern 10e. Pressure drop applied in $y$ direction. Mesh size is $\mathrm{k}$ by $\mathrm{k}$. $\mathrm{X}$ axis is $\mathrm{k}$.

Figure 4.8 Computed equivalent permeability versus mesh size for pattern 30b. Pressure drop applied in $x$ direction. Mesh size is $\mathrm{k}$ by $\mathrm{k}$. $\mathrm{X}$ axis is $\mathrm{k}$. 
Figure 5.1.1 Even though pattems $\mathrm{A}$ and $\mathrm{B}$ are rotated version of one another, for flow in the $x$ direction, the contact area feature in pattern A blocks flow more efficiently than the contact area feature in pattern B. Since flow depends on the direction of the pressure drop, any spatial statistic computed from a pattern must also depend on the direction of the applied pressure drop.

Figure 5.1.2 A basic pattem (10 by 10 pixels), its 2 by 2 replication ( 20 by 20 pixels) and its 8 by 8 replication ( 80 by 80 pixels). Spatial statistics which are determinisitic replication invariant are the same for all three patterns.

Figure 5.2.1 In the rows which run in the $x$ direction, there are no alternations between contact areas and voids or vice versa. In the columns which run in the $y$ direction, there are two altemations (change points) per column.

Figure 5.2.2 Equivalent permeability versus $N C P$ for 10 by 10 pixel and 30 by 30 pixel simulated pattems with $80 \%$ voids. Straight line is predicted equivalent permeability according to NCP model.

Figure 5.2.3 For patterns $\mathrm{C}$ and $\mathrm{D}$, for a pressure drop in the $x$ direction, NCP is the same even though the relative locations of the contact area features are different for each pattern.

Figure 5.3.1 Equivalent permeability versus column dispersion statistic (NSIGC) for 10 by 10 pixel and 30 by 30 pixel simulated pattems with $80 \%$ voids.

Figure 5.j.2 Equivalent permeabilie's versus row dispersion statistic (NSIGR ) for 10 by 10 pixel and 30 by 30 pixei simulated patterns with $80 \%$ voids.

Figure 5.3.3 Row dispersion statistic (NSIGR) versus column dispersion statistic (NSIGC) for 10 by 10 pixel and 30 by 30 pixel patterns. Pattems with zero equivalent permeability are not plotted.

Figure 5.3.4 Predicted equivalent permeability according to dispersion statistic model yersus actual equivalent permeability for the 10 by 10 pixel and 30 by 30 pixel pattems with $80 \%$ void fractions.

Figure 5.4.1 Trapezoidal pattern. Void region is black. Contact areas are white. 
Figure 5.4.2 Fin approximation for equivalent permeability of trapezoidal patterns with $50 \%$ contact area.

Figure 5.4.3 Original 10 by 10 pixel pattern and eroded versions of it for a pressure drop in the $y$ direction. BREAK is three because it takes three erosions to disconnect the inflow and outflow boundaries.

Figure 5.4.4 Replication family of patterns for which NBREAK is invariant.

Figure 5.4.5 Replication family for which NBREAK is not invariant.

Figure 5.4.6 Equivalent permeability versus NBREAK for 10 by 10 pixel and 30 by 30 pixei simulated patterns with $80 \%$ voids. Predicted equivalent permeability according to NBREAK model is plotted as a line.

Figure 5.5.1 Scatterplots of spatial statistics of 10 by 10 pixel and 30 by 30 pixel simulated patterns with $80 \%$ voids.

Figure 5.5.2 Predicted equivalent permeability according to three 107 stutistic (NCP, NSIGC and NSIGR) model versus actual equivalent permeability for 10 by 10 pixel and 30 by 30 pixel simulated patterns.

Figure 6.1.1 From the top, simulated patterns $30 \mathrm{~m}, 30 \mathrm{n}, 30 \mathrm{o}, 30 \mathrm{p}, 30 \mathrm{q}$. Void fractions are $0.60,0.70,0.80,0.90$ and 0.95 . Voids have constant aperture.

Figure 6.1.2 From the top, simulated patterns 30r, 30s, 30̂, and 30u. Void fractions are $0.65,0.75,0.85$, and 0.95 . Voids have constant aperture.

Figure 6.1.3 In clockwise order, from upper left, simulated pattems $64 a, 64 b, 64 c$ and $64 d$. Voids have constant aperture.

Figure 6.1.4 Predicted equivalent permeability according to Model A (three statistic extended model) versus actual equivalent permeability for 30 by 30 pixel and 64 by 64 pixel pattems with variable void fractions. Void fractions are indicated on the plot.

Figure 6.1.5 Predicted equivalent permeability according to Model B (NCP extended model) versus actual equivalent permeability for 30 by 30 pixel and 64 by 64 pixel patterns with variable void fractions. Yoid fractions are indicated on the plot. 
Figure 6.1.6 Model A and Model B predicted equivalent permeability for a family of patterns. The same 30 by 30 pixel iealization of a correlated Gaussian defined on a 30 by 30 pixel square region, is clipped at different levels to get different members of the family. Computed equivalent at void fractions of $0.60,0.70,0.80,0.90$ and 0.95 is plotted for comparison.

Figure 6.2.1 Model A prediction for equivalent permeability versus actual equivalent permeability for observed (128a) and simulated (1285, 128s, 128d) 128 by 128 pixel patterns.

Figure 6.2.2 Model B prediction for equivalent permeability versus actual equivaient permeability for observed (128a) and simulated (128b, 128c, 128d) 128 by 128 pixel patterns.

Figure 6.2.3 From the top, simulated 30 by 30 pixel patterns $30 \mathrm{v}$, $30 \mathrm{w}, 30 \mathrm{x}, 30 \mathrm{y}$. Void fractions are $0.65,0.75,085$ and 0.95 . Voids have constant aperture. Moving average filter parameter ( $x)$ is 0.25 .

Figure 6.2.4 From the top, simulated 30 by 30 pixel patterns $30 \mathrm{z}$, $30 \mathrm{aa}, 30 \mathrm{bb}, 30 \mathrm{cc}$. Void fractions are $0.65,0.75,0.85$ and 0.95 . Voids have constant aperture. Moving average filter parameter $(\boldsymbol{k})$ is 1.25 .

Figure 6.2.5 Model A predicted equivalent permeability versus actual equivalent permeability for simulated 30 by 30 pixel patterns shown in Figures 6.2.3 and 6.2.4. For each pattern, the moving average filter parameter $(\kappa)$ was 0.25 or 1.25 .

Figure 6.2.6 Model B predicted equivalent permeability versus actual equivalent permeability for simulated 30 by 30 pixel patterns shown in Figures 6.2.3 and 6.2.4. For each pattern, the moving average filter parameter $(\kappa)$ was 0.25 or 1.25 .

Figure 6.2.7 The buildup of the Sierpinski carpet fractal. The $n=\infty$ member of the above sequence is a fractal. Figure is from Stauffer (1985).

Figure 6.2.8 Simulated patterns $64 \mathrm{fa}$ and $30 \mathrm{fa}$. These patterns are simulated using fractal ideas. Black pixels are voids and white pixels are contact. Void fractions are 0.667 and 0.631 . 
Figure 6.2.9 Simulated patterns $64 \mathrm{fb}$ and $30 \mathrm{fb}$. These patterns are simulated using fractal ideas. Black pixels are voids and white pixels are contact. Void fractions are 0.819 and 0.788 .

Figure 6.2.10 Simulated patterns 64fc and 64fd. These patterns are simulated using fractal ideas. Black pixels are voids and white pixels are contact. Void fractions are 0.865 and 0.837 .

Figure 6.2.11 Model A prediction for equ:yalent permeability versus actual equivalent permeability for observed (128a) and simulated (128b, 128c, 128d) 128 by 128 pixel patterns.

Figure 6.2.12 Model A predicted equivalent permeability versus actual equivalent permeability for 30 by 30 and $6+$ by 64 pixel patterns simulated using fractal ideas.

Figure 6.2.13 Model B predicted equivalent permeability versus actual 142 equivalent permeability for 30 by 30 and 64 by 64 pixel patterns simulated using fractal ideas.

Figure 6.2.14 Magnitude of the prediction error $\left(\left|K_{\text {eq }}-\hat{K}_{\text {eq }}\right|\right)$ for Model $A$ and Model $B$ for the 30 by 30 and 64 by 64 pixei patterns simulated using fractal ideas.

Figure 7.1 Power average model fit $\left(\hat{K}_{\star 4}=p^{3.43}\right)$ and actual equivalent permeability for 64 by 64 pixel patterns (Table 6.1.4). Void fraction denoted by $p$.

Figure 8.1 Pattern of correlated Gaussians. Darker pixels correspond to larger Gaussians.

Figure 8.2 Cumulative aperture histograms for patterns $8 a$ and $8 b$.

Figure 8.3 To get the first lower bound for pattern 8a, all apertures below an adjustable cutoff $\left(b_{c u}\right)$ are set to zero. All apertures above the cutoff are set to the cutoff. Top plot is estimated equivalent permeability $\left(K_{\text {eq }}^{T}\right)$ of transformed pattern versus the fraction $\left(p_{b_{c o u s}}\right)$ of apertures above the adjustable cutoff $b_{c u}$. Middle plot is $b_{c u}{ }^{3}$ versus $p_{b_{c u}}$. Bottom plot is $K_{e q}^{T} b_{c u}{ }^{3}$ versus $p_{\left(b_{c u s}\right)}$. The best lower bound is the maximum of the bottom curve.

Figure 8.4 Same as Figure 8.3 but for pattern $8 \mathrm{~b}$ rather than pattern 8 a. 
Figure 8.5 Variable aperture pattem. Equal apertures appear as same color.

Figure 8.6 Same pattern as in Figure 8.5 except for contact area strip in the middle of the pattern. Equal apertures appear as same color.

Figure 8.7 To get the second lower bound for pattern 8a, all apertures below an adjustable cutoff $\left(b_{c u}\right)$ are set to zero. The rest of the apertures are equated to harmonic average of apertures above the cutoff. From the top, $\left(K_{e q}^{T}\right)$, harmonic average of apertures above the cutoff, and the their product which is the second lower bound. The best lower bound is the maximum of the bottom curve.

Figure 8.8 Same as Figure 8.7 but for pattern $8 \mathrm{~b}$ instead of pattern 8 .

Figure B.1 Approximation and exact expression for standard deviation of void fraction as a function of pattern side length for a simulated pattern with exponential correlogram. Here, correlogram is taken to be $e^{-\lambda r}$ where $r$ is lag in pixel lengths and $\lambda$ is taken to be 0.505 (inverse pixel lengths). 


\section{LIST OF TABLES}

Table 4.1 Dependence of Equivalent Permeability on mesh size for pattern $10 \mathrm{e}(\hat{y})$.

Table 4.2 Equivalent Permeability of the 10 by 10 pixel patterns with $80 \%$ voids.

Table 4.3 Dependence of Equivalent Permeability on mesh size for pattern 30b $(\hat{x})$.

Table $4.4 \quad$ Equivalent Permeability of the 30 by 30 pixel patterns with $80 \%$ voids.

Table 5.2.1 Change point statistics and actual and predicted equivalent permeability for the 10 by 10 pixel patterns with $80 \%$ voids.

Table 5.2.2 Change point statistics and actual and predicted equivalent permeability for the 30 by 30 pixel patterns with $80 \%$ voids.

Table 5.2.3 Fitted values of Change Point statistic model parameter and $R \hat{S E}$.

Table 5.2.4 Fitred model parameter values and $R \hat{S} E$ for inconsistent

Change Point statistic model.

Table 5.3.1 Dispersion statistics, predicted and actual equivalent permeability for 10 by 10 pixel patterns with $80 \%$ voids.

Table 5.3.2 Dispersion statistics, predicted and actual equivalent permeability for 30 by 30 pixel patterns with $80 \%$ voids.

Table 5.3.3 Fitted parameters and root mean square prediction error for dispersion statistic prediction model.

Table 5.3.4 Fitted parameters and root mean square prediction error for inconsistent dispersion statistic prediction model.

Table 5.4.1 Erosion statistics, equivalent permeability and predicted equivalent permeability for 10 by 10 pixel patterns with $80 \%$ voids.

Table 5.4.2 Erosion statistics, equivalent permeability and predicted equivalent permeability for 30 by 30 pixel patterns with $80 \%$ voids.

Table 5.4.3 Fitted parameters for Erosion statistic prediction model. 
Table 5.5.1 Spatial statistics, equivalent permeability and multivariate model prediction for the equivalent permeability of the 10 by 10 pixel patterns with $80 \%$ voids.

Table 5.5.2 Spatial statistics, equivalent permeability and multivariate 103 model prediction for the equivalent permeability of the 30 by 30 pixel patterns with $80 \%$ voids.

Table 5.5.3 Fitted parameters and root mean square prediction error 104 for four statistic prediction model.

Table 5.5.4 Fitted parameters and root mean square prediction error 105 for three statistic prediction model.

Table 5.5.5 Comparison of $R \hat{S E}$ for prediction models for patterns with $80 \%$ voids.

Table 6.1.1 Equivalent permeabi' ity dependence on mesh size for pattern 30n $(\hat{y})$.

Table 6.1.2 Spatial statistics, predicted and actual equivalent permeability for 30 by 30 pixel patterns with variable void fractions.

Table 6.1.3 Equivalent permeability dependence on mesh size for pattern 64a $(\hat{x})$.

Table 6.1.4 Spatial statistics, predicted and actual equivalent permeability for 64 by 64 pixel patterns.

Table 6.1.5 Fitted parameters and root mean square prediction error for extended models.

Table 6.2.1 Equivalent permeability dependence on mesh size for pattern $128 \mathrm{~b}(\hat{y})$.

Table 6.2.2 Predicted and actual equivalent permeabilities for the 128 by 128 pixel patterns.

Table 6.2.3 Comparison of root mean square prediction error and bias of Model A and Model B for 30 by 30,64 by 64 and 128 by 128 pixel patterns from Chapter 3 model.

Table 6.2.4 Predicted and actual equivalent permeability for 30 by 30 pixel simulated patterns with moving average parameter $\kappa=0.25$.

Table 6.2.5 Predicted and actual equivalent permeability for 30 by 30 pixel simulated patterns with moving average parameter $\kappa=1.25$. 
Table 6.2.6 Comparison of $R \hat{S E}$ and bias of Model A and Model B for 30 by 30 pixel patterns simulated using diffe,ent moving average filters.

Table 6.2.7 Predicted and computed equivalent permeability for patterns $64 \mathrm{fa}$ and $30 \mathrm{fa}$.

Table 6.2.8 Predicted and computed equivalent permeability for patterns $64 \mathrm{fb}$ and $30 \mathrm{fb}$.

Table 6.2.9 Predicted and computed equivalent permeability for patterns $64 \mathrm{fc}$ and $64 \mathrm{fd}$.

Table 6.2.10 Predicted and computed equivalent permeability for patterns $64 \mathrm{fd}$, 64fe and 64ff.

Table 6.2.11 Prediction errors for 30 by 30 and 64 by 64 pixel patterns simulated using fractal ideas.

Table 6.2.12 Summary of all the patterns studied to build and test prediction models.

Table 8.1 Equivalent permeability versus mesh size for variable aperture pattem $8 a$.

Table 8.2 Equivalent permeability versus mesh size for variable aperture pattem $8 b$.

Table 8.3 First lower bound for the equivalent permeability of variable aperture patterns.

Table 8.4 Second lower bound for the equivalent permeability of variable aperture patterns. 
Nomenclature

A

$\boldsymbol{b}$

$b_{\max }$

$b_{c u t}$

$B R E A K$

$C P$

D

F

g

$H_{i j}$

$\vec{j}$

K

$\boldsymbol{K}_{\boldsymbol{k}}$

$\boldsymbol{K}_{\mathbf{l}}$

$K_{\text {a }}$

$\hat{K}_{\text {eq }}$

$K_{\text {aff }}$

l

l。

$l_{1}$

$N$

NBREAK

$N C P$

NSIGC

NSIGR

$P$

p

$p_{c}$

$\vec{q}$

$Q$

$Q_{\max }$

REV

$\vec{r}$

$S_{2}(\vec{r})$

s.e.

SIGC channel width of trapezoidal pattern

$L$

aperture

$L$

maximum aperture in a pattern

$L$

aperture cutoff

$L$

erosion statistic

change point statistic

radius of moving average filter

number of contact area features in a pattern

gravitational acceleration

$L T^{-2}$

moving average filter coefficient

electric current density

Permeability

amperes $L^{-2}$

high permeability value

$L^{2}$

$L^{2}$

low permeability value

$L^{2}$

Equivalent Permeability

Predicted Equivalent Permeability

effective permeability

$L^{2}$

length of pattern

$L$

trapezoid side length

$L$

trapezoid side length

$L$

Length of pattern in pixels

pixels

normalized erosion statistic

normalized change point statistic

normalized column dispersion statistic

normalized row dispersion statistic

pressure

$M L^{-1} T^{-2}$

void fraction

percolation threshold

volumetric flow per unit area

$L T^{-1}$

volumetric flow

$L^{3} T^{-1}$

maximum possible volumetric flow

$L^{3} T^{-1}$

Representative volume scale

pixels

separation vector

pixels

two-point correlation function

standard error

column dispersion statistic 
SIGR row dispersion statistic

i

percolation theory exponent

$\vec{u}$

fluid velocity

$L T^{-1}$

$\checkmark$

Voltage

volts

$X_{i j}$

independent Gaussain assigned to $i j^{\text {th }}$ pixel

correlated Gaussain assigned to $i j^{\text {th }}$ pixel

electrical conductivity

moving average filter parameter

amperes volts $L^{-1}$

$\boldsymbol{\kappa}$

fluid viscosity

pixels $^{-1}$

$\boldsymbol{\mu}$

average

$M L^{-1} T^{-1}$

$<>$ 


\section{INTRODUCTION}

\section{Basic Problem}

Because wastes from nuclear reactors remain dangerously radioactive for over 10,000 years, decision makers hope to find a safe and permanent disposal scheme. One scheme under consideration is to bury the waste in an underground repository.

If the rock surrounding the repository were solid, underground disposal would pose no risks. However, the rock is not solid; there are open spaces or voids in the rock. Thus, nuclear wastes dissolved in groundwater might migrate away from the repository through the voids. If the wastes migrate far enough, they could contaminate underground aquifers, rivers or lakes.

The degree of the risk partly depends on how fluias, under pressure, flow through cracks in the rock. Just like cars travel though a city by means of a network of inter-connected roads, fluids can flow through a network of inter-connected fractures. Fractures exist at many length scales and have complex shapes. This thesis focuses on flow through individual cracks, i.e. single rock fractures, from a statistical perspective. Fluid flow through a complex fracture is predicted in terms of statistics computed from the shape of the fracture.

\section{Fracture Geometry}

To make clear what kind of fractures are studied in this work, imagine a solid piece of rock. Now, suppose that the rock fractures into an upper and lower piece of rock. The void space geometry of this single fracture is the space between the upper and lower pieces of rock.

The simplest kind of fracture is the parallel plate fracture. In the parallel plate fracture, the upper and lower surfaces which define the fracture are parallel to one another. Thus, the distance between the rock surfaces is constant. 
However, realistic fractures are not so simple. In a complex fracture, the distance (aperture) between the upper and lower surfaces varies. In this work, we study fractures defined by upper and lower surfaces which either touch or are separated by a constant distance. To define these fractures, a square region of a reference plane is discretized into square pixels. Each pixel is void or contact area. Hence, fractures consist of two-dimensional patterns of void and contact area pixels. To uniquely define the surfaces in terms of aperture, the upper and lower surfaces are assumed to be mirror reflections of each other about the reference plane.

Even with these simplifications, the patterns are complex because there are many ways to arrange voids and contact areas within a fracture. For instance, Figure 1.1 shows a simulated pattern of voids and contact areas. Chapter 3 describes how patterns are simulated by clipping a correlated Gaussian process defined on a $N$ by $N$ pixel square region. How the arrangement of the voids and contact areas within a pattern determines how a fluid, under pressure, flows across a pattern such as the one shown in Figure 1.1, is the main theme of this thesis.

We wish to determine how the geometry of a fracture controls the flow through the fracture. The way to calculate flow in the fracture is to solve the Navier-Stokes equations (which are Newton's Law for fluids) for the pressure and fluid velocity fields within the fracture. Since the equations are nonlinear, solving them for a complex fracture pattern is impractical even on a computer. Because of this difficulty, flow is modeled using a linear empirical model; local flow is assumed proportional to local pressure gradient times local aperture cubed. Although simpler than the NavierStokes, this flow model still has to be solved on a computer. Chapter 4 describes the flow model and how the method of finite-differences is used to solve for flow numerically. The computed flow through a pattern of voids and contact area pixels is normalized by dividing it by the flow that would had occurred if each pixel had been void. This normalized flow ranges from 0 to 1 and is called the equivalent 

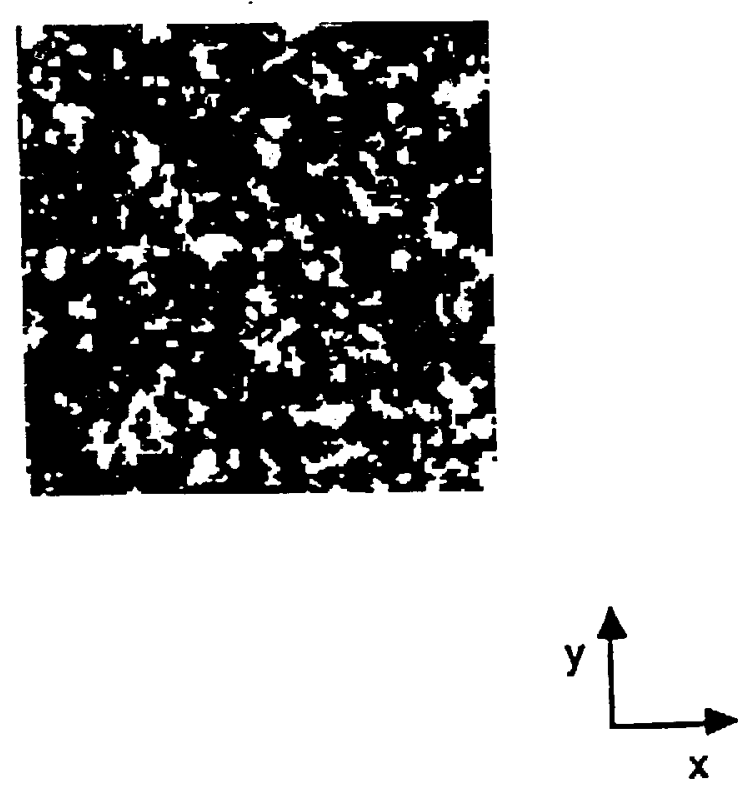

$x B 2093-1052$

Figure 1.1 A simulated pattern of voids(black) and contact areas(white). 
permeability of the pattern. The point of this thesis is to relate the spatial arrangement of the voids and contact areas within a partern to the equivalent permeability of the pattern.

The equivalent permeability of a pattern depends on how well the contact areas block flow. Intuitively, contact area features which are stretched out parallel to the overall flow direction block flow less efficiently than features which are stretched out in the direction perpendicular to the overall flow direction. For instance, in Figure 1.2, for flow in the direction of the arrows, the contact area feature in pattern $\mathrm{A}$ blocks flow more efficiently than the contact area feature in pattern B. However, the shapes of the individual contact area features in a pattern do not completely determine flow. For instance, consider patterns $\mathrm{C}$ and D shown in Figure 1.3. Although both patterns have the exactly the same two contact area features, pattern $\mathrm{C}$ will block flow more efficiently than pattern $D$ if a pressure drop is applied in the direction of the arrows. Hence, for these two patterns, the relative location of the contact area features is important for predicting flow.

In this work, the flow through a specific pattern of voids and contact areas is predicted in terms of spatial statistics computed from the arrangement of voids and contact areas pixels within the pattern. Each spatial statistic measures, in a different way, how well contact area features block flow. The spatial statistics are sensitive to contact area feature shapes and the location of the contact area features relative to the applied pressure drop and each other.

Our philosophy towards the prediction of flow through a pattern of voids and contact areas is empirical. No prior knowledge of how the voids and contact areas are arranged is used to compute the spatial statistics. In contrast, other approaches such as Effective Medium Theory (Kirkpatrick, 1973) or Percolation Theory (Broadbent and Hammersley, 1957) predict flow through iarge patterns in terms of prior knowledge about the way a pattern is simulated. These theories predict flow through 

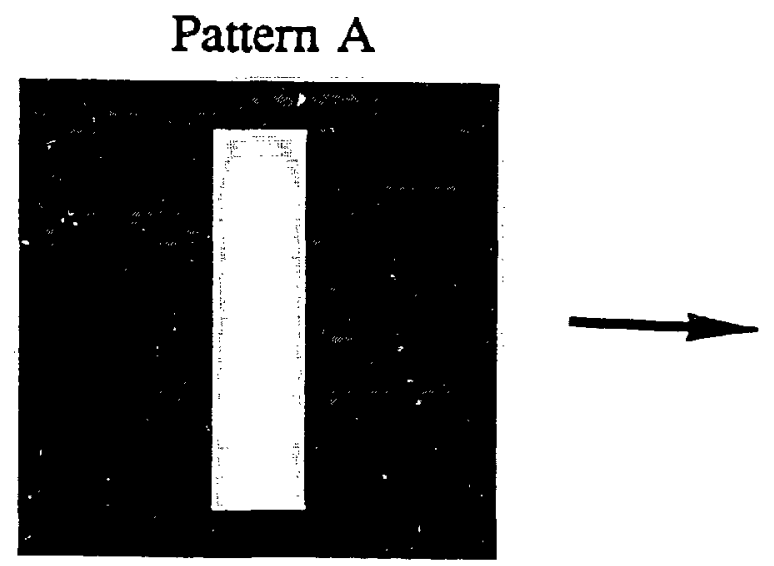

\section{Pattern B}
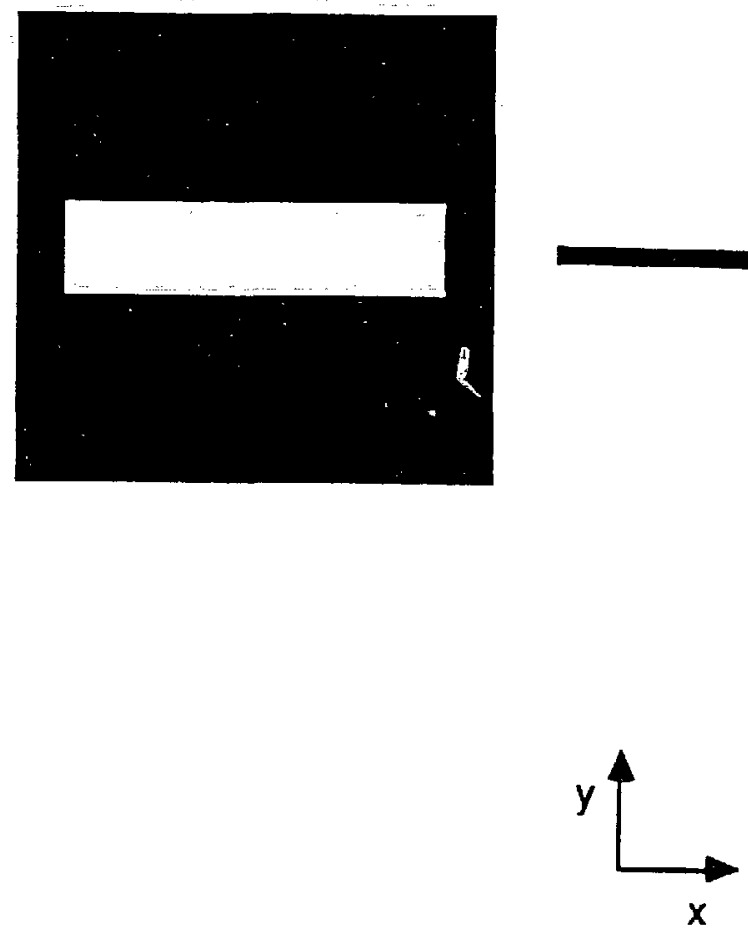

XBL 893-1047

Figure 1.2 For flow in the $x$ direction, the contact area feature (white) in Pattern A blocks flow more efficiently than the contact area feature in Pattern B. 


\section{Pattern C}
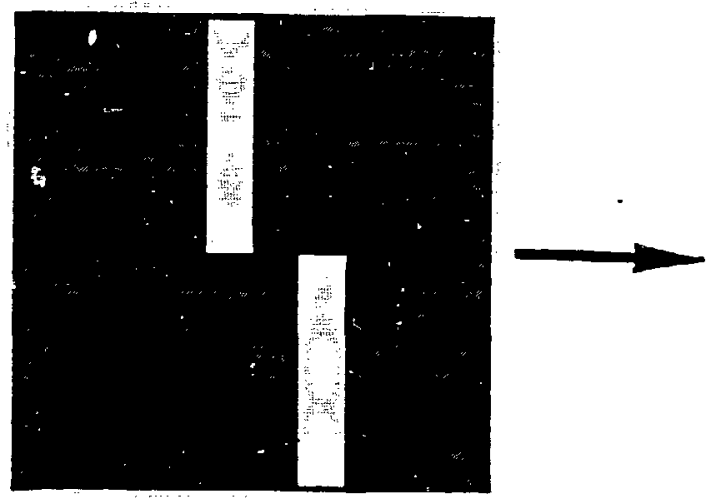

Pattern D
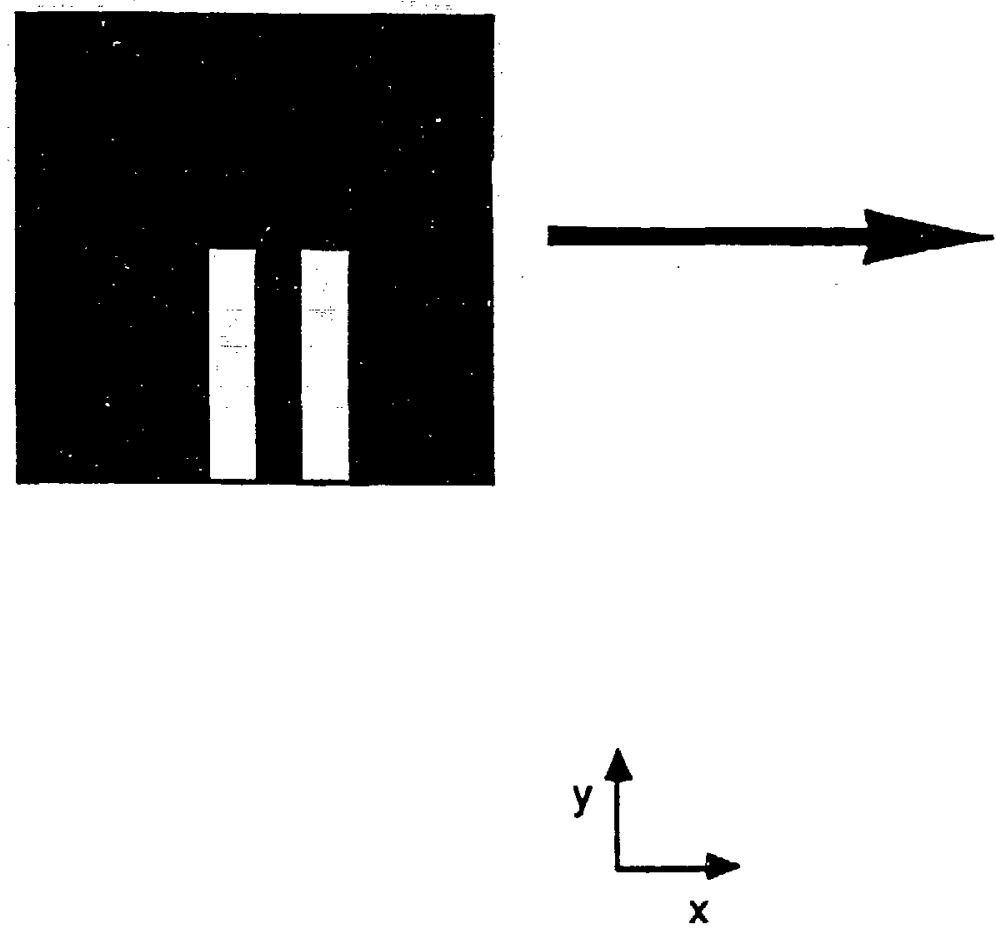

XBL 893-1048

Figure 1.3 Even though both Patterns $C$ and $D$ have contact area features with the same shape, a pressure drop in the $x$ direction leads to less flow through Pattem C than through Pattern D. 
homogeneous patterns where flow is determined by the collective behavior of many distinct barriers but none in particular. The summary statistics of the pattern needed for prediction may be very simple, e.g. the void fraction, because information about how patterns are simulated is built into the prediction formula. That is, the prediction formulas are designed for patterns simulated in very specific ways. Prediction techniques presented here are based on explicit measures of the spatial arrangement.

Since the main focus of the thesis is on how the arrangement of voids and contact areas determines flow, patterns with a fixed fraction of voids $(0.80)$ are studied first. This high fraction was chosen because an observed pattern had a similarly high void fraction. Chapter 5 examines the statistical relationship between each spatial statistic and flow through 10 by 10 pixel and 30 by 30 pixel patterns. Flow is predicted in terms of each spatial statistic alone and then in terms of a linear combination of the spatial statistics.

Since fractures of interest have void fractions different than 0.80 in general, the model is extended to pattems with other void fractions in Chapter 6. Âs a test, the extended model is applied to patterns simulated in different ways. Some of the different patterns were simulated by changing the range of correlation in the Chapter 3 simulation model. Others were simulated using fractal (Mandelbrot, 1983) ideas. Fractal patterns are interesting because they are quite inhomogeneous. In general, the extended model accurately predicted the flow through the patterns. Exceptions are noted.

How well the model works for more complex patterns is an open research question. Even if the model fails to predict overall flow through patterns more complex than considered in this thesis, it may serve as a tool to model flow. For instance, suppose that a large $N$ by $N$ pixel pattern is so complex that it is impractical to solve the equations of flow on the computer. The pattern might be partitioned into 30 by 30 pixel blocks. Using our prediction model, a local equivalent permeability could be 
predicted for each block for pressure drops in the $x$ and $y$ direction. Thus, a block with $30^{2}$ bits of information of information (each pixel is yoid or contact) could be reduced to a block with 2 bits of information. It may then be possible to model the flow through the complex pattern in terms of the predicted equivalent permeabilities of the 30 by 30 pixel blocks. Alternatively, the prediction model may serve as a tool to study the influence of selected contact area features on flow. For instance, all contact area features smaller than a certain size might be treated as voids so as to isolate the effect of the largest features on flow. These applications are not pursued in this work.

\section{Ensemble Variability}

As an extension of the main thrust of the thesis, which is to predict the equivalent permeability of a specific pattern of contact areas and voids, Chapter 7 predicts a lower bound for the standard deviation of the equivalent permeability of an ensemble of simulated patterns from the Chapter 3 model. To define the ensemble, consider an infinite pattern. Now imagine that the infinitely large pattem is broken up into $N$ by $N$ pixel blocks. The ensemble of $N$ by $N$ pixel patterns consists of these infinitely many $N$ by $N$ pixel blocks. For each pattern in the ensemble, equivalent permeability is, in general, different. Hence, the standard deviation of the equivalent permeabilities of the $N$ by $N$ pixel patterns in the ensemble is positive. However, as $N$ approaches infinity, each pattern in the ensemble becomes very homogeneous and the standard deviation of equivalent permeability tends to zero. The standard deviation is interesting because it tells us how large a simulated pattern must be before equivalent permeability becomes very predictable.

We call the scale at which the coefficient of variation (standard deviation divided by expected value) of equivalent permeability falls below a very small level $\varepsilon$ the representative volume (REV) scale. From the lower bound for the coefficient of variation, a lower bound for the REV scale is derived. 
To get the lower bound, the expected equivalent permeability of a $N$ by $N$ pixel pattem in the ensemble is related to its void fraction. To make this relation, an empirical model for flow called the Power Average Model (Journel et al., 1986) is assumed. In terms of the correlogram of the simulated patterns, an expression for the standard deviation of void fraction $\left(\sigma_{p}\right)$ for the ensemble is derived. After expanding the Power Average model expression for equivalent permeability as a Taylor series expansion in void fraction, a lower bound for the standard deviation of equivalent permeability $\left(\sigma_{K_{e q}}\right)$ is given.

For patterns with sufficiently low void fractions, the Power Average Model is not appropriate for relating expected equivalent permeability to void fraction. For such patterns, the more relevant Percolation Theory (Broadbent and Hammersley, 1957) is used to relate the void fraction to the expected equivalent permeability of a pattern from the ensemble. Percolation Theory treats flow through systems where flow is almost completely blocked. A lower bound for the ensemble variability of equivalent permeability is then calculated.

\section{Lower Bound for Flow through Patterns with Variable Apertures}

After developing a model to predict the flow through a pattern of contact areas and voids with constant aperture, the natural question to ask is how can the model be applied to patterns with variable apertures. In Chapter 8, a lower bound for the equivalent permeability of a variable aperture pattern is obtained by first transforming all apertures. In the transformation, all apertures below an adjustable cutoff are set to zero and all apertures above the cutoff are set to the cutoff. Since each transformed aperture is less than or equal to each of original aperture, the flow through the original pattern is greater than or equal to the flow through the transformed pattern. This is because local hydraulic conductivity is proportional to aperture cubed in our flow model. Hence, the model prediction for the equivalent permeability of the transformed pattem provides a lower bound for the equivalent permeability of the 
original pattem. A second lower bound is obtained by setting all the apertures above the cutoff to a intermediate value between the cutoff and the maximum aperture in the pattern. The intermediate value chosen is the cube root of the harmonic average of the cube of apertures above the cutoff. A heuristic argument based on circuit theory rules for finding the maximum resistance of a network of variable resistors motivated this second bound. 


\section{LITERATURE REVIEW}

This Chapter reviews literature relevant to the problem of relating the spatial arrangement of voids and contact areas within a fracture to the flow through the fracture. Because of the similarity between Ohm's Law for electric current flow and the fluid flow model assumed in this work, some solid state physics literature is relevant. The review begins with studies of general upper and lower bound for effective permeability. Models which use specific information about the the spatial arrangement of conducting and non-conducting regions in a pattern are then discussed.

\section{Electrical Current and Fluid Flow Similarity}

The equations assumed to govern fluid flow through fractures have the same form as the equations which govem electric current flow through a medium consisting of conductors and insulators. Fluid flow per unit area $\vec{q}$ corresponds to electric current density $\vec{j}$, permeability $K$ corresponds to electrical conductivity $\sigma$ and pressure $P$ corresponds to voltage $V$. For electrical systems, Ohm's Law is

$$
\vec{j}=-\sigma \nabla V .
$$

For fluid flow, the model assumed in this work is is

$$
\vec{q}=-\frac{K}{\mu} \nabla P .
$$

where $K$ is local permeability. Because flow is proportional to pressure gradient, this model is called a Darcy's Law model. Both models have continuity equations. For the electrica! model, $\nabla \cdot \vec{j}=0$. For the fluid flow model, $\nabla \cdot \vec{q}=0$. This analogy has been pursued by Tsang (1984) who modeled fluid flow through a single rock fracture using an equivalent network model where local hydraulic resistance was proportional to the inverse of aperture cubed. 
Beran (1968)

Using a variational approach, Beran bounded the effective permeability of a porous medium which obeys Eq. 2.2 as follows

$$
\left\langle K^{-1}\right\rangle^{-1} \leq K_{d f f} \leq\langle K\rangle
$$

The lower bound is the harmonic average of local permeabilities and the upper bound is the arithmetic average of local permeabilities. The bounds have a circuit theory analogy; the conductance of a network of resistors is maximized when the resistors are in parallel and minimized when the resistors are in series. According to Eq. 2.3, if a fraction $p$ of the local permeabilities equals $K_{h i}$ and a fraction $1-p$ equals 0 , effective permeability falls somewhere between 0 and $p K_{h i}$. These bounds are broad because they do not use specific information about the spatial arrangement of the voids and contact areas within a pattern. However, there are models which relate flow to contact area for specific kinds arrangement of voids and contact area. Below, we describe such models.

Walsh (1981)

Walsh related the shape of contact area features in a rock fracture to flow for a special case. Walsh considered flow between two parallel plates with barriers between them. The barriers extended from the upper to lower plate, were uniformly spaced and had approximately the same cylindrical shape. To model the flow through the fracture, Walsh made an analogy between the physics of fluid flow around the cylindrical barriers and the physics of heat flow around circular shaped insulators in a conducting sheet. Within the fracture, the pressure field was modeled using the Laplace equation $\left(\nabla^{2} P=0\right)$ and fluid flow was assumed proportional to pressure gradient times conductivity. Using an effective medium approximation, where each barrier is viewed as being immersed in a uniform background conductivity due to all the other barriers, he got 


$$
\frac{Q(c)}{Q(0)}=\frac{1-c}{1+c}
$$

where $c$ is the fraction of contact area and $Q(c)$ is the flow through a fracture with a contact area fraction of $c$. Chen et al. (1989) verified the above formula for contact area fractions ranging from 0 to 0.25 .

Although the above expression may be a good approximation for flow when contact areas are uniformly spaced and have nearly circular cross sectional shapes, the formula is not applicable to more complicated arrangements of voids and contact areas.

Chen et al. (1989)

Chen et al. (1989) extended Walsh's result to the case where instead of circular cross section shapes, barriers had elliptical cross section shapes. Each barrier had the same elliptical shape but was randomly oriented with respect to the overall flow direction. Further, the elliptical barriers were uniformly spaced throughout the fracture. In terms of the aspect ratio $\alpha$ which is the ratio of the minor to major ellipse of each barrier, they got

$$
Q(c)=\frac{1-\beta c}{1+\beta c}
$$

where

$$
\beta=\frac{(1+\alpha)^{2}}{4 \alpha}
$$

They verified Eq. 2.5 for patterns with contact area fractions between 0 and 0.05 . Next, an empirical model for more irregular patterns is reviewed.

Journel et al. (1986)

For complicated patterns of high and low permeability regions, Journel et al. (1986) predicted effective permeability using the Power Average Model. Although intended for sandstone and shale systems, the model is applicable to rock fractures 
because the flow models assumed are the same. Random patterns of high and low permeability regions were simulated using a geostatistical method. In two dimensions, square regions were discretized into square pixels and each pixel got a low or high permeability. In three dimensions, a cubic volume was discretized into cubic pixels and each pixel got a low or high permeability. The expected correlegram (Journel and Huijbregts, 1978) of each simulated patterns was known.

The correlogram, $\rho(\vec{r})$, is a normalized spatial correlation function which communicates how likely it is that two pixels are both in high permeability regions. The correlogram is

$$
\rho(\vec{r})=\frac{S_{2}(\vec{r})-p^{2}}{p(1-p)}
$$

where $S_{2}(\vec{r})$ is the two-point correlation function (Beran, 1968). The two-point correlation function gives the probability that two pixels separated by a vector $\vec{P}$ are both are highly permeable. The fraction of highly permeable regions in the pattern is $p$. The correlogram ranges from +1 to -1 . For instance, if local permeabilities are independent, i.e. there is no correlation, the correlogram is unity at lag zero but equal to zero at all other lags.

They solved for the flow through each simulated pattern using Darcy's Law. Pressure was specified at the inflow and outflow boundaries. The Power Average model for effective permeability is

$$
K_{\text {eff }}{ }^{\prime \prime}=p K_{h i}{ }^{\prime \prime}+(1-p) K_{l o}{ }^{\prime \prime}
$$

where $1-p$ is the fraction of pixels with low permeability $K_{l o}, p$ is the fraction of pixels with high permeability $K_{k i}$ and $w$ is an empirical parameter which depends on the way the high and low permeability regions are simulated.

For three dimensional patterns with theoretical correlogram

$$
\rho(h)=e^{\left(-\frac{3 h}{a}\right)}
$$


where

$$
h=\left[r_{x}{ }^{2}+r_{y}{ }^{2}+15 r_{z}{ }^{2}\right]^{\frac{1}{2}}
$$

Deutsch (1988) found a correlation between $w$ and a weighted average of the theoretical correlogram for flow due to a pressure gradient in the $z$ direction. The best fit value of $w$ was

$$
\hat{w}= \begin{cases}6.0 \rho^{*}-1 & \rho^{*} \leq 0.17 \\ 0.9 \rho^{*}-0.13 & \rho^{*} \geq 0.17\end{cases}
$$

where the weighted average of the theoretical correlogram is

$$
\rho^{*}=\sum_{h_{l=0}}^{2 a} \lambda_{h_{l}} \rho\left(h_{l}\right)
$$

where $h_{l}$ is a lag in the $z$ direction and $a$ is a correlation range parameter. The sum is over pixels; $h_{l}$ is incremented by the length of the pixels. The weights have the form

$$
\lambda_{k_{l}}= \begin{cases}b_{1}-b_{2} h_{l} & \text { for } h_{l} \leq 2 a \\ 0 & \text { for } h_{l} \geq 2 a\end{cases}
$$

The terms $b_{1}$ and $b_{2}$ were chosen so that the sum of the weights is unity. Since this relation is for three dimensional flow rather than two dimensional flow, it is not directly applicable to single fractures. However, a similar statistical relationship between the weighted average of the correlogram and $w$ may exist for two dimensional pattems.

Deutsch related the empirical parameter $w$ to the theoretical, i.e. the expected, correlogram of a simulated pattern rather than to statistics computed from the specific arrangement of high and low permeability regions within the pattern. Hence, Eq. 2.8 predicts the same equivalent permeability for simulated patterns with the same fraction of high permeability regions but different spatial arrangements of high and low permeability regions. In contrast, our model predicts different equivalent permeabilities for patterns with different arrangements of voids and contact areas. Further, our 
prediction model works for a variety of patterns, not just patterns where the theoretical correlogram is known.

A limitation of the Power Average Model is the cue for the next model discussed. If the fraction of non-conducting pixels in a simulated pattern increases beyond a critical fraction, there will be no flow across the pattern. For patterns with void fractions close to this threshold, the Power Average Model does not predict flow accurately. However, Percolation Theory (Broadbent and Hammersley, 1957) does predict flow when the fraction of non-conducting pixels is near this threshold.

\section{Percolation Theory}

To start, consider the independent site percolation formulation of Percolation Theory in two dimensions. In independent site percolation, the plane is discretized into pixels which either conduct flow or block flow. Flow can pass from one conducting pixel to another conducting pixel if they share a common side. All conducting pixels have the same conductance. Pixels are independently assigned as conducting with probability $p$. Percolation Theory predicts that if the fraction of conducting pixels falls below a critical threshold $p_{c}$, flow is completely blocked. As $p \rightarrow p_{c}$ from above, the effective conductivity of the system (one if all pixels conduct flow) is conjectured to take the leading order form

$$
\sigma_{e q} \rightarrow \sigma_{o}\left(p-p_{c}\right)^{t}
$$

where $\sigma_{o}$ is some constant. Kesten (1982) remarks that the value the constant $\sigma_{o}$ takes and the rate at which $\sigma_{\text {eq }}$ converges to to the term on the right and what value t takes are open mathematical issues.

According to conjecture, $t$ is independent of the shape of the pixels, but $p_{c}$ depends on pixel shape. For square pixels, simulation studies show that $p_{c}=0.593$ and $t=1.3$ (Orbach, 1986). For the case where pixel conductances are comelated, conjecture has it that $p_{c}$ shifts but ! stays the same (Levinshtein et al., 1984). For some 
correlation models, the conjecture has been confirmed by numerical studies. For instance, for the Ising Model, Stoll and Domb (1979) showed that $p_{c}$ decreased when neighboring sites were positively correlated and that $p_{c}$ increased when neighboring sites were negatively correlated. This is because as the correlation becomes more negative, the probability that two neighboring pixels are both conducting diminishes. Hence, the more negative the correlation is, the less connected neighboring conducting pixels are. Thus, the critical fraction of conducting pixels necessary to form a connected cluster of conducting pixels which spans the entire pattern increases and $p_{c}$ increases.

Besides site percolation, there is also a bond percolation formulation of Percolation Theory where a regular network of nodes and bonds covers the plane. The bonds either pass flow from node to node or block flow from node to node. The percolation threshold depends on the coordination number of the network, i.e. the number of connecting bonds per node in the network. There is also a continuum percolation formulation where conductors of given shapes, such as a circles, are randomly located in the plane. For instance, Pike and Seager (1974) studied randomly located conducting sticks of varying length and randomly located conducting circles in a plane.

As a caveat, Percolation Theory is not intended for patterns too far from the percolation threshold. For instance, Kirkpatrick (1973) remarks that for bond percolation in two dimensions, Percolation Theory is good for systems where the fractions of conducting bonds is within $20 \%$ of the percolation threshold. Next we review an approach intended to model flow for patterns which are far above the percolation threshold.

\section{Kirkpatrick (1973)}

Kirkpatrick (1973) applied Effective Medium Theory to predict the effective conductivity of a regular network of conducting and non-conducting bonds. The approach worked for networks with conducting bonds about $10 \%$ or more above the 
percolation threshold. In the Effective Medium approximation, each bond is viewed as being immersed in the effective conductivity due to all the other bonds. For the case where each bond is independently assigned as conducing with probability $p$ and non-conducting with probability $1-p$, Kirkpatrick expressed effective conductivity as

$$
\sigma_{e q}= \begin{cases}1-(1-p) \frac{z}{z-2} & \text { for } p \geq \frac{2}{z} \\ 0 & \text { for } p<\frac{2}{z}\end{cases}
$$

The coordination number, i.e. the number of bonds that meet at each node of the network, is z. Eq. 2.15 does not apply to the case where conducting bonds are spatially correlated.

\section{Summary}

The models reviewed used prior kruwledge about how patterns of conducting and non-conducting regions were simulated to relate the effective conductivity of a pattern to simple summary statistics of the pattern. For instance, Walsh's prediction for the normalized flow through a pattern is a function of contact ar $\rightarrow$ fraction only because the prediction is for patterns with circular contact areas that are regularly spaced through the pattern. The Power Average Model prediction of effective permeability is relatively simple because it has an empirical parameter which depends on how the patterns are simulated. Kirkpatrick's Effective Medium theory assumes knowledge of the coordination number of the network and that each bond is independently assigned as conducting or non-conducting. Similarly, Percolation Theory predicts equivalent permeability using prior statistical knowledge of how conducting regions are arranged in a pattern. Furthermore, these theories are intended for systems which are very homogeneous. For inhomogeneous patterns these approaches are not appropriate. In contrast, we predict flow through a pattern in terms of spatial statistics computed from the arrangement of voids and contact areas within the 
specific pattern. Further, we will show that our model predicts flow through a variety of patterns. The patterns the model is tested on have varying degrees of homogeneity and are simulated in different ways. Some are simulated by changing the range of correlation in the simulation model described in the next chapter. Other patterns are simulated using fractal ideas (Mandelbrot, 1983). Although our model predicts the equivalent permeability of a variety of patterns, we do not claim that our model will predict flow for all possible kinds of patterns; for arbitrarily complex patterns, no one model can be expected to predict flow accurately. 


\section{SIMULATION MODEL}

\section{Introduction and Summary}

Patterns of voids and contact areas are simulated so as to have complexity similar to an observed pattern of voids and contact areas. This Chapter describes the experiment which revealed the observed pattern and how patterns are simulated. Based on the analysis of these simulated patterns, a model to predict flow across a pattern in terms of spatial statistics computed from the arrangement of voids and contact areas within the pattern is later developed in Chapter 5 and 6.

\section{Experiment and Observed Pattern}

The observed pattem of voids and contact areas came from a metal injection experiment (technique developed by Pyrak-Nolte, 1987) performed on a cylindrical core sample of quartz monzonite from the Stripa mine in Sweden. The core measured $11.6 \mathrm{~cm}$ in diameter and $18.4 \mathrm{~cm}$ in height. The sample contained a single natural fracture orthogonal to the long axis of the core. Hot molten Wood's metal was injected into the voids of the fracture which was under a $10 \mathrm{MPa}$ stress. This corresponds to the insitu stress at 300 meters below the surface. After the metal solidified, the fracture was taken apart and both the upper and lower fracture surfaces were photographed. Because the metal stuck to either the upper or lower fracture surface, two images were superimposed to get a composite image of the voids and contact areas. To get the images, a Zeiss Image Analysis System digitized television camera images of bright metal on darker rock.

The image consists of a pattern of pixels with side lengths of $0.21 \mathrm{~mm}$. Pixels were classified as void space or contact area depending on whether the pixel's grey level intensity was above or below a chosen threshold. We chose a threshold which gave a digital image with roughly the same shapes and contact area percentage as the visual images of metal on rock. However, we have not studied how to select the best 
threshold in this work. Additional sources of error include non-uniform illumination of the fracture; rotation, tilt and translation of the two fracture surfaces relative to each other before superimposition and classification errors due to void regions having shapes different than square pixels. These errors are difficult to quantify and are not studied in this work. The inner 128 by 128 pixel section of the fracture is shown in Figure 3.1. The pattern has a void fraction of $81.7 \%$.

\section{Simulation}

Simulated Patterns are obtained by clipping a correlated Gaussian process. This simulation procedure is similar to other geostatistical simulation techniques (Journel and Isaaks, 1984). To illustrate the simulation model, the steps taken to simulate a 128 by 128 pixel pattern are detailed below.

First, independent Gaussian random variables are assigned to each pixel in a 128 by 128 pixel pattern. Second, a moving average filter is convolved with the independent Gaussians to get a correlated Gaussian process. The filter weights are radially symmetric and decay exponentially. Thus, the moving average filter assigns the $(i, j)^{\text {th }}$ pixel a new Gaussian random variable which is the weighted average of the original independent Gaussians assigned to pixels within a circular neighborhond of the $(i, j)^{\text {th }}$ pixel. Hence, filtering introduces correlation. Third, pixels with Gaussians above a chosen cutoff are void and the remaining are contact. In other words, the correlated Gaussians are clipped.

In summary,

1. Assign each pixel $(\mathrm{i}, \mathrm{j})$ an independent Gaussian $X_{i j}$. Each Gaussian has mean zero and variance one.

2. Convolve $X$ with a filter $H$ to get a correlated Gaussian process $Y$.

$$
Y_{1}=\sum_{k \rightarrow-D}^{D} \sum_{i=-D}^{D} X_{i \rightarrow k,-1} H_{k l}
$$



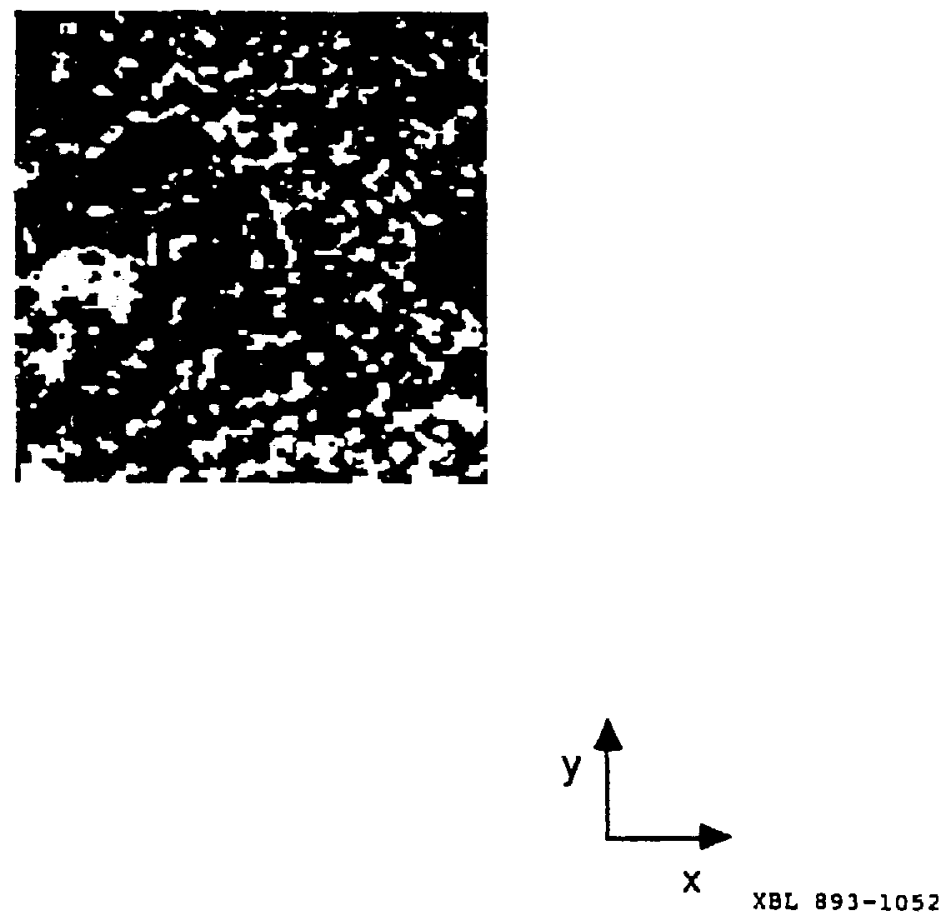

Figure 3.1 Pattern $128 \mathrm{a}$ is an observed 128 by 128 pixel patterm of voids and contact areas within a single fracture. The data is from a metal injection experiment. Voids are black and contact areas are white. Each pixel length is $0.21 \mathrm{~mm} .18 .3 \%$ contact area. 


$$
H_{k}= \begin{cases}C \exp \left(-k \sqrt{k^{2}+l^{2}}\right) & \text { for } \sqrt{k^{2}+l^{2}} \leq D \\ 0 & \text { otherwise }\end{cases}
$$

The normalization factor, $C$, is chosen so that each of the correlated Gaussians, $Y_{i j}$ has mean zero and variance one.

3. Clip

$$
I_{i j}=\left\{\begin{array}{lll}
1 & Y_{i j} \geq Y_{o} & \text { void } \\
0 & Y_{i j}<Y_{0} & \text { coneact }
\end{array}\right.
$$

To simulate a pattern, the following must be specified: $D$ which determines the size of the filter, $\mathrm{x}$ which determines how fast the weights in the filter fall off to zero; the clipping level $Y_{0}$ and the seed for generating the random Gaussians. Rather than quantifying a strategy so as to find optimum values of $x$ and $D, \kappa$ and $D$ were found by the less formal technique of trail and error. The values were adjusted until the simulated and observed pattems had similar correlograms (Journel and Huijbregts, 1978), similar range of contact area feature sizes and similar visual appearances. The values $\mathrm{x}=0.75$ (inverse pixel length) and $D=7$ gave good results. In the trail and error comparison, the value of 0.75 for $\kappa$ gave better results than did values of 1.0 or 0.5. Further, for $\kappa=0.75$, a larger filter $D=10$ gave results similar to the $D=7$ choice. However, for $D=3$ results were worse.

Figure 3.2 shows simulated patterns $128 b, 128 c$ and $128 d$. These should be compared with the observed pattern shown in Figure 3.1. The random number generator was initialized differently for each pattern. For each pattern, the clipping level was adjusted so that the simulated pattern had the same void fraction as the observed patiem.

\section{Correlograms}

The observed and simulated patterns had similar correlograms. The correlogram (Joumel and Huijbregts, 1978) is a normalized spatial autocorrelation function. Let 

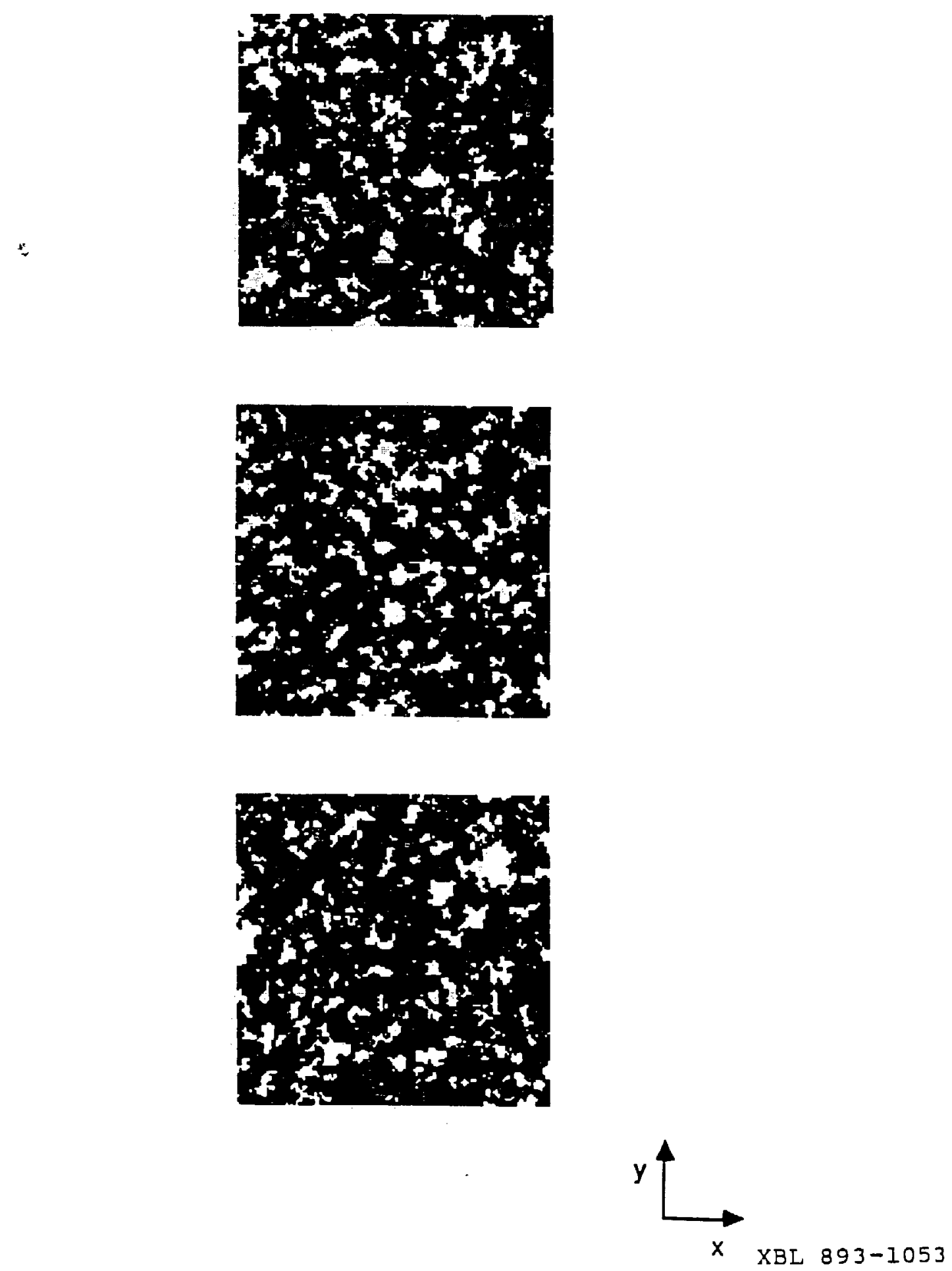

Figure 3.2 Simulated pattems 128b, 128c, 128d. All have $18.3 \%$ contact area. Scale is the same as in Figure 3.1. 
$I(\vec{x})$ be the indicator function for the pixel centered at $\vec{x} . I(\vec{x})$ equals one or zero, depending on whether the pixel centered at $\vec{x}$ lies in a void or contact area. Thus, the average value of $I$ is the average void fraction of the pattern $(p)$. The indicator semi-variogram (Journel and Huijbregts, 1978) at lag $r$ is

$$
x(\vec{r})=\frac{1}{2} E_{x}(I(\vec{x})-I(\vec{x}+\vec{r}))^{2} .
$$

the two point correlation function (Beran, 1968) at $\operatorname{lag} \vec{P}$ is

$$
S_{2}(\vec{r})=E_{x}(I(\vec{x}) I(\vec{x}+\vec{r}))
$$

and the correlogram at $\operatorname{lag} \vec{P}$ is

$$
p(\vec{r})=\frac{S_{2}(\vec{r})-p^{2}}{p(1-p)}
$$

To compute an empirical correlogram for the observed and simulated patterns, pixels in the rows and columns of a pattern are examined. Not all possible pairs are examined; only pixels in the same row or same column are examined. Instead of computing a correlogram which depends on the direction of the lag $\vec{r}$, we compute a correlogram which depends only on the magnitude of $\vec{r}$ which is denoted as $r$. $\hat{S}_{2}(r)$ is the fraction of examined pairs of pixels in the rows and columns, separated by distance $r$, which have two voids. The fraction of pixels in the pairs of pixels examined, $r$ pixels lengths apart, which are void is $\hat{p}(r)$. For finite patterns, $\hat{p}(r)$ is different than the void fraction of the pattern because of sampling error. The empirical correlogram at $\operatorname{lag} r$ is

$$
\rho(r)=\frac{\hat{S}_{2}(r)-\hat{p}(r)^{2}}{\hat{p}(r)(1-\hat{p}(r))}
$$

Figure 3.3 shows that the empirical correlograms of the observed and simulated patterns are close. For lags less than 5 pixel lengths, the match is almost perfect. For lags between 5 and 15 pixel lengths, the correlogram of the observed pattern decays to zero less slowly than do the correlograms of the simulated patterns. There are two 


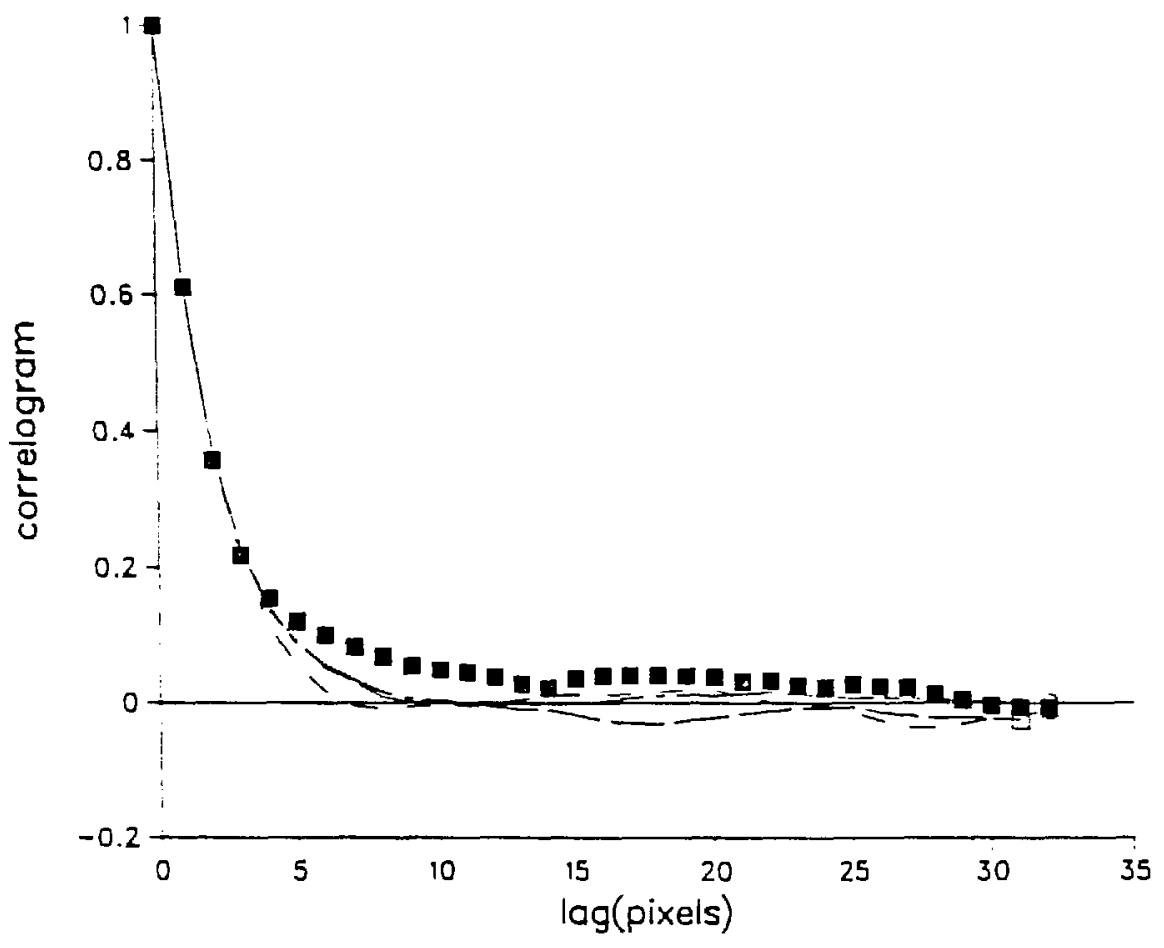

Legend

- $128 \mathrm{a}$

$128 \mathrm{~b}$

$128 \mathrm{e}$ -

$128 \mathrm{~d}$

XBL $893-1054$

Figure 3.3 Correlograms of the observed pattern (128a) and the simulated parterns (128b, 128c, 128d). 
reasons for this discrepancy. First, the observed pattern surely is not exactly like the simulated patterns. For instance, the simulated patterns appear slightly more homogeneous than does the observed pattern; in the observed pattern, the contact area features are not distributed as uniformly as the contact areas in the simulated patterns are. Since the observed pattern is less homogeneous, its expected correlogram should decay to zero less rapidly than the theoretical correlogram of the simulated patterns. Also, the simulated patterns have isotropic correlograms whereas the observed pattern may not. Second, estimated correlograms never exactly equal "true" correlograms because of sampling error. For instance, for lags greater than 15 pixels, the theoretical correlogram of the simulated patterns is exactly zero because the radius of the moving average filter which is used to filter the independent Gaussians is only 7 pixel lengths. However, for lags greater than 15 pixel lengths, the simulated patterns oscillate about zero. The amplitude of the oscillation about zero is a measure of how well determined the correlogram is at these lags.

A model to predict flow is based on smaller, and hence more inhomogeneous, 10 by 10 pixel and 30 by 30 pixel simulated patterns from the model. At these scales, the empirical spatial arrangement of voids and contact of the simulated patterns is neither isotropic nor homogeneous. Further, using a model based on the analysis of flow through these simulated patterns, the flow through the observed pattern will be predicted to within a few percent in Chapter 6 . Therefore, the simulated patterns are complex enough so as to build a model which does a good job of predicting the equivalent permeability of the observed pattern. Thus, fine tuning the simulation model to give patterns which have correlograms that more closely follow the observed would be a lot of effort that may not lead to a better prediction model.

A exponential function was fit to the empirical correlogram by minimizing

$$
\sum_{r=1}^{4}(\log \rho(r)-\lambda r)^{2}
$$


The best fit value was $\hat{\lambda}=0.505$ Figure 3.4 shows that $e^{-0.505 r}$ approximates the correlograms of the 128 by 128 pixel simulated patterns well. Later, this exponential approximation is used for some calculations.

\section{Contact Area Histograms}

Another way to compare simulated and observed patterns, is to compare the histograms of contact area feature sizes. A contact area feature is a distinct cluster of contact area pixels. The size of a feature is the number of contact area pixels that belong to it. Two contact area pixels which share a common side belong to the same feature. But two contact area pixels which are diagonal across from one another do not necessarily belong to the same feature. This definition is the same as the one given by Stauffer (1985) for counting the sizes of clusters. To illustrate these rules, Figure 3.5 shows three patterns of contact areas and voids. Contact areas are white and voids are black. According to the rules, the pattem on the far left has just one contact area feature whereas the other two patterns each have two contact area features. Figure 3.6 shows the contact area feature size histograms and cumulative histograms for the observed and simulated patterns are similar.

To be consistent with the notion that metal can not invade a void which is completely surrounded by contact areas, isolated void space pixels were removed from the 128 by 128 simulated pattems. An isolated void is bordered on all four sides by contact area pixels. The 128 by 128 pixel simulated patterns had a very small percentage of isolated voids-- approximately $0.1 \%$. For all other simulated patterns, isolated voids are left in.

\section{Other simulated patterns}

A model to predict flow in terms of statistics of the spatial arrangement of void and contact areas within a pattern is based on the analysis of smaller 10 by 10 pixel and 30 by 30 pixel patrems simulated by the same model which gave the 128 by 128 pixel patterns. All of the simulated patterns had 0.80 void fractions. Void fraction 


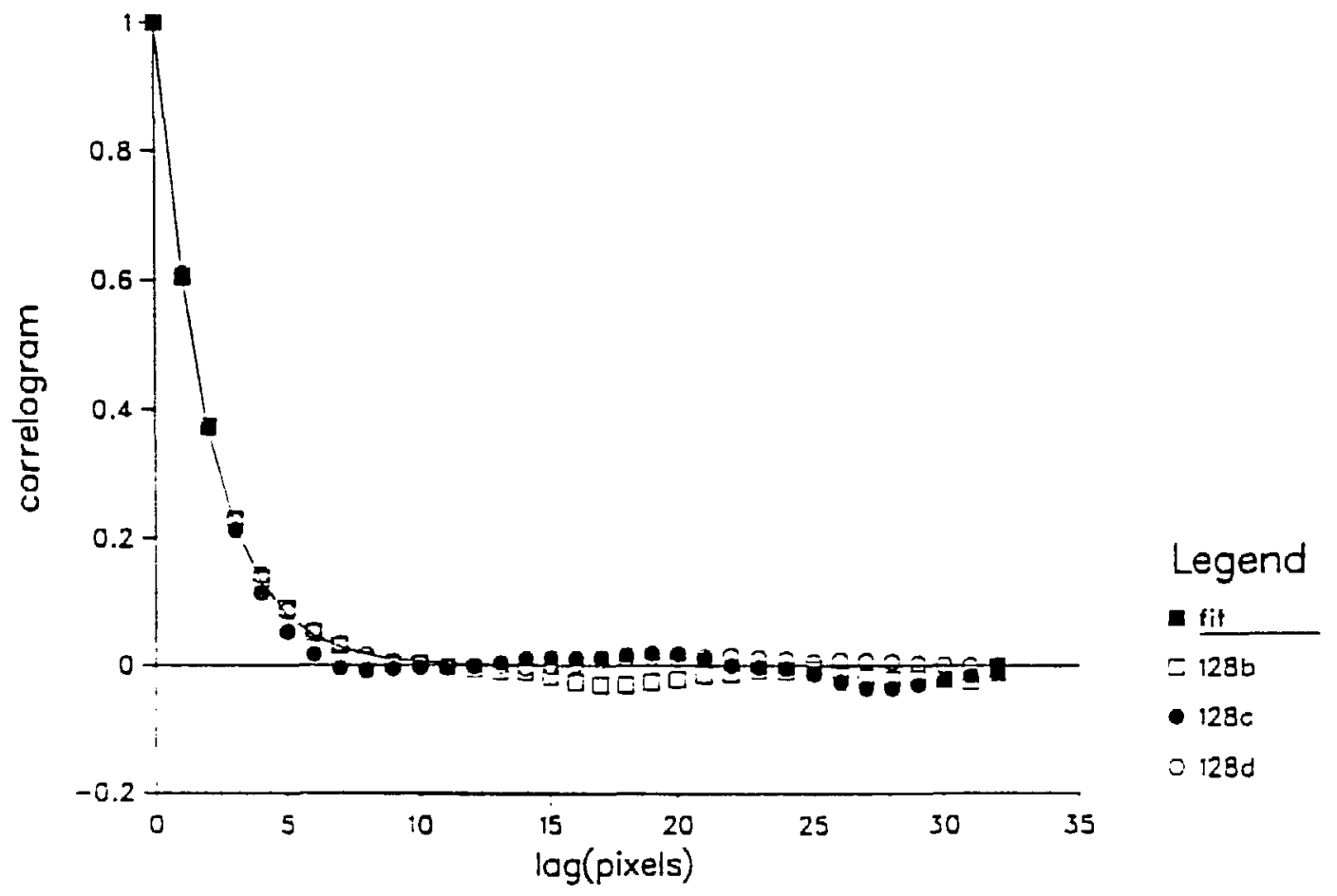

XBL $893-1055$

Figure 3.4 Exponential fit $e^{-\lambda r}$ to correlogram. $\lambda=0.505 . r$ is lag in pixel lengths. 

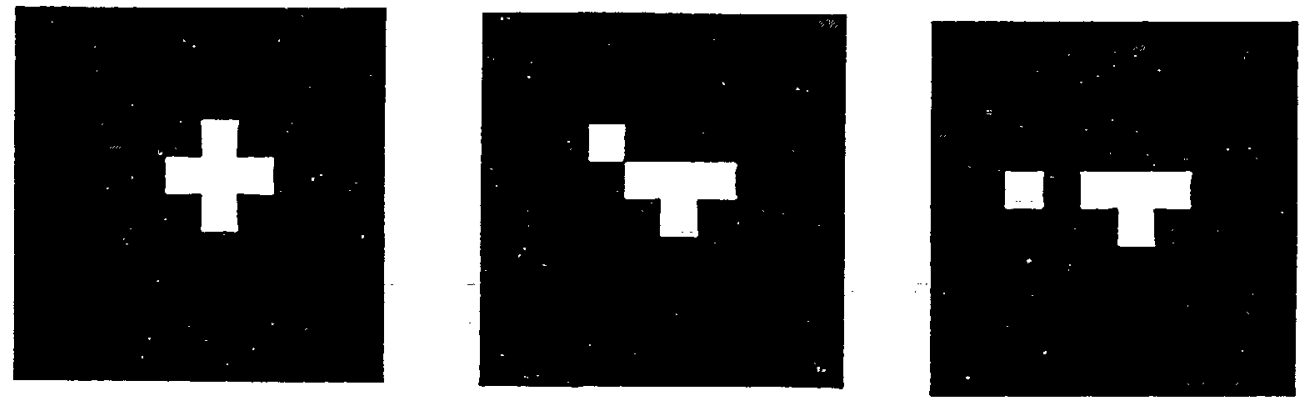

XBL 893-1056

Figure 3.5 Two contact area pixels which share a side are part of the same contact area feature. As an illustration, the pattem on the far left has one contact area feature. Each of the other two patterns have two fearures. 
200 -

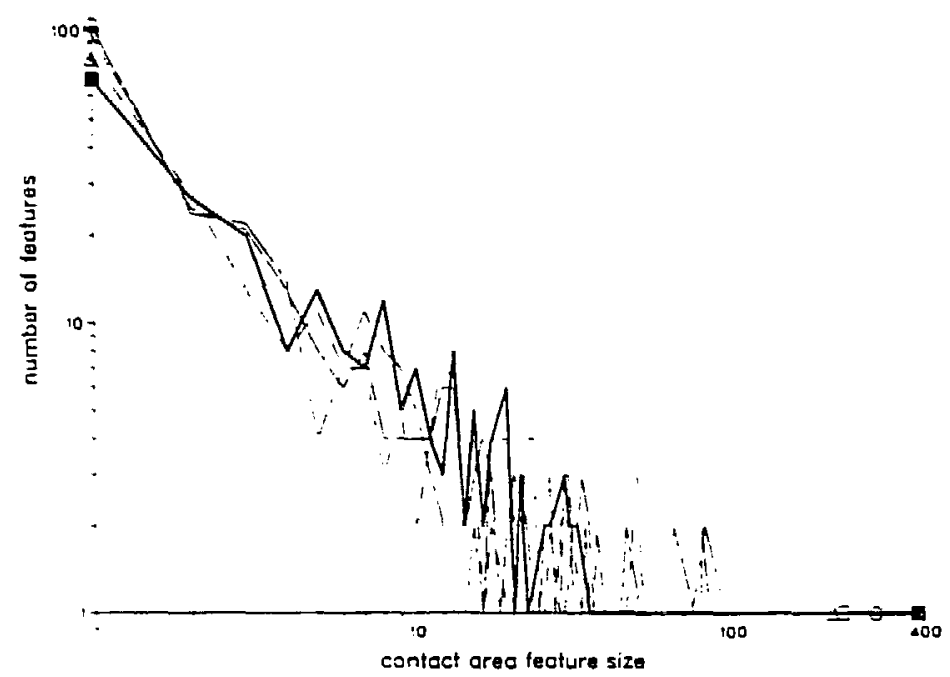

Legend

- 1280

$=\frac{1280}{128 c}$

$2128 c$

$0 \underline{128 c}$.

Cumulative distribution of confact area island sizes

$300-$

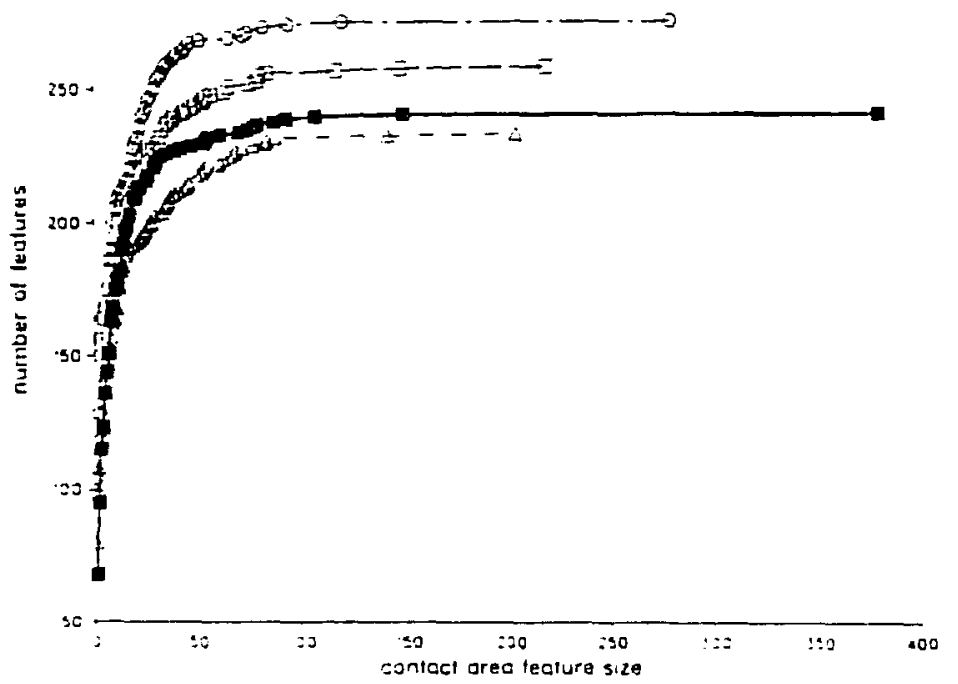

$$
\begin{aligned}
& \text { Legend } \\
& =1280 \\
& =280- \\
& =280- \\
& =28 d
\end{aligned}
$$

Figure 3.6 Histogram and cumulative histogram for contact area feature sizes of observed pattern (128a) and simulated patterns (128b, 128c, 128d). 
was fixed so that the relationship between flow and the spatial arrangements of voids and contact areas could be studied. Later, the prediction model is extended to patterns with other void fractions.

To get the 10 by 10 pixel and 30 by 30 pixel patterns, six 200 by 200 pixel patterns were first simulated. The clipping level $Y_{o}$ was -0.85 for each pattern but the seed used to initialize the computer program which gave the pseudo-random Gaussians was different. The clipping level was chosen to give, on average, patterns with $20 \%$ contact area. From these 200 by 200 pixel patterns, ten non-overlapping 10 by 10 pixel and thirteen non-overlapping 30 by 30 regions which had $20 \%$ contact area were selected. Figure 3.7 shows the 10 by 10 pixel patterns and Figure 3.8 shows the 30 by 30 pixel patterns.

In Chapter 4 , it will be shown that the flow through each of the 10 by 10 pixel and 30 by 30 pixel patterns is highly dependent on the arrangement of voids and contact areas within the fracture pattern. Our goal is to build a model to predict the flow through a pattern in terms of spatial statistics computed from the arrangement of voids and contact areas within a pattern that works for the simulated patterns considered here as well as for more general patterns. The more general patterns could be simulated from other models or be experimental.

\section{Summary}

Patterns of voids and contact areas with complexity similar to an observed pattem of voids and contact areas were simulated. The correlograms and contact area feature sizes histograms of the simulated and observed 128 by 128 pixel patterns were in reasonable agreement. A empirical model to predict the flow through a pattern of voids and contact area pixels is based on the analysis of 10 by 10 and 30 by 30 pixel simulated patterns with $80 \%$ voids. The next chapter describes how flow is computed across each pattern. 

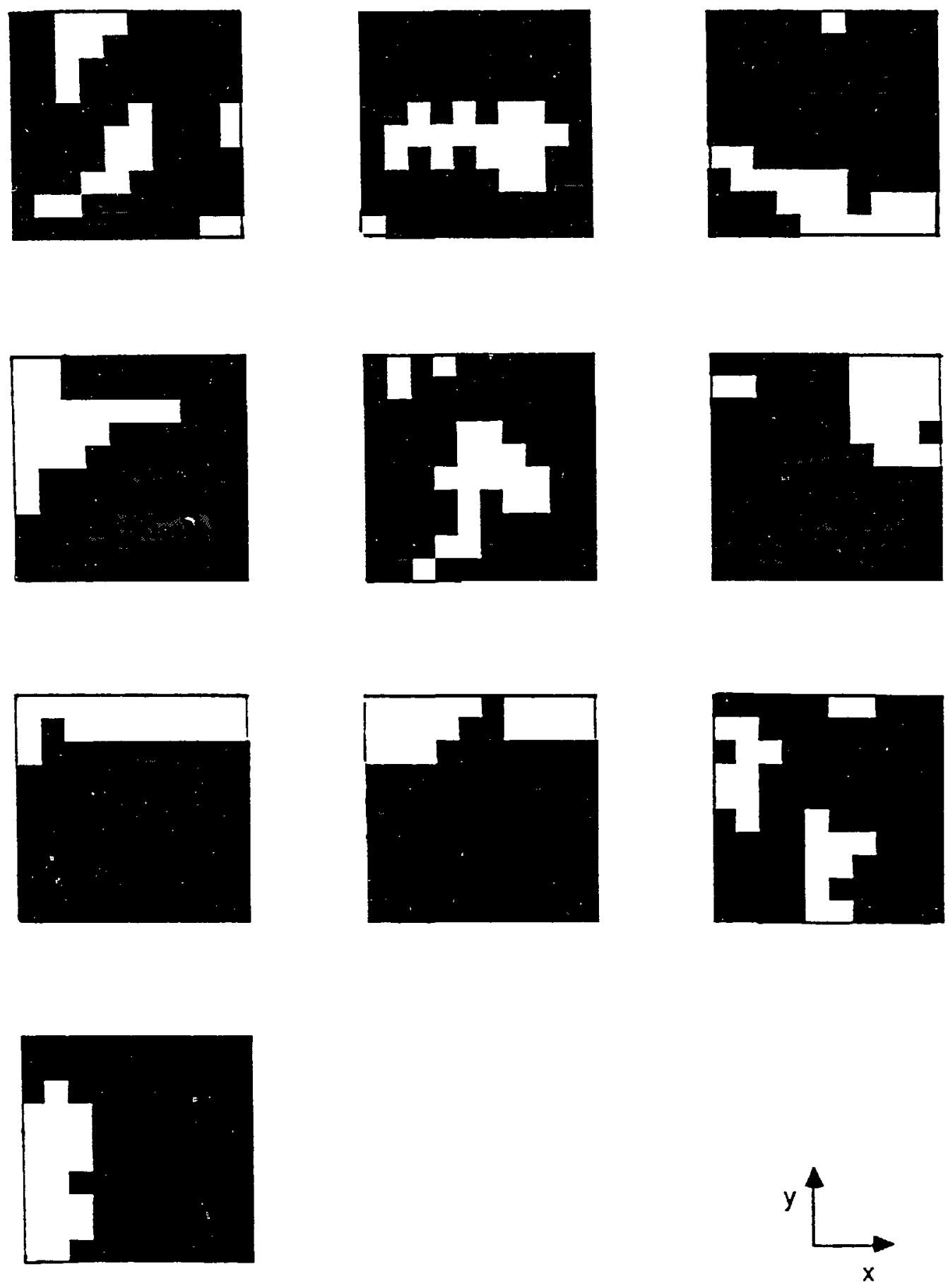

XBL $893-1058$

Figure 3.7 Simulated 10 by 10 pixel pattems with $80 \%$ voids. Patterns result from clipping a correlated Gaussian process. Top row has patterns 10a, 10b and $10 \mathrm{c}$. Second row has $10 \mathrm{~d}, 10 \mathrm{e}$ and $10 \mathrm{f}$. Third row has $10 \mathrm{~g}, 10 \mathrm{~h}$ and $10 \mathrm{i}$. Bottom row has $10 \mathrm{j}$. 

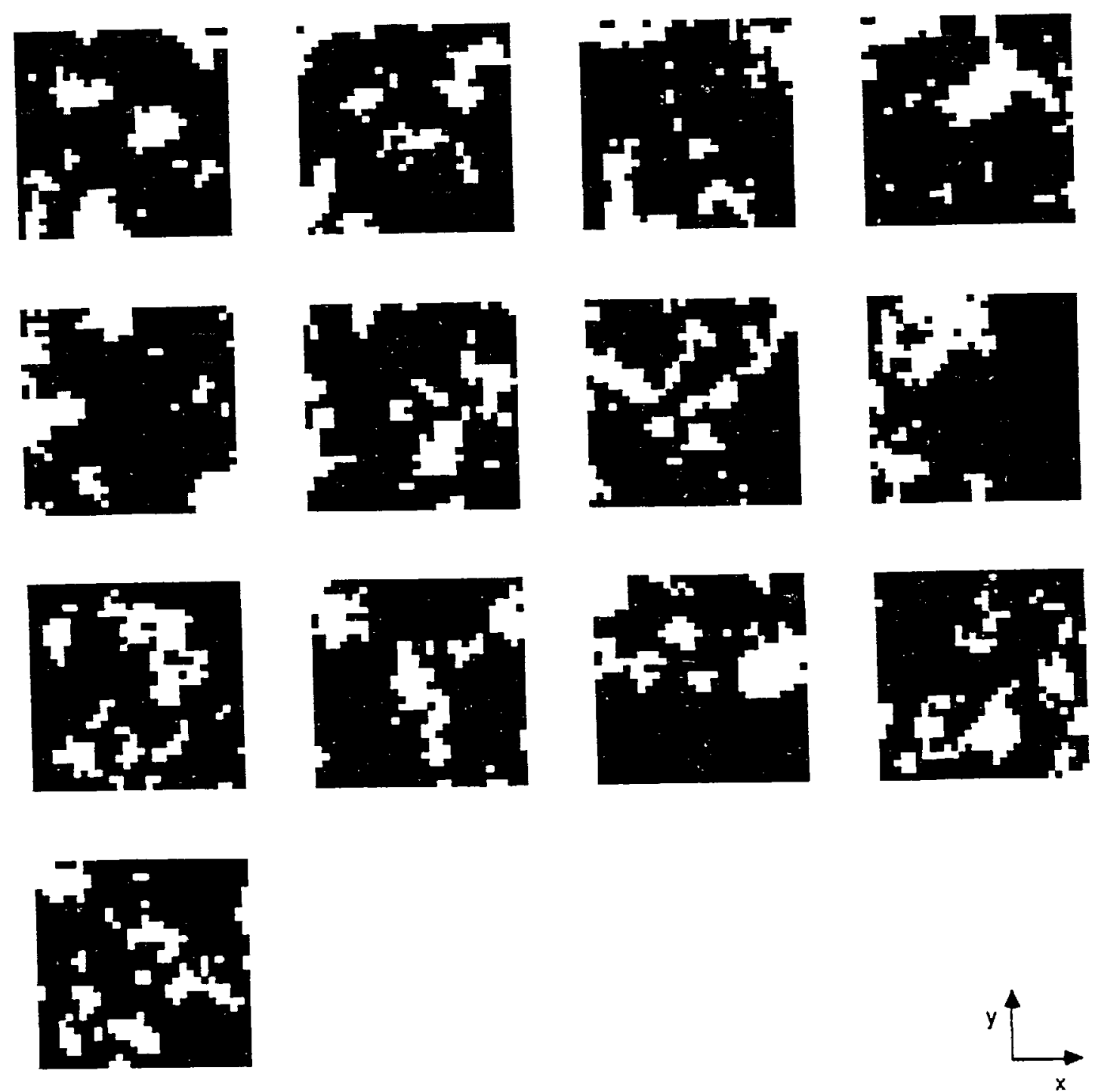

XEL 893-1059

Figure 3.8 Simulated 30 by 30 pixel patterns with $80 \%$ voids. Patterns result from clipping a correlated Gaussian process. Top row has patterns $30 \mathrm{a}, 30 \mathrm{~b}$, $30 \mathrm{c}$, and $30 \mathrm{~d}$. Second row has $30 \mathrm{e}, 30 \mathrm{f}, 30 \mathrm{~g}$ and $30 \mathrm{~h}$. Third row has $30 \mathrm{i}, 30 \mathrm{j}, 30 \mathrm{k}$ and $30 \mathrm{l}$. Bottom row has $30 \mathrm{~m}$. 


\section{FLOW MODEL}

\section{Introduction and Summary}

Since analytical solutions for flow through complicated patterns of void and contact area pixels do not exist, approximate numerical solutions are obtained using the computer. In this thesis, fluid flow is modeled by a local cubic law; local fluid flow is assumed proportional to aperture cubed times local pressure gradient. Approximate solutions for flow are obtained using a finite difference method. The numerical method determined the flow through each of the 10 by 10 pixel and 30 by 30 pixel simulated pattems to a precision of about a percent. Since the variation of equivalent permeability among the simulated patterns is far greater than this numerical error, the numerical method is adequate for the purpose of building a model to predict flow.

\section{Flow model}

For motivation, consider flow between two parallel plates. In cartesian coordinates, suppose the upper plate is at $z=d / 2$, and that the lower plate is at $z=-d / 2$ as in Figure 4.1. Neglecting the effects of gravity, the Navier-Stokes Equations (Schlichting, 1979) for an incompressible fluid are

$$
\nabla \cdot \vec{u}=0
$$

and

$$
\rho_{f} \frac{\partial u}{\partial t}=-\rho_{f}(\vec{u} \cdot \nabla) \vec{u}-\nabla P \cdot+\mu \nabla^{2} \vec{u}
$$

where $\vec{a}$ is fluid velocity, $\rho_{f}$ is fluid density, $\mu$ is fluid viscosity and $t$ is time. If a uniform pressure gradient

$$
\nabla P=\left(\frac{\partial P}{\partial x}, 0,0\right)
$$

forces fluid between the plates, and fluid velocity is assumed to vanish at the plates, the Navier-Stokes equations solution for fluid velocity is 

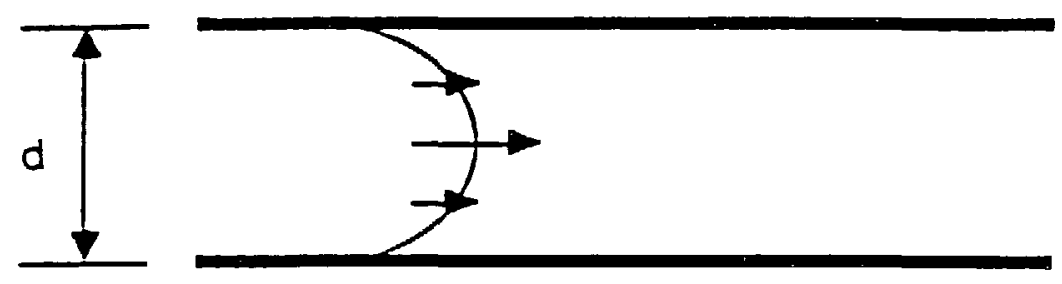

$$
z=d / 2
$$

$z=-d / 2$

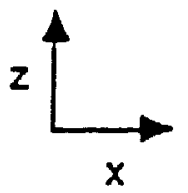

XBL $893-1060$

Figure 4.1 When a uniform pressure gradient in the $x$ direction forces a fluid between parallel plates, the Navier-Stokes equations predict a parabolic fluid velocity profile between the plates and that total flow is proportional to aperture cubed $\left(d^{3}\right)$. 


$$
\overrightarrow{\boldsymbol{u}}=\left(u_{x}, 0,0\right)
$$

where the $x$ component of fluid velocity is

$$
u_{x}=\frac{1}{\mu} \frac{\partial P}{\partial x}\left(\frac{d^{2}}{4}-z^{2}\right)
$$

Schlichting (1979) derives the above result. Note that if the effect of the earth's gravitation field is considered, $P$ in Eqs. 4.2 and 4.3 would be replaced by the difference between the total pressure and the hydrostatic pressure (pressure at rest). For example, if the gravitational field is in the $z$ direction, the pressure minus hydrostatic pressure is $P-\rho_{f} g^{z}$ where $g$ is the acceleration of gravity. However, for gravity in the $z$ direction, Eq. 4.5 would still be valid; the velocity profile is still parabolic and $u_{z}$ is proportional to $x$ component of the pressure gradient. Henceforth, we neglect gravity in our calculations.

The volumetric flow per unit area is in the $x$ direction. The magnitude of the flow rate is

$$
q_{x}=\frac{1}{d} \int_{z=\frac{-d}{2}}^{s=\frac{d}{2}} u_{x} d z=\frac{\partial P}{d x} \frac{d^{2}}{12 \mu}
$$

The total volumetric flow per distance $l$ in the $y$ direction (into the page in Figure 4.1) is

$$
\frac{Q}{l}=\frac{\partial P}{\partial x} \frac{d^{3}}{12 \mu}
$$

Since flow is proportional to aperture cube, this is called the cubic law.

A last remark concerns the permeability of the parallel plates. The empirical Darcy's Law (Beran, 1968) relates permeability $K$ to flow rate per unit area as follows

$$
\vec{q}=-\frac{K}{\mu} \nabla P
$$

Thus, based on Darcy's law, the permeability of the parallel plate fracture is $\frac{d^{2}}{12}$. 


\section{Local Cubic Law Model}

In general, for fractures more complex than parallel plates, there are no simple solutions to the Navier-Stokes equations. To same time and money, rather than solve the Navier-Stokes equations, we assume a approximate flow model called the local cubic law.

In the local cubic law model, fluid velocity between the upper and lower surfaces which define the fracture is assumed parabolic and local volumetric flow per unit area $\vec{q}$, is assumed to be

$$
\vec{q}=\frac{b^{2}}{12 \mu}\left(\frac{\partial P}{\partial x}, \frac{\partial P}{\partial y}, 0\right)
$$

where $b$ is aperture. Conservation of mass gives the following continuity equation

$$
\frac{\partial}{\partial x}\left(\frac{b^{3} \partial P}{\partial x}\right)+\frac{\partial}{\partial y}\left(\frac{b^{3} \partial P}{\partial y}\right)=0 .
$$

Eq. 4.10 is also known as the Reynold's Lubrication Equation. Brown (1987) also assumed this model to solve for flow through simulated fractures.

Note that for the parallel plate where aperture is everywhere constant, the local cubic law model agrees with the Navier-Stokes equations. However, because the local cubic law model ignores drag forces on the fluid accounted for by the NavierStokes equations, the local cubic law overestimates fluid flow for more complex fractures. The magnitude of this error is not studied in this work, but is in Coakley et al. (1989), Muralidhar and Long (1987) and Rothman (1988).

\section{Equivalent Permeability defined}

To actually solve Eqs. 4.9 and 4.10 for a pattem of voids and contact areas, boundary conditions are required. Consider a square pattern of side length $l$ where aperture is defined on the region $0 \leq x \leq l \quad 0 \leq y \leq l$. Consider a left to right pressure drop across the pattern, i.e. a pressure drop in the $x$ direction. For this pressure drop, the boundary conditions are 


$$
\begin{gathered}
P(0, y)=P_{l}+\Delta P \\
P(l, y)=P_{l}
\end{gathered}
$$

and

$$
\frac{\partial P}{\partial y}(x, l)=\frac{\partial P}{\partial y}(x, 0)=0
$$

Figure 4.2 illustrates the boundary conditions.

The flow across the pattern is

$$
Q=\int_{y=0}^{y=1} \frac{b(0, y)^{3}}{12 \mu} \frac{\partial P}{\partial x}(0, y) d y
$$

Rather than report the flow $Q$ which depends on the pressure drop across the pattern and the aperture distribution in the pattern, a normalized flow called equivalent permeability $\left(K_{\text {eq }}\right)$ is reported. Equivalent permeability is

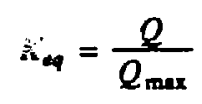

where $Q_{\max }=\Delta p \frac{b_{\max }^{3}}{12 \mu}$ and $b_{\max }$ is the largest aperture in the pattern. $Q_{\max }$ is the flow through the pattem where each aperture equals $b_{\text {max. }} . K_{\text {eq }}$ ranges from 0 to 1 and does not depend on $l, \Delta P$ or $b_{\max }$. Flow can be recovered from equivalent permeability as follows

$$
Q=\Delta P \frac{b_{\max }^{3}}{12 \mu} K_{\infty} .
$$

To illustrate how equivalent permeability depends on void fraction and the arrangement of voids and contact areas, consider the pattern shown in Figure 4.3. Black pixels are voids with constant aperture and white pixel are contact areas. For a pressure drop in the $x$ direction, $K_{\text {eq }}=0.80$. However for a pressure drop in the $y$ direction, $K_{e q}=0$. In general, if a pattem has void fraction $p, K_{e q}$ falls somewhere between 0 and $p$. 


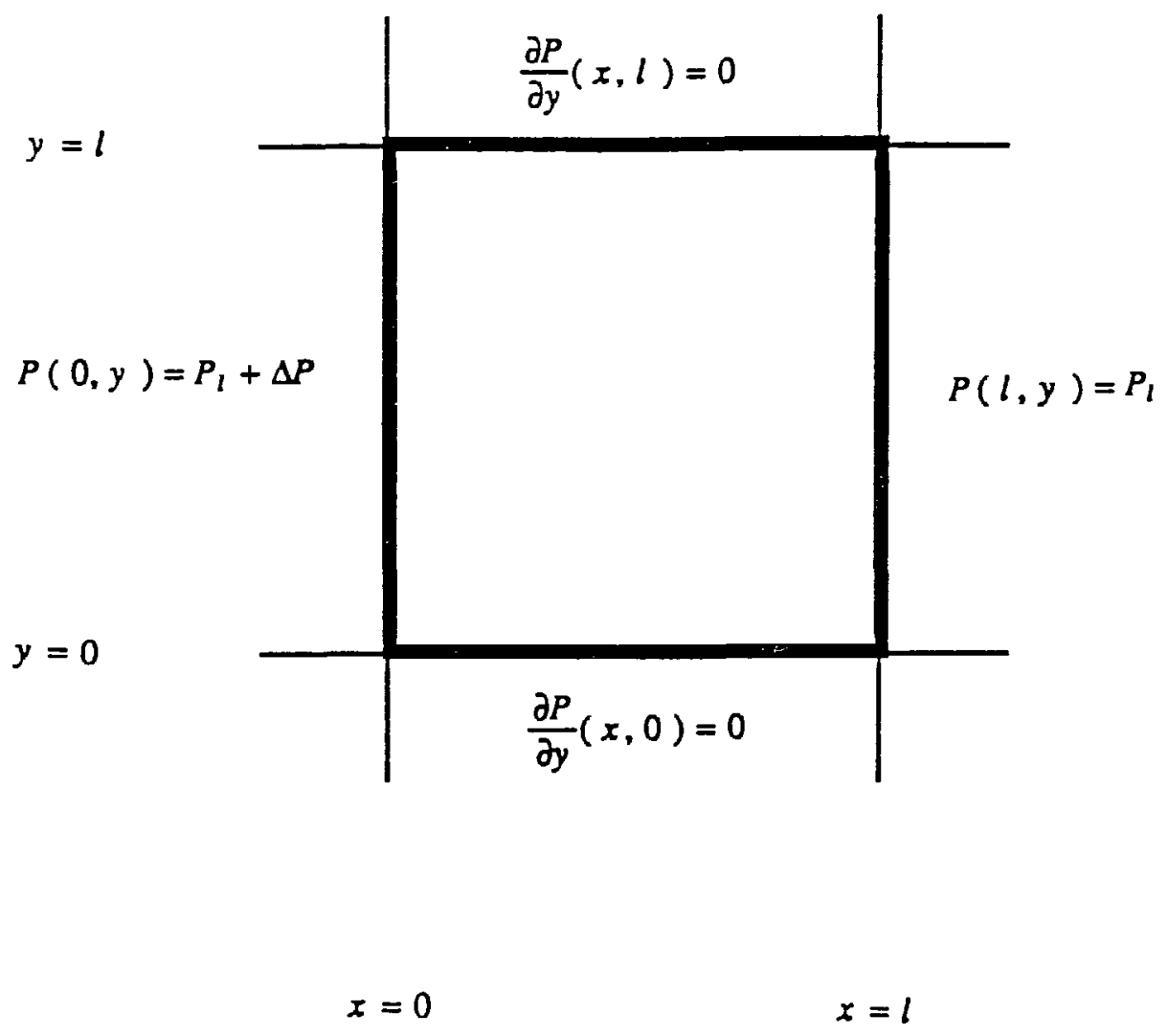

XBL 893-1061

Figure 4.2 Boundary conditions for solving for flow due to a pressure drop in the $x$ direction. 

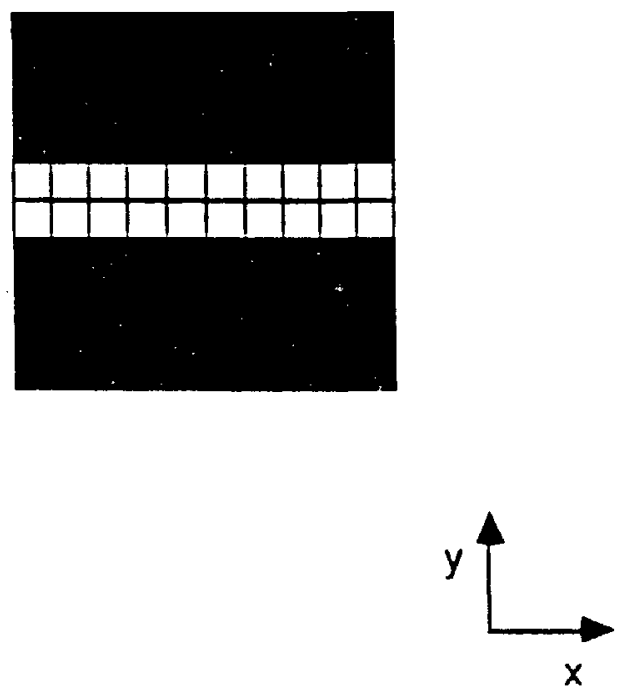

XBL 893-1045

Figure 4.3 A 10 by 10 pixel pattem with $80 \%$ voids. Black pixels are voids with constant aperture. White pixels are contact areas. For flow in the $x$ direction, equivalent permeability is 0.80 . For flow in the $y$ direction, equivalent permeability is 0.0 . 


\section{Numerical Solution}

To get an approximate solution to Eqs. 4.9 and 4.10, a finite difference method was used. The area of the fracture pattern is covered with a finite difference mesh. Neighboring nodes are separated by a distance $\delta$. For instance, for a square fracture pattern with side length $l$ as shown in Figure 4.4, the location of the $(i, j)^{2 h}$ node is $(i \delta, j \delta)$ and the pressure at the $(i, j)^{\text {ek }}$ node is $P(i \delta, j \delta)$. The range of $i$ and $j$ is $\left(0, \frac{l}{\delta}\right)$.

For each pattern of voids and contact areas, flow is solved by the method of finite differences for meshes of various sizes. For larger meshes, the distance between nodes $(\delta)$ is less. To illustrate how a mesh is associated with a pattern, suppose we wish to solve for flow through a 10 by 10 pixel pattern. If a 10 by 10 mesh is used to solve for flow, each pixel would get one node. If a 20 by 20 mesh is used, each pixel gets $2^{2}$ nodes. In general, if a $k$ by $k$ mesh is used, each node is assigned $k^{2}$ pixels. Figure 4.5 shows how many nodes a pixel in a 10 by 10 pattern gets for three meshes-- a 10 by 10 , a 20 by 20 and a 30 by 30 mesh. All the nodes in a particular pixel get assigned the same aperture value. However, nodes that fall in the same pixel are assigned different pressures in general.

At each node in the mesh, a discrete version of the continuity equation relates the pressure at a node to the pressure assigned to its four nearest neighboring nodes. For example, for the center node shown below in Figure 4.6, the continuity equation (Eq. 4.10) gives

$$
\sum_{k=1}^{4}\left(P_{0}-P_{k}\right) b_{k 0}{ }^{3}=0
$$

where

$$
b_{k 0}^{3}=\frac{2 b_{0}^{3} b_{k}{ }^{3}}{b_{0}{ }^{3}+b_{k}{ }^{3}} \text {, }
$$

$P_{i}$ is the pressure at the $i^{\text {th }}$ node, $b_{k}$ is the aperture at the location of the $k^{\text {th }}$ node and $b_{0}$ is aperture at the center node. $b_{k 0}{ }^{3}$ is the harmonic average of the apertures at the 
$(x, y)=(l, l)$

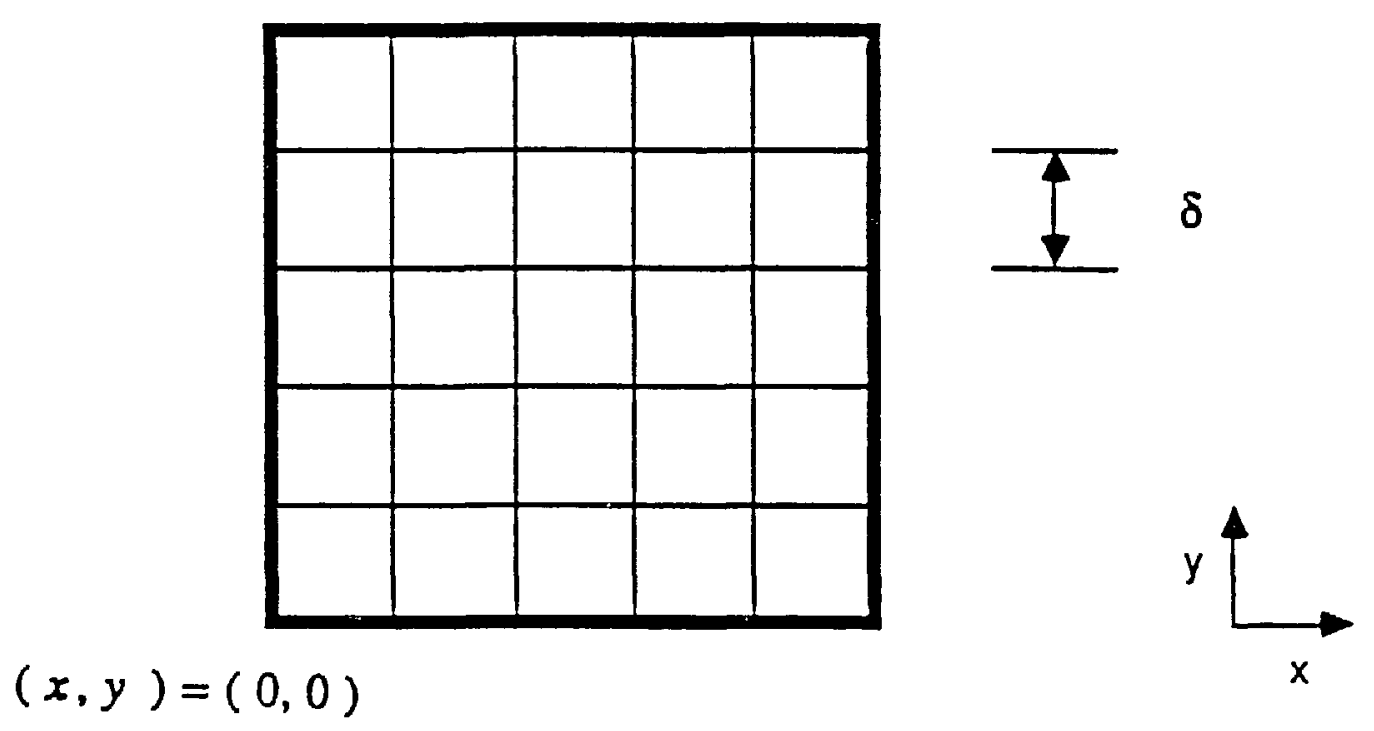

XBI 893-1062

Figure 4.4 In the finite difference solution for flow, pressure is solved for at nodes of a mesh which covers the fracture region. The nodes are where the lines intersect. 

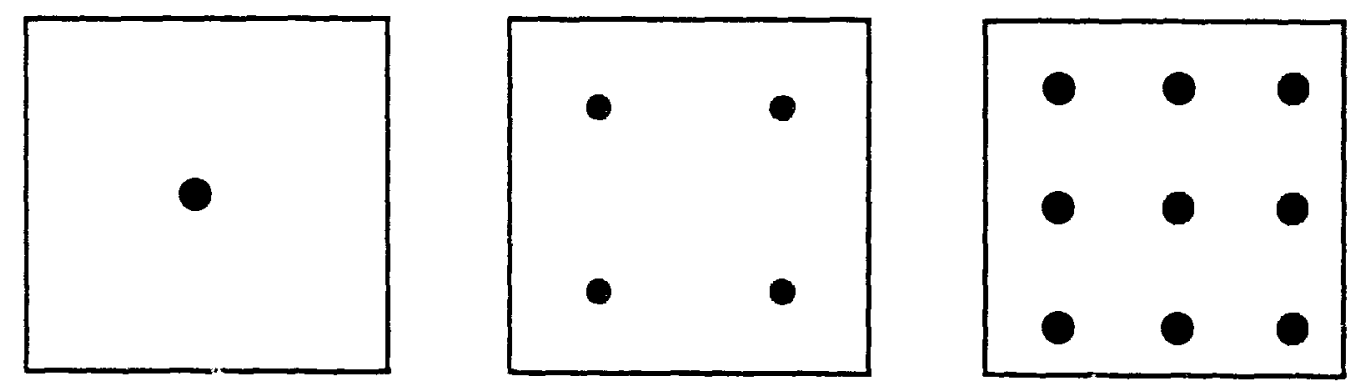

XBL $893-1063$

Figure 4.5 For a 10 by 10 pixel pattern, a finite difference mesh of size 10 by 10 would assign one node to each pixel. A 20 by 20 mesh would assign 4 nodes per pixel. A 30 by 30 mesh would assign 9 nodes per pixel. Above, from left to right, we show how a pixel in a 10 by 10 pixel pattern would be assigned nodes for the three meshes mentioned. 


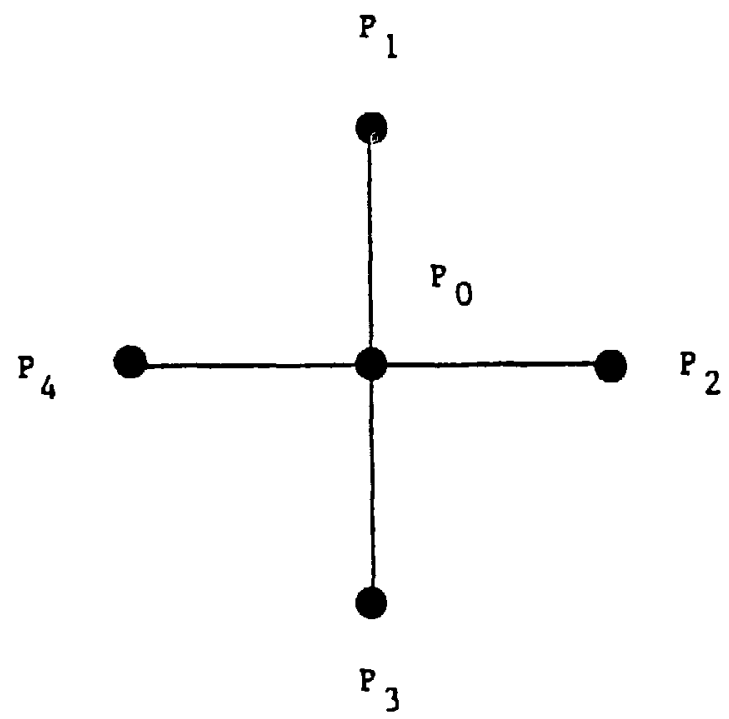

XBL 893-1064

Figure 4.6 Each node in the mesh, except for boundary nodes, has four neighboring nodes. In the finited difference model, the flow between nodes is proportional to the pressure differences between the neighboring nodes times the harmo: :c average of aperture cubed. 
$0^{\text {th }}$ and $k^{\text {th }}$ nodes. This assignment scheme can be viewed as the fluid flow analogy of the electric circuit theory rule that equates the resistance of two resistors in series to the sum of the resistances. In our flow model, hydraulic resistance is proportional to the inverse of aperture cubed. Hence, the arithmetic average of hydraulic resistance is the harmonic average of aperture cubed.

For a pressure drop in the $x$ direction, the discrete version of the boundary conditions (Eq. 4.i1) specifies pressure at the nodes on the inflow boundary $(x=0)$ and the oufflow boundary $(x=l)$ as follows

$$
\begin{gathered}
P(0, j \delta)=P_{l}+\Delta P \\
P(l, j \delta)=P_{l} \quad \text { for } \quad j=0, \frac{l}{\delta} .
\end{gathered}
$$

The boundary condition, $\frac{\partial P}{\partial y}=0$ at the $y=0$ and $y=l$ boundaries, motivates the following boundary conditions on pressure

$$
P(j \delta, l)=P(j \delta, l-\delta) \quad \text { for } j=0, \frac{l}{\delta}
$$

and

$$
P(j \delta, 0)=P(j \delta, \delta) \quad \text { for } j=0, \frac{l}{\delta} \text {. }
$$

To determine pressure, we follow Kirkpatrick (1973), and solve Eq. 4.15 iteratively subject to the above boundary conditions. The iterative method is a Gauss-Seidel method (Strang, 1980). At each step in the iteration, pressure at each node is updated in terms of the pressures at the neighboring nodes during the previous iteration step. For instance, for the center node pictured in Figure 4.6, the iteration step is

$$
P_{0} \rightarrow \frac{\sum_{k=1}^{4} P_{k} b_{k 0}{ }^{3}}{\sum_{k=1}^{4} b_{k 0}{ }^{3}}
$$

To insure convergence, contact areas are assigned very small apertures equal to 
$0.0001 b_{\max }$ rather than zero valued apertures. In all calculations, pressure is updated often enough so that the percentage change in pressure, at each node, between successive iteration steps falls below $0.01 \%$

After solving for pressure, the pressure gradients at the inflow and outflow boundaries are approximated using a three point Lagrangian interpolation formula (Hildebrand, 1974). For instance, the approximation for $\frac{\partial P}{\partial x}(0, j \delta)$ is

$$
\frac{\partial \hat{P}}{\partial x}(0, j \delta)=\frac{1}{2 \delta}[-3 P(0, j \delta)+4 P(\delta, j \delta)-P(2 \delta, j \delta)] .
$$

The flow contributed by this node is approximated as

$$
\hat{Q}_{j}=\delta \frac{b(0, j \delta)^{3}}{12 \mu} \frac{\partial \hat{P}}{\partial x}(0, j \delta) .
$$

Using Simpsons Rule (Hildebrand, 1974), the total flow $Q$ across the inflow boundary is estimated as

$$
\hat{Q}=\frac{1}{3}\left[\hat{Q}_{0}+2 \sum_{j \cos }^{\frac{1}{\delta} 1} \hat{Q}_{j}+4 \sum_{j=0 d d}^{\frac{1}{\delta} 1} \hat{Q}_{j}+\hat{Q}_{\frac{1}{8}}\right]
$$

Simpson's rule approximates the integral of $f(x)$ from $x_{i}$ to $x_{i+2}$ as $\frac{\Delta}{3}\left(f\left(x_{i}\right)+4 f\left(x_{i+1}\right)+f\left(x_{i+2}\right)\right)$ where the distance between $x_{i}$ and $x_{i+1}$ is $\Delta$. The same kind of calculation is repeated for the $x=l$ outflow boundary to get flow out of the pattem. The flows across the inflow and outflow boundaries are averaged to estimate the total flow across the pattern.

\section{Mesh effects}

As the distance between nodes $\delta$ approaches zero (Figure 4.4), estimated equivalent permeability should converge to the real equivalent permeability of the pattem. However, when $\delta$ is not infinitesimally small, computed equivalent permeability depends on mesh size in general. Table 4.1 shows how the computed equivalent permeability of pattem 10e (Figure 3.7 ) depended on mesh size. The applied pressure 
drop was in the $y$ direction. For future reference, in data tables, $(\hat{y})$ denotes that equivalent permeability was computed for a pressure drop in the $y$ direction and $(\hat{x})$ denotes that a flow was computed for a pressure drop in the $x$ direction. In the numerical solution for pressure field, at the coarsest mesh, the initial guess for pressure was the pressure field which dropped off linearly from the inflow to outflow boundary. After the final iteration of Eq. 4.20 for a given mesh, the pressure field was interpolated to get the initial guess for pressure for the next finer mesh.

Table 4.1 Dependence of Equivalent Permeability on mesh size for pattern 10e $(\hat{y})$.

\begin{tabular}{|ccc|}
\hline mesh & iserations & $K_{\text {sq }}$ \\
\hline \hline 10 by 10 & 4000 & 0.663 \\
20 by 20 & 4000 & 0.471 \\
30 by 30 & 4000 & 0.553 \\
40 by 40 & 2000 & 0.572 \\
50 by 50 & 2000 & 0.570 \\
60 by 60 & 2000 & 0.580 \\
70 by 70 & 2000 & 0.577 \\
80 by 80 & 2000 & 0.584 \\
\hline
\end{tabular}

Table 4.1 and Figure 4.7 show that computed equivalent permeability is stable; $K_{e q}$ is the same, within a percent and a half, for all mesher finer than the 40 by 40 mesh. Hence, an 80 by 80 mesh is sufficiently fine to determine the $K_{\iota q}$ of the pattern to within a precision of about a percent. Since the variation in equivalent permeability among the 10 by 10 pixel pattern is much more than this numerical error, equivalent permeability is sufficiently well determined. Table 4.2 lists the equivalent permeability of each of the 10 by 10 pixel simulated patterns computed using a 80 by 80 mesh. For each pattern, equivalent permeability is computed for a pressure drop in the $x$ direction and a pressure drop in the $y$ direction. 
Equivalent Permeabilty versus mesh size

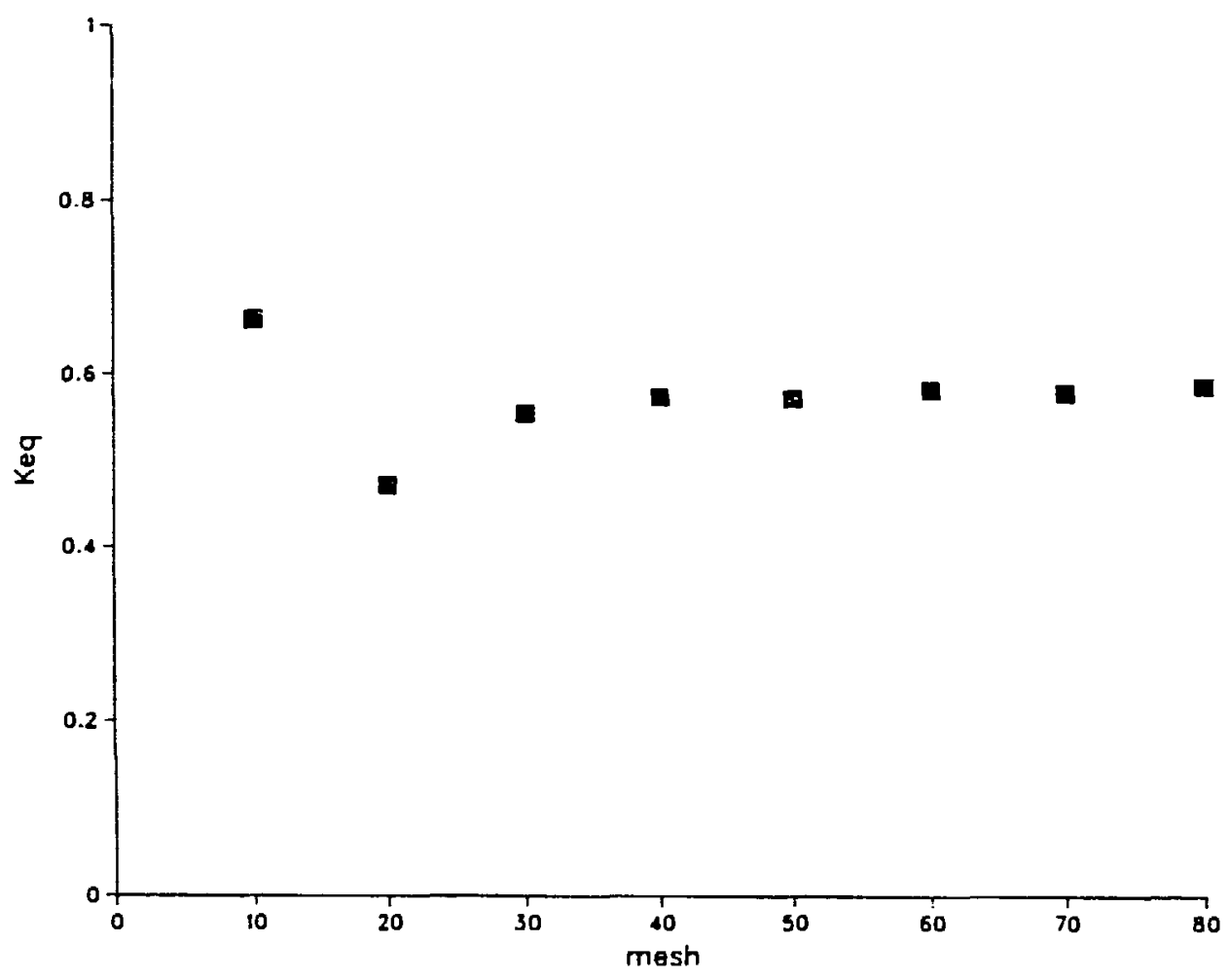

XBL $893-1065$

Figure 4.7 Computed equivalent permeability versus mesh size for pattern $10 \mathrm{e}$. Pressure drop applied in $y$ direction. Mesh size is $\mathrm{k}$ by $\mathrm{k}$. $\mathrm{X}$ axis is $\mathrm{k}$. 
Table 4.2. Equivalent Permeability of the 10 by 10 pixel patterns with $80 \%$ voids.

\begin{tabular}{|ccc|}
\hline Pattern & $K_{\text {aq }}(\hat{y})$ & $K_{\text {eq }}(\dot{x})$ \\
\hline \hline $10 \mathrm{a}$ & 0.564 & 0.457 \\
$10 \mathrm{~b}$ & 0.356 & 0.665 \\
$10 \mathrm{c}$ & 0.000 & 0.670 \\
$10 \mathrm{~d}$ & 0.465 & 0.505 \\
$10 \mathrm{e}$ & 0.584 & 0.324 \\
$10 \mathrm{f}$ & 0.605 & 0.624 \\
$10 \mathrm{~g}$ & 0.000 & 0.776 \\
$10 \mathrm{~h}$ & 0.343 & 0.757 \\
$10 \mathrm{i}$ & 0.622 & 0.414 \\
$10 \mathrm{j}$ & 0.727 & 0.376 \\
\hline
\end{tabular}

For pattern 30b (Figure 3.8), flow was computed for a pressure drop in the $x$ direction. Table 4.3 shows how computed equivalent permeability depended on mesh size.

Table 4.3. Dependence of Equivalent Permeability on mesh size for pattern 30b $(\hat{x})$.

\begin{tabular}{|ccc|}
\hline mesh & itertions & $K_{\text {eq }}$ \\
\hline \hline 30 by 30 & 8000 & 0.474 \\
60 by 60 & 4000 & 0.475 \\
90 by 90 & 4000 & 0.513 \\
120 by 120 & 4000 & 0.514 \\
150 by 150 & 4000 & 0.518 \\
180 by 180 & 4000 & 0.517 \\
210 by 210 & 4000 & 0.519 \\
240 by 240 & 4000 & 0.518 \\
\hline
\end{tabular}

Table 4.3 and Figure 4.8, show that computed $K_{\text {ca }}$ levels off, to within a half percent, for meshes of size 90 by 90 or finer. Since the equivalent permeability of the 30 by 30 patterns varied by much more than this numerical error, equivalent permeability is adequately determined. 
Equivalent Permeability versus mesh size

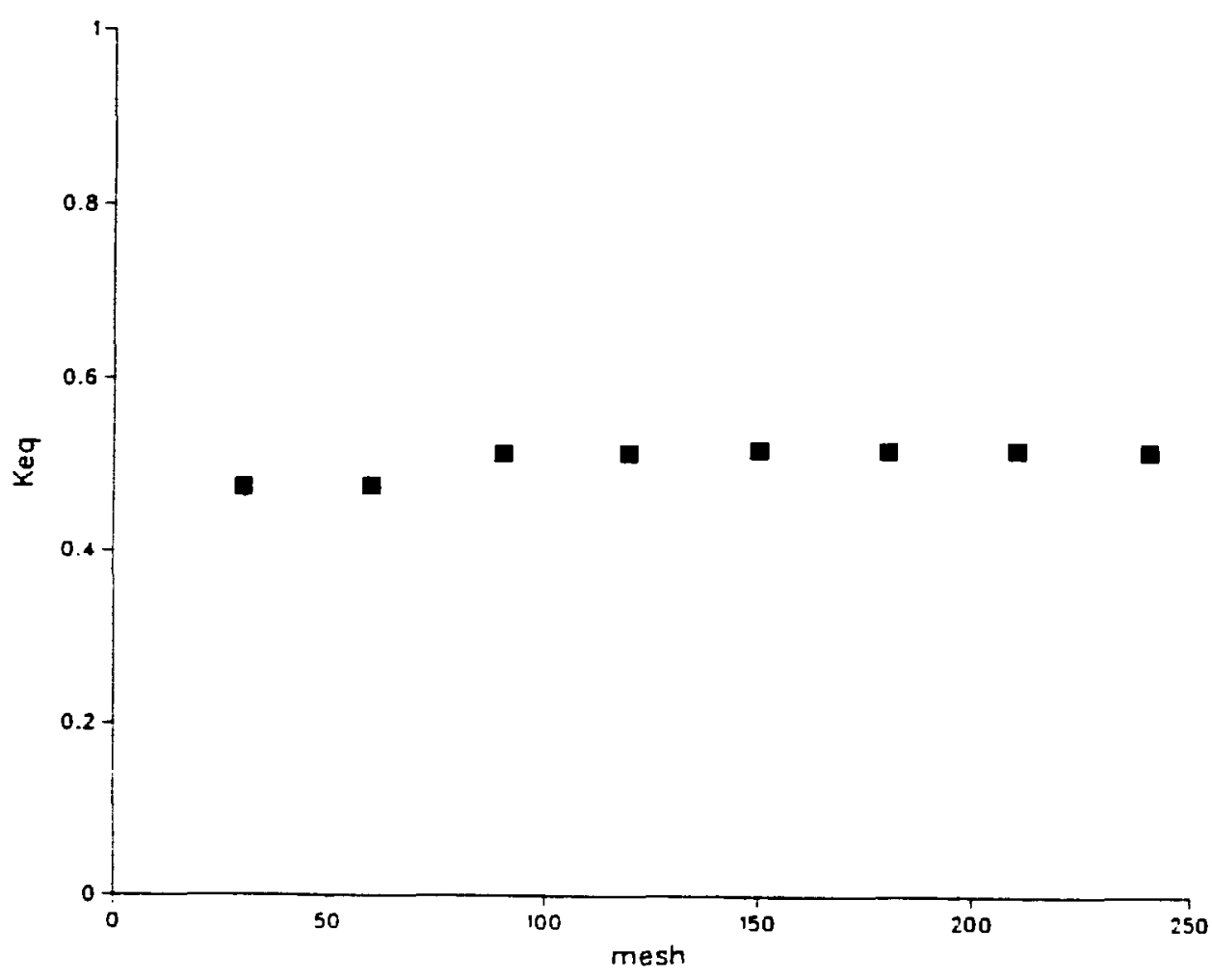

XBL 893-1066

Figure 4.8 Computed equivalent permeability versus mesh size for pattern $30 \mathrm{~b}$. Pressure drop applied in $x$ direction Mesh size is $\mathrm{k}$ by $\mathrm{k}$. $\mathrm{X}$ axis is $\mathrm{k}$. 
Table 4.4 lists the equivalently permeability of each of the 30 by 30 pixel patterns determined using a 240 by 240 mesh. For each pattern, equivalent permeabilities are computed for both pressure drop directions.

Table 4.4 Equivalent Permeability of the 30 by 30 pixel patterns with $80 \%$ voids.

\begin{tabular}{|ccc|}
\hline Paltern & $\boldsymbol{K}_{\mathrm{eq}}(\hat{\mathrm{y}})$ & $\boldsymbol{K}_{\text {eq }}(\hat{x})$ \\
\hline \hline $30 \mathrm{a}$ & 0.580 & 0.567 \\
$30 \mathrm{~b}$ & 0.427 & 0.518 \\
$30 \mathrm{c}$ & 0.510 & 0.507 \\
$30 \mathrm{~d}$ & 0.405 & 0.486 \\
$30 \mathrm{e}$ & 0.606 & 0.535 \\
$30 \mathrm{f}$ & 0.512 & 0.496 \\
$30 \mathrm{~g}$ & 0.247 & 0.486 \\
$30 \mathrm{~h}$ & 0.587 & 0.431 \\
$30 \mathrm{i}$ & 0.553 & 0.422 \\
$30 \mathrm{j}$ & 0.566 & 0.425 \\
$30 \mathrm{k}$ & 0.419 & 0.634 \\
$30 \mathrm{l}$ & 0.439 & 0.525 \\
$30 \mathrm{~m}$ & 0.504 & 0.561 \\
\hline
\end{tabular}

\section{Summary}

A local cubic law model was used to model flow through complex patterns of voids and contact areas. Approximate solutions to the equations of the model were obtained using a finite difference method. The normalized flow through a pattern of voids and contact areas was defined as equivalent permeability. The numerical method determined the equivalent permeability of the 10 by 10 pixel and 30 by 30 pixel patterns to a precision of about a percent. Since equivalent permeability varied from pattern to pattern by much more than a percent, the numerical method is adequate for developing a model to predict equivalent permeability in terms of spatial statistics computed from the arrangement of voids and contact areas within a pattern. 


\section{SPATIAL STATISTICS FOR FLOW}

\section{Introduction}

Now that equivalent permeability has been computed for the simulated patterns of voids and contact areas, we can examine the relationship between spatial statistics computed from the arrangement of voids and contact areas within a pattern and flow. Three criteria described in Section 5.1 guided the selection of the spatial statistics which are examined. The spatial statistics measure how well contact area features block flow and are sensitive to the shape of contact area features as well as the relative location of contact area features in a pattern. Based on the analysis of the 10 by 10 and 30 by 30 pixel simulated patterns with 0.80 void fractions, empirical models to predict equivalent permeability in terms of the individual statistics are presented in Sections 5.2, 5.3 and 5.4. Section 5.5 presents a model to predict equivalent permeability in terms of a linear combination of the spatial statistics. Later, Chapter 6 presents an extended version of the 0.80 void fraction model for patterns with other void fractions. As a caveat, we do not claim that the spatial statistics examined in this thesis are the only reasonable ones to examine. Other choices might lead to empirical prediction models just as good. 


\subsection{MATHEMATICAL PROPERTIES OF SPATIAL STATISTICS}

\section{Introduction and Summary}

Some mathematical criteria motivated by the physics of flow, guided the selection of the spatial statistics computed from the pattern of voids and contact area within a pattern. Below, the criteria are stated.

\section{Directional Dependence}

The flow through a pattern of voids and contact area depends on the direction of the applied pressure drop. For instance, consider patterns A and B which are shown in Figure 5.1.1. Even though the patterns are rotated versions of one another, if the same pressure drop is applied across each pattern in the $x$ direction, flow is greater through pattern B than through pattern A. Hence, like flow, any spatial statistic should depend on the direction of the applied pressure drop.

\section{Statistical Replication Invariance}

The second criteria follows from what is known about continuum limit behavior of homogeneous patterns. Suppose that a infinitely large pattern is simulated using the model presented in Chapter 3. For such a pattern, equivalent permeability depends on the collective behavior of all the contact area features and is exactly predictable in principle. Consistency requires that any spatial statistic converge to a informative asymptotic limit as the size of a simulated pattern from Chapter 3 increases. A informative limit for a spatial statistic can give a predicted equivalent permeability consistent with actual equivalent permeability provided that all model parameters values are properly estimated. Statistics which converge to informative limits are statistically replication invariant because a large homogeneous simulated pattem can be partioned into statistically similar smaller blocks. 

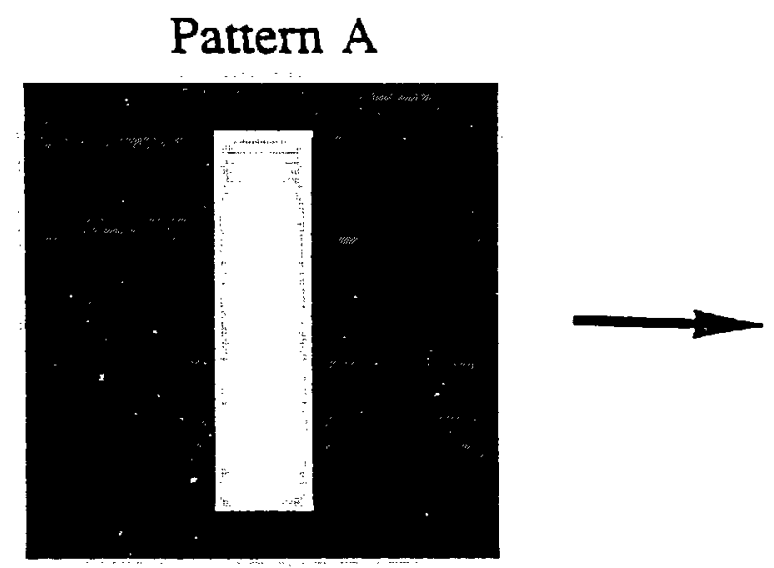

\section{Pattern B}
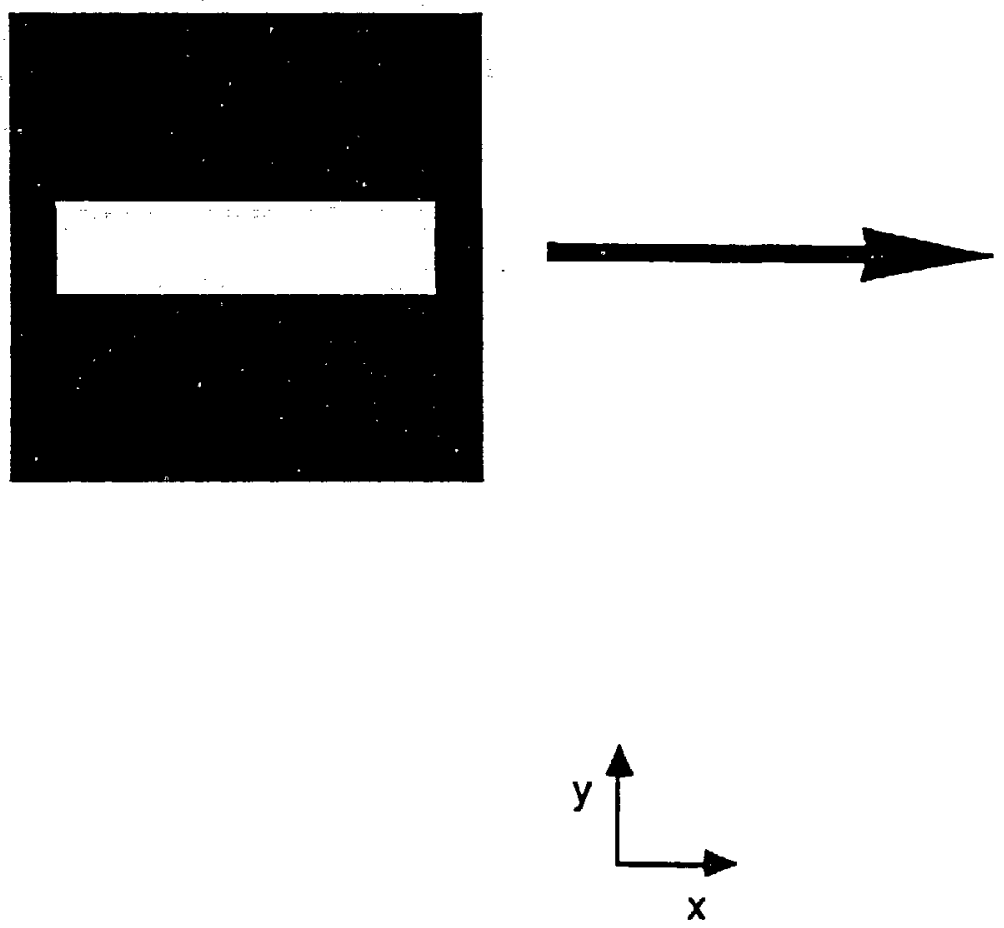

XBL $893-1047$

Figure 5.1.1 Even though patterns $A$ and $B$ are rotated version of one another, for flow in the $x$ direction, the contact area feature in pattern $A$ blocks flow more efficiently than the contact area feature in pattem B. Since flow depends on the direction of the pressure drop, any spatial statist:- computed from a pattern must also depend on the direction of $\because s, \mathrm{ed}$ pressure drop. 


\section{Deterministic replication invariance}

Statistical replication invariance is not demonstrated for all the spatial statistics. For these cases, a property called deterministic replication invariance is demonstrated. Deterministic replication invariance insures that the spatial statistic of a basic pattern agrees with the spatial statistic of any replication of the basic pattern. To illustrate, Figure 5.1 .2 shows a basic pattern and the 2 by 2 and 8 by 8 replications of the basic pattern. To be deterministic replication invariant, the statistics should be the same for all these patterns.

\section{Discretization Invariance}

Another criteria insures that spatial statistics do not arbitrarily depend on the size of the pixels in the pattem. That is, if two discretizations give exactly the same information about the pattem, the spatial statistic must be the same for both discretizations.

To illustrate, suppose a pattern of voids and contact areas is described using pixels which are $k \mathrm{~cm}$ long. Further, suppose the pattern is 10 by 10 pixels in size. Now partition each pixel into four smaller pixels. Each $k \mathrm{~cm}$ long pixel bectres four $\frac{k}{2} \mathrm{~cm}$ long pixels in the new discretization which is now 20 by 20 pixels. Discretization invariance requires that the spatial statistics of the 10 by 10 pixel version and the 20 by 20 pixel version of the pattern agree.

Note, if a finer discretization reveals new details in a pattern, then the two discretizations give different information and a statistic which satisfies the mathematical property of discretization invariance would, in general, depend on pixel size.

\section{Summary}

Based on physical considerations, mathematical criteria that any good spatial statistic should obey were stated. These criteria guided the selection of the spatial spatial statistics which are examined in the next three sections. 

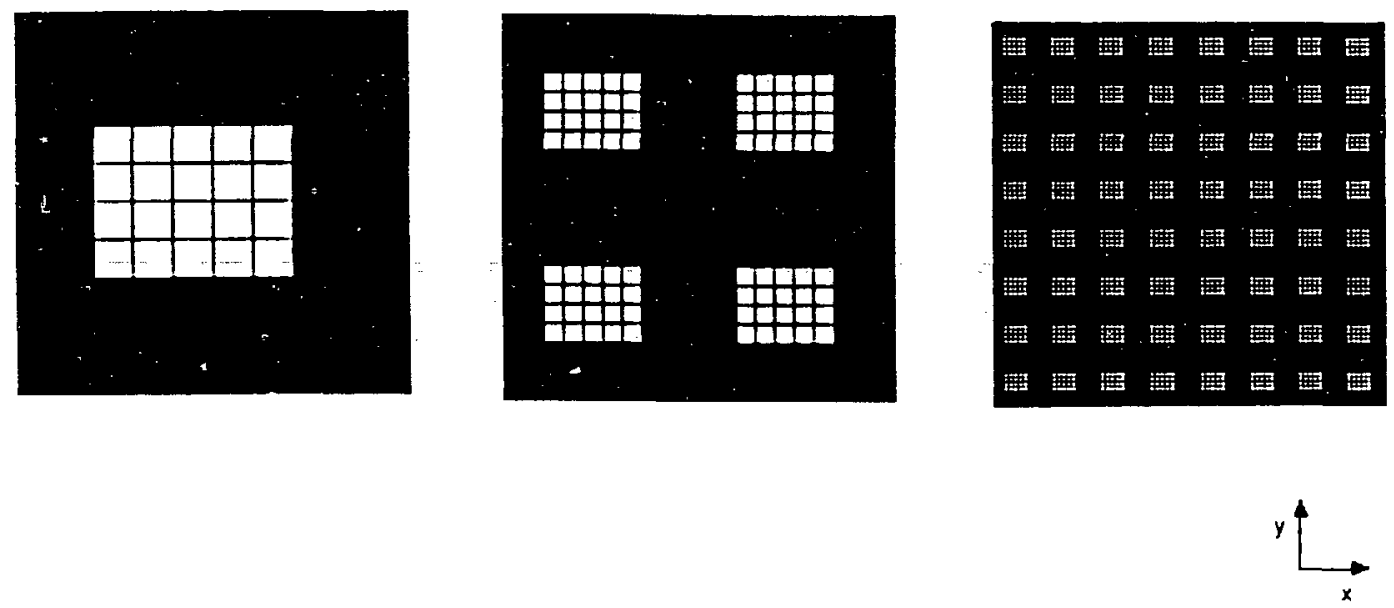

XBI 893-1046

Figure 5.1 .2 A basic pattern ( 10 by 10 pixels), its 2 by 2 replication ( 20 by 20 pixels) and its 8 by 8 replication ( 80 by 80 pixels). Spatial statistics which are determinisitic replication invariant are the same for all three pattems. 


\subsection{THE CHANGE POINT STATISTIC}

\section{Introduction and Summary}

The first spatial statistic considered measures how often adjacent pixels change from void to contact area (or vice versa) in the rows of the pattern which are parallel to the applied pressure drop across the pattern. This change point statistic measures how stretched out contact area features are in the direction of flow. Below, the flow through each of the 10 by 10 and 30 by 30 pixel patterns with 0.80 void fractions is related to the change point statistic.

\section{Motivation}

To illustrate the change point statistic, consider the 10 by 10 pixel pattern shown in Figure 5.2.1. For a pressure drop in the $x$ direction, there are no changes in the rows parallel to the pressure drop and equivalent permeability equals $p$--the void fraction of the pattern. However, for a pressure drop in the $y$ direction, there are two changes per column and equivalent permeability is zero. Hence, a negative correlation is expected between the number of changes in the rows parallel to the applied pressure drop and equivalent permeability.

\section{Statistic defined}

In the rows parallel to the applied pressure drop, $C P$ is defined as the fraction of pairs of pixels which alternate from void to contact or vice versa. For instance, for the 10 by 10 pixel pattern shown in Figure 5.2.1, each column which runs in the $y$ direction has two change points. Since there are nine possible change points in each row of ten pixels, $C P=\frac{2}{9}$ for this pattern.

$C P$ is related to other spatial statistics. Let $I(\vec{x})$ be the indicator function for the void space geometry. $l(\vec{x})$ equals one if $\vec{x}$ lies in a void and zero if $\vec{x}$ lies in a contact area. Hence, $E(I(\vec{x}))=p$. The indicator semi-variogram (Journel and Huijbregts, 

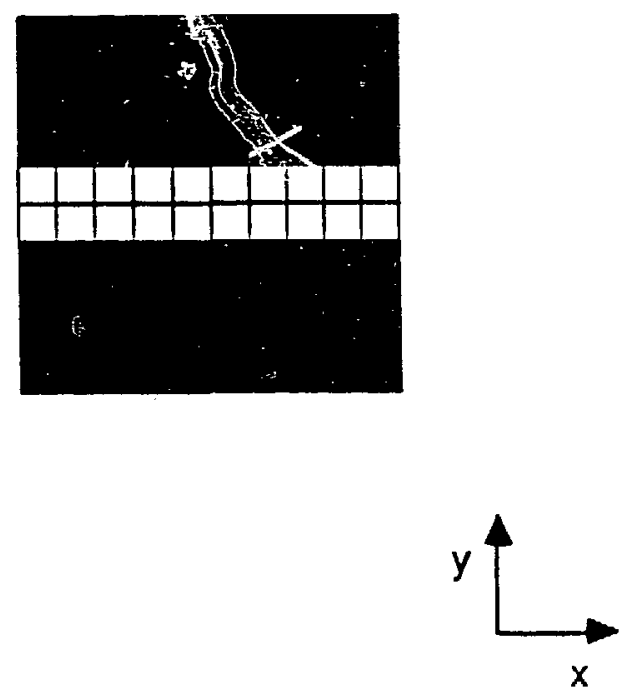

XBL $893-1045$

Figure 5.2.1 In the rows which run in the $x$ direction, there are no alternations between contact areas and voids or vice versa. In the columns which run in the $y$ direction, there are two alternations (change points) per column. 
1978) at lag $\vec{P}$ is

$$
\gamma(\vec{r})=\frac{1}{2} E_{\vec{Y}}(I(\vec{x})-I(\vec{x}+\vec{r}))^{2}
$$

The two-point correlation function (Beran, 1968) at $\operatorname{lag} \vec{r}$ is the probability that two points separated by $\vec{P}$ both lie in voids, i.e.

$$
S_{2}(\vec{r})=E_{\vec{r}}(I(\vec{x}) I(\vec{x}+\vec{r}))
$$

The correlogram (Journel and Huijbregts, 1978) at $\operatorname{lag} \vec{r}$ is

$$
\rho(\vec{r})=\frac{S_{2}(\vec{r})-p^{2}}{p(1-p)}
$$

Thus,

$$
C P=E_{\vec{x}}(I(\vec{x})-I(\vec{x}+\overrightarrow{1}))^{2}=2 \gamma(\overrightarrow{1})
$$

where $\vec{l}$ is a lag of unit pixel length in the direction of the applied pressure drop. Further,

$$
S_{2}(\overrightarrow{\mathrm{l}})=p-\frac{C P}{2},
$$

and

$$
\rho(\overrightarrow{1})=1-\frac{C P}{2 p(1-p)}
$$

Permeability and $S_{2}(\vec{r})$

For a three dimensional porous medium with a isotropic two-point correlation function, Berryman and Blair (1986) bound permeability as follows

$$
K \leq \frac{2}{3} \int_{0}^{\infty} \frac{s_{2}(r)-p^{2}}{(1-p)^{2}} r d r .
$$

Here, $r$ has units of length rather than pixel lengths (which are dimensionless). Because $p-C P=S_{2}(1)$, where 1 denotes a lag of unit pixel length, this bound suggests a negative correlation between equivalent permeability and $C P$. However, the single 
fracture geometry is not isotropic in three dimensions because we can fully describe it by specifying the location of contact areas within a two-dimensional plane. Moreover, a three dimensional porous medium with $100 \%$ voids has infinite permeability whereas a single fracture with no contact area has equivalent permeability equal to one. Therefore, the above bound is merely suggestive that $C P$ carries information about equivalent permeability.

\section{Normalization}

We are not ready to relate $C P$ to flow because it is not discretization invariance. For example, reconsider the 10 by 10 pixel pattern shown in Figure 5.2.1. For a pressure drop in the $y$ direction, $C P=\frac{2}{9}$. But if describe the same pattern with pixels one half as long, i.e. a 20 by 20 pixel description, each of the twenty rows still has only two pairs of alternating pixels, but the total number of pairs of pixels in each row is now 19 . Hence, in the 20 by 20 description, $C P=\frac{2}{19}$. In general, if the pattern is described by $N$ by $N$ pixels, $C P=\frac{2}{N-1}$.

To get a discretization invariant statistic, the obvious thing to do is to multiply $C P$ by $N-1$ where $N$ is side length of the pattern in pixel units. However, $C P(N-1)$ is not deterministic replication invariant. To see why, reconsider the patterns shown in Figure 5.1.2. Figure 5.1.2 shows a basic 10 by 10 pixel pattern and its 2 by 2 replication ( 20 by 20 pixels) and 8 by 8 replication ( 80 by 80 pixels). The basic 10 by 10 pixel pattern has a rectangular contact area of dimension four by five pixel lengths. Hence, for a pressure drop in the $x$ direction, four rows have two change points each. Hence, $C P=\frac{8}{90}$ and $C P(N-1)=\frac{8}{10}$. By a similar argument, $C P(N-1)$ equals $\frac{16}{10}$ for the 2 by 2 replication and $C P(N-1)$ equals $\frac{64}{10}$ for the 8 by 8 replication. In general, $C P(N-1)$ equals $\frac{8 k}{10}$ for the $k$ by $k$ replication of the basic pattern. Because of the $k$ dependence, $C P(N-1)$ is not deterministic replication invariant. 
To get a deterministic replication invariant statistic, $C P(N-1)$ must be divided by something proportional to $k$. Observe that the number of contact area features $F$ in the family of patterns considered in Figure 5.1.2 is proportional to $k^{2}$. Hence, a deterministic replication invariant statistic for the pattems under consideration is

$$
N C P=\frac{C P(N-1)}{\sqrt{F}} .
$$

Besides satisfying deterministic replication invariance, $N C P$ also is statistical replication invariant for simulated pattems from Chapter 3. That is, NCP converges to a non trivial asymptotic limit because $C P$ converges to $2 x(\overrightarrow{1})$ and $\frac{(N-1)}{\sqrt{F}}$ converges to some positive constant as $N$ increases because the range of correlation in the model is finite.

\section{A Geometric Interpretation}

For the 10 by 10 pixel pattern shown in Figure 5.2.1, for a pressure drop in the $y$ direction, $C P$ is $\frac{2}{9}, N-1$ is 9 , and therefore $N C P$ is 2 . For this particular pattern, the contact area feature is 10 pixels long in the direction normal to the applied pressure drop. Thus, for this pattern, NCP is the ratio of the projected perimeter of the contact area feature onto the inflow and outflow boundaries and the length of the pattern. In other words, NCP measures how stretched out the contact area feature is in direction normal to flow. For patterns with many features, NCP is interpreted as a measure of how stretched out, on average, the features in the pattern are. Next, the statistical relationship between flow and $N C P$ is examined for the simulated patterns.

\section{Simulated Patterns}

Table 5.2.1 and 5.2.2 list $C P, F, N C P$ and equivalent permeability for the 10 by 10 pixel and 30 by 30 pixel patterns with 0.80 void fractions. Again, $(\hat{y})$ denotes equivalent permeability for a pressure drop in the $y$ direction and $(\hat{x})$ denotes equivalent permeability for a pressure drop in the $x$ direction. 
Table 5.2.1. Change point statistics and actual and predicted equivalent permeability for the 10 by 10 pixel patterns with $80 \%$ voids.

\begin{tabular}{|c|c|c|c|c|c|}
\hline Pattern & $\overline{C P}$ & $F$ & $N C P$ & $K_{2 q}$ & $\hat{K}_{\infty}$ \\
\hline $10 \mathrm{a}(\hat{y})$ & 0.189 & 5 & 0.760 & 0.564 & 0.546 \\
\hline $10 \mathrm{a}(\hat{x})$ & 0.233 & 5 & 0.939 & 0.457 & 0.486 \\
\hline $10 \mathrm{~b}(\hat{y})$ & 0.189 & 2 & 1.202 & 0.356 & 0.398 \\
\hline $10 \mathrm{~b}(\hat{x})$ & 0.189 & 2 & 1.202 & 0.665 & 0.398 \\
\hline $10 c(\hat{y})$ & 0.167 & 2 & 1.061 & 0.000 & \\
\hline $10 c(\hat{x})$ & 0.100 & 2 & 0.636 & 0.670 & 0.587 \\
\hline $10 \mathrm{~d}(\hat{y})$ & 0.133 & 1 & 1.200 & 0.465 & 0.399 \\
\hline $10 \mathrm{~d}(\bar{x})$ & 0.078 & 1 & 0.700 & 0.504 & 0.566 \\
\hline $10 e(\hat{y})$ & 0.167 & 4 & 0.750 & 0.584 & 0.550 \\
\hline $10 \mathrm{e}(\hat{x})$ & 0.244 & 4 & 1.100 & 0.324 & 0.433 \\
\hline $10 f(\hat{y})$ & 0.111 & 2 & 0.707 & 0.605 & 0.564 \\
\hline $10 f(\hat{x})$ & 0.078 & 2 & 0.495 & 0.624 & 0.635 \\
\hline $10 \mathrm{~g}(\hat{y})$ & 0.111 & 1 & 1.000 & 0.000 & \\
\hline $10 \mathrm{~g}(\hat{x})$ & 0.033 & 1 & 0.300 & 0.776 & 0.700 \\
\hline $10 \mathrm{~h}(\hat{y})$ & 0.100 & 2 & 0.636 & 0.343 & 0.587 \\
\hline $10 \mathrm{~h}(\hat{x})$ & 0.056 & 2 & 0.354 & 0.757 & 0.682 \\
\hline $10 \mathrm{i}(\hat{y})$ & 0.178 & 3 & 0.924 & 0.622 & 0.491 \\
\hline $10 \mathrm{i}(\hat{x})$ & 0.211 & 3 & 1.097 & 0.414 & 0.434 \\
\hline $10 \mathrm{j}(\hat{y})$ & 0.067 & 1 & 0.600 & 0.727 & 0.600 \\
\hline $10 \mathrm{j}(\hat{x})$ & 0.100 & 1 & 0.900 & 0.376 & 0.499 \\
\hline
\end{tabular}


Table 5.2.2. Change point statistics, actual and predicted equivalent permeability for the 30 by 30 pixel patterns with $80 \%$ voids.

\begin{tabular}{|c|c|c|c|c|c|}
\hline Pattern & $C P$ & $F$ & $N C P$ & $\bar{K}$ & $\hat{K}_{e q}$ \\
\hline $30 a(\hat{y})$ & 0.109 & 12 & 0.914 & 0.580 & 0.495 \\
\hline 30a $(\hat{x})$ & 0.117 & 12 & 0.981 & 0.567 & 0.472 \\
\hline 30b (ŷ) & 0.132 & 16 & 0.958 & 0.427 & 0.480 \\
\hline $30 \mathrm{~b}(\hat{x})$ & 0.119 & 16 & 0.866 & 0.518 & 0.511 \\
\hline $30 c(\hat{y})$ & 0.113 & 16 & 0.816 & 0.510 & 0.527 \\
\hline $30 \mathrm{c}(\hat{x})$ & 0.126 & 16 & 0.916 & 0.507 & 0.494 \\
\hline $30 \mathrm{~d}(\hat{y})$ & 0.113 & 16 & 0.816 & 0.405 & 0.527 \\
\hline $30 \mathrm{~d}(\hat{x})$ & 0.101 & 16 & 0.733 & 0.486 & 0.555 \\
\hline 30e $(\hat{y})$ & 0.113 & 19 & 0.749 & 0.606 & 0.550 \\
\hline $30 \mathrm{e}(\hat{x})$ & 0.099 & 19 & 0.658 & 0.535 & 0.580 \\
\hline $30 f(\hat{y})$ & 0.126 & 18 & 0.864 & 0.512 & 0.511 \\
\hline $30 \mathrm{f}(\hat{x})$ & 0.132 & 18 & 0.904 & 0.496 & 0.498 \\
\hline $30 \mathrm{~g}(\hat{y})$ & 0.139 & 18 & 0.951 & 0.247 & 0.482 \\
\hline $30 \mathrm{~g}(\hat{x})$ & 0.135 & 18 & 0.919 & 0.486 & 0.493 \\
\hline 30h $(\hat{y})$ & 0.095 & 8 & 0.978 & 0.587 & 0.473 \\
\hline $30 \mathrm{~h}(\hat{x})$ & 0.106 & 8 & 1.084 & 0.431 & 0.438 \\
\hline $30 \mathrm{i}(\hat{y})$ & 0.147 & 16 & 1.066 & 0.553 & 0.444 \\
\hline $30 \mathrm{i}(\hat{x})$ & 0.144 & 16 & 1.042 & 0.422 & 0.452 \\
\hline $30 \mathrm{j}(\hat{y})$ & 0.108 & 12 & 0.904 & 0.566 & 0.498 \\
\hline $30 \mathrm{j}(\hat{x})$ & 0.115 & 12 & 0.962 & 0.425 & 0.479 \\
\hline 30k (̂े) & 0.105 & 12 & 0.876 & 0.419 & 0.508 \\
\hline 30k $(\hat{x})$ & 0.092 & 12 & 0.770 & 0.634 & 0.543 \\
\hline $30 \mathrm{I}(\hat{y})$ & 0.148 & 23 & 0.897 & 0.439 & 0.500 \\
\hline $301(\hat{x})$ & 0.159 & 23 & 0.959 & 0.525 & 0.486 \\
\hline $30 \mathrm{~m}(\hat{y})$ & 0.135 & 20 & 0.872 & 0.504 & 0.509 \\
\hline $30 \mathrm{~m}(\hat{x})$ & 0.148 & 20 & 0.962 & 0.561 & 0.479 \\
\hline
\end{tabular}

Figure 5.2.2 graphically demonstrates the correlation between equivalent permeability and the $N C P$.

\section{Prediction Model}

The equivalent permeability of a pattern with $80 \%$ voids is predicted in terms of $N C P$ as

$$
\dot{K}_{e q}=0.8-\beta N C P
$$

where $\beta$ is to be determined. Prior information is built into this model; for patterns with 0.80 void fractions where each row parallel to the applied pressure drop has 
Change Point Statistic and Keq

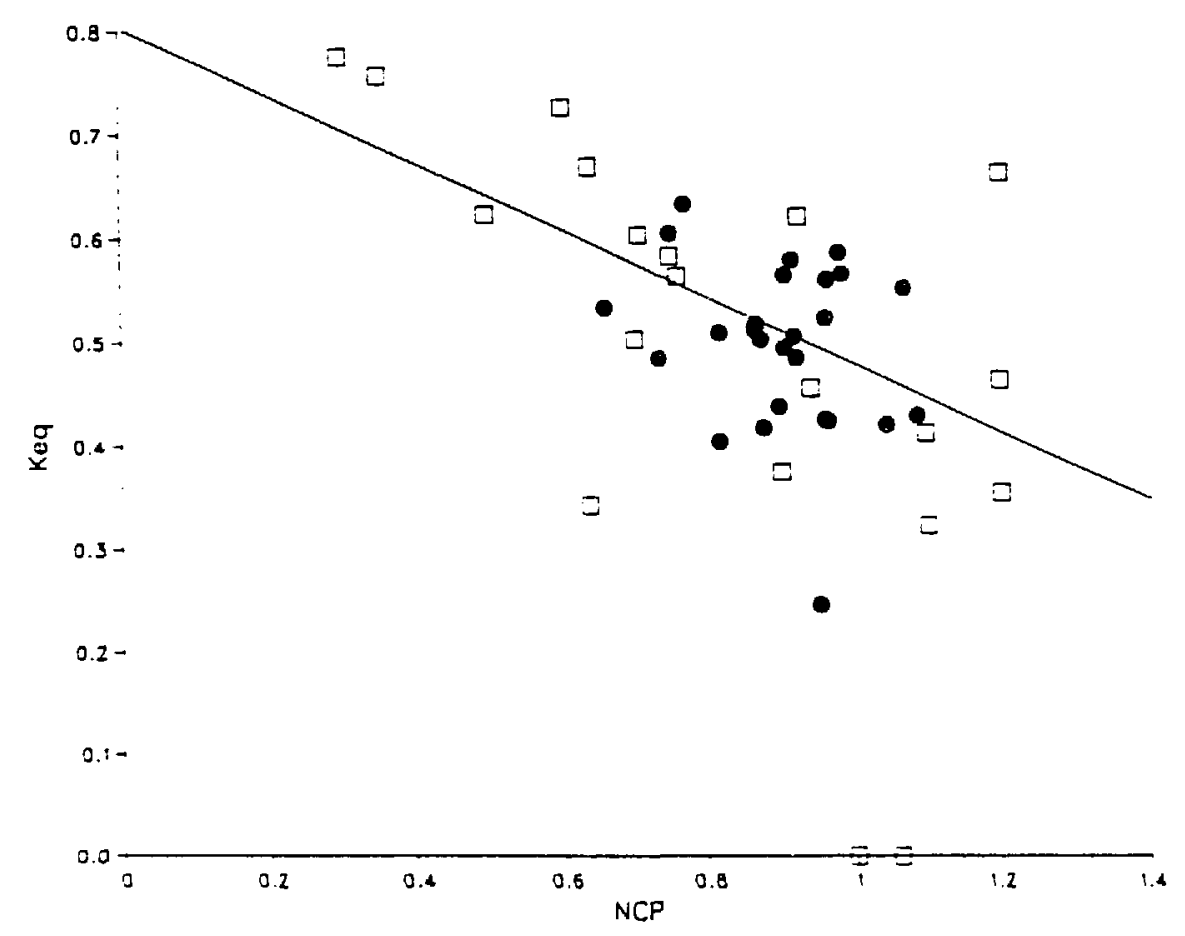

Figure 5.2.2 Equivalent permeability versus $N C P$ for 10 by 10 pixel and 30 by 30 pixel simulated patterns with $80 \%$ voids. Straight line is predicted equivalent permeability according to NCP model. 
either all voids or all contact areas, the model predicts equivalent permeability exactly. For instance, for the pattern shown in Figure 5.2.1, for a pressure drop in the $x$ direction, $N C P=0$ and both $\hat{K}_{e q}$ and $K_{e q}$ equal 0.80 .

A best fit value for $\beta$ was determined by fitting the prediction model to the 10 by 10 pixel patterns alone, the 30 by 30 pixel patterns alone and then patterns from both scales pooled together. $\hat{\beta}$ minimized

$$
R \hat{S} E=\left[\frac{1}{M-1} \sum_{i=1}^{M}\left(K_{e q}-\hat{K}_{e q}(\beta)\right)_{i}{ }^{2}\right]^{\frac{1}{2}}
$$

where $K_{\infty}$ and $\hat{K}_{a q}(\beta)$ denote actual and predicted equivalent permeability and $M$ is the number of data points in the fit. $M$ is 18 for the 10 by 10 pixel case, 26 for the 30 by 30 case, and 44 for the pooled case. Since, the 10 by 10 pixel patterns with $K_{\text {eq }}=0$ do not follow the linear trend set by the other patterns, they are excluded from the fit. Hence, the prediction model is intended to predict the flow through patterns with equivalent permeability in the range of the patterns included in the fit. No prediction is listed for the $K_{e q}=0$ data.

Table 5.2.3 shows that the best fit values of $\beta$ were nearly the same for both scales. In parentheses, the standard errors from the regression are listed.

Table 5.2.3. Fitted values of Change Point statistic model parameter and $R \hat{S E}$.

\begin{tabular}{|cccc|}
\hline Scale & $M$ & $\beta($ s.e. $)$ & $R \hat{S E}$ \\
\hline \hline 10 & 18 & $0.316(0.017)$ & 0.114 \\
30 & 26 & $0.334(0.017)$ & 0.081 \\
both & 44 & $0.327(0.016)$ & 0.095 \\
\hline
\end{tabular}

For the pattems under consideration, NCP is not greater than 1.1. Hence, using 0.316 instead of 0.334 changes the prediction by at most 0.02 . This is a minor difference. Moreover, the difference between the fited values of $\beta$ at the two scales is equal to 0.018 and the standard error reported for each fit is 0.017 . Thus, the difference is not 
statistically significant. As a caveat, to interpret the standard error as a precision for the estimated value of $\beta$, it is assumed that the prediction errors for each pattern $\left(K_{\text {eq }}-\hat{K}_{\text {sqq }}\right)$ have approximately normal, i.e. Gaussian, distributions with mean zero and constant variance. Since these assumptions need not be met, interpretation of the standard error is ambiguous.

Figure 5.2.2 also plots predicted equivalent permeability. To predict equivalent permeability, the 44 pattern fitted value, $\hat{\beta}=0.327$, was used. Tables 5.2.1 and 5.2.2 list both predicted and actual equivalent permeability for each pattern.

\section{Consistency Caveat}

By forcing the model to consistently predict the equivalent permeability of patterns with $N C P=0$, we might not be predicting the equivalent permeability of patterns with non-zero $N C P$ as well as possible. To explore this possibility, the model

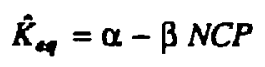

was fit to the 44 patterns by minimizing

$$
R \hat{S E}=\left[\frac{1}{44-2} \sum_{i=1}^{44}\left(K_{a q}-\hat{K}_{\mathrm{eq}}(\alpha, \beta)\right)_{i}^{2}\right]^{\frac{1}{2}}
$$

The best fit values of the parameters and the new $R \hat{S} E$ are listed in Table 5.2.4.

Table 5.2.4. Fitted model parameter values and $R \bar{S} E$ for inconsistent Change Point statistic model.

\begin{tabular}{|ccc|}
\hline$\alpha$ & $\beta($ s.e. $)$ & $R \hat{S E}$ \\
\hline \hline $0.791(.065)$ & $0.317(0.073)$ & 0.096 \\
\hline
\end{tabular}

Table 5.2.4 shows that adding the extra parameter did not improve prediction; $R \hat{S E}$ actually increased. Furthermore, the fitted value of $\alpha$ is close to 0.80 ; the difference between 0.791 and 0.800 is less than the reported standard error. Therefore, setting $\alpha$ to 0.80 is reasonable. 


\section{Summary}

For the simulated 10 by 10 and 30 by 30 pixel patterns with 0.80 void fractions, equivalent permeability was related to the normalized change point statistic. The following empirical model,

$$
\hat{K}_{e q}=p-\beta N C P,
$$

was fit to data from 10 by 10 pixel and 30 by 30 pixel simulated patterns with $80 \%$ voids. The fitted value of $\beta$ was nearly the same for both the 10 by 10 pixel and the 30 by 30 pixel scales. Moreover, NCP was shown to be discretization invariant, deterministic replication invariant for a special class of patterns and statistical replication invariant for simulated patterns from Chapter 3. Although $N C P$ is a very promising statistic, there is still work to do because it does not capture all possible information important for flow. For instance, NCP is insensitive to the relative location of contact area features.

As an illustration of this limitation of $N C P$, consider the two patterns shown in Figure 5.2.3. Although both patterns have the same $N C P$ statistic for a pressure drop in the $x$ direction, they surely have different equivalent permeabilities. Hence, NCP is not sufficient to predict flow exactly. In the next section, spatial statistics sensitive to both the shapes and the relative location of the features are presented. 

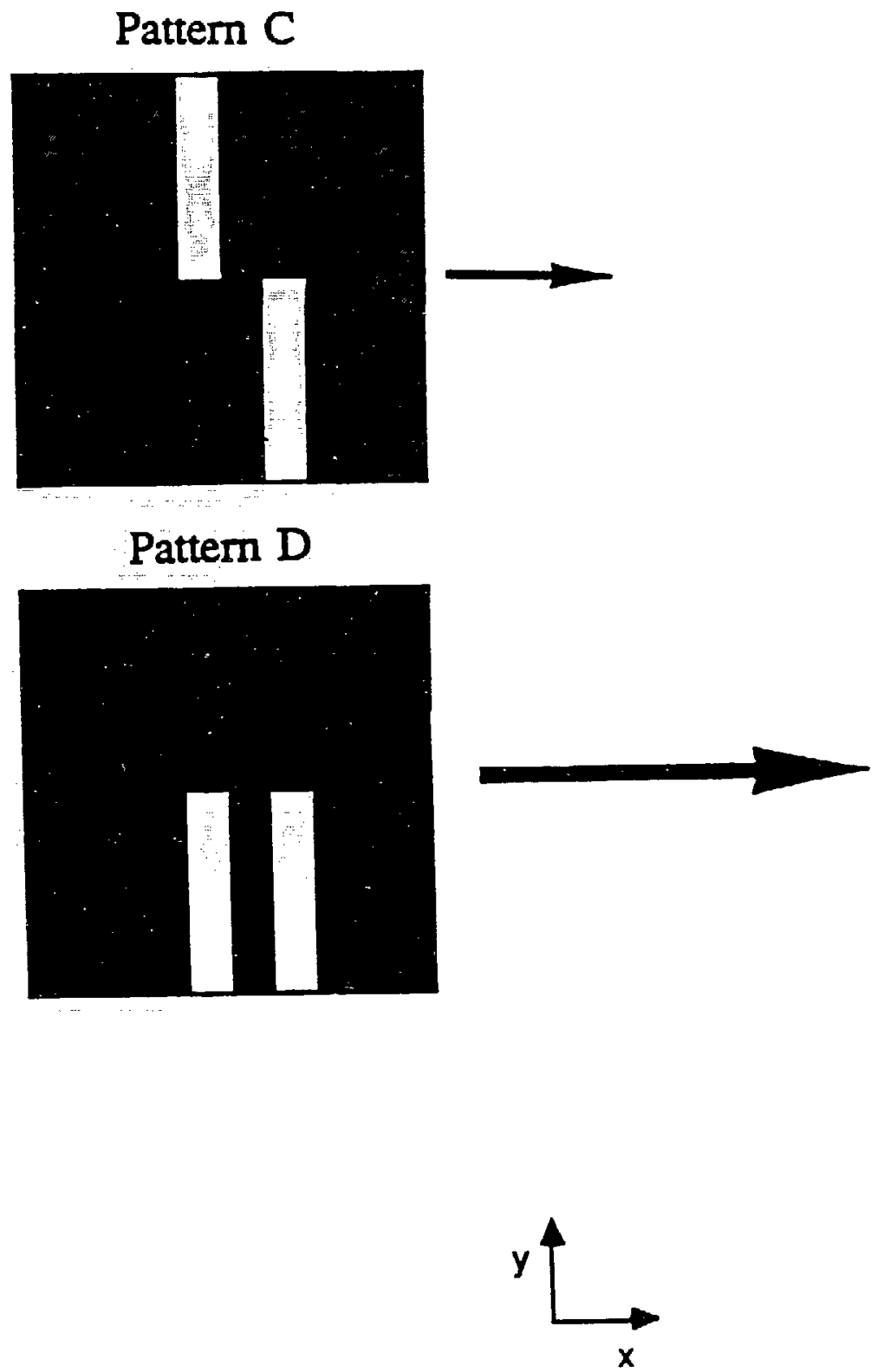

XBL $893-1048$

Figure 5.2.3 For pattems $\mathrm{C}$ and $\mathrm{D}$, for a pressure drop in the $x$ direction, NCP is the same even though the relative locations of the contact area features are different for each pattern. 


\subsection{Row and Column Dispersion Statistics}

\section{Introduction and Summary}

As noted in Section 5.2, the change point statistic relays information about shapes of contact area features, but misses information about the relative location of contact area features within a pattern. Here, two spatial statistics sensitive to feature shapes as well as the relative location of contact area features are examined. The spatial statistics measure the dispersion of void pixels in the rows and columns of a pattern. To illustrate dispersion, reconsider the pattern shown in Figure 5.2.1. In the rows which run in the $x$ direction, the dispersion of void pixels is maximal because each row is either $100 \%$ or $0 \%$ void. However in the columns which run in the $y$ direction, the dispersion of voids is minimal because each column has exactly the same fraction of voids $(0.80)$. When the dispersion of voids in the rows parallel to the pressure drop which drives the flow is maximum, flow is greatest. Similarly, when the dispersion of voids in the columns normal to the applied pressure drop is minimal, flow is greatest. For example, for the two patterns show in Figure 5.2.3, voids are more widely dispersed in the rows that run in the $x$ direction in pattern $D$ than in pattern $\mathrm{C}$. Hence, although $N C P$ is the same for each of these patterns, the extra information provided by the dispersion statistic should predict a higher equivalent permeability for pattern $\mathrm{D}$ (for a pressure drop in the $x$ direction). In this Chapter, flow through the simulazed 10 by 10 and 30 by 30 pixel patterns with 0.80 void fractions is related to the dispersion statistics.

\section{Statistics Defined}

To get a row dispersion statistic for a $N$ by $N$ pixel pattern, we first compute the fraction of voids in each row. Let $r_{1}$ denote the number of void pixels in the first row, $r_{2}$ be the number of voids in the second row,... and $r_{N}$ the number of voids in the $N^{\text {th }}$ row. From these row counts, a vector of void fractions is formed as follows 


$$
\left(\frac{r_{1}}{N}, \frac{r_{2}}{N}, \cdots \frac{r_{N}}{N}\right)
$$

With $p_{k}=\frac{r_{k}}{N}$ denoting the void fraction of the $k^{\text {ck }}$ row, the variance of the row fractions is defined as

$$
\sigma_{r f}^{2}=\frac{1}{N} \sum_{i=1}^{N}\left[p_{i}-\frac{1}{N} \sum_{j=1}^{N} p_{j}\right]^{2}
$$

In general, for a pattern with void fraction $p$, the maximum value that $\sigma_{r f}$ can take is $\sqrt{p(I-p)}$. This is the standard deviation of a $N$ by $N$ pattern where a fraction $p$ of the rows have void fraction equal to unity and the rest of the rows have void fraction equal to zero. For example, for the pattern shown in Figure 5.2.1, for a pressure drop in the $x$ direction, eight rows have void fraction equal to unity and two rows have void fractions equal to zero. Hence, for this pattem,

$$
\sigma_{r f}=\sqrt{\frac{1}{10}\left(8(1-.8)^{2}+2(0-.8)^{2}\right)}=\sqrt{.8(1-.8)}=0.4 .
$$

The explicit dependence on void fraction is removed as follows

$$
S I G R=\frac{\sigma_{r f}}{\sqrt{p(1-p)}}
$$

Thus, SIGR ranges from zero to unity. In exactly the same way as SIGR was computed from the row void fractions, a column dispersion statistic SIGC is computed from the column void fractions.

Both SIGR and SIGC are discretization invariant. To illustrate this, consider a simple 2 by 2 pixel patrem with row void fractions of $(1.0,0.5)$. If the same pattern is described with pixels one half as long, the new 4 by 4 pixel pattern has row void fractions of $(1.0,1.0,0.5,0.5)$. Both the 2 by 2 and 4 by 4 patterns have the same row fraction standard deviation and hence the same SIGR.

However, SIGC and SIGR are not statistical replication invariant. To demonstrate this, suppose each pixel in a $N$ by $N$ pixel pattern is independently assigned as void 
with probability $p$ and contact area with probability $1-p$. As $N$ increases, both $\sigma_{r f}$ and $\sigma_{c f}$ tend to $\frac{\sqrt{p(1-p)}}{\sqrt{N}}$ because the number of voids in a row is a binomial distribution with mean $N p$ and variance $N p(1-p)$. Because the fraction of void pixels in a row is $\frac{1}{N}$ times the number of voids in a row, the variance of the row fraction is the row count variance $(N p(1-p))$ divided by $N^{2}$. Thus, as the size of one of the simulated pattern increases, SIGC and SIGR tend to the uninformative limit of zero.

Zero is uninformative because two infinitely large patterns with different equivalent permeabilities should have different dispersion statistics. For instance, suppose the independence assumption is relaxed and that neighboring pixels in rows are assigned as void or contact in a spatially dependent way which affects flow. Further, suppose that the range of correlation is finite; pixels far enough away are independent. For these kinds of spatially correlated patterns, both SIGC and SIGR asymptotically tend to zero regardless of the strength of the correlation. Since, equivalent permeability permeability surely depends on the strength of correlation, asymptotic limits of zero for SIGC and SIGR are uninformative.

To get statistics which converge to informative limits, SIGC and SIGR must be multiplied by a geometric factor proportional to $\sqrt{N}$. Now, for the $N$ by $N$ patterns patterns described above, the number of contact area features $F$, on average, grows as $N^{2}$. That is, $F=O\left(N^{2}\right)$. Thus, $F^{\frac{1}{4}}=O(\sqrt{N})$. Therefore,

$$
N S I G C=\operatorname{SIGC} F^{\frac{1}{4}}
$$

and

$$
N S I G R=\operatorname{SIGR} F^{\frac{1}{4}}
$$

are statistical replication invariant for these patterns. 
As a caveat, note that the dispersion statistics are not deterministic replication invariant for the replication family of pattems shown in Figure 5.1.2. For instance, for all three patterns shown in Figure 5.1.2, SIGC is the same, but the number of contact area features is different in each pattern. Hence, NSIGC is different for each pattem. Thus, any prediction model based on the dispersion statistics is intended for irregular patterns rather than for periodic regular patterns like the ones shown in 5.1.2. Next, for the 10 by 10 and 30 by 30 pixel simulated patterns, NSIGC and NSIGR are related to equivalent permeability.

\section{Simulated Patterns}

Tables 5.3.1 and 5.3.2 list the statistics and equivalent permeabilities of the simulated patterns. 
Table 5.3.1. Dispersion statistics, predicted and actual equivalent permeability for 10 by 10 pixel patterns with $80 \%$ voids.

\begin{tabular}{|c|c|c|c|c|c|c|c|}
\hline & SIGR & SIGC & $F$ & NSIGR & NIIGC & $K_{\text {eq }}$ & $\hat{K}_{e q}$ \\
\hline $10 \mathrm{a}(\hat{y})$ & 0.433 & 0.158 & 5 & 0.647 & 0.236 & 0.564 & 0.632 \\
\hline $10 \mathrm{a}(\hat{x})$ & 0.158 & 0.433 & 5 & 0.236 & 0.647 & 0.457 & 0.397 \\
\hline $10 \mathrm{~b}(\hat{y})$ & 0.296 & 0.661 & 2 & 0.352 & 0.787 & 0.356 & 0.388 \\
\hline $10 \mathrm{~b}(\hat{x})$ & 0.661 & 0.296 & 2 & 0.787 & 0.352 & 0.665 & $0.6 ?$ \\
\hline $10 c(\dot{y})$ & 0.224 & 0.622 & 2 & 0.266 & 0.740 & 0.000 & \\
\hline $10 \mathrm{c}(\hat{x})$ & 0.622 & 0.224 & 2 & 0.740 & 0.266 & 0.670 & 0.649 \\
\hline $\operatorname{lod}(\hat{y})$ & 0.559 & 0.524 & 1 & 0.559 & 0.524 & 0.465 & 0.523 \\
\hline $10 \mathrm{~d}(\hat{x})$ & 0.524 & 0.559 & 1 & 0.524 & 0.559 & 0.504 & 0.503 \\
\hline $10 \mathrm{e}(\hat{y})$ & 0.447 & 0.335 & 4 & 0.632 & 0.474 & 0.584 & 0.558 \\
\hline $10 \mathrm{e}(\hat{x})$ & 0.335 & 0.447 & 4 & 0.474 & 0.632 & 0.324 & 0.467 \\
\hline $10 \mathrm{f}(\hat{y})$ & 0.524 & 0.536 & 2 & 0.624 & 0.638 & 0.605 & 0.507 \\
\hline $10 f(\hat{x})$ & 0.536 & 0.524 & 2 & 0.638 & 0.624 & 0.624 & 0.515 \\
\hline $\log (\hat{y})$ & 0.112 & 0.942 & 1 & 0.112 & 0.942 & 0.000 & \\
\hline $10 \mathrm{~g}(\hat{x})$ & 0.942 & 0.112 & 1 & 0.942 & 0.112 & 0.776 & 0.751 \\
\hline $10 \mathrm{~h}(\hat{y})$ & 0.224 & 0.844 & 2 & 0.266 & 1.004 & 0.343 & 0.300 \\
\hline $10 \mathrm{~h}(\hat{x})$ & 0.844 & 0.224 & 2 & 1.004 & 0.266 & 0.757 & 0.722 \\
\hline $10 \mathrm{i}(\hat{y})$ & 0.500 & 0.112 & 3 & 0.658 & 0.147 & 0.622 & 0.662 \\
\hline $10 \mathrm{i}(\dot{x})$ & 0.112 & 0.500 & 3 & 0.147 & 0.658 & 0.414 & 0.369 \\
\hline $10 \mathrm{j}(\hat{y})$ & 0.783 & 0.296 & 1 & 0.783 & 0.296 & 0.727 & 0.652 \\
\hline $10 \mathrm{j}(\hat{x})$ & 0.296 & 0.783 & 1 & 0.296 & 0.783 & 0.376 & 0.373 \\
\hline
\end{tabular}


Table 5.3.2. Dispersion statistics, predicted and actual equivalent permeability for 30 by 30 pixel patterns with $80 \%$ voids.

\begin{tabular}{|c|c|c|c|c|c|c|c|}
\hline & $S I G R$ & $S I G C$ & $F$ & NSIGR & $N S I G C$ & $K_{e q}$ & $\hat{K}_{a q}$ \\
\hline $30 a(\hat{y})$ & 0.278 & 0.192 & 12 & 0.518 & 0.358 & 0.580 & 0.561 \\
\hline $30 \mathrm{a}(\hat{x})$ & 0.192 & 0.278 & 12 & 0.358 & 0.518 & 0.567 & 0.469 \\
\hline $30 b(\hat{y})$ & 0.289 & 0.224 & 16 & 0.578 & 0.449 & 0.427 & 0.550 \\
\hline $30 \mathrm{~b}(\hat{x})$ & 0.224 & 0.289 & 16 & 0.449 & 0.578 & 0.518 & $0.4^{\prime}$ 'ó \\
\hline $30 c(\hat{y})$ & 0.305 & 0.409 & 16 & 0.610 & 0.819 & 0.510 & 0.450 \\
\hline $30 c(\hat{x})$ & 0.409 & 0.305 & 16 & 0.819 & 0.610 & 0.507 & 0.569 \\
\hline $30 \mathrm{~d}(\hat{\mathrm{y}})$ & 0.243 & 0.459 & 16 & 0.487 & 0.919 & 0.405 & 0.386 \\
\hline $30 \mathrm{~d}(\hat{x})$ & 0.459 & 0.243 & 16 & 0.919 & 0.487 & 0.486 & 0.633 \\
\hline $30 e(\hat{y})$ & 0.393 & 0.287 & 19 & 0.821 & 0.599 & 0.606 & 0.573 \\
\hline $30 \mathbf{e}(\hat{x})$ & 0.287 & 0.393 & 19 & 0.599 & 0.821 & 0.535 & 0.446 \\
\hline $30 f(\hat{y})$ & 0.330 & 0.305 & 18 & 0.680 & 0.629 & 0.512 & 0.525 \\
\hline $30 f(x)$ & 0.305 & 0.330 & 18 & 0.629 & 0.680 & 0.496 & 0.496 \\
\hline $30 \mathrm{~g}(\hat{y})$ & 0.212 & 0.297 & 18 & 0.436 & 0.613 & 0.247 & 0.463 \\
\hline $30 \mathrm{~g}(\hat{x})$ & 0.297 & 0.212 & 18 & 0.613 & 0.436 & 0.486 & 0.563 \\
\hline 30h $(\dot{y})$ & 0.451 & 0.365 & 8 & 0.758 & 0.613 & 0.587 & 0.551 \\
\hline 30h $(\hat{x})$ & 0.365 & 0.451 & 8 & 0.613 & 0.758 & 0.431 & 0.469 \\
\hline $30 \mathrm{i}(\hat{y})$ & 0.371 & 0.316 & 16 & 0.743 & 0.631 & 0.553 & 0.542 \\
\hline $30 \mathrm{i}(\hat{x})$ & 0.316 & 0.371 & 16 & 0.631 & 0.743 & 0.422 & 0.478 \\
\hline $30 \mathrm{j}(\hat{y})$ & 0.298 & 0.272 & 12 & 0.555 & 0.506 & 0.566 & 0.527 \\
\hline $30 \mathrm{j}(\hat{x})$ & 0.272 & 0.298 & 12 & 0.506 & 0.555 & 0.425 & 0.499 \\
\hline 30k $(\hat{y})$ & 0.276 & 0.521 & 12 & 0.514 & 0.970 & 0.419 & 0.378 \\
\hline 30k $(\hat{x})$ & 0.521 & 0.276 & 12 & 0.970 & 0.514 & 0.634 & 0.640 \\
\hline $301(\hat{y})$ & 0.342 & 0.356 & 23 & 0.748 & 0.779 & 0.439 & 0.500 \\
\hline $301(\hat{x})$ & 0.356 & 0.342 & 23 & 0.779 & 0.748 & 0.525 & 0.517 \\
\hline $30 \mathrm{~m}(\hat{y})$ & 0.263 & 0.239 & 20 & 0.556 & 0.505 & 0.504 & 0.528 \\
\hline $30 \mathrm{~m}(\hat{x})$ & 0.239 & 0.263 & 20 & 0.505 & 0.556 & 0.561 & 0.498 \\
\hline
\end{tabular}

Figure 5.3.1 and 5.3.2 show a correlation between the dispersion statistics and flow. Besides being correlated with flow, Figure 5.3.3 shows that NSIGC and NSIGR are correlated with each other. However, the statistics are not obviously colinear, there is a lot of scatter about the linear trend between NSIGR and NSIGC. Hence, the statistics are not obvious!y redundant. Next, equivalent permeability is predicted in terms of the dispersion statistics. 
Column Dispersion statistic and Keq

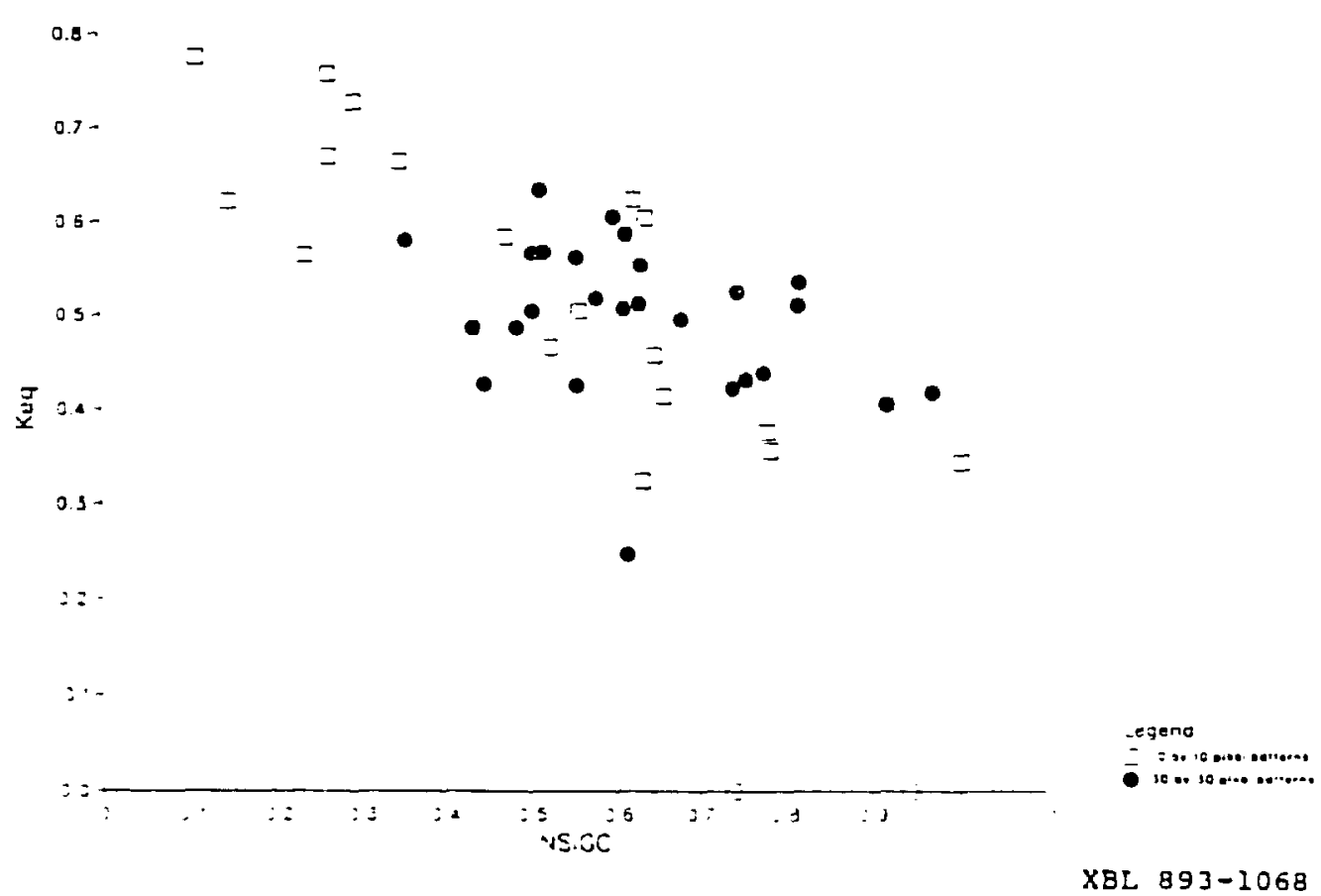

Figure 5.3.1 Equivalent permeability versus column dispersion statistic (NSIGC) for 10 by 10 pixel and 30 by 30 pixel simulated patterns with $80 \%$ voids. 
Row Dispersion statistic and Keq

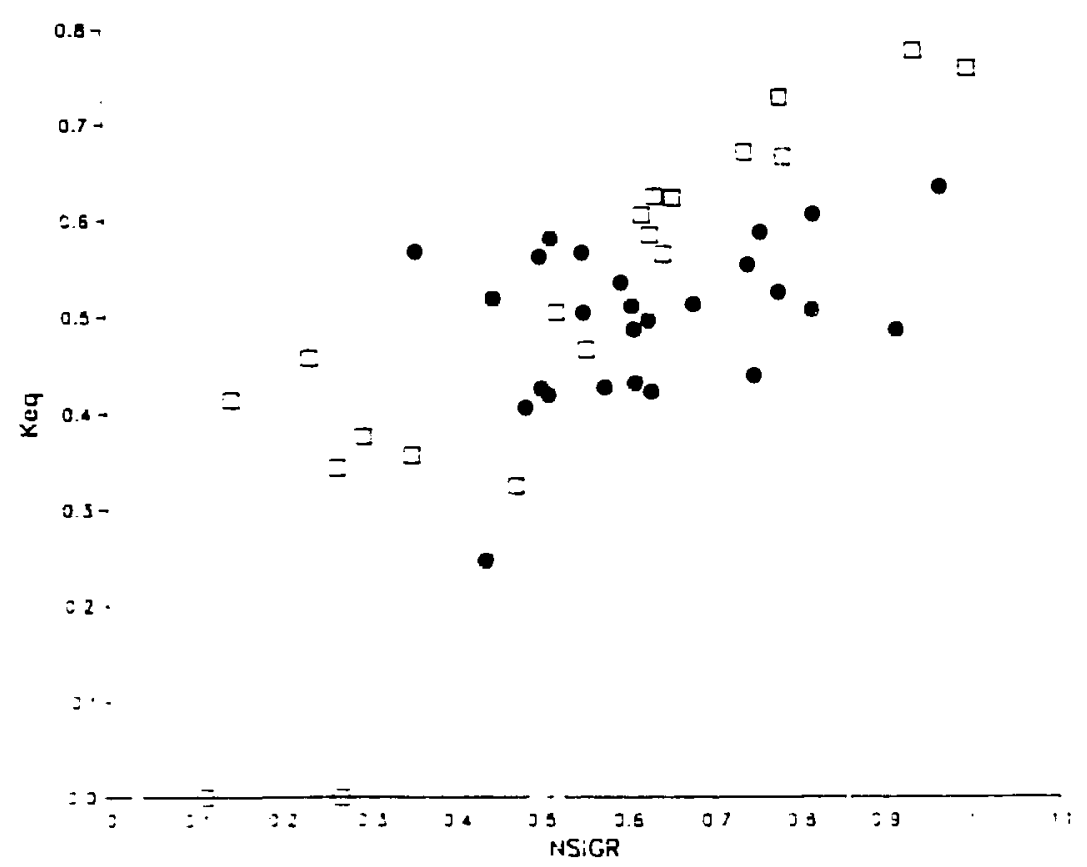

Figure 5.3.2 Equivalent permeability versus row dispersion statistic (NSIGR) for 10 by 10 pixel and 30 by 30 pixel simulated patterns with $80 \%$ voids. 


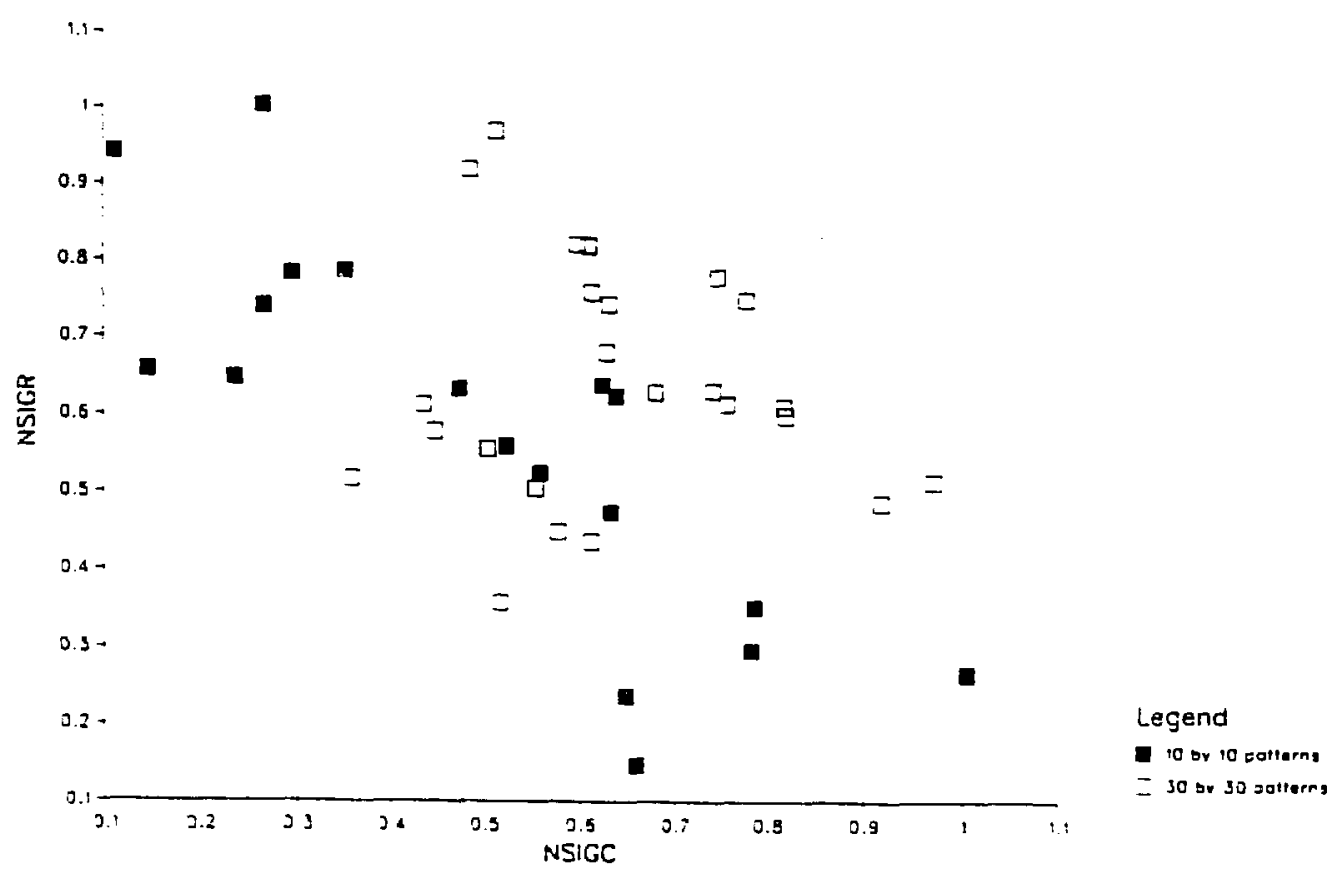

XBL $893-1070$

Figure 5.3.3 Row dispersion statistic (NSIGR) versus column dispersion statistic (NSIGC) for 10 by 10 pixel and 30 by 30 pixel patterns. Pattems with zero equivalent permeability are not plotted. 
Prediction Model for $K_{\mathrm{eq}}$

Since Figures 5.3.1 and 5.3.2 show linear trends between equivalent permeability and each of the dispersion statistics, we make the parsimonious choice of predicting equivalent permeability as

$$
\hat{K}_{a q}=0.8-\beta_{c}(N S I G C)+\beta_{r}(N S I G R-1)
$$

where $\beta_{c}$ and $\beta_{r}$ are empirical parameters to be determined. The model is parsimonious because it is linear in both NSIGC and NSIGR and because it consistently predicts equivalent permeability for patterns where each row has void fraction equal to zero or unity. For instance, for the pattem shown in Figure 5.2.1, for a pressure drop in the $x$ direction, NSIGC equals zero, NSIGR is unity and both actual and predicted equivalent permeability are 0.80 . As a caveat, the prediction model which best predicts the equivalent permeability of the simulated patterns need not be linear in NSIGC and NSIGR nor consistent. Later, the consistency issue is addressed. We do not explore the non-linearity issue.

The best fit values of $\beta_{r}$ and $\beta_{c}$ minimize

$$
R \hat{S E}=\left[\frac{1}{M-2} \sum_{i=1}^{M}\left(K_{e q}-\hat{K}_{\infty}\left(\hat{\beta}_{e}, \hat{\beta}_{r}\right)\right)^{2}\right]^{\frac{1}{2}}
$$

where $M$ is the number of data points in the fit. The Model was fit to the 10 by 10 pixel patterns, the 30 by 30 pixel patterns and then both scales together. Like in the previous section, $K_{\text {eq }}=0$ data points were excluded from the fit because they do not follow the linear trends set by the other data points. Table 5.3.3 shows how RSE and the fitted parameters depended on scale. In Tables 5.3.1 and 5.3.2, equivalent permeability is predicted using the 44 pattern fitted values, i.e.

$$
\hat{K}_{\text {eq }}=.8-.296 \text { NSIGC }+0.277(N S I G R-1) .
$$

Figure 5.3.4 plots predicted equivalent permeability versus actual equivalent permeability for all the patterns. 
Dispersion Statistics Model

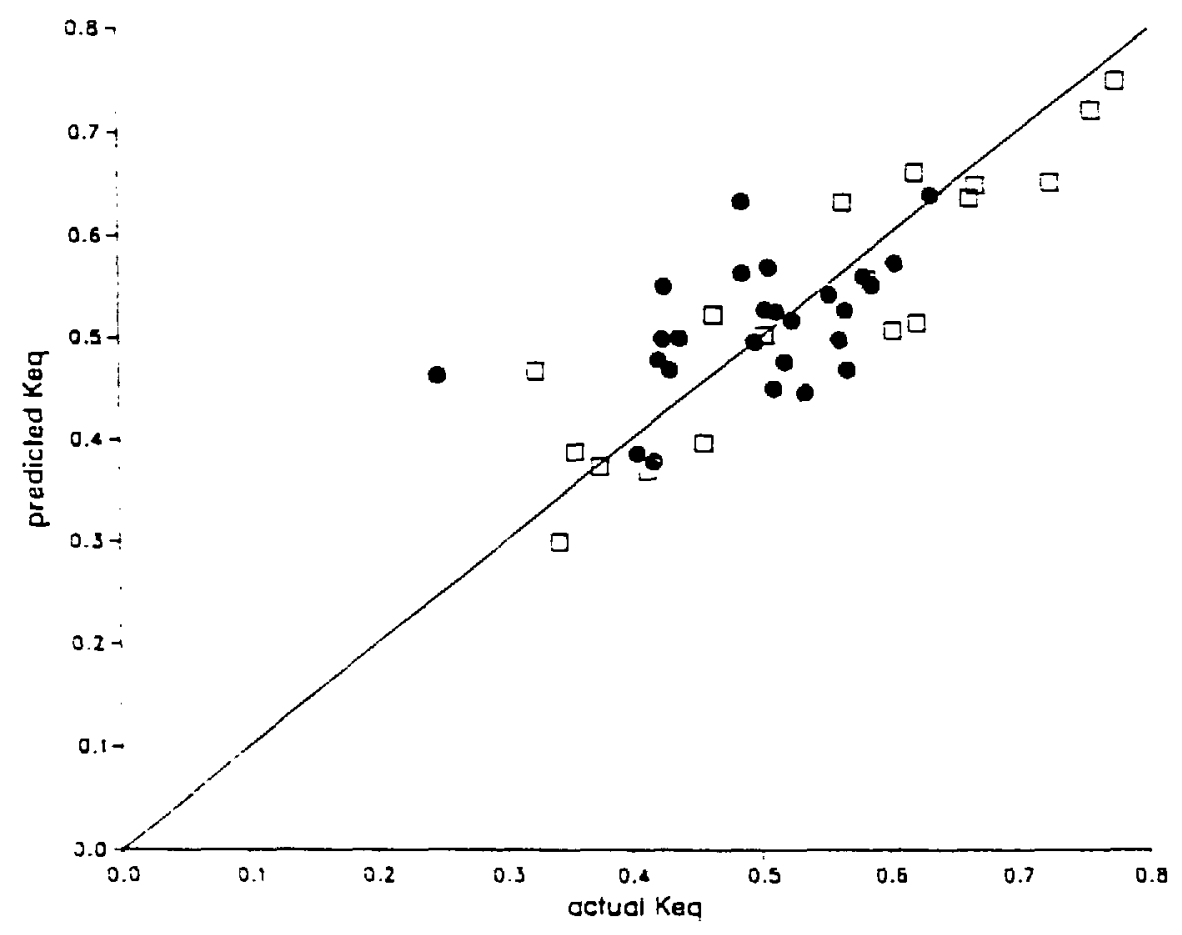

Figure 5.3.4 Predicted equivalent permeability according to dispersion statistic model versus actual equivalent permeability for the 10 by 10 pixel and 30 by 30 pixel pattems with $80 \%$ void fractions. 
Table 5.3 : Fitted parameters and root mean square prediction error for dispersion statistic prediction model.

\begin{tabular}{|ccccc|}
\hline Scale & $M$ & $\beta_{c}($ s.e. $)$ & $\beta_{r}($ s.e $)$ & $R \hat{S E}$ \\
\hline \hline 10 & 18 & $0.164(0.093)$ & $0.404(0.105)$ & 0.061 \\
30 & 26 & $0.311(0.055)$ & $0.273(0.089)$ & 0.076 \\
both & 44 & $0.296(0.043)$ & $0.277(0.060)$ & 0.071 \\
\hline
\end{tabular}

Scale effects

Note that when the model is fit to all the data, the fitted values of $\beta_{c}$ and $\beta_{r}$ are only slightly different from the values of $\beta_{c}$ and $\beta_{r}$ that result from fitting the model to the 30 by 30 pixel patterns. This suggests that the 44 pattern fitted values of the model parameters are appropriate for both the 10 by 10 pixel and 30 by 30 pixel patterns. Furthermore, the standard errors (s.e) are large enough so that the difference between fitted parameter observed at the two scales may not be statistically significant. That is, the differences could be due to fitting the model to an insufficient amount of data.

To determine if the fitted values of $\beta_{c}$ are significantly different at the two scales, the standard procedure is to form the ratio of the difference of the fitted parameters and the estimate of the standard error of the difference. Thus, the test statistic for determining if the fitted values of $\beta_{c}$ at the 10 by 10 and 30 by 30 scales are statistically significant is

$$
\frac{|0.311-0.164|}{\sqrt{\left(0.055^{2}+0.093^{2}\right)}}=1.36
$$

By similar calculation, the test statistic for determining if the fitted values of $\beta_{r}$ are significantly different at the two scales is 0.95 . According to standard hypothesis testing theory (Mood et al, 1974), if there is no scale effect, the above test statistics are to a good approximation, random realizations from a normal distribution with mean zero and variance one provided that the residuals about the fit are independently distributed, have mean zero, equal variances, and are approximately Gaussian. As a 
caveat, if these assumptions are not be obeyed by the data, the result of a hypothesis test is ambiguous. Outside of the textbook, this can easily happen. For instance, if the data follows a nonlinear trend in some predictor and a linear model is fit, the residuals will not have mean zero. With the caveat noted, the null hypothesis that $\beta_{c}$ is the same at the 10 by 10 pixel and 30 by 30 pixel scales, i.e.

$$
H_{o}: \beta_{c_{10}}=\beta_{c_{30}}
$$

is rejected with only $82 \%$ confidence because the probability that a random realization of a Gaussian with mean one and variance one having absolute value greater than or equal to 1.36 is $18 \%$. By similar calculation, the hypothesis that $\beta_{r}$ is not dependent on scale is rejected at a confidence level of only $66 \%$. Hence, hypothesis testing does not provide strong evidence that the "true" model parameters, i.e. the fitted values obtained from fitting the model to millions of statistically similar data points, are scale dependent.

Lastly, when the equivalent permeabilities of the 10 by 10 patterns were predicted using the 44 pattem fitted values for $\beta_{c}$ and $\beta_{r}, R \hat{S E}$ was nearly the same as when using the parameters from the fit using only the 10 by 10 patterns. $R \hat{S} E$ increased from 0.060 to only 0.066 . This supports the claim that the 44 pattern fitted values do a good job for both scales.

\section{Consistency Caveat}

By forcing the prediction model to consistently predict the equivalent permeability of patterns like the one shown in figure 5.2.1, the equivalent permeability of other patterns may not be predicted as well as possible. It is possible that a model which is linear in the dispersion statistics but inconsistent, might predict the equivalent permeability of the simulated patterns better. To explore this possibility, the model,

$$
\hat{K}_{a}=\alpha+\beta_{c} N S I G C-\beta_{r}(N S I G R-1)
$$

was fit to data from the 44 patterns by minimizing 


$$
R \hat{S E}=\left[\frac{1}{44-3} \sum_{i=1}^{44}\left(K_{e q}-\hat{K}_{e q}\right)^{2}\right]^{\frac{1}{2}}
$$

Table 5.3.4 shows that $\alpha$ is close to 0.80 and that the new $R \hat{S} E$ is the same as the $R \hat{S} E$ for the consistent model. Hence, relaxing the consistency constraint does not improve prediction. Therefore, setting $\alpha=0.80$ is reasonable.

Table 5.3.4. Fitted parameters and root mean square prediction error for inconsistent dispersion statistic prediction model.

\begin{tabular}{|cccccc|}
\hline Scale & $M$ & $\alpha$ & $\beta_{c}($ s.e. $)$ & $\beta_{r}(s . e)$ & $R \hat{S E}$ \\
\hline \hline both & 44 & $0.776(0.034)$ & $0.267(0.060)$ & $0.265(0.063)$ & 0.071 \\
\hline
\end{tabular}

\section{Summary}

Statistical replication invariant and discretization invariant dispersion statistics were presented. The equivalent permeability of the 10 by 10 pixel and 30 by 30 pixel simulated patterns was predicted in terms of the row and column dispersion statistics. The fitted parameter values from fitting the model to data from both scales were close to the fitted parameters from fitting the model to only the 30 by 30 pixel patterns. Furthermore, using the fitted parameters from fitting the model to the pooled data, the 10 by 10 pixel pattem equivalent permeabilities were predicted almost as well as when using the fitted parameters from fitting the model to only the 10 by 10 patterns. This is good because we would like one prediction model to work for many scales. 


\subsection{EROSION STATISTIC}

\section{Introduction and Summary}

How well contact areas block flow partly depends on the width of flow channels between contact area features. Narrow channels bottleneck fluid flow like narrow roads bottleneck traffic flow. This section presents a spatial statistic which measures the width of flow channels between contact area features. To motivate the statistic, we show how the narrowest width of flow channel determines flow for patterns with trapezoidal shaped void regions. The equivalent permeabilities of the 10 by 10 and 30 by 30 pixel patterns are predicted in terms of a spatial statistic based on erosion (Serra, 1982) transformations of each pattern.

\section{Motivation}

Consider patterns with trapezoidal shaped void regions as shown in Figure 5.4.1. All of these square pattems have side length $l$ but different $l_{0}$ and $l_{1}$. Without loss of generality, the smaller side is always $l_{1}$. Void fraction is related to these lengths as

$$
p=\frac{l_{0}+l_{1}}{2 l}
$$

That is, void fraction is the ratio of the average width of the channel $\left(\frac{l_{0}+l_{1}}{2}\right)$ and the length of the pattern (l). Voids are assumed to have constant aperture $b$.

For flow across the trapezoidal pattern due to a pressure drop in the $x$ direction, the continuity equation for flow in the cubic law flow model (Eq. 4.8) is approximated by a one-dimensional continuity equation

$$
\frac{\partial}{\partial x}\left(q_{x} A\right)=\frac{\partial}{\partial x}\left(A(x) \frac{\partial}{\partial x} P(x)\right)=0
$$

where $P$ is pressure, $q=\left(q_{x}, 0,0\right)$ is the flow per unit area and $A(x)$ is channel width. Within the void region, permeability equals a positive constant $(K)$. Outside the void 

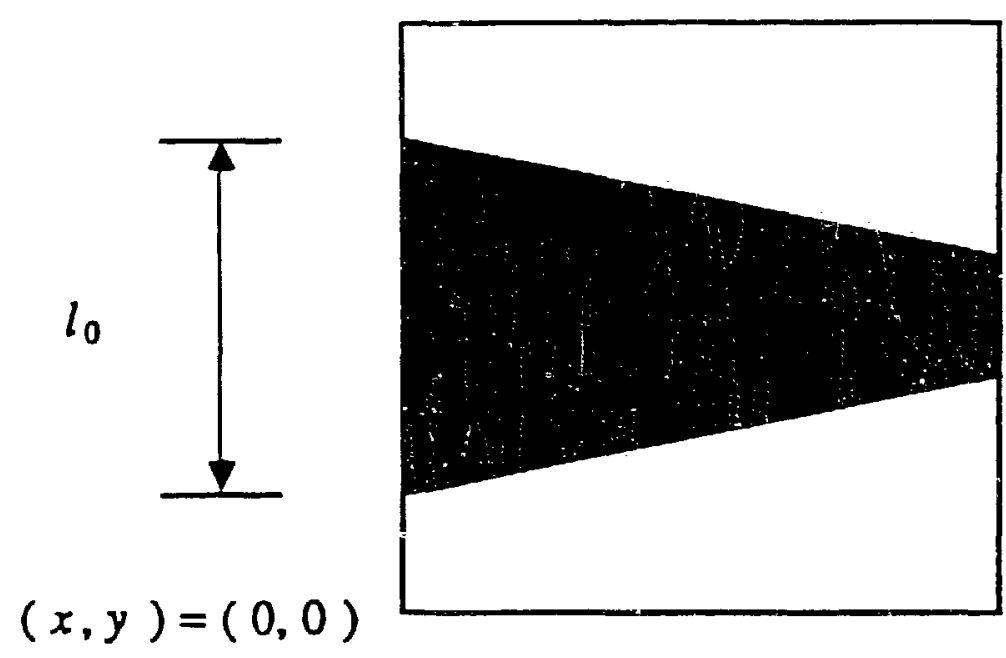

$$
(x, y)=(l, l)
$$
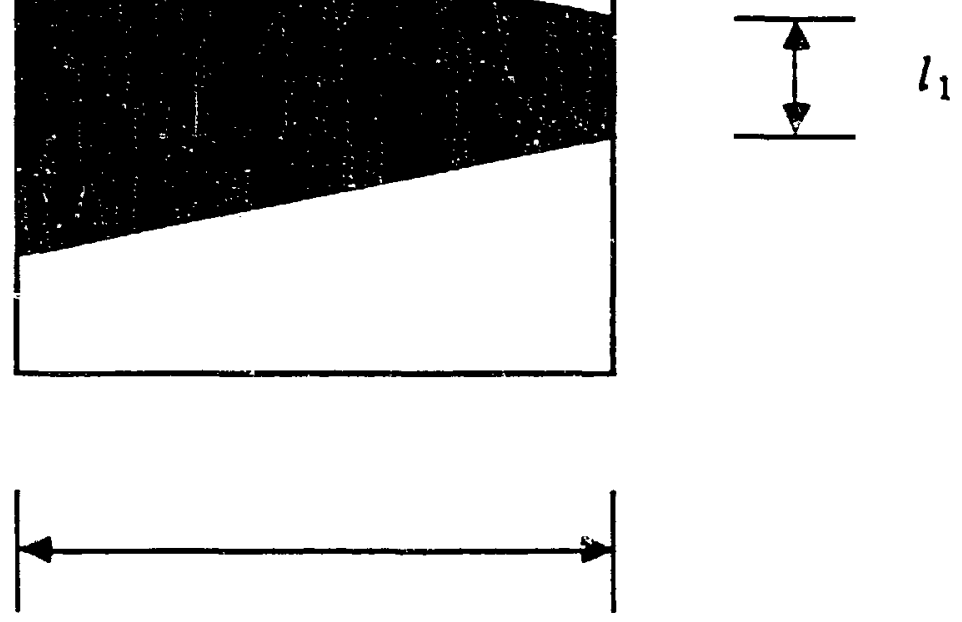

$$
l
$$

XBL 893-1072

Figure 5.4.1 Trapezoidal pattem. Void region is black. Contact areas are white. 
region, permeability is zero. This one dimensional flow model is called a fin approximation (Holman, 1976). The fin approximation ignores local fluid velocities and local pressure gradients in the direction normal to the applied pressure drop.

Assuming the above one-dimensional flow model, the flow through the rapezoidal patterns is $Q=-b l_{0} \frac{K}{\mu} \dot{P}(0)$ where $\mu$ is fluid viscosity and $\dot{P}(0)$ is the derivative of pressure at $x=0$. Since the channel width $A$ varies as

$$
A(x)=l_{0}+\left(l_{1}-l_{0}\right) \frac{x}{!}
$$

Eq. 5.4.2 can be written as

$$
\dot{A} \dot{P}+A \ddot{P}=0 \text {. }
$$

For the boundary conditions,

$$
P(0)=P_{1}+\Delta P
$$

and

$$
P(l)=P_{l}
$$

solution of Eq. 5.4.4 gives:

$$
P(x)=P_{1}+\frac{\Delta P}{\ln \frac{l_{0}}{l_{1}}} \ln \left(\frac{l_{0}}{l_{1}}+\left(1-\frac{l_{0}}{l_{1}}\right) \frac{x}{l}\right)
$$

Flow is maximum when $l_{0}$ and $l_{1}$ equal $l$, that is if void fraction is unity. For this case, the pressure gradient at $x=0$ is $-\frac{\Delta P}{l}$ and therefore

$$
Q_{\max }=b l_{0} \frac{K}{\mu} \frac{\Delta P}{l}=b \frac{K}{\mu} \Delta P .
$$

Thus,

$$
K_{\text {eq }}=\frac{Q}{Q_{\max }}=-\frac{l_{0} \dot{P}(0)}{\Delta P}
$$


Doing some algebra and calculus gives,

$$
K_{\text {eq }}=\frac{l_{0}-l_{1}}{l \log \frac{l_{0}}{l_{1}}} .
$$

Eq. 5.4.1 and 5.4.9 imply that

$$
K_{a q}=\frac{2\left(p-\frac{l_{1}}{l}\right)}{\log \left(\frac{2 p l}{l_{1}}-1\right)} .
$$

Also,

$$
\lim _{\frac{l_{1}}{l} \rightarrow 0} K_{e q}=0
$$

and by L'Hospitals Rule,

$$
\lim _{\frac{l_{1}}{l} \rightarrow p} K_{a q}=p
$$

For trapezoids with void fraction 0.5 , Figure 5.4.2 shows that $K_{\text {sq }}$ is a monotonici.ly increasing function of $\left(\frac{l_{1}}{l}\right)$. Next, a statistic which measures this controlling channel width is defined.

\section{Statistic Defined}

The statistic is based on erosional transformations of the pattern (Serra, 1982) An erosion transformation thins out the flow path by peeling away voids layer by layer. Because any spatial statistic must depend on the direction of pressure drop, the erosion is done in a directional way. For instance, for a pressure drop in the $y$ direction, all voids which are bordered on the immediate right ( $x$ direction) by contact area pixels are transformed into contact areas. Void pixels on the far right boundary are transformed into contact areas in the first erosion. In the second step, the pattern resulting from the first erosion is eroded using the same rules. Afier enough successive erosions, the flow path across the patterns thins out too much and the connection 


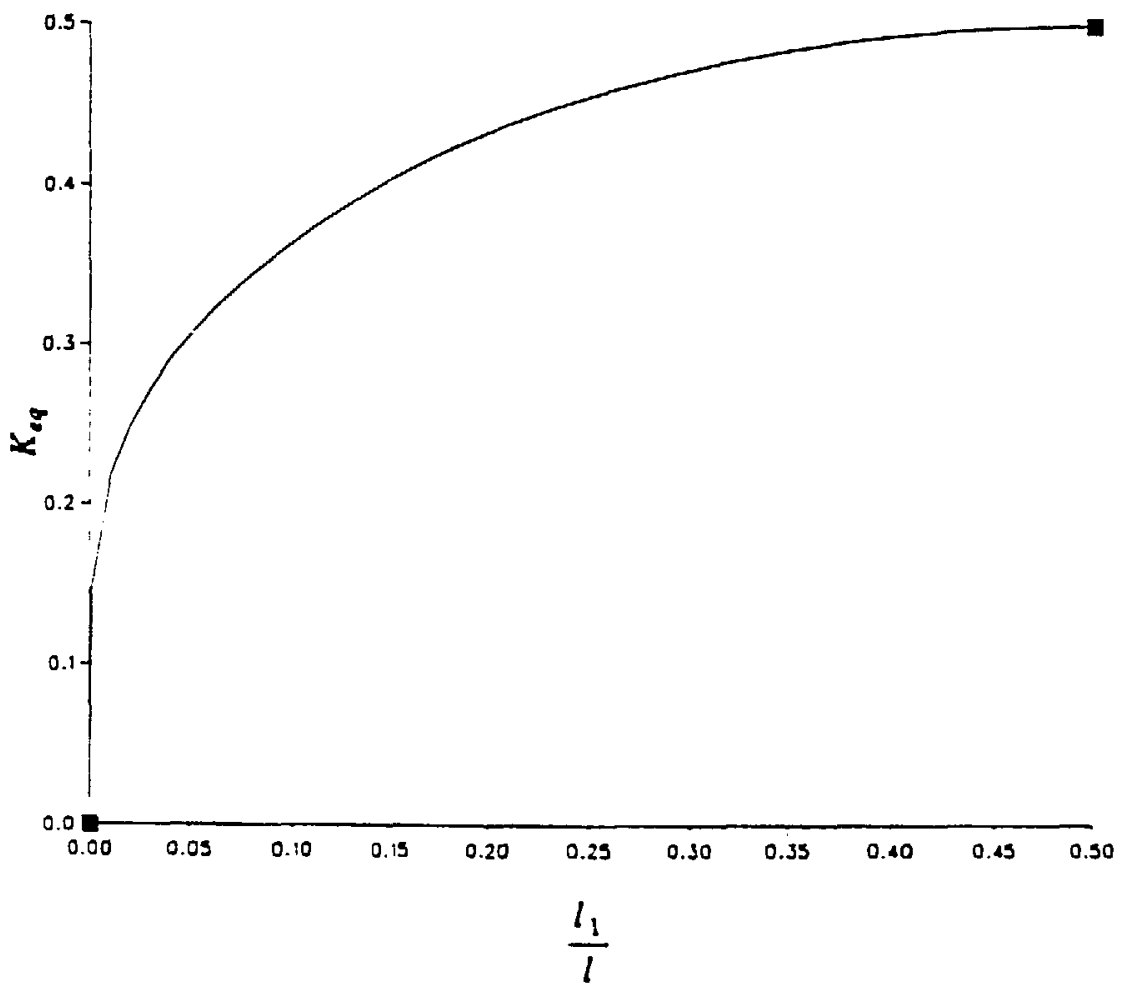

XBL 893-1073

Figure 5.4.2 Fin approximation for equivalent permeability of trapezoidal patterns with $50 \%$ contact area. 
of voids from inflow to outflow boundary is broken. Two void pixels are directly connected if they share a side. BREAK is the number of successive erosions necessary to break the connected path of voids from the inflow to outflow boundaries.

Figure 5.4.3 illustrates how erosion peels away voids from the flow path across a 10 by 10 pixel pattern. For a pressure drop in the $y$ direction, the inflow and outflow boundaries are disconnected after the third erosion. Hence, BREAK equals 3. Note, if the 10 by 10 pixel pattern were described with pixels half as long, BREAK would be twice as great (6). Thus, NBREAK is not discretization invariant. Next, the trapezoids are reconsidered so as to motivate a way to normalize BREAK .

So as to motivate a way to find a discretization invariant statistic, we note that for the trapezoids shown in Figure 5.4.1, the ratio $\frac{l_{1}}{l_{1}+l_{0}}$ and $p$ jointly determine $K_{e q}$. This ratio and $p$ do not depend on discreization. This suggests that BREAK which depends on $l_{1}$ and how finely the pattern is discretized, should be divided by the average width of the flow path $\frac{l_{1}+l_{0}}{2}$ (in pixel lengths uniss). The average width of the flow path in pixel lengths is also the average run length (RUN) of voids in the columns of the pattern. Thus, if the pattern is discretized so that $l_{1}$ is $k$ pixels lengths, and the average width of the channel $\left(\frac{l_{0}+l_{1}}{2}\right)$ is $m$ pixel lengths, $B R E A K=k$, $R U N$ is $m$ and $\frac{B R E A K}{R U N}$ equals $\frac{k}{m}$. Even though both BREAK and RUN depend on discretization, their ratio does not. Thus,

$$
\text { NBREAK }=\frac{B R E A K}{R U N}
$$

is discretization invariant for the trapezoidal patterns.

Although we have no proof that $N B R E A K$ is statistical replication invariant for general patterns, NBREAK is deterministic replication invariant for the family of pattems described below. In this family, the basic pattern is 10 by 10 pixels. Rows 1 


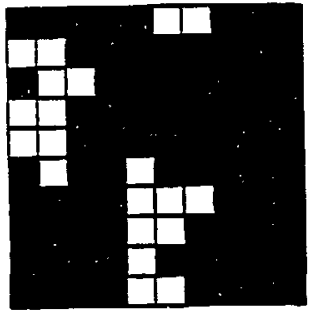

original pattern

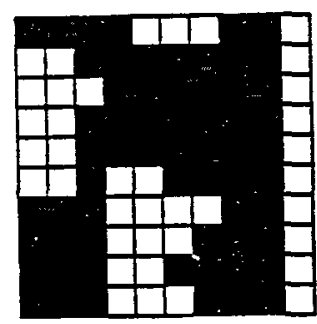

after 1 erosion

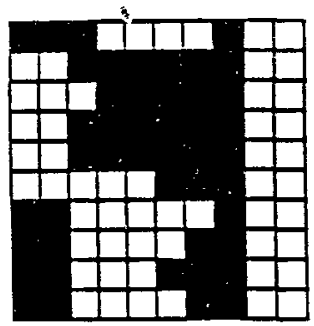

after 2 erosions

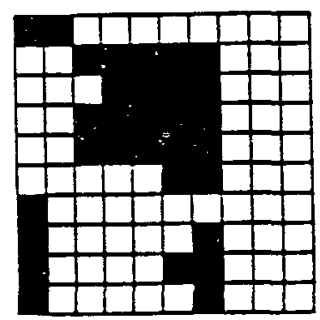

after 3 erosions

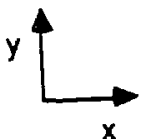

XBL $893-107 i$

Figure 5.4.3 Original 10 by 10 pixel pattem and eroded versions of it for a pressure drop in the $y$ direction. BREAK is three because it takes three erosions to disconnect the inflow and outflow boundaries. 
through 8 are filled with conducting voids and rows 9 and 10 are filled with nonconducting contact areas. Figure 5.4 .4 shows the basic pattern and the 2 by 2 and 8 by 8 replications of the basic pattern. For each of these patterns, $B R E A K=8, R U N=8$ and $N B R E A K=1$ for flow in the $x$ direction.

Coveats

For other families of patterns, NBREAK is not deterministic replication invariant. For instance, consider the replication family shown in Figure 5.4.5. Figure 5.4.5 shows a basic pattern and the 2 by 2 and 8 by 8 replications of the basic pattern. For either pressure drop direction, for the $k$ by $k$ member of the replication family, $R U N$ increases without limit as $k$ increases even though $B R E A K$ is independent of $k$. Hence, $\frac{B R E A K}{R U N}$ goes to zero as $k$ goes to infinity. Therefore, NBREAK is not deterministic replication invariant for this family of patterns.

Run length normalization failed to make sense for the patterns in Figure 5.4.5 because certain columns were $100 \%$ void and RUN did not converge as $k$ increased. But for the simulated patterns from Chapter 3, Appendix A shows that RUN converges to an asymptotic limit of approximately 12.6 pixels lengths. Hence, run length normalization makes sense for patterns simulated using the Chapter 3 model. Next, the equivalent permeabilities of the 10 by 10 and 30 by 30 pixel simulated patterns are predicted in terms of NBREAK. 

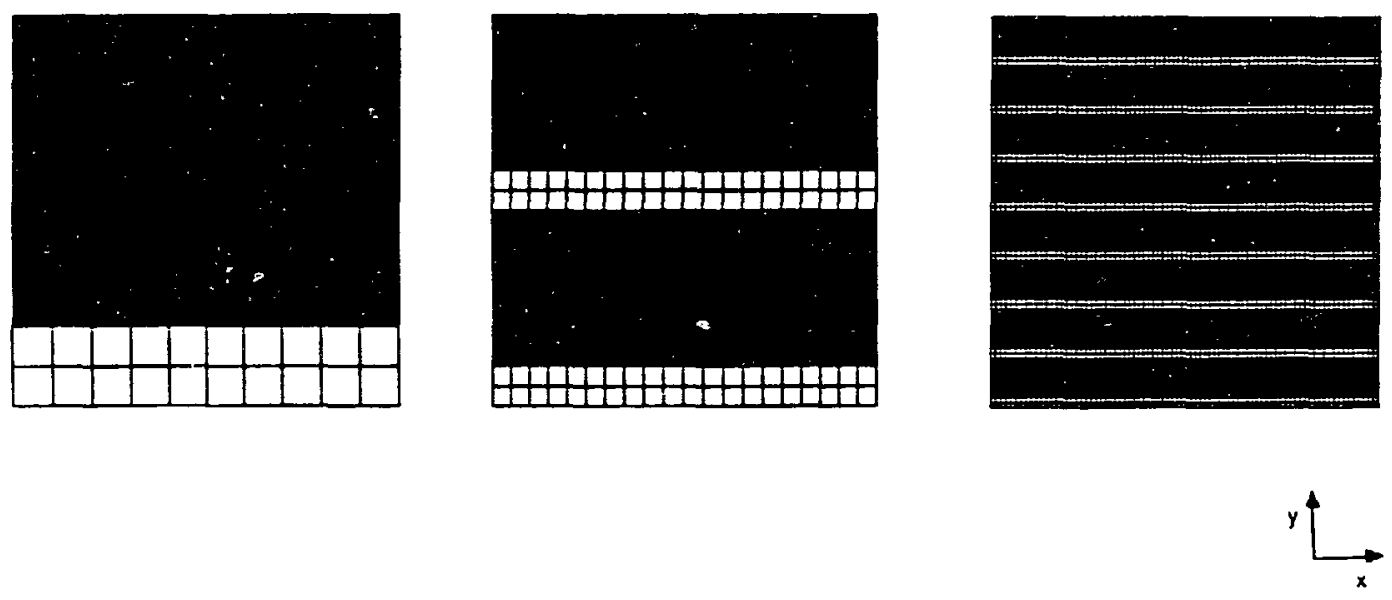

XBL 893-1075

Figure 5.4.4 Replication family of patterns for which NBREAK is invariant. 

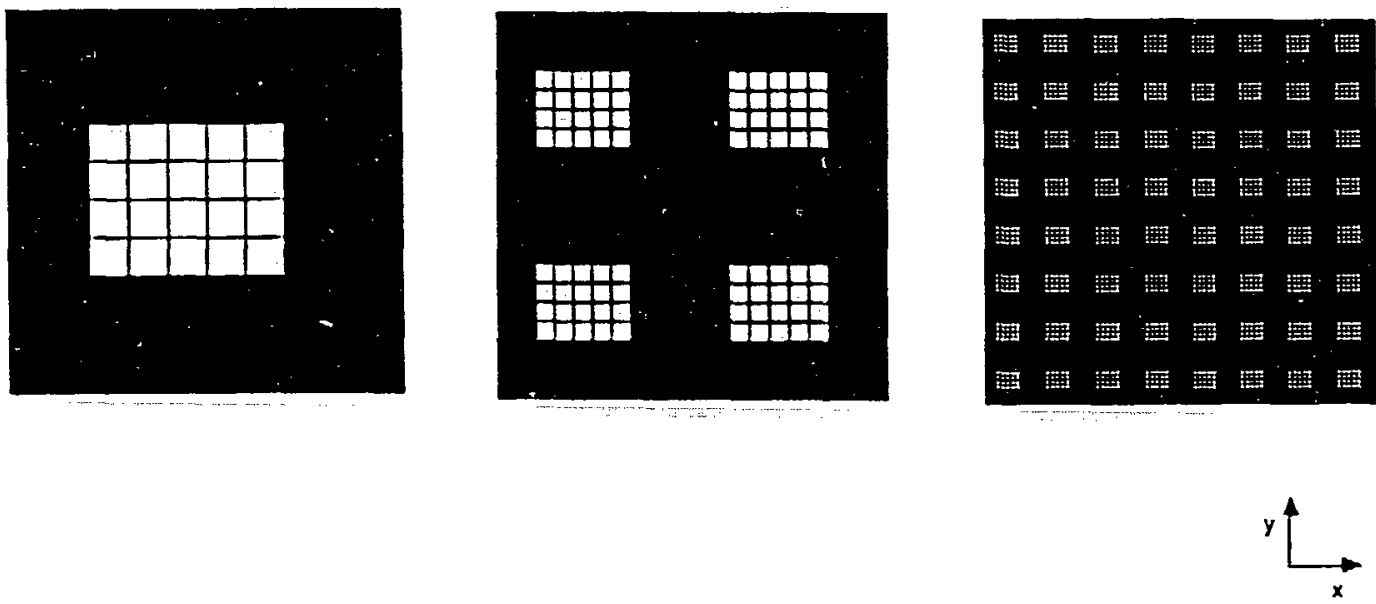

$$
\text { XBL 893-1046 }
$$

Figure 5.4.5 Replication family for which NBREAK is not invariant. 


\section{Simulated Patterns}

Table 5.4.1 and Table 5.4.2 list BREAK, RUN, NBREAK and $K_{\text {eq }}$ for the 10 by 10 and 30 by 30 pixel simulated patterns.

Table 5.4.1 Erosion statistics, equivalent permeability and predicted equivalent permeability for 10 by 10 pixel patterns with $80 \%$ voids.

\begin{tabular}{|c|c|c|c|c|c|}
\hline Pattern & $B R E A K$ & $R U N$ & NBREAK & $K_{\text {eq }}$ & $\hat{K}_{e q}$ \\
\hline $10 \mathrm{a}(\hat{y})$ & 3 & 4.211 & 0.713 & 0.564 & 0.618 \\
\hline $10 \mathrm{a}(\hat{x})$ & 3 & 5.000 & 0.600 & 0.457 & 0.575 \\
\hline $10 \mathrm{~b}(\hat{y})$ & 1 & 4.444 & 0.225 & 0.356 & 0.474 \\
\hline $10 \mathrm{~b}(\hat{x})$ & 4 & 4.444 & 0.900 & 0.665 & 0.603 \\
\hline $10 c(\hat{y})$ & 0 & 6.154 & 0.000 & 0.000 & \\
\hline $10 c(\hat{x})$ & 6 & 5.714 & 1.050 & 0.670 & 0.646 \\
\hline $10 d(\hat{y})$ & 3 & 8.000 & 0.375 & 0.465 & 0.489 \\
\hline $10 d(\bar{x})$ & 3 & 5.333 & 0.563 & 0.504 & 0.560 \\
\hline $10 \mathrm{e}(\hat{y})$ & 2 & 3.810 & 0.525 & 0.584 & 0.496 \\
\hline $10 e(\hat{x})$ & 2 & 5.000 & 0.400 & 0.324 & 0.460 \\
\hline $10 f(\hat{y})$ & 4 & 7.273 & 0.550 & 0.605 & 0.503 \\
\hline $10 f(\hat{x})$ & 5 & 6.154 & 0.813 & 0.624 & 0.625 \\
\hline $10 \mathrm{~g}(\hat{y})$ & 0 & 8.889 & 0.000 & 0.000 & \\
\hline $\log (\hat{x})$ & 7 & 8.000 & 0.875 & 0.776 & 0.632 \\
\hline $10 \mathrm{~h}(\hat{\mathrm{y}})$ & 1 & 8.000 & 0.125 & 0.343 & 0.417 \\
\hline $10 \mathrm{~h}(\hat{x})$ & 7 & 8.000 & 0.875 & 0.757 & 0.632 \\
\hline $10 \mathrm{i}(\hat{y})$ & 3 & 4.444 & 0.675 & 0.622 & 0.603 \\
\hline $10 \mathrm{i}(\hat{x})$ & 4 & 5.000 & 0.800 & 0.414 & 0.575 \\
\hline $10 \mathrm{j}(\hat{y})$ & 7 & 7.273 & 0.963 & 0.727 & 0.661 \\
\hline $10 \mathrm{j}(\hat{x})$ & 2 & 6.667 & 0.300 & 0.376 & 0.432 \\
\hline
\end{tabular}


Table 5.4.2. Erosion statistics, equivalent permeability and predicted equivalent permeability for 30 by 30 pixel patterns with $80 \%$ voids.

\begin{tabular}{|c|c|c|c|c|c|}
\hline Pattern & $B R E A K$ & $R U N$ & NBREAK & $K_{e q}$ & $\hat{K}_{e q}$ \\
\hline 30a $(\hat{y})$ & 6 & 9.012 & 0.666 & 0.580 & 0.536 \\
\hline $30 a(\hat{x})$ & 5 & 10.603 & 0.472 & 0.567 & 0.507 \\
\hline $30 b(\hat{y})$ & 6 & 9.627 & 0.623 & 0.427 & 0.524 \\
\hline $30 \mathrm{~b}(\hat{x})$ & 7 & 9.256 & 0.756 & 0.518 & 0.593 \\
\hline $30 c(\hat{y})$ & 5 & 9.089 & 0.550 & 0.510 & 0.534 \\
\hline $30 c(\hat{x})$ & 10 & 10.716 & 0.933 & 0.507 & 0.613 \\
\hline $30 \mathrm{~d}(\hat{y})$ & 3 & 10.894 & 0.275 & 0.405 & 0.450 \\
\hline $30 \mathrm{~d}(\hat{x})$ & 6 & 9.849 & 0.609 & 0.486 & 0.520 \\
\hline $30 \mathrm{e}(\hat{y})$ & 11 & 11.852 & 0.928 & 0.606 & 0.636 \\
\hline $30 \mathrm{e}(\bar{x})$ & 6 & 10.329 & 0.581 & 0.535 & 0.512 \\
\hline 30f $(\hat{y})$ & 8 & 9.244 & 0.865 & 0.512 & 0.593 \\
\hline $30 \mathrm{f}(\hat{x})$ & 4 & 9.244 & 0.433 & 0.496 & 0.469 \\
\hline $30 \mathrm{~g}(\hat{y})$ & 3 & 9.114 & 0.329 & 0.247 & 0.471 \\
\hline $30 \mathrm{~g}(\hat{x})$ & 4 & 8.372 & 0.478 & 0.486 & 0.482 \\
\hline $30 \mathrm{~h}(\bar{y})$ & 12 & 9.986 & 1.202 & 0.587 & 0.690 \\
\hline 30h $(\hat{x})$ & 2 & 11.234 & 0.178 & 0.431 & 0.396 \\
\hline 30i $(\hat{y})$ & 4 & 7.783 & 0.514 & 0.553 & 0.493 \\
\hline $30 \mathrm{i}(\hat{x})$ & 2 & 7.783 & 0.257 & 0.422 & 0.419 \\
\hline $30 \mathrm{j}(\hat{y})$ & 4 & 10.183 & 0.393 & 0.566 & 0.458 \\
\hline $30 \mathrm{j}(\hat{x})$ & 8 & 9.904 & 0.808 & 0.425 & 0.577 \\
\hline 30k $(\hat{y})$ & 3 & 11.581 & 0.259 & 0.419 & 0.444 \\
\hline 30k $(\hat{x})$ & 12 & 10.257 & 1.170 & 0.634 & 0.681 \\
\hline $301(\hat{y})$ & 3 & 7.793 & 0.385 & 0.439 & 0.492 \\
\hline $301(\hat{x})$ & 3 & 7.793 & 0.385 & 0.525 & 0.492 \\
\hline $30 \mathrm{~m}(\hat{y})$ & 6 & 8.287 & 0.724 & 0.504 & 0.553 \\
\hline $30 \mathrm{~m}(\hat{x})$ & 4 & 8.482 & 0.472 & 0.561 & 0.480 \\
\hline
\end{tabular}

Figures 5.4.6 shows the correlation between equivalent permeability and the erosion statistic.

\section{Prediction Model}

Since Figure 5.4.6 shows a linear trend between equivalent permeability and NBREAK for patterns with positive equivalent permeability, a model linear in NBREAK was fit to data from the 10 by 10 pixel and 30 by 30 pixel patterns. The model is

$$
K_{\text {oq }}=\alpha+\beta N B R E A K
$$

where $\alpha$ and $\beta$ are to determined. The best fit values of $\alpha$ and $\beta$ minimize 
Erosion Statistic Model

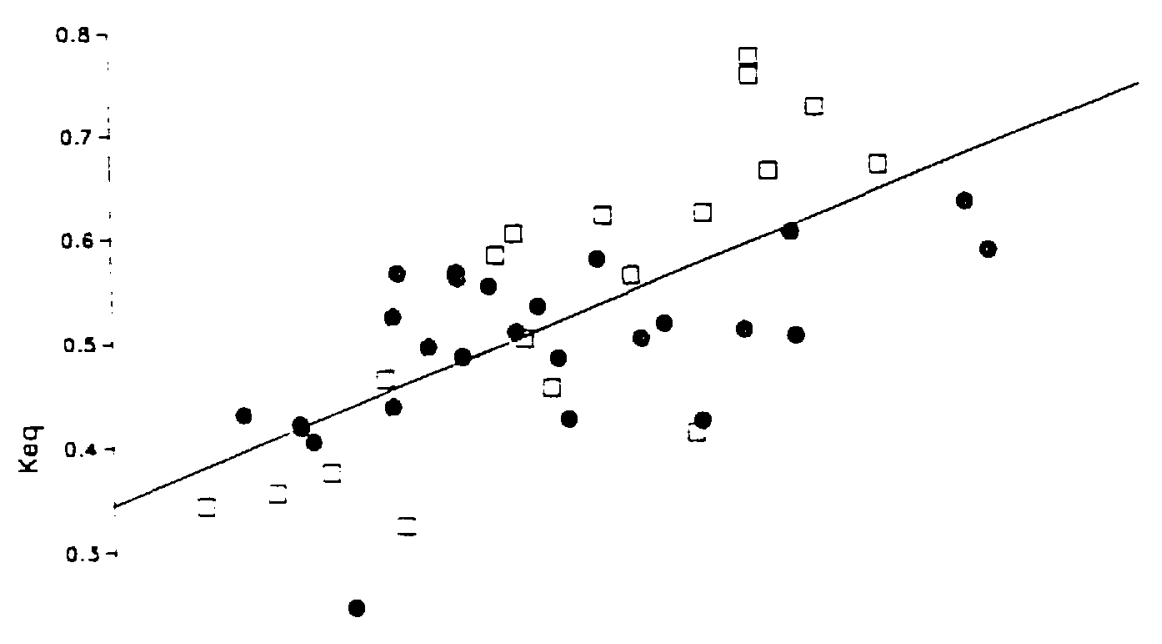

$0.2+$

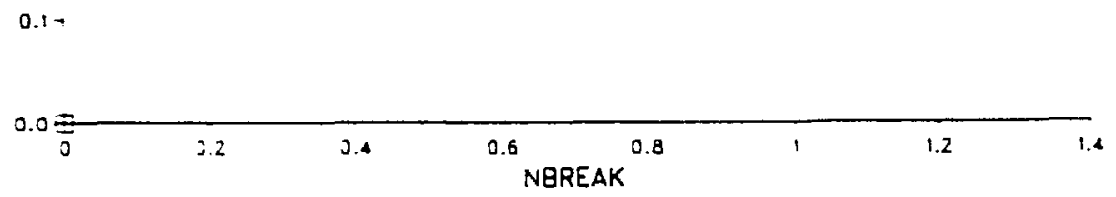

Figure 5.4.6 Equivalent permeability versus NBREAK for 10 by 10 pixel and 30 by 30 pixel simulated patterns with $80 \%$ voids. Predicted equivalent permeability according to NBREAK model is plotted as a line. 


$$
R \hat{S E}=\left[\frac{1}{M-2} \sum_{i=1}^{M}\left(K_{e q}-\hat{K}_{e q}(\hat{\alpha}, \hat{\beta})_{i}\right)^{2}\right]^{\frac{1}{2}}
$$

where $M$ is the number of data points in the fit. For the 10 by 10 pixel data set, $M=18$. For the 30 by 30 pixel data set, $M=26$. Since the $K_{\text {eq }}=0$ data points do not follow the trend defined by the other data points, they were excluded from the regression. However, patterns with zero equivalent permeability are trivial cases because they have NBREAK $=0$. Table 5.4.3 shows the fitted values of the model parameters. Figure 5.4.6 plots predicted equivalent permeability along with actual equivalent permeability. To predict equivalent permeability, the 44 pattern values of the fitted parameters were used. Tables 5.4.1 and 5.4.2 list predicted equivalent permeability beside actual equivalent permeability for each pattern. Note that the intercepts are significantly above zero. Hence, the prediction model inconsistently predicts a nonzero equivalent permeability for patterns with NBREAK $=0$. Thus, the model is not intended for patterns with equivalent permeabilities which are close to zero. Instead, the model is intended for patterns with equivalent permeabilities which fall in the range of those computed for the simulated patterns.

Table 5.4.3. Fitted parameters for Erosion statistic prediction model.

\begin{tabular}{|ccccc|}
\hline scale & $M$ & $\alpha($ s.e. $)$ & $\beta($ s.e. $)$ & $R \hat{S E}$ \\
\hline \hline 10 & 18 & $0.261(0.052)$ & $0.453(0.077)$ & 0.085 \\
30 & 26 & $0.399(0.032)$ & $0.169(0.050)$ & 0.069 \\
both & 44 & $0.345(0.032)$ & $0.287(0.048)$ & 0.085 \\
\hline
\end{tabular}

\section{Scale Effect}

Table 5.4.3 shows that the fitted parameters are sensitive to scale. The test statistics to determine if the fitted intercept $(\hat{\alpha})$ and slope $(\hat{\beta})$ significantly differ at the two scales are

$$
\frac{\left|\hat{\alpha}_{10}-\hat{\alpha}_{30}\right|}{\sqrt{\sigma_{a_{10}}{ }^{2}+\sigma_{a_{30}}^{2}}}=2.26
$$


and

$$
\frac{\left|\beta_{10}-\beta_{30}\right|}{\sqrt{\sigma_{\beta_{10}}{ }^{2}+\sigma_{\beta_{30}}{ }^{2}}}=3.09 .
$$

The difference between the fitted slopes is very significant; we would reject a hypothesis that that slopes are the same with confidence over $99 \%$ using standard hypothesis testing (Mood et al., 1974). Similarly, the hypothesis that the intercepts are the same is rejected at $96 \%$ confidence. Perhaps the scale effect reflects the sensitivity of BREAK and RUN to boundary effects that occur for patterns with only a few contact area islands. Further, the fitted parameters may be different for simulated patterns with scales in between the 10 by 10 and 30 by 30 pixel scales studied. The scale dependence of the fitted parameters casts doubt on the utility of using NBREAK, alone, for predicting the equivalent permeability of other kinds of patterns.

\section{Summary}

A model which predicts equivalent permeability in terms of NBREAK was fit to data from the simulated patterns. However, the fitted parameters depended on scale. This suggests that NBREAK, alone, is not a reliable statistic to predict flow with. Next, NBREAK will be included in a multivariate model along with NCP, NSIGR and NSIGC to predict the equivalent permeability of the 10 by 10 and 30 by 30 pixel simulated patterns. 


\subsection{A Multivariate Modei}

\section{Introduction and Summary}

In this section, equivalent permeability is predicted in terms of a linear combination of the four spatial statistics examined in Sections 5.2,5.3 and 5.4. Below, a multivariate model including all four spatial statistics is fit to the 10 by 10 pixel and 30 by 30 pixel patterns with $80 \%$ voids. Because the four statistic model fitted parameters are not well determined, a three statistic model is fit to the data from the simulated patterns. The three statistic model fitted parameters are well determined.

\section{Predictor Correlation}

Before fitting a multivariate model to data, it is important to determine that the the predictor variables are not obviously redundant. A redundant statistic carries the same information as one of the others, or a combination of the others. If a redundant statistic is included $i$, the regression, prediction is not improved and the fitted parameters will not be well determined. This is called colinearity. The most striking evidence for colinearity would be a linear relationship between one statistic and another. Figure 5.5.1 shows scatterplots of each spatial statistic versus each of the other spatial statistics for all 44 patterns. Because each plot has a lot of scatter, no two spatial statistics appear colinear. Next we fit a multivariate model involving all four spatial statistics to the data from the 10 by 10 and 30 by 30 pixel patterns.

\section{Model I}

The first multivariate model examined is

$$
\hat{K}_{\text {sq }}=0.8+\beta_{1}(N B R E A K-1)-\beta_{2} N C P-\beta_{3} N S I G C+\beta_{4}(N S I G R-1)
$$

where the $\beta$ 's are to be determined. This model is parsimonious because it is linear in the spatial statistics and predicts the correct equivalent permeability for the familiar pattem of voids and contact area shown in Figure 5.2.1. That is, for a pressure drop in 

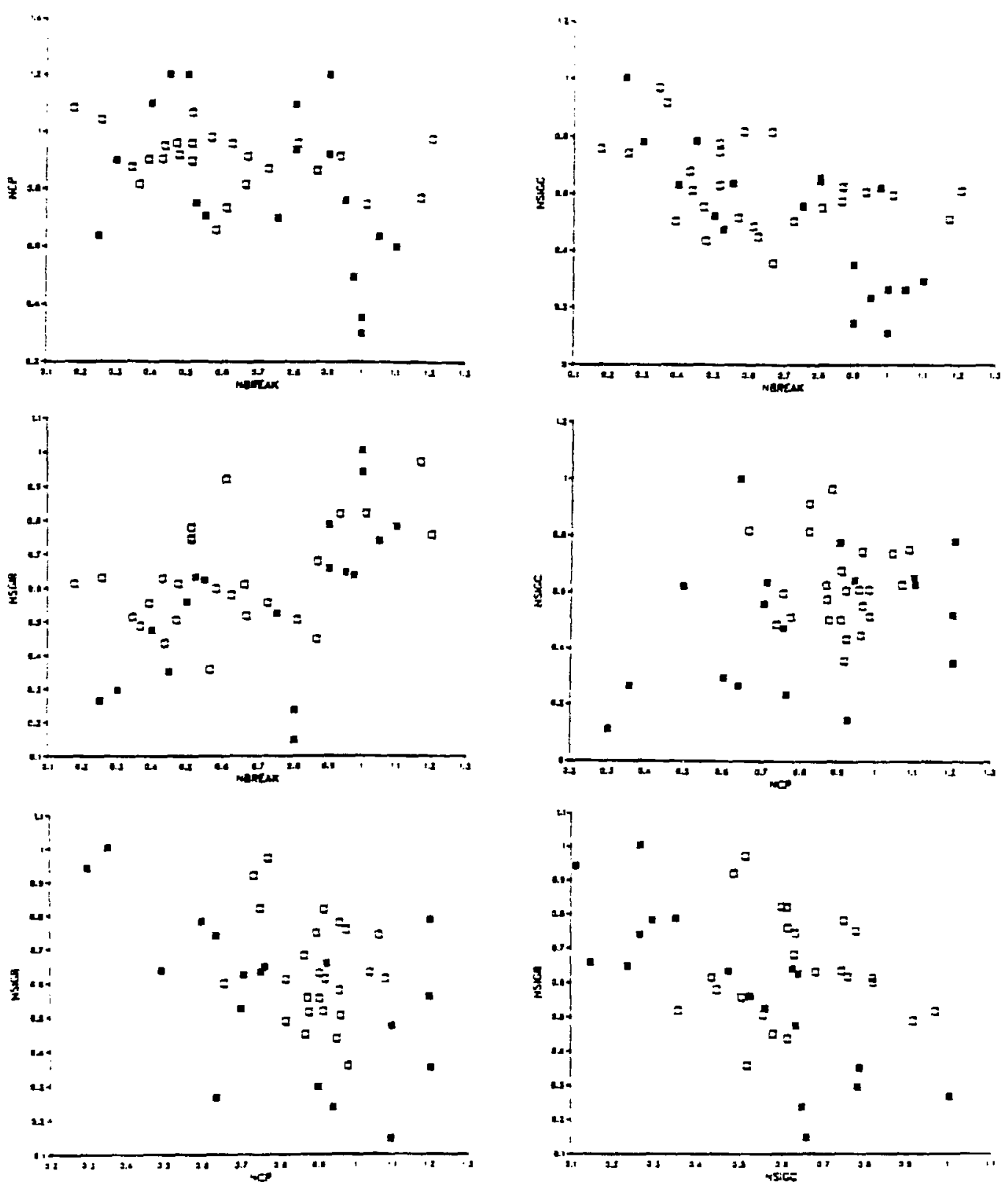

Legend

D 0 or c eatrems

- 30 or 3 sallerm

X9L $893-1077$

Figure 5.5.1 Scatterplots of spatial statistics of 10 by 10 pixel and 30 by 30 pixel simulated patterns with $80 \%$ voids. 
the $x$ direction, NSIGC and NCP are zero, NSIGR and NBREAK are one, and both $K_{\text {eq }}$ and $\hat{K}_{\text {eq }}$ are 0.80 .

The best fit values of the parameters $\beta_{1}, \beta_{2}, \beta_{3}$ and $\beta_{4}$ minimized

$$
R \hat{S E}=\left[\frac{1}{M-4} \sum_{i=1}^{M}\left(K_{e q}-\hat{K}_{a q}\right)^{2}\right]^{\frac{1}{2}}
$$

where $M$ is the number of data points in the fit. The model is fit to data from the 10 by 10 pixel pattems alone, the 30 by 30 pixel patterns alone and then both scales pooled together. As in sections 5.2, 5.3 and 5.4, the 10 by 10 patterns which had zero equivalent permeability were excluded from the fit.

Tables 5.5 .1 and 5.5 .2 show the statistics for the 10 by 10 pixel and 30 by 30 pixel patterns. 
Table 5.5.1. Spatial statistics, equivalent permeability and multivariate model prediction for the equivalent permeability of the 10 by 10 pixel patterns with $80 \%$ voids.

\begin{tabular}{|c|c|c|c|c|c|c|}
\hline & NBREAK & $N C P$ & NSIGC & NSIGR & $K_{\infty q}$ & $\hat{K}_{e q}$ \\
\hline $10 \mathrm{a}(\bar{y})$ & 0.713 & 0.760 & 0.236 & 0.647 & 0.564 & 0.591 \\
\hline $10 \mathrm{a}(\hat{x})$ & 0.600 & 0.939 & 0.647 & 0.236 & 0.457 & 0.420 \\
\hline $10 \mathrm{~b}(\hat{y})$ & 0.225 & 1.202 & 0.787 & 0.352 & 0.356 & 0.380 \\
\hline $10 \mathrm{~b}(\hat{x})$ & 0.900 & 1.202 & 0.352 & 0.787 & 0.665 & 0.535 \\
\hline $10 c(\hat{y})$ & 0.000 & 1.061 & 0.740 & 0.266 & 0.000 & \\
\hline $10 c(\hat{x})$ & 1.050 & 0.636 & 0.266 & 0.740 & 0.670 & 0.619 \\
\hline $10 \mathrm{~d}(\hat{y})$ & 0.375 & 1.200 & 0.524 & 0.559 & 0.465 & 0.464 \\
\hline $10 \mathrm{~d}(\hat{x})$ & 0.563 & 0.700 & 0.559 & 0.524 & 0.504 & 0.520 \\
\hline $10 \mathrm{e}(\hat{y})$ & 0.525 & 0.750 & 0.474 & 0.632 & 0.584 & 0.547 \\
\hline $10 \mathrm{e}(\hat{x})$ & 0.400 & 1.100 & 0.632 & 0.474 & 0.324 & 0.443 \\
\hline $10 f(\hat{y})$ & 0.550 & 0.707 & 0.638 & 0.624 & 0.605 & 0.522 \\
\hline $10 f(\hat{x})$ & 0.813 & 0.495 & 0.624 & 0.638 & 0.624 & 0.556 \\
\hline $10 \mathrm{~g}(\hat{y})$ & 0.000 & 1.000 & 0.942 & 0.112 & 0.000 & \\
\hline $10 \mathrm{~g}(\hat{x})$ & 0.875 & 0.300 & 0.112 & 0.942 & 0.776 & 0.729 \\
\hline $10 \mathrm{~h}(\hat{y})$ & 0.125 & 0.636 & 1.004 & 0.266 & 0.343 & 0.402 \\
\hline $10 \mathrm{~h}(\hat{x})$ & 0.875 & 0.354 & 0.266 & 1.004 & 0.757 & 0.705 \\
\hline $10 \mathrm{i}(\hat{y})$ & 0.675 & 0.924 & 0.147 & 0.658 & 0.622 & 0.587 \\
\hline $10 \mathrm{i}(\hat{x})$ & 0.800 & 1.097 & 0.658 & 0.147 & 0.414 & 0.381 \\
\hline $10 \mathrm{j}(\hat{y})$ & 0.963 & 0.600 & 0.296 & 0.783 & 0.727 & 0.626 \\
\hline $10 \mathrm{j}(\hat{x})$ & 0.300 & 0.900 & 0.783 & 0.296 & 0.376 & 0.412 \\
\hline
\end{tabular}


Table 5.5.2. Spatial statistics, equivalent permeability and multivariate model prediction for the equivalent permeability of the 30 by 30 pixel patterns with $80 \%$ voids.

\begin{tabular}{|c|c|c|c|c|c|c|}
\hline & NBREAK & $N C P$ & NSIGC & NSIGR & $\overline{K_{e q}}$ & $\overline{\hat{K}_{\text {eq }}}$ \\
\hline $30 \mathrm{a}(\hat{y})$ & 0.666 & 0.914 & 0.358 & 0.518 & 0.580 & 0.525 \\
\hline $30 \mathrm{a}(\hat{x})$ & 0.472 & 0.981 & 0.518 & 0.358 & 0.567 & 0.459 \\
\hline $30 \mathrm{~b}(\hat{y})$ & 0.623 & 0.958 & 0.449 & 0.578 & 0.427 & 0.514 \\
\hline $30 \mathrm{~b}(\hat{x})$ & 0.756 & 0.866 & 0.578 & 0.449 & 0.518 & 0.480 \\
\hline $30 c(\hat{y})$ & 0.560 & 0.816 & 0.819 & 0.610 & 0.510 & 0.472 \\
\hline $30 c(\hat{x})$ & 0.933 & 0.916 & 0.610 & 0.819 & 0.507 & 0.533 \\
\hline $30 \mathrm{~d}(\hat{y})$ & 0.275 & 0.816 & 0.919 & 0.487 & 0.405 & 0.432 \\
\hline $30 \mathrm{~d}(\hat{x})$ & 0.609 & 0.733 & 0.487 & 0.919 & 0.486 & 0.598 \\
\hline $30 \mathrm{e}(\hat{y})$ & 0.928 & 0.749 & 0.599 & 0.821 & 0.606 & 0.558 \\
\hline $30 \mathrm{e}(\bar{x})$ & 0.581 & 0.658 & 0.821 & 0.599 & .535 & 0.492 \\
\hline $30 f(\hat{y})$ & 0.865 & 0.864 & 0.629 & 0.680 & 0.512 & 0.512 \\
\hline $30 f(\hat{x})$ & 0.433 & 0.904 & 0.680 & 0.629 & 0.496 & 0.489 \\
\hline $30 \mathrm{~g}(\hat{y})$ & 0.329 & 0.951 & 0.613 & 0.436 & 0.247 & 0.460 \\
\hline $30 \mathrm{~g}(\hat{x})$ & 0.478 & 0.919 & 0.436 & 0.613 & 0.486 & 0.527 \\
\hline 30h $(\hat{y})$ & 1.202 & 0.978 & 0.613 & 0.758 & 0.587 & 0.513 \\
\hline $30 \mathrm{~h}(\hat{x})$ & 0.178 & 1.084 & 0.758 & 0.613 & 0.431 & 0.447 \\
\hline $30 \mathrm{i}(\hat{y})$ & 0.514 & 1.066 & 0.631 & 0.743 & 0.553 & 0.495 \\
\hline $30 \mathrm{i}(\bar{x})$ & 0.257 & 1.042 & 0.743 & 0.631 & 0.422 & 0.459 \\
\hline $30 \mathrm{j}(\hat{y})$ & 0.393 & 0.904 & 0.506 & 0.555 & 0.566 & 0.507 \\
\hline $30 \mathrm{j}(\hat{x})$ & 0.808 & 0.962 & 0.555 & 0.506 & 0.425 & 0.481 \\
\hline $30 k(\hat{y})$ & 0.259 & 0.876 & 0.970 & 0.514 & 0.419 & 0.420 \\
\hline $30 \mathrm{k}(\hat{x})$ & 1.170 & 0.770 & 0.514 & 0.970 & 0.634 & 0.597 \\
\hline $301(\hat{y})$ & 0.385 & 0.897 & 0.779 & 0.748 & 0.439 & 0.493 \\
\hline $301(\hat{x})$ & 0.385 & 0.959 & 0.748 & 0.779 & 0.525 & 0.495 \\
\hline $30 \mathrm{~m}(\hat{y})$ & 0.724 & 0.872 & 0.505 & 0.556 & 0.504 & 0.511 \\
\hline $30 \mathrm{~m}(\hat{x})$ & 0.472 & 0.962 & 0.556 & 0.505 & 0.561 & 0.480 \\
\hline
\end{tabular}


Table 5.5.3 shows fitted model parameters and estimated standard errors for the fitted parameters for the four statistic prediction model.

Table 5.5.3 Fitted parameters and root mean square prediction error for four statistic prediction model.

\begin{tabular}{|lcccccc|}
\hline Scale & $M$ & $\beta_{1}($ s.e $)$ & $\beta_{2}($ s.e $)$ & $\beta_{3}($ s.e. $)$ & $\beta_{4}($ s.e. $)$ & $R \hat{S} E$ \\
\hline \hline 10 & 18 & $0.230(0.063)$ & $0.044(0.032)$ & $-0.019(0.081)$ & $0.341(0.085)$ & 0.045 \\
30 & 26 & $0.096(0.063)$ & $0.154(0.069)$ & $0.135(0.087)$ & $0.102(0.106)$ & 0.073 \\
both & 44 & $0.116(0.046)$ & $0.096(0.035)$ & $0.159(0.054)$ & $0.163(0.062)$ & 0.063 \\
\hline
\end{tabular}

Table 5.5.3 shows that the fitted parameters were very different at each scale. Two of the parameters $\left(\beta_{2}\right.$ and $\left.\beta_{4}\right)$ varied by over a factor of three between the two scales. Also, one of the fitted parameters $\left(\beta_{3}\right)$ took the wrong sign at the 10 by by 10 scale. Further, the difference between the fitted value of $\beta_{2}$ using the 30 by 30 patterns and the fitted value of $\beta_{2}$ using all 44 patterns is about $50 \%$ of the 44 parameter value for $\hat{\beta}_{2}$. The same comment applies to $\beta_{4}$. However, because the standard errors are so large relative to the differences, the differences are not overwhelmingly statistically significant. The test statistics, i.e. the differences divided by estimated standard error for the differences, for testing the hypotheses that the model parameters are the same at the 10 by 10 and 30 by 30 scales are $1.50,1.45,1.30$ and 1.76 for $\beta_{1}, \beta_{2}, \beta_{3}$, and $\beta_{4}$ respectively. By the same reasoning as presented in Section 5.3, the null hypotheses that the parameters are the same at each scale would be rejected at confidence levels of $86 \%, 85 \%, 80 \%$ and $92 \%$.

Although the statistical tests do not decisively rule out that the possibility that the "true" value of the model parameters are scale independent, there is no denying that the fitted parameters were sensitive to scale. Hence, we next consider a three statistic model in hopes of getting a better determined model. Before moving on, we remark on the consistency caveat. A model just like Model 1, but with an an adjustable intercept, was fit to data from the 44 patterns. The fitted value for the intercept 
was $0.860(0.046)$ and $R \hat{S E}$ was 0.062 . Since $R \hat{S E}$ is about the same and the difference between 0.860 and 0.800 is only 1.3 standard errors, setting the intercept to 0.80 is reasonable.

Model 2

To get a better determined model, we leave out NBREAK and form Model 2 which is

$$
\hat{K}_{e q}=0.80-\beta_{1} N C P-\beta_{2} N S I G C+\beta_{3}(N S I G R-1)
$$

Since the fitted parameters in the NCP prediction model presented in Section 5.2 and the fitted parameters in the NSIGC and NSIGR model presented in Section 5.3 were less sensitive to scale than the fitted parameters in the NBREAK model in Section 5.4, NBREAK was left out of the model. The best fit value of the parameters minimized

$$
R \hat{S} E=\left[\frac{1}{M-3} \sum_{i=1}^{M}\left(K_{a q}-\hat{K}_{e q}\right)^{2}\right]^{\frac{1}{2}}
$$

Table 5.5.4 shows that the parameters were better determined than the Model 1 parameters.

Table 5.5.4. Fitted parameters and root mean square prediction error for three statistic prediction model.

\begin{tabular}{|cccccc|}
\hline Scale & $M$ & $\beta_{1}($ s.e. & $\beta_{2}($ s.e. $)$ & $\beta_{3}$ (s.e.) & $R \hat{S E}$ \\
\hline \hline 10 & 18 & $0.053(0.043)$ & $0.142(0.093)$ & $0.343(0.114)$ & 0.060 \\
30 & 26 & $0.110(0.047)$ & $0.200(0.063)$ & $0.179(0.085)$ & 0.072 \\
both & 44 & $0.091(0.037)$ & $0.215(0.053)$ & $0.212(0.065)$ & 0.067 \\
\hline
\end{tabular}

The fitted parameters corresponding to fitting the model to the 30 by 30 pixel patterns alone are only slightly different from the fitted parameters from fitting the model to data from all 44 patterns. Further, if the 44 pattern fitted parameter values are used to estimate the equivalent permeability of the 10 by 10 pixel pattems, $R \hat{S} E$ is 0.064 . Thus, the 44 pattern fitted values predict the equivalent permeability of the 10 by 10 
pixel patterns almost as well as the 10 by 10 pixel fitted values do. Hence, the 44 pattern fitted values are good for both scales.

Predicted equivalent permeability, i.e.,

$$
\hat{K}_{\text {eq }}=0.8-0.091 N C P-0.215 N S I G C+.212(N S I G R-1) \text {, }
$$

is listed for each pattem in Tables 5.5.1 and 5.5.2. Further, $\hat{K}_{e q}$ is plotted versus $K_{e q}$ in Figure 5.5.2.

\section{Consistency}

To determine if a inconsistent model fits the data better than the consistent model considered, we fit a model linear in $N C P, N S I G C$ and $N S I G R-1$ with an adjustable intercept to the data from the 44 patterns. The new RSE (0.065) was almost the same as the consistent model RSE (0.067). Since the improvement is slight, setting the intercept to 0.80 is reasonable.

To illustrate how including three spatial statistics in a multivariate model improved prediction, Table 5.5.5 lists the RSE for the univariate models from Sections 5.2, 5.3 and 5.4 as well as the multivariate model developed in this section.

Table 5.5.5. Comparison of $R \hat{S} E$ for prediction models for patterns with $80 \%$ voids.

\begin{tabular}{|cc|}
\hline Model & $R \hat{S} E$ \\
\hline \hline $0.8-0.327$ NCP & 0.095 \\
$0.8-0.296$ NSIGC +0.277 (NSIGR -1$)$ & 0.071 \\
$0.345+0.287$ NBREAK & 0.085 \\
$0.8-0.091$ NCP -0.215 NSIC $+0.212($ NSIGR -1$)$ & 0.067 \\
\hline
\end{tabular}

\section{Summary}

A model to predict the equivalent permeability of the 10 by 10 and 30 by 30 pixel simulated patterns in terms of all four spatial statistics was fit. However, the 
Three statistic model

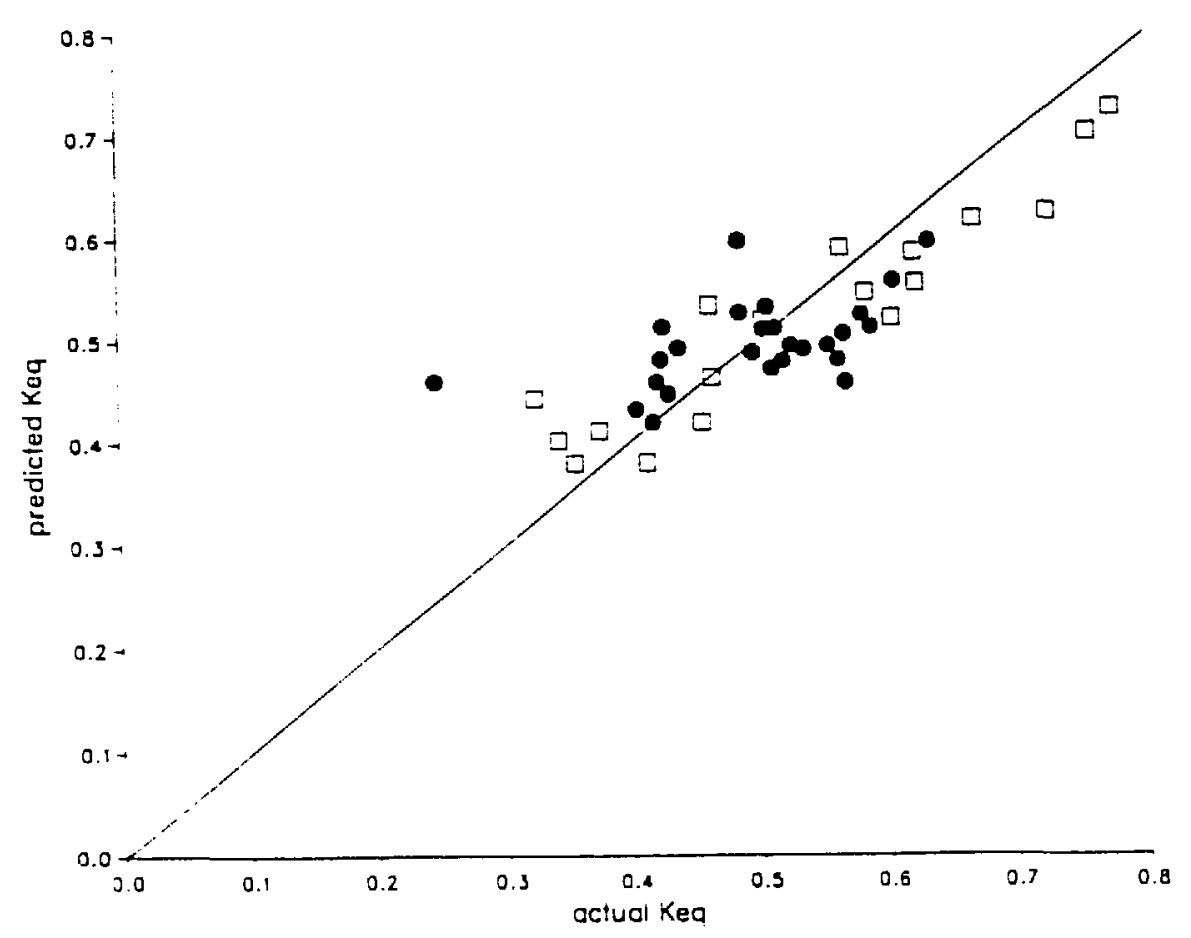

Figure 5.5.2 Predicted equivalent permeability according to three statistic (NCP, NSIGC and NSIGR ) model versus actual equivalent permeability for 10 by 10 pixel and 30 by 30 pixel simulated patterns with $80 \%$ voids. 
fitted parameters were sensitive to scale and not well determined. However, a three statistic model

$$
\hat{K}_{\text {eq }}=0.8-\beta_{1} N C P-\beta_{2} N S I G C+\beta_{3}(N S I G R-1)
$$

had better determined parameters. When the model was fit to data from the 30 by 30 pixel patterns, and then to both the 10 by 10 pixel and 30 by 30 pixel patterns, the fitted parameters were close. Further, the fitted parameter values from the pooled fit predicted the equivalent permeability of the 10 by 10 patterns almost as well as using the 10 by 10 pixel pattern fitted values. This suggests that the 44 pattern fitted model may work for other scales too. In the next section, models for predicting the equivalent permeability of patterns with other void fractions are presented. 


\subsection{Extension to Other Void Fractions}

\section{Introduction and Summary}

Chapter 5 presented a model to predict the equivalent permeability of a pattern with 0.80 void fraction in terms of spatial statistics computed from the arrangement of voids and contact areas within the pattem. This Chapter presents two parsimonious extensions of the 0.80 void fraction model. In 6.1, two candidate models are fit to data from 30 by 30 and 64 by 64 pixel simulated pattems with void fractions between 0.60 and 0.95 . One extended model (Model A) predicts equivalent permeability in terms of three spatial statistics (NCP, NSIGC, NSIGR) considered in Chapter 5. The other (Model B) predicts equivalent permeability in terms of one of the spatial statistics $(N C P)$. All of these patterns of voids and contact areas result from clipping a correlated Gaussian process as described in Chapter 3. In Section 6.2, the equivalent permeabilities of the simulated and observed 128 by 128 pixel pattems are predicted with the extended models. Both models are also applied to patterns simulated by other algorithms. To get one kind of different pattern, the shape of the moving average filter in the Chapter 3 model is modified. Other kinds of patterns are simulated using fractal ideas (Mandelbrot, 1983). For the patterns simulated using fractal ideas, Model B did a much better job of predicting flow than did Model A. Although Model B did well in predicting the equivalent permeability of most all of the patterns studied, there was one pattern for which it too failed. This pattern was so inhomogeneous, that flow was nearly blocked off. This limitation is discussed in more detail later. First, we present each candidate model. 


\subsection{Extended Models}

Recall that in Section 5.5, the equivalent permeability of a pattern with $80 \%$ voids was predicted as

$$
\hat{K}_{\text {eq }}=0.80-0.091 N C P-0.215 N S I G C+0.212(N S I G R-1)
$$

where NCP, NSIGC and NSIGR are spatial statistics computed from each pattern. The obvious extension of the above to other void fractions $p$ is

$$
\hat{K}_{e q}=p-\alpha_{1}(p) N C P-\alpha_{2}(p) N S I G C+\alpha_{3}(p)(N S I G R-1) .
$$

In principle, the coefficients can be empirically determined by repeating the kind of analysis that was done to get the 0.80 void fraction model. To do this, many more patterns would have to be simulated at each void fraction of interest. Rather than finding three new coefficients for each new void fraction of interest, simple linear extensions are offered. These extensions are parsimonious; only one new parameter has to be estimated. Further, at $p=0.80$, the extended models predict the same equivalent permeability that Eq. 6.1.1 does.

The first guess for an extended model, called Model A, is

$$
\hat{K}_{a q}{ }^{A}=p-[0.091 N C P+0.215 N S I G C-0.212(N S I G R-1)]\left[1+\beta_{A}(p-0.80)\right]
$$

where $\beta_{A}$ is to be determined. Although Model $A$ incorporates all three spatial statistics, it assumes a rather simplistic form for the $p$ dependence of the coefficients; the relative ratios of the coefficients do not depend on $p$. To get away from such a restrictive assumption, the simplest thing to do is to consider an extended model with only one spatial statistic. We extended the model from Section 5.2

$$
\hat{K}_{\text {eq }}=0.80-0.327 N C P
$$

as follows

$$
\hat{K}_{e q}{ }^{B}=p-0.327 N C P\left[1+\beta_{B}(p-0.8)\right]
$$


where $\beta_{B}$ is to be determined. We chose the $N C P$ model because its fitted parameter was not sensitive to scale effects like the NBREAK model fitted parameter was. We could also have extended a model to predict equivalent permeability in terms of NSIGC alone, but did not. A comparison between the NCP extended model and a NSIGC extended model would be interesting to make. This alternative extension (Eq. 6.1.5) is called Model B. The remaining part of this Chapter will deal with determining the empirical model parameters, $\beta_{A}$ and $\beta_{B}$, and comparing how well these two models predict equivalent permeability for a variety of patterns.

\section{Determination of model parameters}

Models $\mathrm{A}$ and $\mathrm{B}$ are fit to data from eight 30 by 30 pixel patterns (Figures 6.1.1 and 6.1.2) and four 64 by 64 pixel patterns (6.1.3) with void fractions between 0.60 and 0.95 . For each of the 64 by 64 pixel patterns and for the 30 by 30 pixel patterns shown in Figure 6.1.2, equivalent permeability was computed for both pressure drop directions. For the four 30 by 30 pixel patterns shown in Figure 6.1.1, equivalent permeability was computed for a pressure drop in the $y$ direction. Thus, in all, twenty equivalent permeabilities were computed. The best fit values of $\beta_{A}$ and $\beta_{B}$ minimized the sum of the square of the prediction errors for these patterns. That is, $\hat{\beta}_{A}$ was chosen to minimize

$$
R \hat{S} E_{A}=\left[\frac{1}{19} \sum_{i=1}^{20}\left(K_{e q}-\hat{K}_{e q}{ }^{A}\left(\beta_{A}\right)\right)_{i}^{2}\right]^{\frac{1}{2}}
$$

where $K_{\iota q}$ and $\tilde{K}_{a q}{ }^{A}\left(\beta_{A}\right)$ denoted actual and Model A predicted equivalent permeability. Similarly, $\hat{\beta}_{B}$ was chosen to minimize

$$
R \hat{S} E_{B}=\left[\frac{1}{19} \sum_{i=1}^{20}\left(K_{e q}-\hat{K}_{a q}^{B}\left(\beta_{B}\right)\right)_{i}{ }^{2}\right]^{\frac{1}{2}}
$$

To get the 30 by 30 pixel patterns, shown in Figure 6.1.1, the same realization of a correlated Gaussian Process was clipped at different levels. This realization was generated using the same model which simulated the 0.80 void fraction patterns 

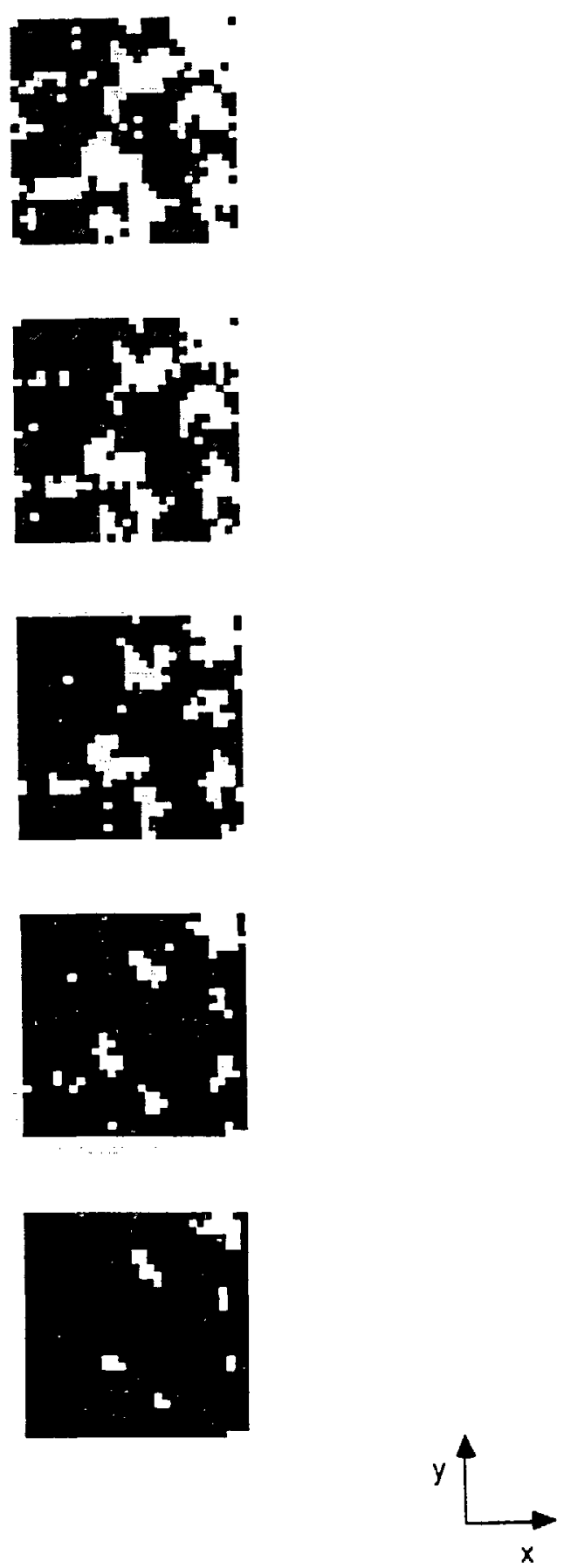

XBL $893-1079$

Figure 6.1.1 From the top, simulated patterns $30 \mathrm{~m}, 30 \mathrm{n}, 30 \mathrm{o}, 30 \mathrm{p}, 30 \mathrm{q}$. Void fractions are $0.60,0.70,0.80,0.90$ and 0.95 . Voids have constant aperture. 

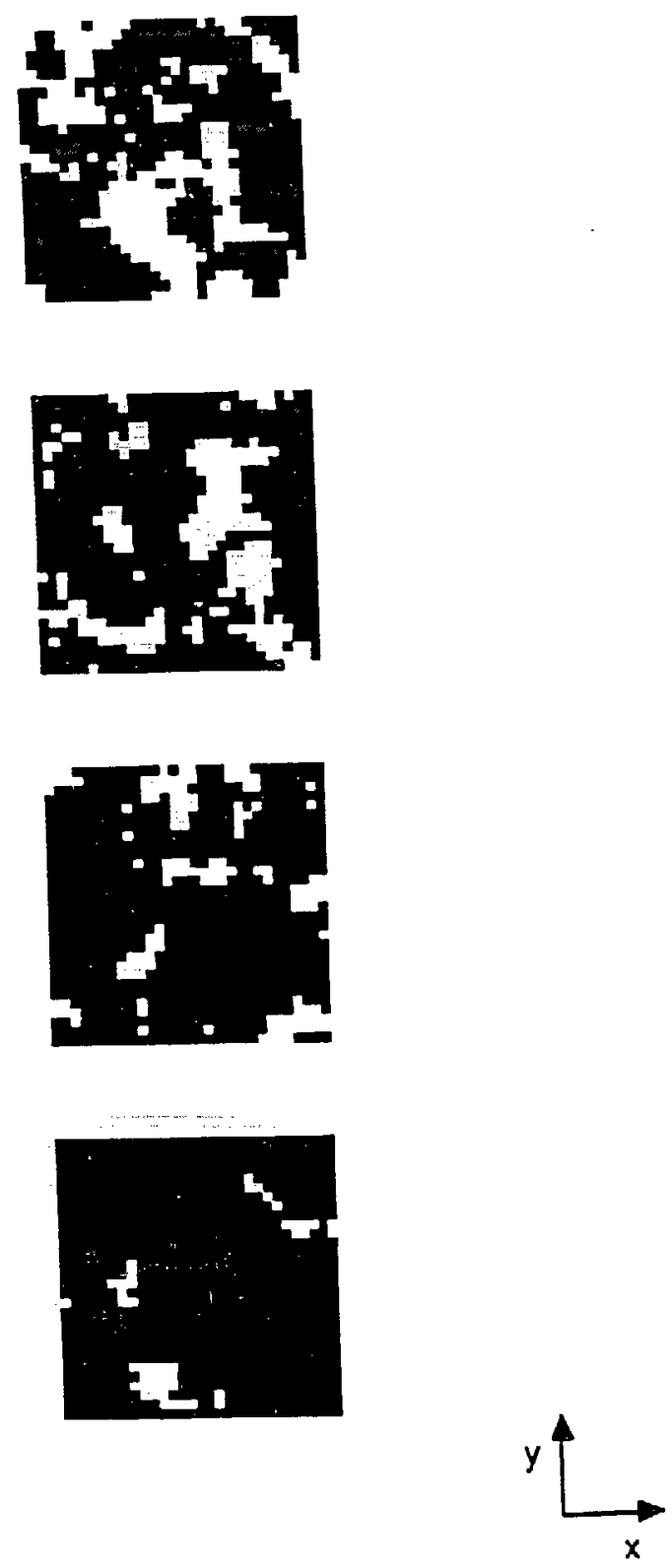

XBL 893-1080

Figure 6.1.2 From the top, simulated patterns $30 \mathrm{r}, 30 \mathrm{~s}, 30 \mathrm{t}$, and $30 \mathrm{u}$. Void fractions are $0.65,0.75,0.85$, and 0.95 . Voids have constant aperture. 

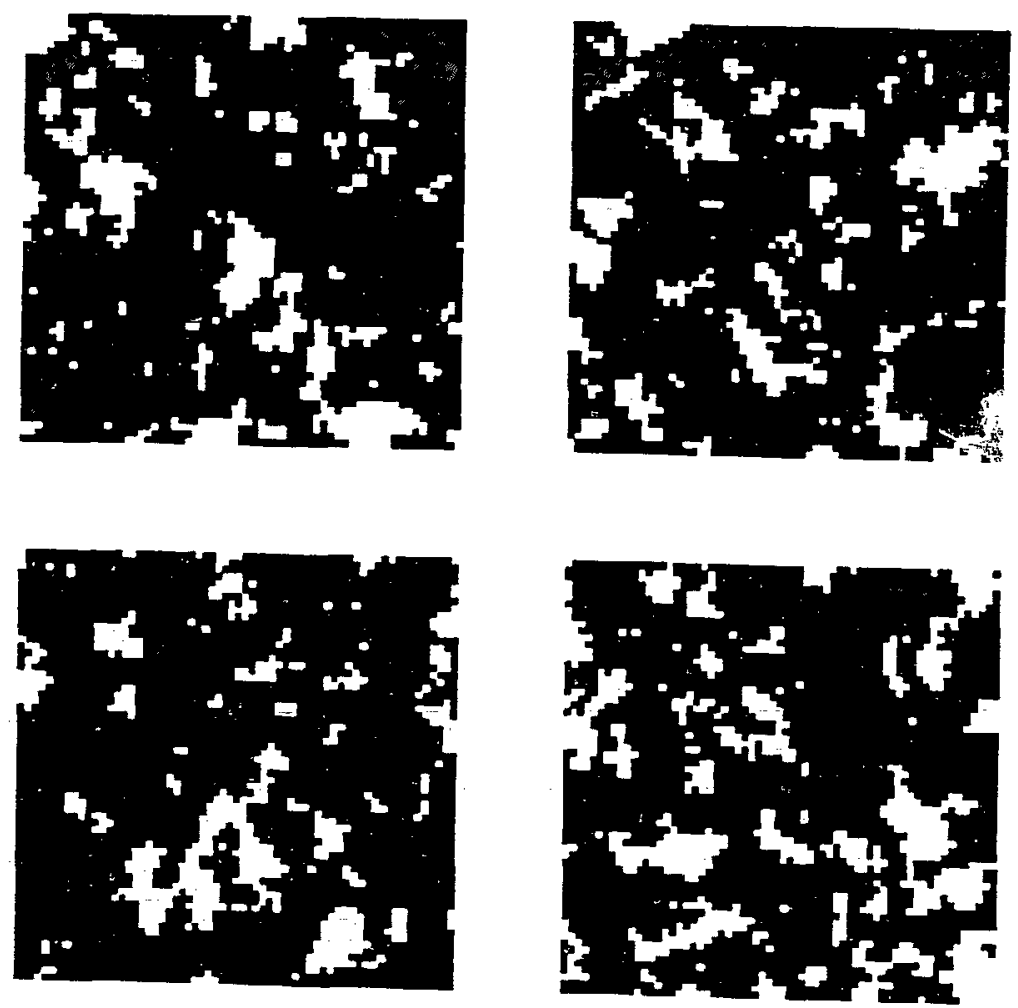

Figure 6.1.3 In clockwise order, from upper left, simulated patterns $64 a, 64 b, 64 c$ and $64 \mathrm{~d}$. Voids have constant aperture. 
discussed in Chapter 3. The moving average filter was the same; the weights dropped off exponentially where the damping factor( $(x)$ equaled 0.75 (inverse pixel lengths). To get the 30 by 30 pixel patterns shown in Figure 6.1.2, four different realizations from the same simulation model were clipped at different levels.

Equivalent permeability was determined using the finite-difference method described in Chapter 4. Table 6.1.1 shows how the equivalent permeability of pattern $30 \mathrm{n}$, computed for a pressure drop in the $y$ direction, depends on mesh size. Computed equivalent permeability is the same within a half percent, for meshes of size 90 or 90 or larger. Hence, a 150 by 150 mesh determines equivalent permeability to a precision of a percent or so. Since equivalent permeability varies among the new patterns by much more than this error, the numerical method is adequate. For all the 30 by 30 pixel patterns studied in this chapter, a 150 by 150 mesh was used to determine equivalent permeability. Hence, each pixel is assigned $5^{2}$ nodes.

Table 6.1.1. Equivalent permeability dependence on mesh size for pattern 30n (v).

\begin{tabular}{|ccc|}
\hline mesh & irerations & $K_{\text {eq }}$ \\
\hline \hline 30 by 30 & 8000 & 0.138 \\
60 by 60 & 6000 & 0.326 \\
90 by 90 & 4000 & 0.345 \\
120 by 120 & 2000 & 0.348 \\
150 by 150 & 2000 & 0.350 \\
\hline
\end{tabular}


Table 6.1.2 lists all the patterns and computed equivalent permeability using 150 by 150 meshs.

Table 6.1.2. Spatial statistics, predicted and actual equivalent permeability for $\mathbf{3 0}$ by 30 pixel patterns with variable void fractions.

\begin{tabular}{|c|c|c|c|c|c|c|c|}
\hline & $p$ & $N C P$ & NSIGC & NSIGR & $K_{e q}$ & $\hat{K}_{e q}{ }^{A}$ & $\hat{K}_{e q}^{B}$ \\
\hline $30 \mathrm{~m}(\hat{y})$ & 0.600 & 1.258 & 0.551 & 0.949 & 0.101 & 0.178 & 0.112 \\
\hline 30n $(\hat{y})$ & 0.700 & 1.036 & 0.509 & 0.985 & 0.350 & 0.417 & 0.330 \\
\hline $300(\hat{y})$ & 0.800 & 0.938 & 0.500 & 0.839 & 0.551 & 0.573 & 0.493 \\
\hline 30p $(\hat{y})$ & 0.900 & 0.600 & 0.418 & 0.746 & 0.756 & 0.775 & 0.722 \\
\hline $30 \mathrm{q}(\hat{y})$ & 0.950 & 0.424 & 0.434 & 0.522 & 0.868 & 0.845 & 0.831 \\
\hline $30 \pi(\hat{y})$ & 0.650 & 1.269 & 0.447 & 0.576 & 0.257 & 0.182 & 0.176 \\
\hline $30 \mathrm{r}(\hat{x})$ & 0.650 & 1.269 & 0.576 & 0.447 & 0.175 & 0.097 & 0.176 \\
\hline 30s $(\tilde{y})$ & 0.750 & 1.073 & 0.587 & 0.827 & 0.310 & 0.442 & 0.383 \\
\hline $30 \mathrm{~s}(\hat{x})$ & 0.750 & 1.066 & 0.827 & 0.587 & 0.298 & 0.321 & 0.385 \\
\hline $30 \mathrm{t}(\hat{y})$ & 0.850 & 0.753 & 0.678 & 0.480 & 0.627 & 0.585 & 0.615 \\
\hline $30 t(\hat{x})$ & 0.850 & 0.738 & 0.480 & 0.678 & 0.654 & 0.655 & 0.620 \\
\hline 30u $(\hat{y})$ & 0.950 & 0.533 & 0.487 & 0.431 & 0.854 & 0.827 & 0.800 \\
\hline $30 u(\hat{x})$ & 0.950 & 0.433 & 0.431 & 0.487 & 0.851 & 0.842 & 0.828 \\
\hline
\end{tabular}

To get the 64 by 64 pixels patterns with variable void fractions, pattern $128 \mathrm{~b}$ from Figure 3.2 of Chapter 3, was partioned into four quadrants. Figure 6.1.3 shows the four 64 by 64 pixel patterns $(64 a, 64 b, 64 c, 64 d)$ which result. Again, using the methods described in Chapter 4, equivalent permeability was determined. Table 6.1.3 shows how equivalent permeability depended on mesh size for pattern $64_{4}$. The pressure drop was in the $x$ direction.

Table 6.1.3. Equivalent permeability dependence on mesh size for pattern $64 a$ $(\hat{x})$.

\begin{tabular}{|ccc|}
\hline mesh & iterations & $K_{\text {eq }}$ \\
\hline \hline 64 by 64 & 4000 & 0.448 \\
128 by 128 & 2000 & 0.440 \\
192 by 192 & 2000 & 0.456 \\
256 by 256 & 800 & 0.456 \\
\hline
\end{tabular}


Based on this example, the numerical method determines equivalent permeability to a precision of a percent or so. Since the variation of equivalent permeability among the 64 by 64 pattems is far greater than a percent or two, this precision is adequate. Table 6.1.4 lists the statistics and equivalent permeabilities, determined with a 256 by 256 mesh, for all the 64 by 64 pixel patterns.

Table 6.1.4. Spatial statistics, predicted equivalent permeability and actual equivalent permeability for 64 by 64 pixel patterns.

\begin{tabular}{|cccccccc|}
\hline & $p$ & $N C P$ & NSIGC & NSIGR & $K_{\text {eq }}$ & $\hat{K}_{\text {eq }}{ }^{{ }^{4}}$ & $\hat{K}_{\text {eq }}{ }^{B}$ \\
\hline \hline 64a $(\hat{y})$ & 0.826 & 0.781 & 0.636 & 0.727 & 0.559 & 0.586 & 0.577 \\
64a $(\hat{x})$ & 0.826 & 0.869 & 0.727 & 0.636 & 0.456 & 0.543 & 0.549 \\
64b (y) & 0.818 & 0.905 & 0.506 & 0.574 & 0.500 & 0.555 & 0.527 \\
64b $(\hat{x})$ & 0.818 & 0.883 & 0.574 & 0.506 & 0.528 & 0.530 & 0.534 \\
64c $(\hat{y})$ & 0.854 & 0.738 & 0.592 & 0.724 & 0.615 & 0.650 & 0.624 \\
64c $(\hat{x})$ & 0.854 & 0.801 & 0.724 & 0.592 & 0.553 & 0.600 & 0.605 \\
64d $(\hat{y})$ & 0.768 & 1.093 & 0.654 & 0.740 & 0.410 & 0.439 & 0.403 \\
64d $(\hat{x})$ & 0.768 & 1.105 & 0.740 & 0.654 & 0.399 & 0.396 & 0.396 \\
\hline
\end{tabular}

Table 6.1.5 shows how well each model fit the data.

Table 6.1.5. Fitted parameters and root mean square prediction error for extended models.

\begin{tabular}{|cccc|}
\hline$\hat{\beta}_{A}($ s.e. $)$ & $R \hat{S} E_{A}$ & $\hat{\beta}_{B}($ s.e. $)$ & $R \hat{S} E_{B}$ \\
\hline \hline$-3.67(0.47)$ & 0.056 & $-0.938(0.328)$ & 0.046 \\
\hline
\end{tabular}

Since Model $\mathrm{B}$ is more parsimonious than Model $\mathrm{A}$, and $R \hat{S} E_{B}$ is less than $R \hat{S} E_{A}$, Model B seems preferable to Model A. However, since the $R \hat{S E}$ 's are close, we will compare how each model does for a variety of patterns. Figures 6.1.4 and 6.1.5 plor predicted versus actual equivalent permeability for Model A and B for the 30 by 30 pixel and 64 by 64 pixel patterns that were used to determine the model parameters. 


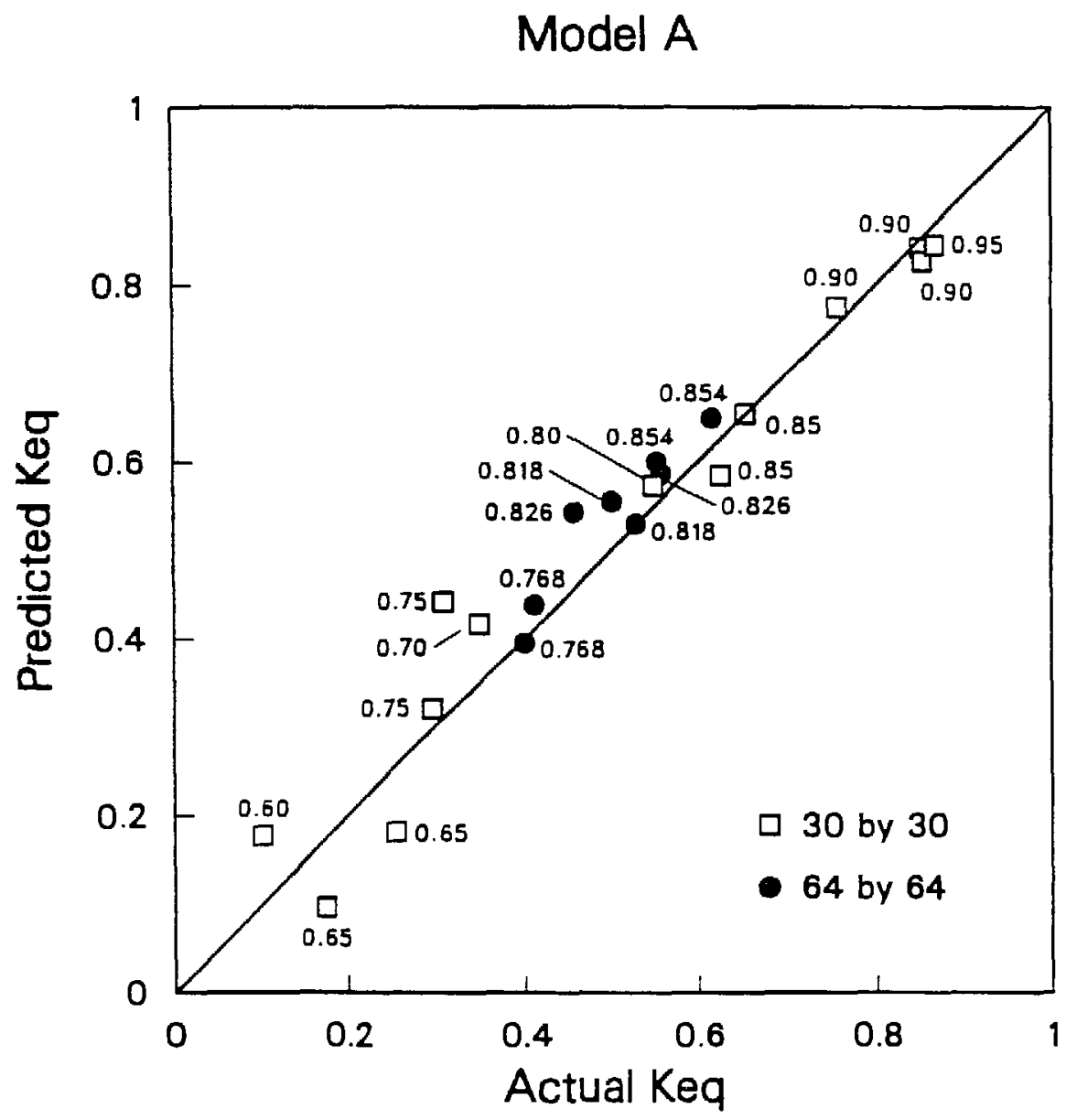

XCG $893-4631$

Figure 6.1.4 Predicted equivalent permeability according to Model A (three statistic extended model) versus actual equivalent permeability for 30 by 30 pixel and 64 by 64 pixel patterns with variable void fractions. Void fractions are indicated on the plot. 
Model B

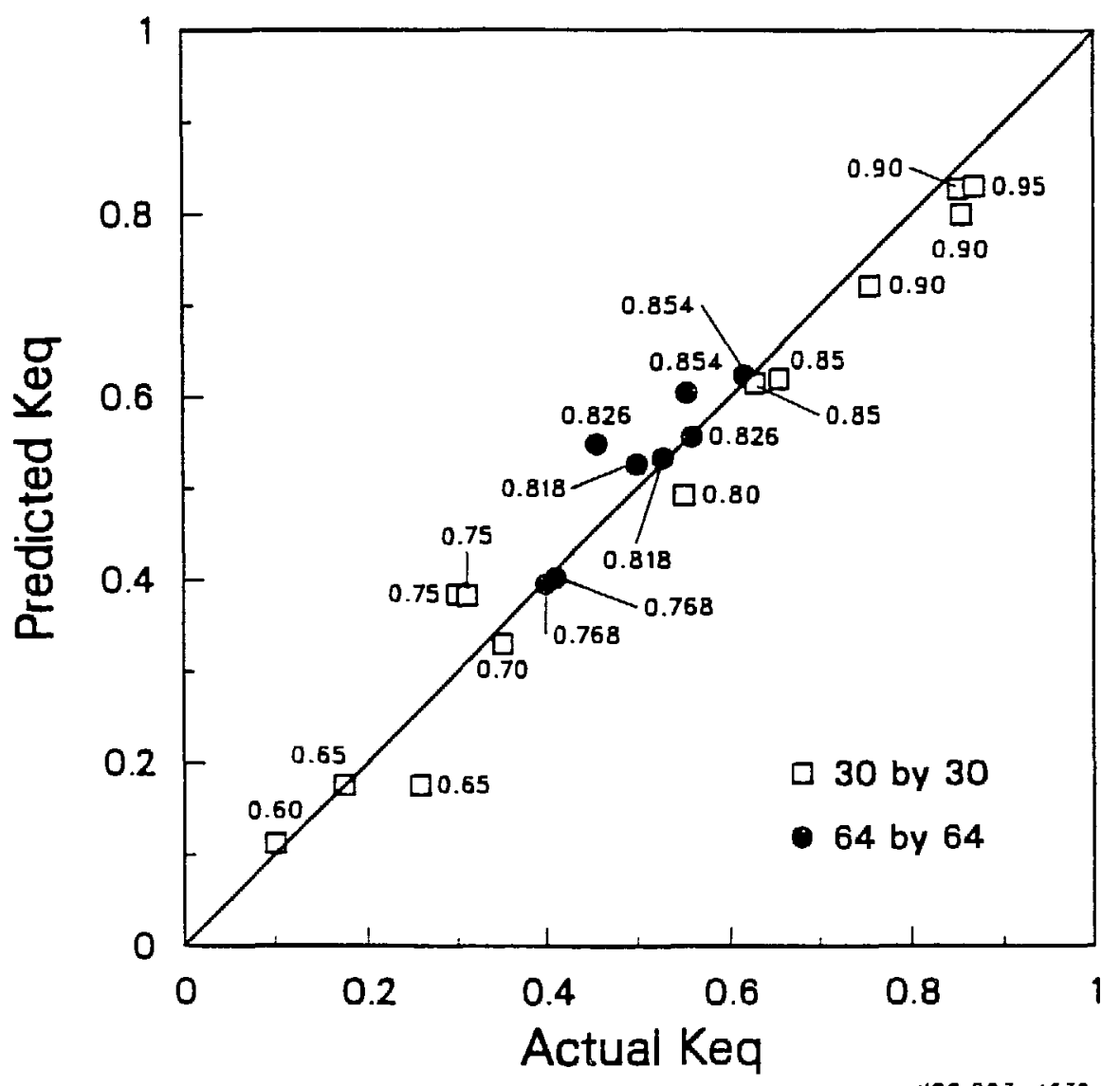

$x C C 893-4632$

Figure 6.1.5 Predicted equivalent permeability according to Model B (NCP extended model) versus actual equivalent permeability for 30 by 30 pixel and 64 by 64 pixel pattems with variable void fractions. Void fractions are indicated on the plot. 


\section{Limitations}

For patterns with very small equivalent permeabilities, Model A and Model B are not appropriate. To demonstrate this, predicted equivalent permeability according to Model A and Model B is plotted for a family of patterns. The patterns in this family result from clipping the same realization of a correlated Gaussian process at different levels. The same realization which was clipped to get the patterns in Figure 6.1.1 is clipped again. Hence, equivalent permeability is known at void fractions $0.60,0.70,0.80,0.90$ and 0.95 . The empirical percolation threshold, i.e. the largest void fraction where equivalent permeability is zero, is 0.478 . Between 0.478 and 0.60 , actual equivalent permeability slowly increases from zero to 0.101 . However, Figure 6.1.6 shows that the Model A and Model B prediction curves dip below the $K_{\text {eq }}=0$ axis to the right of the percolation threshold. Thus, for patterns too near the percolation threshold, both Model A and B are inappropriate. This is not surprising since the estimation error ( $R \hat{S E}$ ) for each extended model is comparable to the equivalent permeability of patterns with void fractions close to the threshold. Since the percolation threshold is known for this family of patterns, it may be possible to predict equivalent permeability using Percolation Theory (Broadbent and Hammersley, 1957) for the range of void fraction where our models are inappropriate. Next, Model $A$ and $B$ are used to predict the equivalent permeability of other patterns.

\subsection{Applications}

Model $\mathrm{A}$ and $\mathrm{B}$ are used to predict the equivalent permeabilities of the 128 by 128 pixel patterns from Figure 3.1 and 3.2 in Chapter 3. Pattern 128a is the observed pattern of voids and contact areas that motivated this study and patterns $128 \mathrm{~b}, 128 \mathrm{c}$, and $128 \mathrm{~d}$ were simulated.

The equivalent permeabilities of the 128 by 128 patterns were determined using the same finite difference method from Chapter 4 . For each pattern, a 256 by 256 


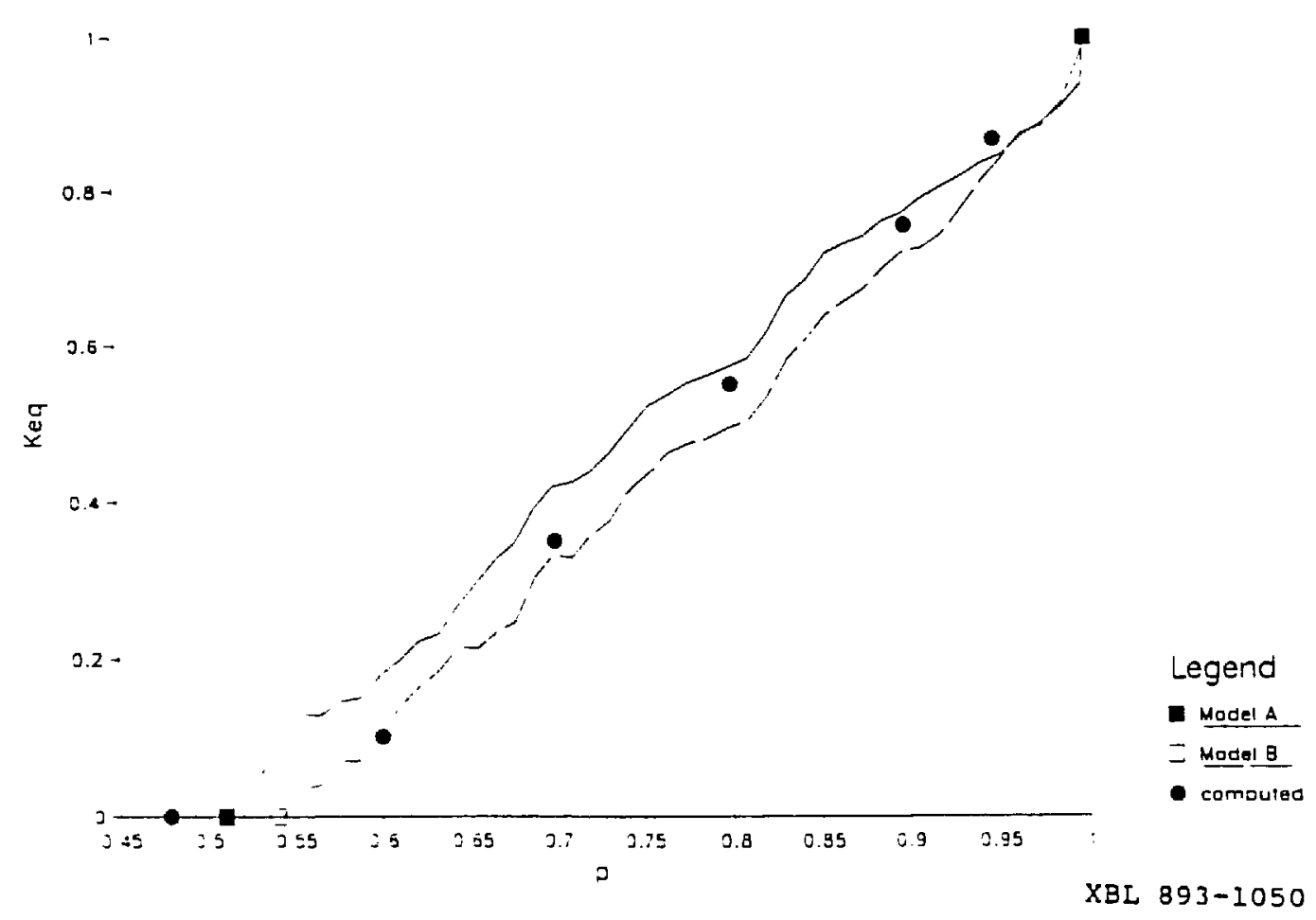

Figure 6.1.6 Model A and Model B predicted equivalent permeability for a family of patterns. The same 30 by 30 pixel realization of a correlated Gaussian defined on a 30 by 30 pixel square region, is clipped at different levels to get different members of the family. Computed equivalent at void fractions of $0.60,0.70,0.80,0.90$ and 0.95 is plotted for comparison. 
mesh was used to determine equivalent permeability. Table 6.2.1 shows how the equivalent permeability of pattern $128 \mathrm{~b}(\hat{y})$ depended on mesh size. Since the variation is just a couple of percent, the 256 by 256 result is precise to within a few percent. Therefore, differences between predicted values and reported values less than a few percent may not be significant.

Table 6.2.1. Equivalent permeability dependence on mesh size for pattern $128 \mathrm{~b}$ (j).

\begin{tabular}{|ccc|}
\hline mesh & iterations & $K_{\text {eq }}$ \\
\hline \hline 128 by 128 & 2000 & 0.548 \\
256 by 256 & 1000 & 0.519 \\
512 by 512 & 200 & 0.548 \\
\hline
\end{tabular}

Table 6.2.2 lists Model A and B predicted equivalent permeability and actual equivalent permeability for each of the 128 by 128 pixel patterns. Figures 6.2 .1 and 6.2.2 show that Model B predicted the equivalent permeability of 128 by 128 patterns better than Model A did.

Table 6.2.2. Predicted and actual equivalent permeabilities for the 128 by 128 pixel patterns.

\begin{tabular}{|lllllll|}
\hline & NCP & NSIGC & NSIGR & $K_{\text {aq }}$ & $\hat{K}_{\text {eq }}{ }^{A}$ & $\hat{K}_{\text {eq }}{ }^{B}$ \\
\hline \hline 128a $(\hat{y})$ & 1.015 & 0.895 & 0.652 & 0.433 & 0.480 & 0.490 \\
128a $(\hat{x})$ & 0.889 & 0.652 & 0.895 & 0.534 & 0.580 & 0.530 \\
128b $(\hat{y})$ & 0.915 & 0.631 & 0.786 & 0.519 & 0.570 & 0.523 \\
128b $(\hat{x})$ & 0.952 & 0.786 & 0.631 & 0.477 & 0.505 & 0.511 \\
128c $(\hat{y})$ & 0.970 & 0.577 & 0.788 & 0.522 & 0.575 & 0.505 \\
128c $(\hat{x})$ & 0.971 & 0.788 & 0.577 & 0.471 & 0.490 & 0.504 \\
128d $(\hat{y})$ & 0.879 & 0.540 & 0.801 & 0.505 & 0.593 & 0.534 \\
128d $(\hat{x})$ & 0.921 & 0.801 & 0.540 & 0.456 & 0.485 & 0.520 \\
\hline
\end{tabular}

Table 6.2.3 lists the root mean square prediction error $(R \dot{S E})$ and average bias $\left.\left(<K_{\text {eq }}-\hat{K}_{\text {eq }}\right\rangle\right)$ for the 30 by 30 pixel, 64 by 64 pixel and 128 by 128 pixel patterns 
Model A

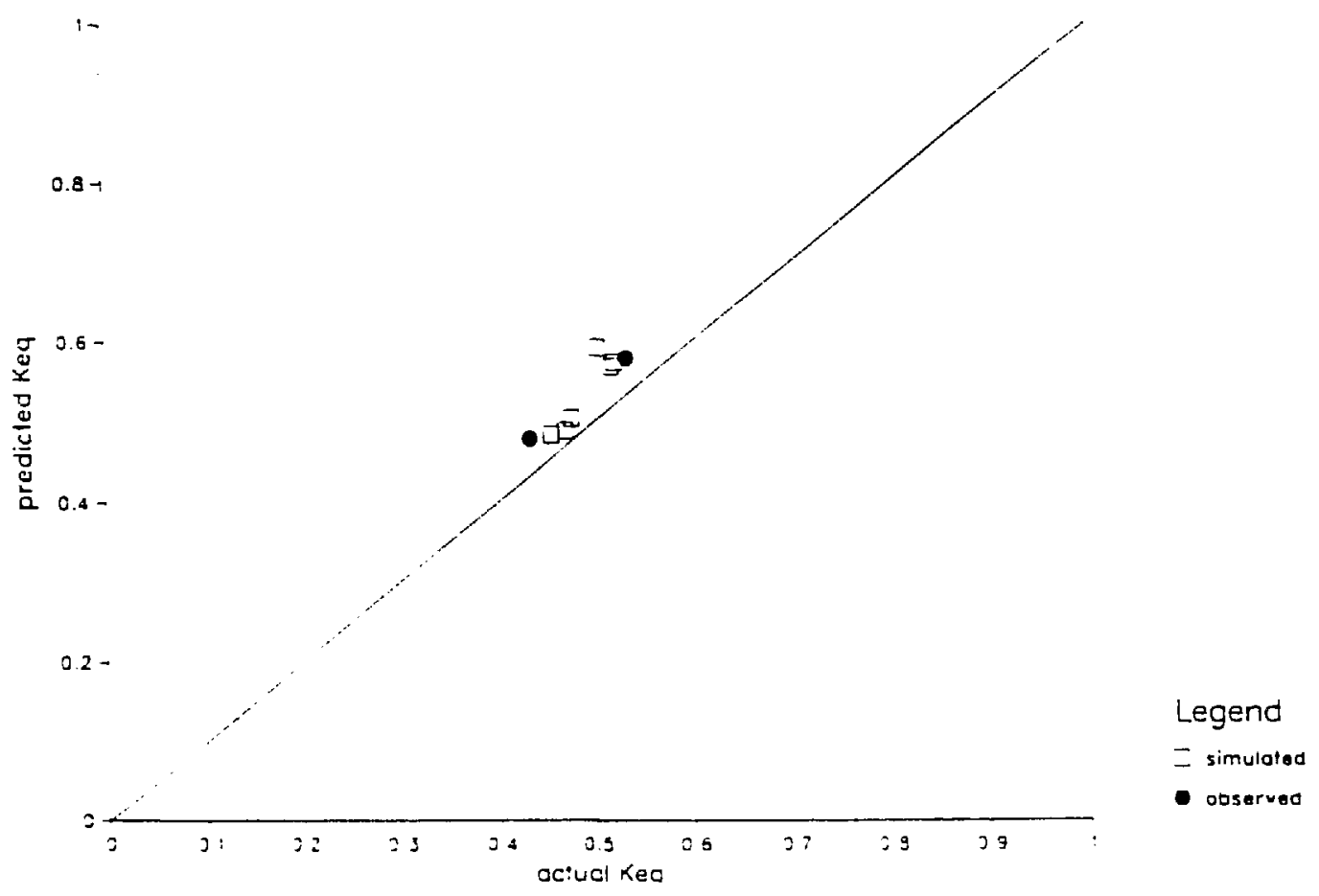

XBL $893-1081$

Figure 6.2.1 Model A prediction for equivalent permeability versus actual equivalent permeability for observed (128a) and simulated (128b, 128c, 128d) 128 by 128 pixel patterns. 
Model B

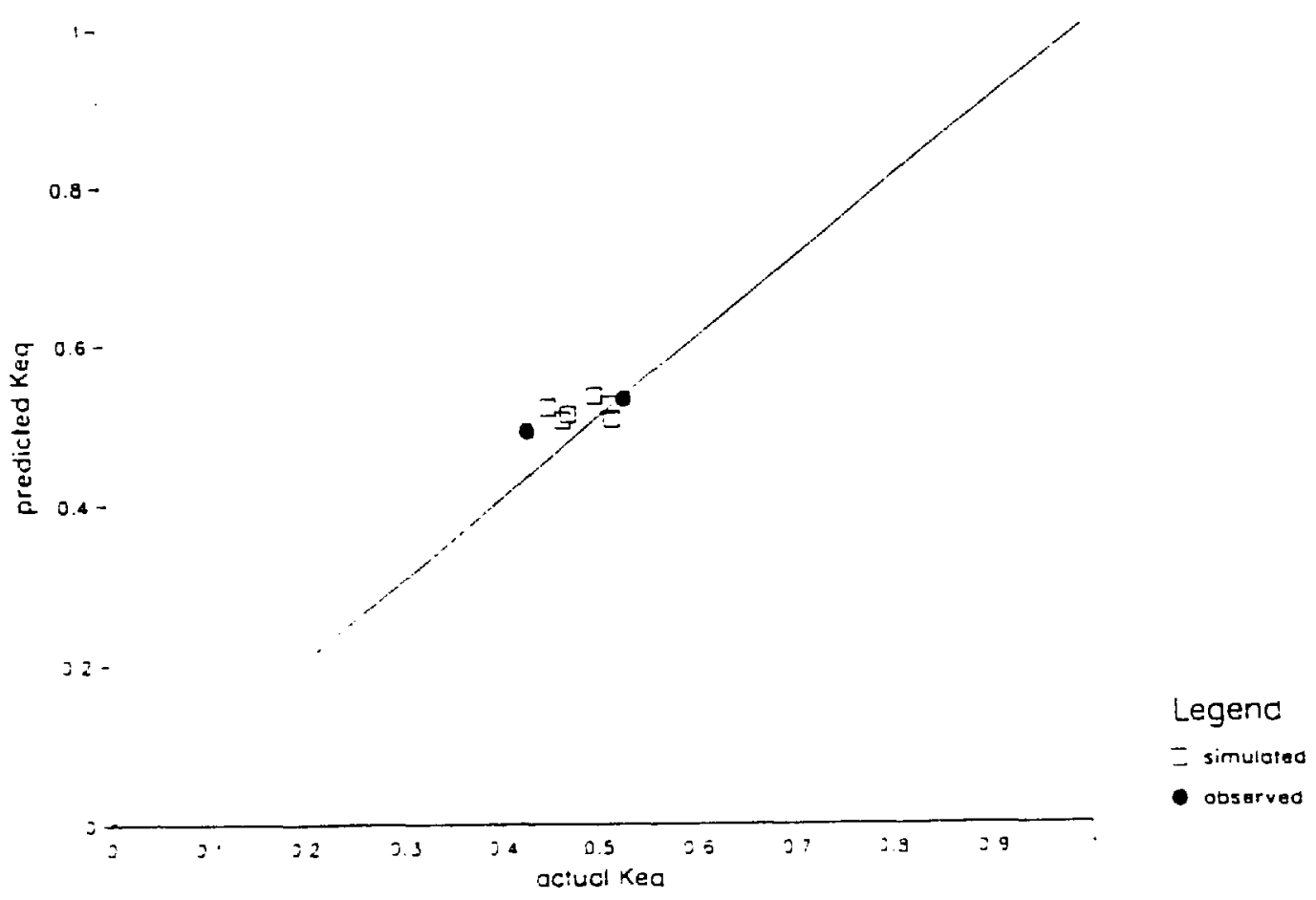

XBL 893-1093

Figure 6.2.2 Model B prediction for equivalent permeability versus actual equivalent permeability for observed (128a) and simulated (128b, 128c, 128d) 128 by 128 pixel pattems. 
considered in this section. For each group of patterns, Model $\mathrm{B}$ had a lower $R \hat{S} E$. Note that even though the $R \hat{S E}$ for Model B decreases as the scale of the simulated patterns increases, bias for Model B increases as scale increases. As scale increases, the simulated patterns become more homogeneous. Hence, if the model parameters are estimated precisely and do not depend on scale, both $R \hat{S E}$ and bias should decrease. The slight increase of bias of Model B with scale indicates that the fitted model parameters may depend on scale.

Table 6.2.3 Comparison of root mean square prediction error and bias of Model $A$ and Model $B$ for 30 by 30,64 by 64 and 128 by 128 pixel patterns from Chapter 3 model.

\begin{tabular}{|lllll|}
\hline & RSE $_{\text {A }}$ & RSE $_{B}$ & $\langle\text { Bias }\rangle_{A}$ & $\langle\text { Bias }\rangle_{B}$ \\
\hline \hline 30 by 30 & 0.058 & 0.048 & 0.006 & -0.014 \\
64 by 64 & 0.044 & 0.039 & 0.035 & 0.024 \\
128 by 128 & 0.050 & 0.037 & 0.046 & 0.025 \\
\hline
\end{tabular}

For even larger simulated patterns from the Chapter 3 model, there is reason to expect that Model B will predict equivalent permeability well. This is because NCP is statistical replication invariant and because Desbarats (1987) showed in a numerical study that the effective permeability of a pattern of high and low permeability regions with exponential correlogram stabilized as pattern size increased. However, this does not mean that the model will work for all large patterns. How well the model works for more complex patterns, or patterns simulated differently, is an open question. Partial answers to this open question are provided next.

\section{Different Moving Average Filter}

Instead of simulating patterns with a moving average with $\kappa=0.75$ as before, $k$ was changed. With $\kappa=0.25$, four different 30 by 30 pixel realizations of a correlated Gaussian process were clipped. These new patterns 30v, 30w, 30x, 30y are shown in 
Figure 6.2.3. With $\kappa=1.25$, four different 30 by 30 pixel realizations of a correlated Gaussian process were clipped. These new patterns 30z, 30aa, 30bb, 30cc are shown in Figure 6.2.4.

Tables 6.2.4 and 6.2.5 list the statistics of the patterns and the predicted and actual equivalent permeabilities according to Model A and Model B. Equivalent permeability was determined using the same technique as was used for the other 30 by 30 patterns in this section; a 150 by 150 finite difference mesh was used.

Table 6.2.4. Predicted and actual equivalent permeability for 30 by 30 simulated patterns simulated with moving average filter parameter $\mathrm{x}=0.25$.

\begin{tabular}{|c|c|c|c|c|c|c|c|}
\hline & $p$ & $N C P$ & NSIGC & NSIGR & $K_{e q}$ & $\overline{\hat{K}_{e q}{ }^{A}}$ & $\hat{K}_{e q}^{B}$ \\
\hline $30 v(\hat{y})$ & 0.650 & 1.017 & 0.566 & 0.496 & 0.252 & 0.152 & 0.270 \\
\hline $30 w(\hat{y})$ & 0.750 & 0.735 & 0.538 & 0.668 & 0.586 & 0.451 & 0.498 \\
\hline $30 \times(\hat{y})$ & 0.850 & 1.131 & 0.370 & 0.719 & 0.734 & 0.652 & 0.497 \\
\hline 30y (̂े) & 0.950 & 0.667 & 0.458 & 0.372 & 0.860 & 0.819 & 0.763 \\
\hline
\end{tabular}

Table 6.2.5. Predicted and actual equivalent permeability for 30 by 30 simulated patterns simulated with moving average filter parameter $\mathrm{k}=1.25$.

\begin{tabular}{|lccccccc|}
\hline & $p$ & NCP & NSIGC & NSIGR & $\boldsymbol{K}_{\text {eq }}$ & $\hat{K}_{\text {eq }}{ }^{A}$ & $\hat{K}_{\text {eq }}{ }^{B}$ \\
\hline \hline 30z $(\hat{y})$ & 0.650 & 1.269 & 0.447 & 0.576 & 0.127 & 0.050 & 0.150 \\
30aa $(\hat{y})$ & 0.750 & 1.073 & 0.587 & 0.827 & 0.387 & 0.434 & 0.398 \\
30bb $(\hat{y})$ & 0.850 & 0.753 & 0.678 & 0.480 & 0.529 & 0.636 & 0.597 \\
30cc $(\hat{y})$ & 0.950 & 0.533 & 0.487 & 0.431 & 0.870 & 0.834 & 0.829 \\
\hline
\end{tabular}

Figures 6.2.5 and 6.2.6 plot predicted versus actual equivalent permeability for these patterns simulated with $\kappa$ equal to 0.25 and 1.25 for both Model $A$ and Model B. Table 6.2.6 lists the root mean square prediction error $(R \hat{S E})$ and average bias for the 30 by 30 pixel patterns simulated with $\kappa=0.25, \kappa=0.75$ and $\kappa=1.25$. 

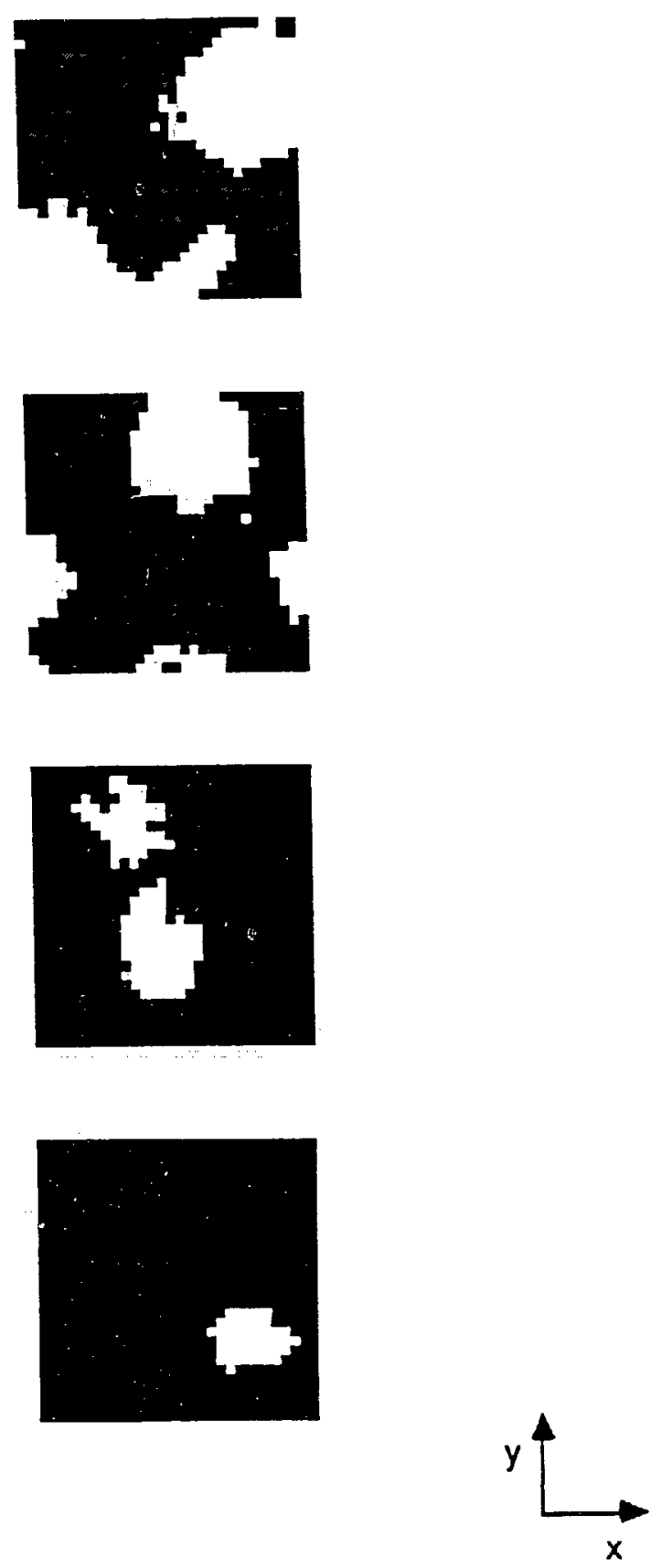

XBL 893-1092

Figure 6.2.3 From the top, simulated 30 by 30 pixel patterns $30 v, 30 w, 30 x, 30 y$. Void fractions are $0.65,0.75,0.85$ and 0.95 . Voids have constant aperture. Moving average filter parameter $(x)$ is 0.25 . 

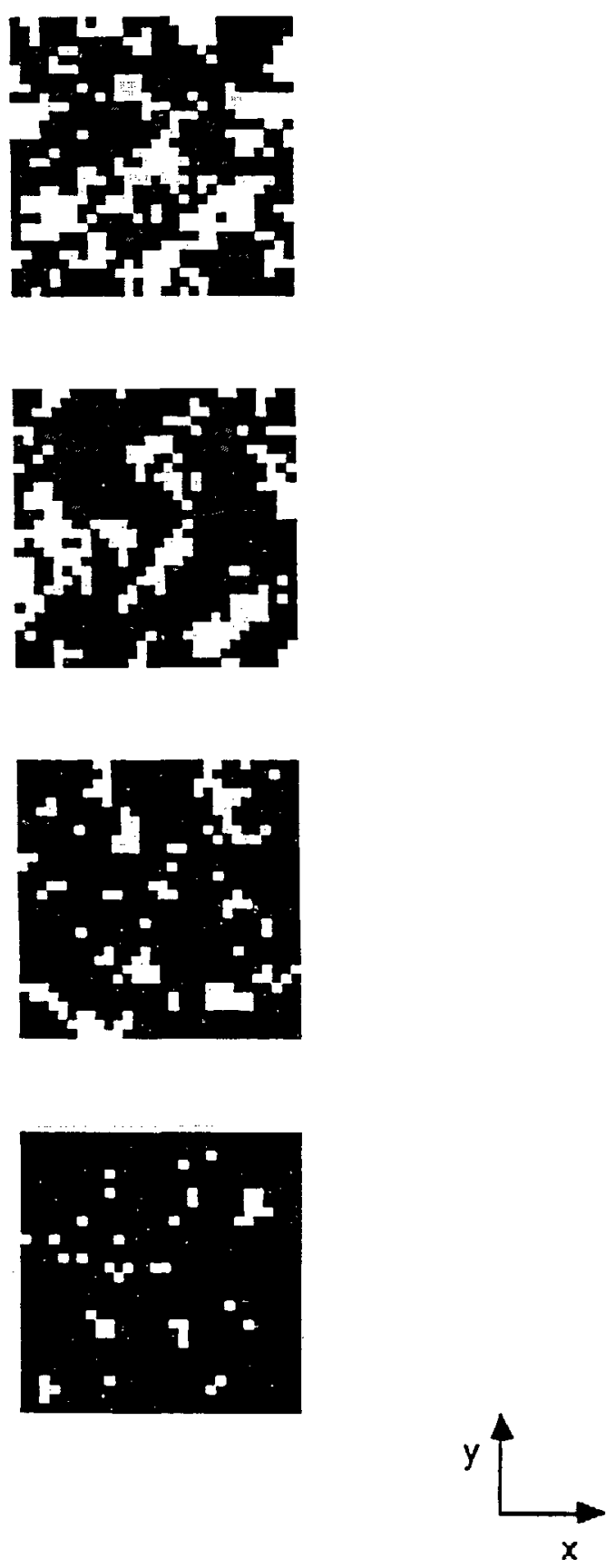

XBL 893-1091

Figure 6.2 .4 From the top, simulated 30 by 30 pixel patterns $30 \mathrm{z}, 30 \mathrm{aa}, 30 \mathrm{bb}, 30 \mathrm{cc}$. Void fractions are $0.65,0.75,0.85$ and 0.95 . Voids have constant aperture. Moving average filter parameter $(\kappa)$ is 1.25 . 


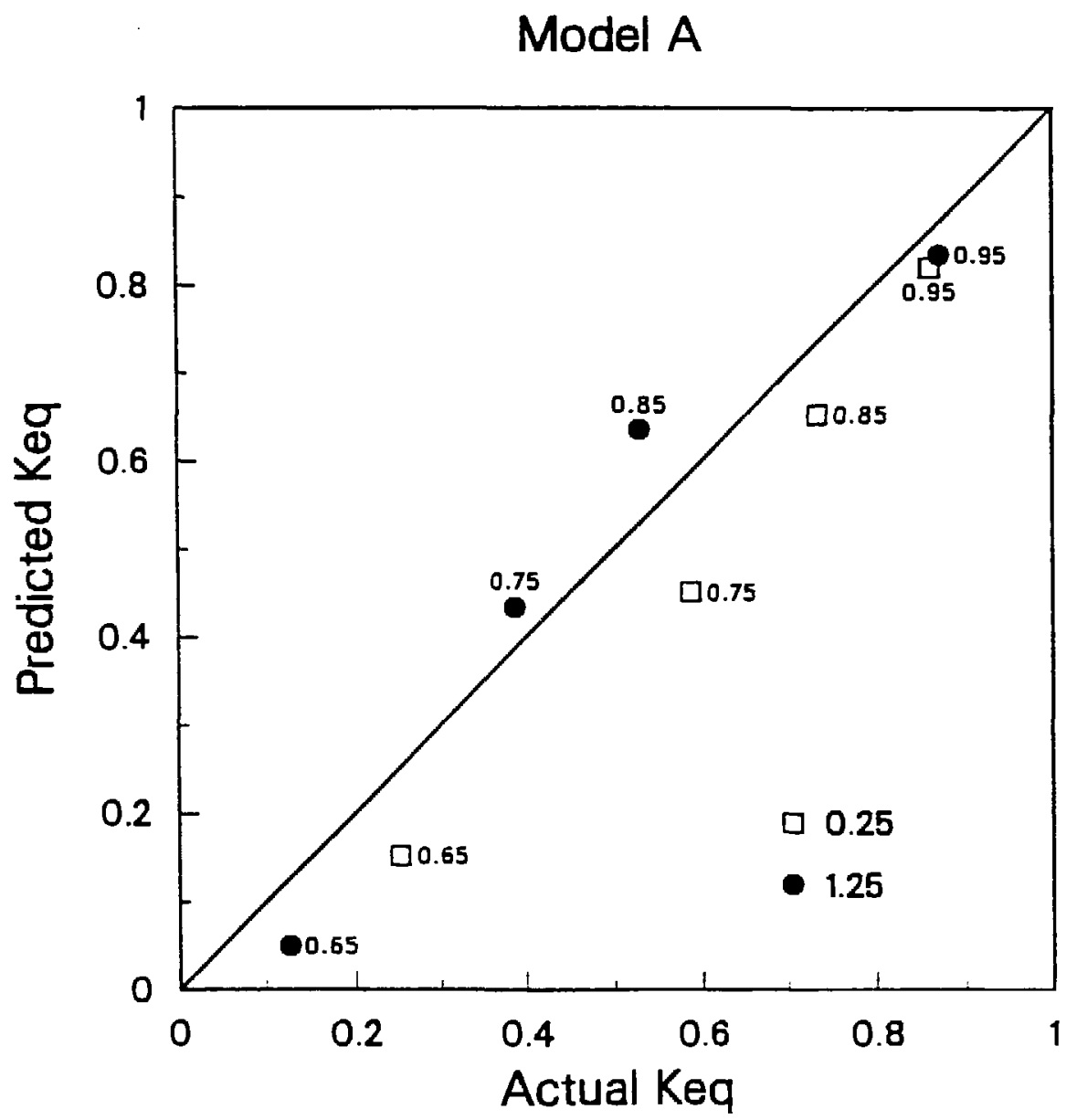

Figure 6.2.5 Model A predicted equivalent permeability versus actual equivalent permeability for simulated 30 by 30 pixel patterns shown in Figures 6.2 .3 and 6.2.4. For each pattern, the moving average filter paramerer $(\kappa)$ was 0.25 or 1.25 . 
Model B

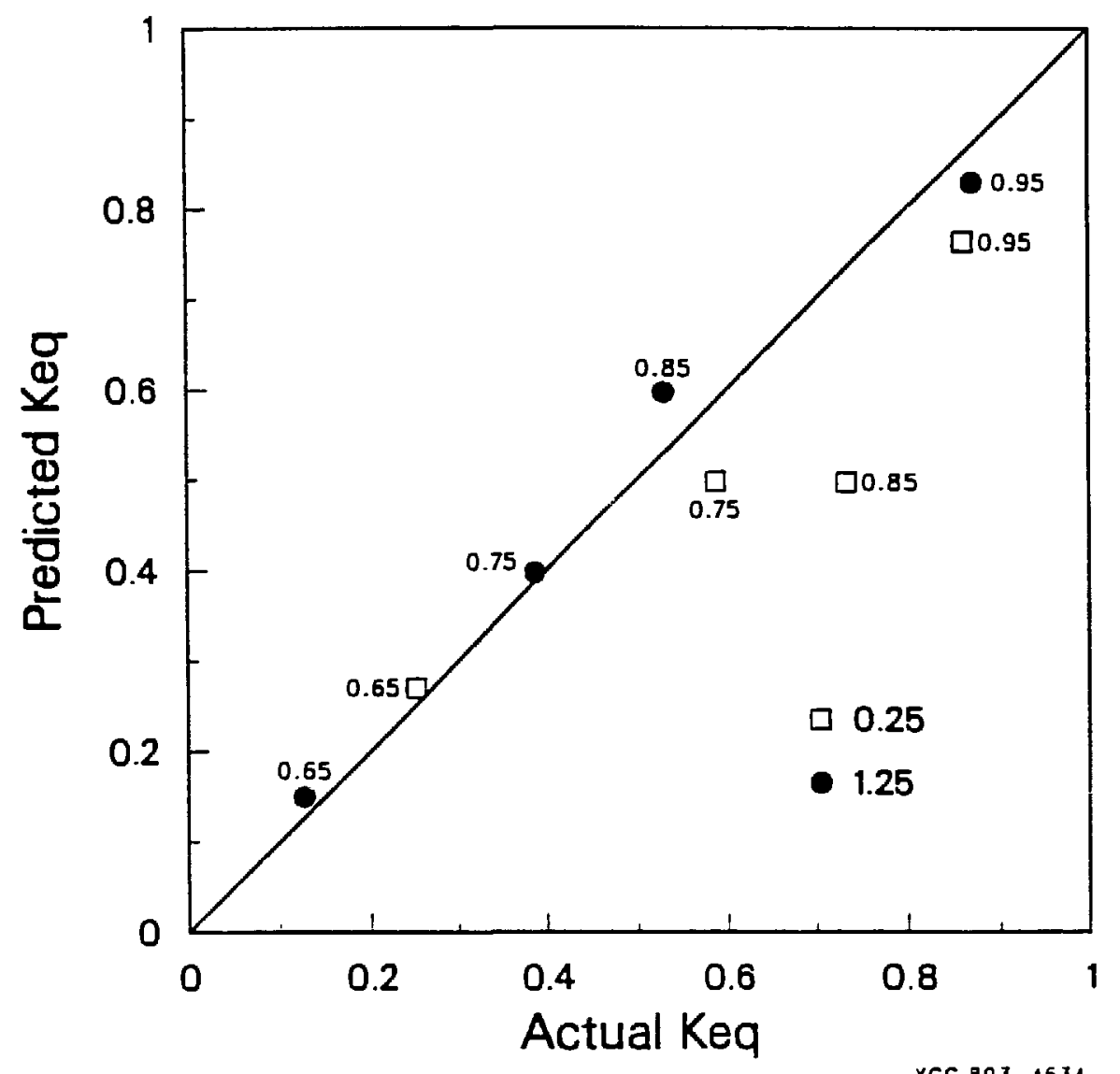

Figure 6.2.6 Model B predicted equivalent permeability versus actual equivalent permeability for simulated 30 by 30 pixel patterns shown in Figures 6.2.3 and 6.2.4. For each pattern, the moving average filter parameter ( $\kappa$ ) was 0.25 or 1.25 . 
Table 6.2.6. Comparison of $R \hat{S} E$ and bias of Model $A$ and Model $B$ for 30 by 30 pixel patterns simulated using different moving average filters.

\begin{tabular}{|ccccc|}
\hline$\kappa$ & $R \hat{S} E_{A}$ & $R \hat{S} E_{B}$ & $\langle\text { Bias }\rangle_{A}$ & $\langle\text { Bias }\rangle_{B}$ \\
\hline \hline 0.25 & 0.096 & 0.136 & 0.089 & 0.101 \\
0.75 & 0.058 & 0.048 & -0.006 & 0.014 \\
1.25 & 0.072 & 0.042 & -0.010 & -0.015 \\
\hline
\end{tabular}

In summary, except for the $\kappa=0.25$ patterns, Table 6.2 .6 shows that Model B predicted equivalent permeability better than Model A did. Further, for the $\mathrm{k}=0.25$ patterns, Model B did well except for pattem 30x $(\hat{y})$. Roughly speaking, Model A did equally well for all the $k=0.25$ patterns. Perhaps the $k=0.25$ patterns were not as consistently well predicted because of a scale effect. That is, it may be harder in general, to predict the flow through simulated patterns with just a few random fearures than for more homogeneous simulated patterns with many features. As $\mathrm{k}$ increased, and patterns became more homogeneous, the $R \hat{S} E$ and bias for Model B decreased. This is consistent with the notion that homogeneous pattems are easier to predict and that the fitted parameters for Model B are not sensitive to $x$. However, for Model A, $R \hat{S E}$ does not monotonically decrease as $\mathrm{K}$ increases; $R \hat{S E}$ is lowest for the $\kappa=0.75$ pattems. Note that the Model A parameters were found by fitting the model to data from simulated patterns obtained from the Chapter 3 model with $k=0.75$. Hence, the fitted parameters for Model A may depend on $\mathrm{K}$. In summary, based on evidence so far, Model B appears to be the more generally applicable extended model. As a further check, the next section compares how well the Models predict the equivalent permeability of patterns simulated using fractal ideas.

\section{Fractals}

Fractal patterns (Mandelbrot, 1983) are self similar. For instance, if a fractal pattem were photographed, the picture would look the same regardless of the magnification scale. Thus, a fractal rock fracture would have contact area features at 
all scales. Below, Figure 6.2.7 shows an example of a fractal. The last pattern in the infinite sequence of patterns that begins with the three shown here is the Sierpinski Carpet. The figure is from Stauffer (1985).

Random patterns of voids and contact areas are simulated in two ways. Following an idea by Nolte et al. (1987), we simulate random Sierpinski carpets. In the first simulation method, randomly located rectangular void region are cut out, in successive stages, of a 64 by 64 pixel pattern with an initial void fraction of unity. In the first stage, a randomly located 32 by 16 pixel rectangle of voids is cut out. In the second stage, a randomly located 8 by 4 pixel rectangle is cut out each of the $4^{2} 16$ by 16 pixel subquadrants in the pattern. In the third and final stage, a randomly located 2 by 1 pixel rectangle is cut out of each of the $16^{2} 4$ by 4 pixel sub-subquadrants in the pattern. Rectangles can overlap. Pattern 64fa, shown in Figure 6.2.8, was simulated by the above method. Figure 6.2.8 also shows pattern 30fa which is the upper left hand 30 by 30 pixel corner of pattern $64 \mathrm{fa}$. None of the patterns simulated in this work are true fractals because the contact area features have a limited size range. Hence, the simulated patterns are quasi-fractal.

For each of these patterns, equivalent permeability is computed for both pressure drop directions using the same methods as used for the other 30 by 30 and 64 by 64 pixel patterns in this Chapter. Table 6.2.7 shows that Model B does a better job of predicting flow than does Model B. In particular, for flow in the $x$ direction across pattern 64a, Model A is way off. 


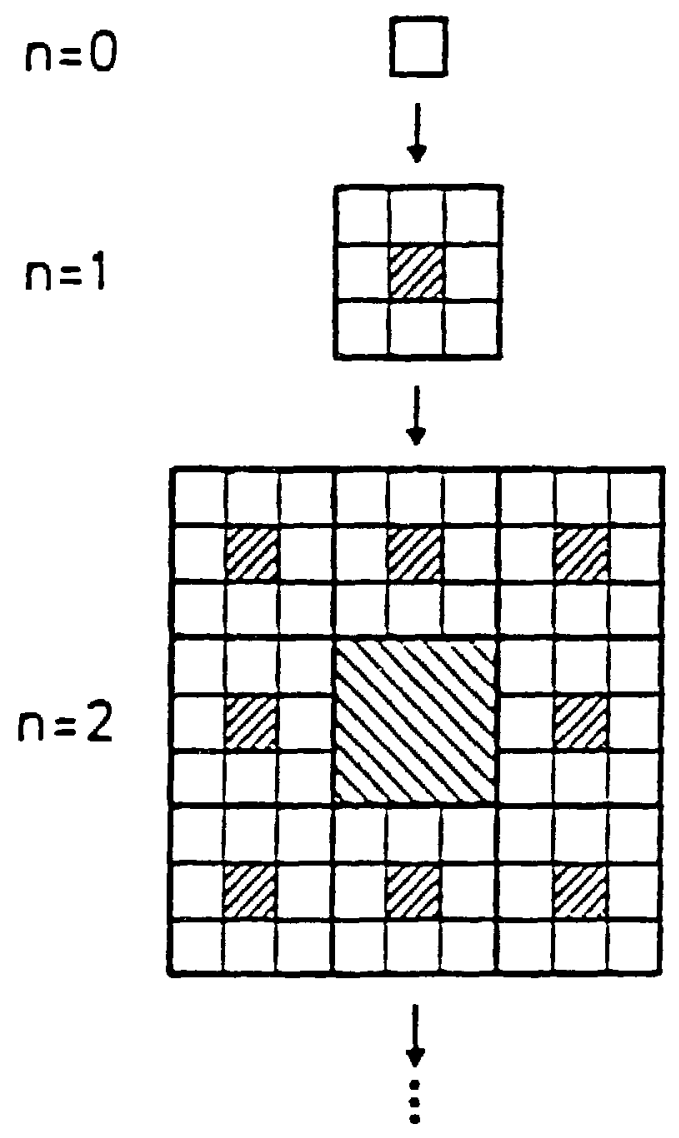

XBL $893-1090$

Figure 6.2.7 The buildup of the Sierpinski carpet fractal. The $n=\infty$ member of the above sequence is a fractal. Figure is from Stauffer (1985). 

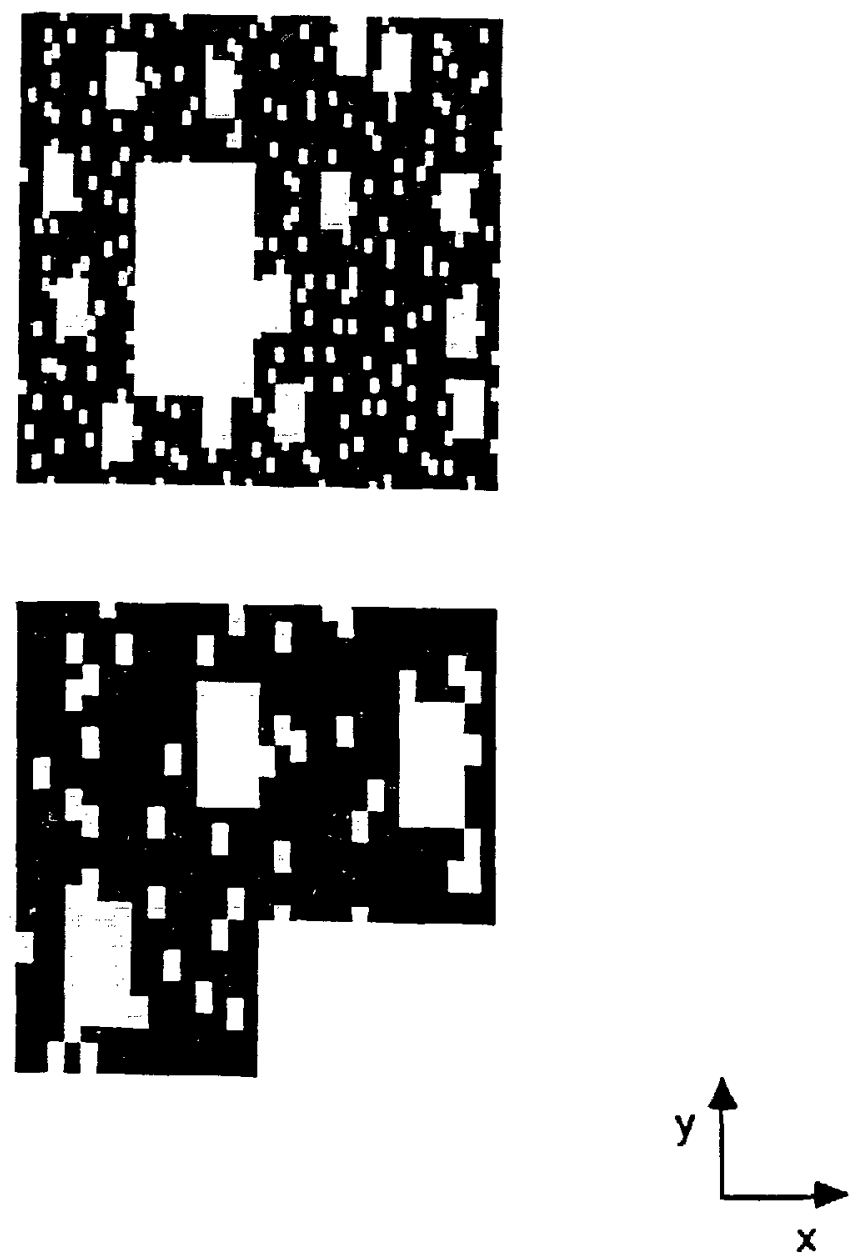

XBL 893-1089

Figure 6.2.8 Patterns $64 \mathrm{fa}$ and $30 \mathrm{fa}$ are simulated using fractal ideas. Black pixels are voids and white pixels are contact. Void fractions are 0.667 and 0.631 . 
Table 6.2.7. Predicted and computed equivalent permeability for patterns 64fa and $30 \mathrm{fa}$.

\begin{tabular}{|lccccccc|}
\hline & $P$ & $N C P$ & NSIGC & NSIGR & $K_{\text {eq }}$ & $\hat{K}_{\text {eq }}{ }^{A}$ & $\hat{K}_{\text {eq }}{ }^{B}$ \\
\hline \hline 64fa $(\hat{y})$ & 0.667 & 0.608 & 0.999 & 1.466 & 0.485 & 0.412 & 0.444 \\
64fa $(\hat{x})$ & 0.667 & 1.162 & 1.466 & 0.999 & 0.258 & 0.041 & 0.240 \\
30fa $(\hat{y})$ & 0.631 & 0.635 & 1.115 & 0.985 & 0.384 & 0.144 & 0.391 \\
30fa $(\hat{x})$ & 0.631 & 1.143 & 0.985 & 1.115 & 0.296 & 0.159 & 0.198 \\
\hline
\end{tabular}

To determine if the performance of Model A improves for patterns with larger void fractions, pattem 64fb, shown in Figure 6.2 .9 below, was simulated in a manner almost identical to the first pattern except that the rectangles were half as wide as before. This pattern had void fraction equal to 0.819 . The upper left hand 30 by 30 pixel corner of pattern 64fb is displayed in Figure 6.2.9 as pattem 30fb.

As conjectured, Table 6.2.8 shows that Model A predicts equivalent permeability better for these patterns. Model A should do better for patterns with void fractions near 0.80 because it agrees with Eq. 6.1.1 when void fraction equals 0.80 .

Table 6.2.8. Predicted and computed equivalent permeability for patterns $64 \mathrm{fb}$ and $30 \mathrm{fb}$.

\begin{tabular}{|cccccccc|}
\hline & $p$ & $N C P$ & NSIGC & NSIGR & $K_{\text {aq }}$ & $\hat{K}_{\text {aq }}{ }^{A}$ & $\hat{K}_{\text {eq }}{ }^{B}$ \\
\hline \hline 64fb $(\hat{y})$ & 0.819 & 0.545 & 0.804 & 1.591 & 0.698 & 0.729 & 0.644 \\
64fb $(\hat{x})$ & 0.819 & 0.770 & 1.591 & 0.804 & 0.452 & 0.398 & 0.572 \\
30fb $(\hat{y})$ & 0.788 & 0.558 & 0.936 & 1.082 & 0.636 & 0.543 & 0.603 \\
30fb $(\hat{x})$ & 0.788 & 0.818 & 1.082 & 0.936 & 0.485 & 0.453 & 0.517 \\
\hline
\end{tabular}

For completeness, the next section investigates how well the models do for quasifractal patterns simulated in a different way.

\section{Another kind of fructal}

In the first simulation technique, contact area regions were cut out of a sheet of voids. In the second method, conducting void regions are thrown down on a originally non-conducting sheet. Like in the first simulation, the second kind of patterns 

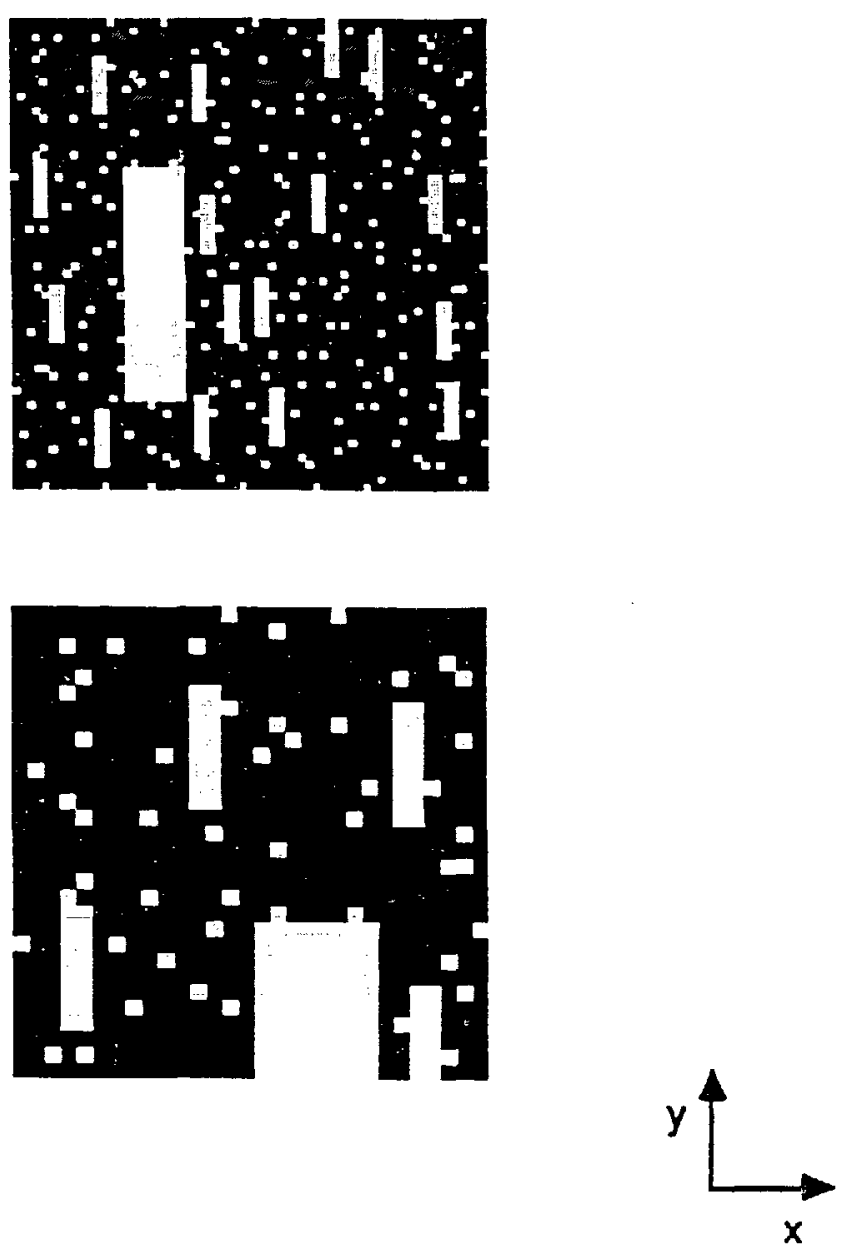

XBL 893-1088

Figure 6.2.9 Patterns $64 \mathrm{fb}$ and $30 \mathrm{fb}$ are simulated using fractal ideas. Black pixels are voids and white pixels are contact. Void fractions are 0.819 and 0.788 . 
are simulated in three stages. In the first stage, five randomly located 32 by 16 void regions are thrown down. In the second stage, five randomly located 8 by 4 pixel void regions are thrown down on each of the $4^{2} 16$ by 16 pixel subquadrants of the pattern. In the last stage, five randomly located 2 by 1 void regions are thrown down onto each of the $16^{2} 4$ by 4 sub-subquadrants in the pattern. Figure 6.2 .10 shows two 64 by 64 pixel patterns simulated this way-- patterns $64 \mathrm{fc}$ and $64 \mathrm{fd}$.

Table 6.2.9 shows that Model A and Model B predicted equivalent permeability reasonablely well except for flow in the $x$ direction across pattern $64 d$.

Table 6.2.9 Predicted and computed equivalent permeability for patterns $64 \mathrm{fc}$ and $64 \mathrm{fd}$.

\begin{tabular}{|lccccccc|}
\hline & $p$ & $N C P$ & $N S I G C$ & NSIGR & $K_{\text {eq }}$ & $\hat{K}_{\text {eq }}{ }^{A}$ & $\hat{K}_{\text {eq }}{ }^{B}$ \\
\hline \hline 64fc $(\hat{y})$ & 0.865 & 0.605 & 0.837 & 0.987 & 0.630 & 0.685 & 0.680 \\
64fc $(\hat{x})$ & 0.865 & 1.084 & 0.987 & 0.837 & 0.464 & 0.603 & 0.533 \\
64fd $(\hat{y})$ & 0.837 & 0.709 & 0.945 & 1.080 & 0.566 & 0.621 & 0.613 \\
64fd $(\hat{x})$ & 0.837 & 1.212 & 1.080 & 0.945 & 0.255 & 0.531 & 0.455 \\
\hline
\end{tabular}

Note that in pattern $64 \mathrm{fd}$, flow is almost blocked in the $x$ direction. We conjecture that the disagreement between predicted and actual flow is due to the spatial statistics being computed from all the voids and contact areas in the pattern, whereas flow is passing only through a limited part of the pattern. Consequently, if more channels for flow existed in the $x$ direction, the agreement should be better.

To check out this conjecture, pattem $64 \mathrm{fd}$ was altered. In the first altered pattern 64fe, shown in Figure 6.2.11, all contact area pixels in rows 8 and 56 were converted to voids. In the second altered pattern 64ff, also shown in Figure 6.2.11, contact area pixels in rows $8,16,24,32,40,48$, and 56 were converted to voids. The alterations changed void fraction by a minute amount but dramatically increased flow. Furthermore, agreement between the predicted and actual equivalent permeability improved as more flow channels were created. 

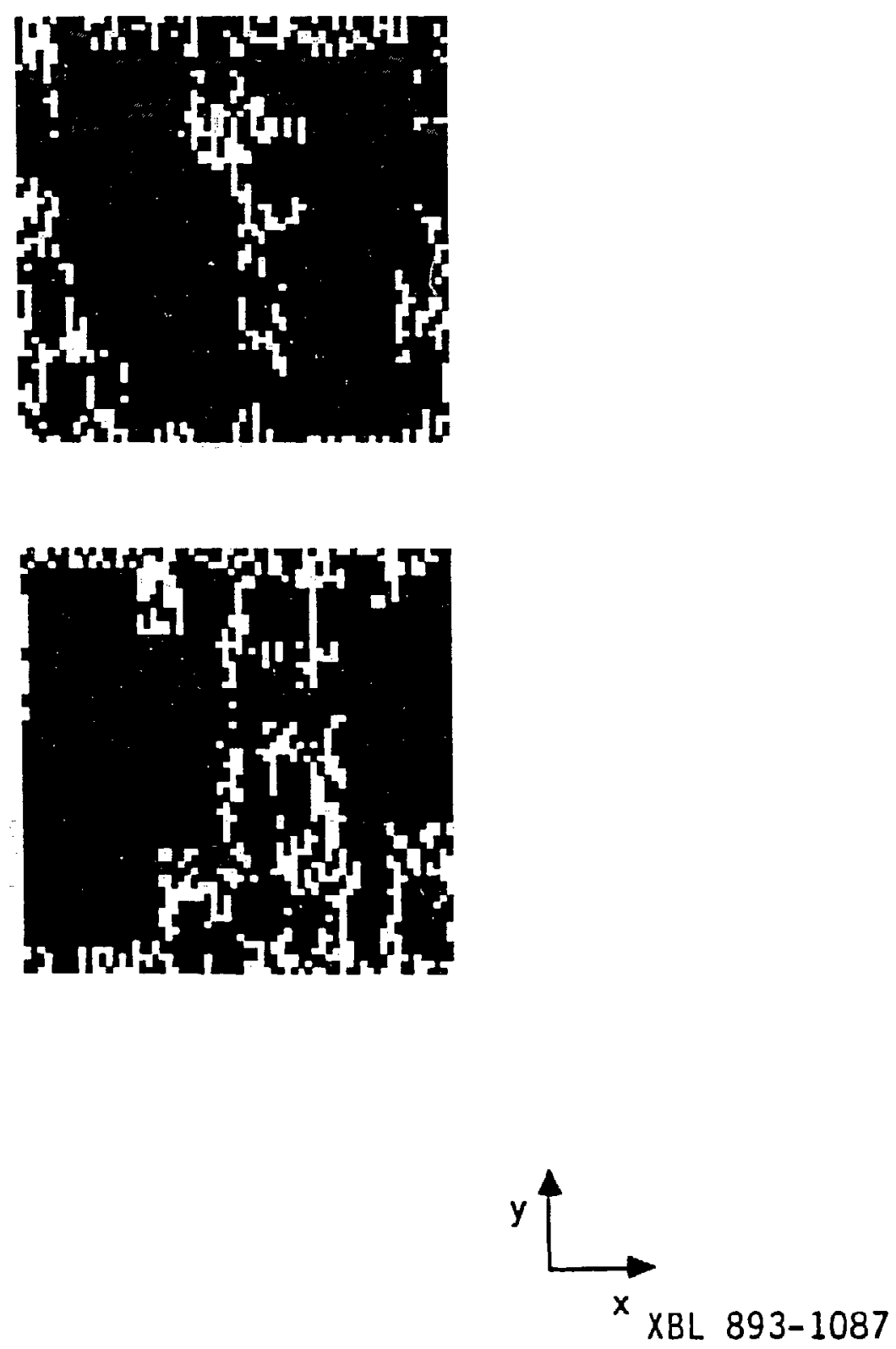

Figure 6.2.10 Pattems $64 \mathrm{fc}$ and $64 \mathrm{fd}$ are simulated using fractal ideas. Black pixels are voids and white pixels are contact. Void fractions are 0.865 and 0.837 . 

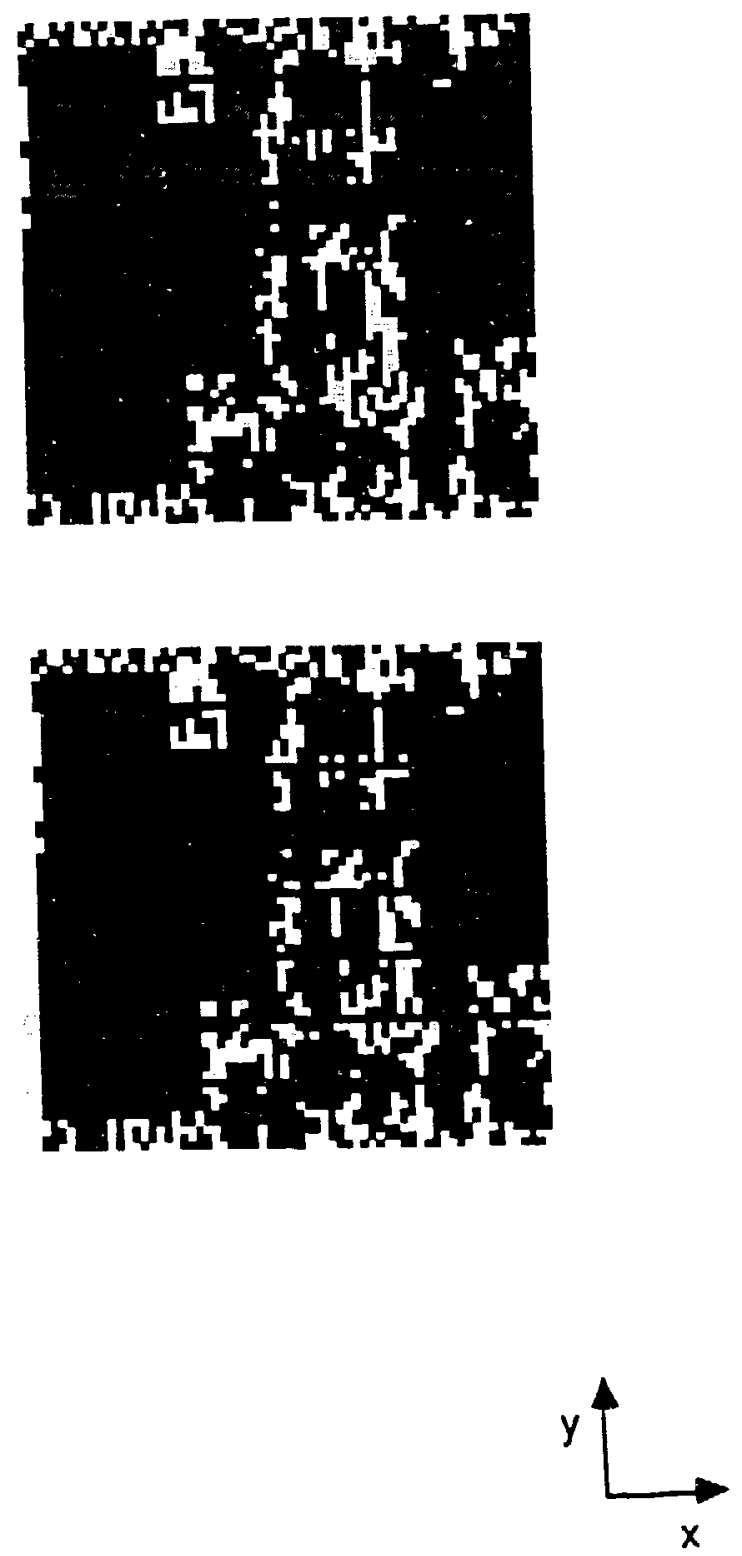

XBL 893-1086

Figure 6.2.11 Pattems 64fe and 64ff are modified versions of $64 \mathrm{fd}$. Void fractions are 0.842 and 0.852 . 
Table 6.2.10 Predicted and computed equivalent permeability for patterns $64 \mathrm{fd}$, 64fe and 64ff.

\begin{tabular}{|lccccccc|}
\hline & $p$ & $N C P$ & $N S I G C$ & $N S I G R$ & $K_{\text {eq }}$ & $\hat{K}_{\text {eq }}{ }^{A}$ & $\hat{K}_{e q}{ }^{B}$ \\
\hline \hline 64fd $(\hat{x})$ & 0.837 & 1.212 & 1.080 & 0.945 & 0.255 & 0.531 & 0.455 \\
64fe $(\hat{x})$ & 0.842 & 1.141 & 1.072 & 1.002 & 0.393 & 0.560 & 0.484 \\
64ff $(\hat{x})$ & 0.852 & 1.008 & 1.040 & 1.109 & 0.516 & 0.615 & 0.538 \\
\hline
\end{tabular}

Another example of this kind of disagreement is easy to imagine. Suppose that a 1000 by 1000 pixel pattern had its equivalent permeability well predicted by our model. Now, if all the voids in one column in the direction perpendicular to the applied pressure drop are converted into contact area pixels, equivalent permeability drops to zero. However, the spatial statistics would only be slightly perturbed because at most only $0.1 \%$ of the voids were changed into contact areas. Thus, one column can dramatically affect fluid flow through the whole pattern while only slightly perturbing the spatial statistics.

\section{Fractal Summary}

For the patterns simulated using fractal ideas, Figure 6.2.12 plots predicted versus actual equivalent permeability for Model A. Figure 6.2.13 shows the same for Model $\mathbf{B}$. Comparison of mean square prediction errors confirms the visual evidence; $R \hat{S E}$ for Model A was 0.143 and 0.079 for Model B. For all the fractal patterns, Table $\tilde{G} .2 .11$ lists equivalent permeability and prediction error $\left(\varepsilon=K_{e q}-\hat{K}_{\varepsilon q}\right)$ for the two modelis. 
Model A

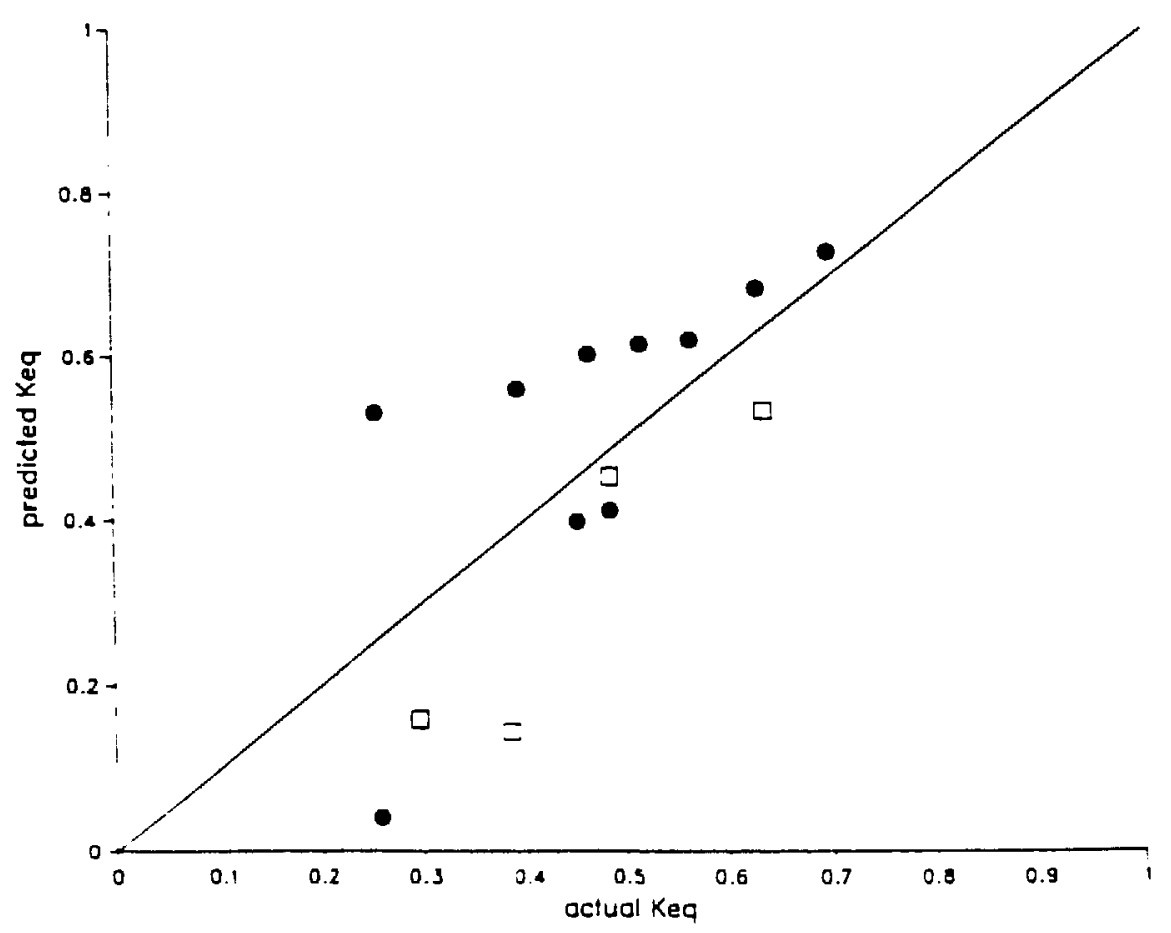

Legend

= 30 by 30

- 64 by 64

XBL $893-1085$

Figure 6.2.12 Model A predicted equivalent permeability versus actual equivalent permeability for 30 by 30 and 64 by 64 pixel patterns simulated using fractal ideds. 
Model B

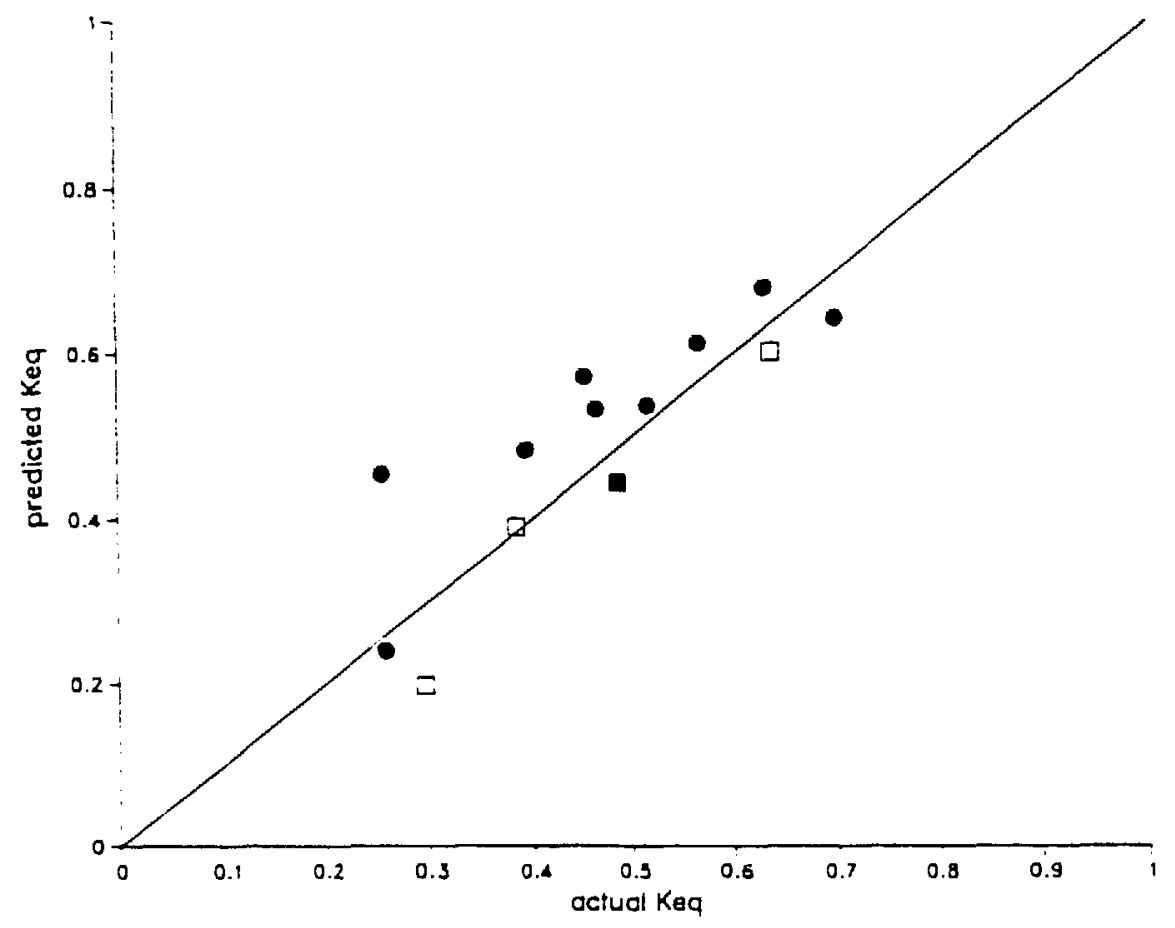

Legend

30 by 30

64 by 64

XBL 893-1084

Figure 6.2.13 Model B predicted equivalent permeability versus actual equivalent permeability for 30 by 30 and 64 by 64 pixel patterns simulated using fractal ideas. 
Table 6.2.11. Prediction errors for 30 by 30 and 64 by 64 pixel patterns simulated using fractal ideas.

\begin{tabular}{|ccccc|}
\hline & $p$ & $K_{\text {aq }}$ & $\varepsilon^{\Lambda}$ & $\varepsilon^{B}$ \\
\hline \hline 64fa $(\hat{y})$ & 0.667 & 0.458 & 0.073 & 0.041 \\
64fa $(\hat{x})$ & 0.667 & 0.258 & 0.217 & 0.018 \\
30fa $(\hat{y})$ & 0.631 & 0.384 & 0.246 & -0.007 \\
30fa $(\hat{x})$ & 0.631 & 0.296 & 0.137 & 0.098 \\
64fb $(\hat{y})$ & 0.819 & 0.698 & -0.037 & 0.054 \\
64fb $(\hat{x})$ & 0.819 & 0.452 & 0.054 & -0.120 \\
30fb $(\hat{y})$ & 0.788 & 0.638 & 0.093 & 0.033 \\
30fb $(\hat{x})$ & 0.788 & 0.485 & 0.032 & -0.032 \\
64fc $(\hat{y})$ & 0.865 & 0.630 & -0.055 & -0.050 \\
64fc $(\hat{x})$ & 0.865 & 0.464 & -0.139 & -0.069 \\
64fd $(\hat{y})$ & 0.837 & 0.566 & -0.055 & -0.047 \\
64fd $(\hat{x})$ & 0.837 & 0.255 & -0.276 & -0.200 \\
64fe $(\hat{x})$ & 0.842 & 0.393 & -0.167 & -0.091 \\
64ff $(\hat{x})$ & 0.852 & 0.516 & -0.099 & -0.022 \\
\hline
\end{tabular}

However, closer inspection of Table 6.2.11 shows that for patterns with void fraction close to 0.80 , Model A did well. Hence, a three statistic extension might still be appropriate but the coefficients of $N C P, N S I G C$ and NSIGR should be determined for each void fraction of interest rather than assuming that the ratios of the coefficients are independent of void fraction. To demonstrate this, Figure 6.2 .14 plots the absolute value of prediction error $\left(\left|K_{e q}-\hat{K}_{\mathrm{eq}}\right|\right)$ versus void fraction for both models. Data corresponding to flow in the $x$ direction across patterns 64fd is not plotted because this pattern is qualitatively different that the rest of the patterns. For this pattern, neither Model A nor Model B predict flow well because flow is almost completely blocked. Patterns $64 \mathrm{fe}$ and $64 \mathrm{ff}$ were also excluded from the plot because they are modified versions of $64 \mathrm{fd}$. 


\section{| Prediction Error}

$0.25-$

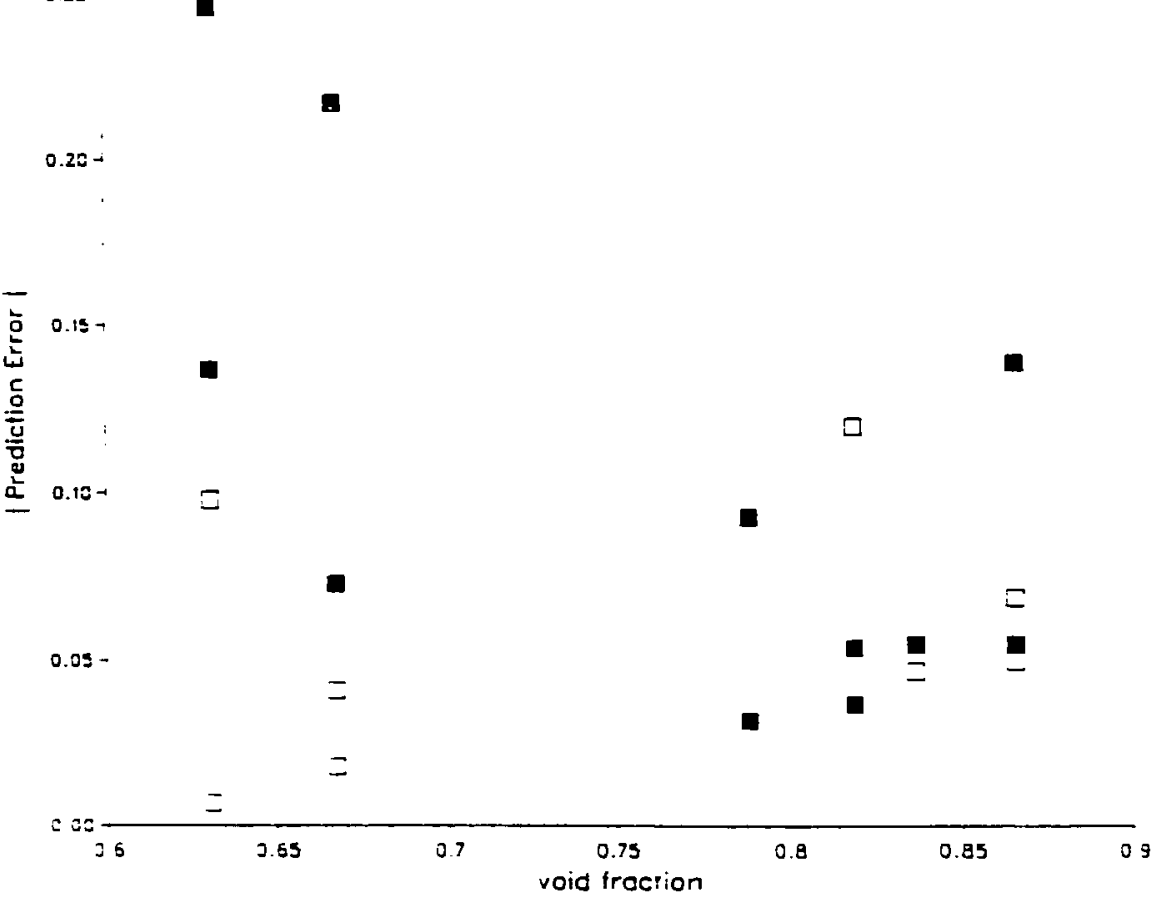

Figure 6.2.14 Magnitude of the prediction error $\left(\left|K_{e q}-\hat{K}_{e q}\right|\right)$ for Model $A$ and Model $B$ for the 30 by 30 and 64 by 64 pixel patrems simulated using fractal ideas. 


\section{Conclusion}

Both Model A

$$
\hat{K}_{\text {aq }}{ }^{\wedge}=p-[0.091 N C P+0.215 N S I G C-0.212(N S I G R-1)][1-3.67(p-.8)]
$$

and Model B

$$
\hat{K}_{e q}^{B}=p-0.327 N C P[1-0.938(p-.8)]
$$

were applied to a variety of patterns. Tables 6.2.3 and 6.2.6 show that, in general, Model B predicted flow better than Model A for the patterns simulated using the Chapter 3 model. All the pattems had void fractions between 0.60 and 0.95 and the moving average filter damping factor $(\kappa)$ which controlled the range of correlation was either $0.25,0.75$ or 1.25 (inverse pixel lengths) for each pattern. The patterns ranged in scale from 30 to 30 pixels to 128 by 128 pixels. Except for the $\kappa=0.25$ patterns, the root mean square prediction error for Model B was less than the root mean square prediction error for Model A. For the 30 by 30 clipped Gaussian process with just a few contact area features $(x=0.25)$, Model B predicted equivalent permeability less accurately for pattern $30 \times(\hat{y})$ than for the others. (Table 6.2.6).

In more detail, for the 30 by 30 pixel simulated patterns, for $\kappa=0.25,0.75$ and 1.25, $R \hat{S} E$ for Model B was $0.136,0.048$ and 0.042 (Table 6.2.6). For Model A, RŜE was $0.096,0.058$ and 0.072 for the same groups of patterns. For simulated patterns obtained by fixing $x=0.75$ and varying scale ( 30 by 30 pixels, 64 by 64 pixels and 128 by 128 pixels), $R \hat{S E}$ for Model B was $0.048,0.039$ and 0.037 . For Model A, for the same three scales, $R \dot{S E}$ was $0.058,0.044$ and 0.050 . Hence, in general, Model B predicted equivalent permeability better than Model A did.

For patte: ns simulated using fractal ideas, Model B predicted equivalent permeability much better than Model A did (Table 6.2.11, Figures 6.2.12 and 6.2.13). For all the 30 by 30 pixel and 64 by 64 pixel patterns, RSE was 0.143 for Model $A$ and 0.079 for Model B. This does not mean that a three statistic extension is invalid. In 
fact for patterns with void fractions near 0.80, Figure 6.2.14 showed that Model A did well. Rather, the method of extending the 0.80 void fraction model is suspect. To get a better extension, the coefficients of NCP, NSIGC and NSIGR should be determined for each void fraction of interest rather than assuming that the ratios of the coefficients are independent of void fraction.

For pattern $64 d(\hat{x})$, both models underestimated equivalent permeability by a factor of about two. This pattern is a special case because flow was almost completely blocked by contact area features. When passage ways were cut in the barriers so that flow could pass through more parts of the pattern, agreement between predicted and actual equivalent permeability improved. Hence, the disagreement is a consequence of predicting a flow which avoids large regions of a pattern, in terms of spatial statistics computed from all the voids and contact areas in pattern. That is, the spatial statistics were sensitive to void pixels which were isolated from the flow path. To correct for this effect, voids in regions of the pattern which are blocked off by contact area features might be viewed as contact areas. This is not pursued here, but is a future direction for research.

To close this section, Table 6.2.12 lists the void fractions of all the patterns that were used to develop and test the prediction models presented. In the table, $g_{1}$ denotes patterns simulated using the Chapter 3 model where the moving average filter decay constant is $k=0.75 . g_{2}$ denotes patterns simulated with the Chapter 3 model but with $\kappa=0.25 . \quad g_{3}$ denotes patterns simulated with the Chapter 3 model but with $\boldsymbol{\kappa}=1.25 . f_{1}$ denotes pattern simulated with fractal ideas using the first way, e.g. pattem 64fa. $f_{2}$ denotes patterns simulated with fractal ideas by means of the second way, obs denotes an observed pattern. For instance, the table indicates that equivalent permeability was computed for a 30 by 30 pixel pattern, with $60 \%$ voids, that was simulated using the Chapter 3 model with $\kappa=0.75$. 
Table 6.2.12. Summary of all the patterns studied to build and test prediction models.

\begin{tabular}{|c|c|c|c|c|}
\hline void fraction & 10 by 10 & 30 by 30 & 64 by 64 & 128 by 128 \\
\hline 0.600 & & $1^{t_{1}}$ & & \\
\hline 0.631 & & $2^{f_{1}}$ & & \\
\hline 0.650 & & $2^{81} 1^{82} 1^{83}$ & & \\
\hline 0.667 & & & $2^{f_{1}}$ & \\
\hline 0.700 & & $1^{8_{1}}$ & & \\
\hline 0.750 & & $2^{8_{1}} 1^{8_{2}} 1^{8_{3}}$ & & \\
\hline 0.768 & & & $2^{8_{1}}$ & \\
\hline 0.788 & & $2^{f_{1}}$ & & \\
\hline 0.800 & $18^{z_{1}}$ & $2 T^{3}$ & & \\
\hline 0.817 & & & & $2^{o b s} 6^{8_{1}}$ \\
\hline 0.818 & & & $2^{8_{1}}$ & \\
\hline 0.819 & & & $2^{f_{1}}$ & \\
\hline 0.826 & & & $2^{g_{1}}$ & \\
\hline 0.837 & & & $2^{f_{2}}$ & \\
\hline 0.842 & & & $1^{f_{2}}$ & \\
\hline 0.850 & & $2^{8_{1}} 1^{82} 1^{83}$ & & \\
\hline 0.852 & & & $1^{f_{2}}$ & \\
\hline 0.854 & & & $2^{8_{1}}$ & \\
\hline 0.865 & & & $2^{f_{2}}$ & \\
\hline 0.900 & & $2^{8_{1}}$ & & \\
\hline 0.950 & & $2^{81} 1^{8_{2}} 1^{83}$ & & \\
\hline
\end{tabular}




\section{ENSEMBLE VARIABILITY}

\section{Introduction and Summary}

As a extension of the main thrust of this thesis, which is to predict the equivalent permeability of a specific pattern of contact areas and voids, we give a lower bound for the standard deviation of equivalent permeability for an ensemble of simulated patterns. To clarify the notion of the ensemble, imagine that an infinitely large pattem of voids and contact areas is simulated using the Chapter 3 model. Now, partition the infinite pattern into $N$ by $N$ pixel blocks. The ensemble of $N$ by $N$ pixel patterns consists of these infinitely many $N$ by $N$ pixel blocks. In general, equivalent permeability is different for each pattern in the ensemble. Thus, the standard deviation of the equivalent permeability for the ensemble of patterns is positive for finite $N$. The standard deviation is interesting because it tells us how large a simulated pattern must be before equivalent permeability becomes very predictable.

We call the scale at which the the coefficient of variation of equivalent permeability (the ratio of the standard deviation and expected value of equivalent permeability) falls below a very small level $\varepsilon$ the scale of the representative volume (REV) scale. Hence, the smallest scale for which the lower bound for the standard deviation of equivalent permeability can fall below $\varepsilon$ is a lower bound for the REV scale.

To compute the lower bound, the expected equivalent permeability of a pattern from the ensemble is related to its void fraction. For pattems with high void fractions, a Power Average Model (Journel et al., 1986) is assumed so as to relate the void fraction of a pattern to its expected equivalent permeability. The standard deviation of void fraction for the ensemble is then expressed. With this expression, a lower bound for the standard deviation for equivalent permeability for patterns in the ensemble is derived by expanding the Power Average Model formula for expected equivalent permeability as a Taylor series in void fraction. For patterns with void 
fractions near the percolation threshold, a lower bound for the ensemble variability of equivalent permeability is derived using Percolation Theory (Broadbent and Hammersley, 1957).

We are able to use these models to relate expected equivalent permeability to void fraction because we limit attention to simulated patterns from the Chapter 3 model. In contrast, the spatial statistic models developed in Chapter 5 and 6 are intended to predict flow in terms of the spatial arrangement of voids and contact areas within a pattern for patterns simulated from the Chapter 3 model but also more general patterns. In fact, we showed in Chapter 6 that the model predicted the equivalent permeability of a variety of patterns including some simulated using fractal ideas. Furthermore, the lower bounds derived here for the variability of equivalent permeability do not account for how equivalent permeability depends on the specific arrangement of a fixed voids and contact areas within a pattern. Instead, the lower bound accounts for how the variability of void fraction leads to variability of equivalent permeability for the ensemble.

\subsection{Power Average Case}

Journel et al. (1986) developed the Power Average Model to predict the effective permeability of a sandstcine shale porous media. For a pattern consisting of a fraction $p$ of high permeability $\left(K_{k i}\right)$ regions and a fraction $1-p$ of low permeability $\left(K_{\text {low }}\right)$ regions, the Power Average Model predicts that

$$
\hat{K}_{\text {sff }}=\left(p K_{h i}^{w}+(1-p) K_{\text {low }}^{w}\right)^{\frac{1}{w}}
$$

where

$$
-1 \leq w \leq 1
$$

and $w$ depends on the spatial arrangement of the high and low permeability regions. 


\section{Application to Rock Fractures}

We apply the a Power Average Model to predict the equivalent permeability of a $N$ by $N$ pixel pattem of voids and contact areas. Each void has constant aperture. Assuming $w>0$, the Power Average model predicts that

$$
\hat{K}_{\varepsilon q}^{w}=p
$$

or

$$
\hat{K}_{2 q}=p^{1 / w}
$$

where $p$ is void fraction. To determine the parameter, $\frac{1}{w}$, the model was fit to the data from the 64 by 64 pixel patterns shown in Figure 6.1.3. Table 6.1.4 lists the equivalent permeabilities for the patterns. The best fit value of $\frac{1}{w}$ minimized

$$
\sum_{i=1}^{8}\left(\log \left(K_{e q}\right)-\frac{1}{w} \log (p)\right)^{2}
$$

The best fit to the data was

$$
\hat{K}_{\mathrm{sq}}=p^{3.43}
$$

Further,

$$
R \hat{S E}=\left[\frac{1}{7} \sum_{i=1}^{8}\left(K_{e q}-\hat{K}_{e q}\right)_{i}^{2}\right]^{\frac{1}{2}}=0.034
$$

Figure 7.1 plots the Power Average fit and actual equivalent permeability versus void fraction.

With the above relation between expected equivalent permeability and void fraction, a lower bound for the ensemble standard deviation of equivalent permeability for patterns from the statistical ensemble is derived below. Suppose that the infinite size pattern which defines the ensemble has void fraction $\langle p\rangle$ but that a particular $N$ by $N$ pixel member of the ensemble has void fraction $p$. Assuming the Taylor Series expansion 


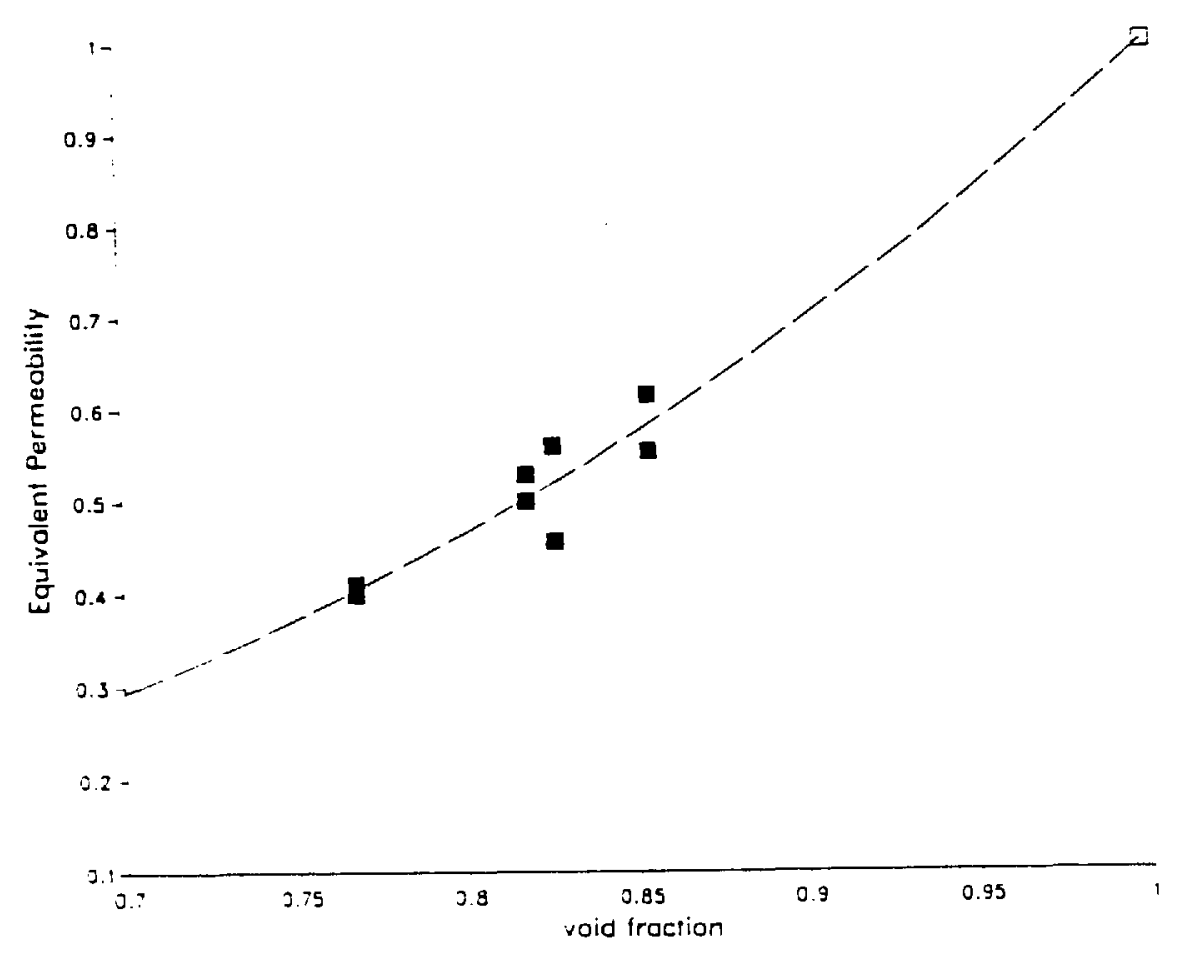

Figure 7.1 Power average model fit $\left(\hat{K}_{c q}=p^{3.43}\right)$ and actual equivalent permeability for 64 by 64 pixel patterns (Table 6.1.4). Void fraction denoted by $p$. 


$$
K_{e q}(p)-\hat{K}_{e q}\left(\langle p>)=\left.\frac{d \hat{K}_{e q}}{d p}\right|_{p>\langle p\rangle}(p-\langle p>)+\varepsilon\right.
$$

and that $\langle\varepsilon\rangle=0$ and $\langle\varepsilon(p-p\rangle)\rangle=0$, and taking the expected square value of both sides of Eq. 7.1.8 gives

$$
\sigma_{R_{e q}}^{2}=\left(\left.\frac{d \hat{K}_{e q}}{d p}\right|_{p=\propto p>} \sigma_{p}\right)^{2}+\sigma_{\varepsilon}^{2}
$$

Substituting in the derivative of $\hat{K}_{\text {eq }}$ gives

$$
\sigma_{K_{e q}}^{2} \approx\left(\frac{1}{w} p^{\frac{1}{w}}\right)^{2} \sigma_{p}^{2}+\sigma_{\varepsilon}^{2}
$$

Hence, a lower bound is

$$
\sigma_{K_{e q}}{ }^{2} \geq\left(\frac{1}{w} p^{\frac{1}{w}-1}\right)^{2} \sigma_{p}^{2}
$$

To complete the calculation, we need an expression for $\sigma_{p}$.

Estimation of $\sigma_{p}$

For $N$ by $N$ pixel simulated patterns with theoretical exponential correlograms $e^{-\lambda r}$, where $r$ is pixel lengths and $\lambda$ is inverse pixel lengths, Appendix B shows that for $N \lambda>10$,

$$
\sigma_{p}=\frac{\sqrt{\langle p\rangle(1-\langle p\rangle)}}{N} \sqrt{1+2 \pi e^{-\lambda}\left(\frac{1}{\lambda}+\frac{1}{\lambda^{2}}\right)}
$$

Further, the correlogram of a clipped Gaussian process is approximately independent of the clipping level. This independence assumption is a good approximation for clipped Gaussian processes where the clipping level leaves between $10 \%$ and $90 \%$ voids (Switzer, 1977) Hence, Eq. 7.1.12 is approximately correct for an ensemble where the average void fraction is between between $10 \%$ and $90 \%$ voids. Recall that in Chapter 3, an exponential approximation with $\hat{\lambda}=0.505$ fit the correlograms of the 128 by 128 pixel simulated patterns rather well (Figure 3.4). Thus, Eq. 7.1.12 (with $\lambda=0.505$ ) is used to approximate $\sigma_{p}$. 
Eq 7.1.12 predicts that as $\lambda$ decreases, $\sigma_{p}$ increases. This is because as $\lambda$ decreases, the spatial correlation range of the pattern increases and contact area features get bigger. If the area of a pattern is fixed, as features get bigger, the pattern becomes less homogeneous and $\sigma_{p}$ increases.

\section{Lower Bound for $\sigma_{K_{\text {eq }}}$}

To estimate a lower bound for the ensemble variability of equivalent permeability of the simulated patterns from Chapter 3, Eq 7.1.12 is substituted into Eq. 7.1.11 to get

$$
\sigma_{K_{e q}} \geq\left[\frac{1}{w}\langle p\rangle^{\frac{1}{w}}\right] \sqrt{1+2 \pi e^{-\lambda}\left(\frac{1}{\lambda^{2}}+\frac{1}{\lambda}\right)} \frac{\sqrt{\langle p>(1-\langle p\rangle)}}{N} .
$$

For patterns with fixed side length $N$, Eq 7.1.13 implies that as $\lambda$ increases, $\sigma_{\mathcal{K}_{\text {eq }}}$ decreases. Thus, equivalent permeability becomes more predictable as patterns becomes more homogeneous.

To determine how much of the variability of equivalent permeability that the lower bound predicts, we compare the lower bound and $\partial_{\kappa_{c 4}}$ for the 64 by 64 pixel patterns discussed earlier. Substituting $\langle p\rangle=0.80, \frac{1}{w}=3.43$ and $\lambda=0.505$ into Eq. 7.1.13 yields

$$
\sigma_{\kappa_{\iota 4}} \geq \frac{3.86}{N}
$$

At $N=64$, the lower bound is 0.060 . Using the data from Table 6.1 .4 , the estimated standard deviation of equivalent permeability of the simulated 64 by 64 pixels is

$$
\theta_{K_{e q}}=\left[\frac{1}{7} \sum_{i=1}^{8}\left(K_{\infty \phi}-\left\langle K_{e q}\right\rangle\right)^{2}\right]^{\frac{1}{2}}=.076
$$

Thus, the lower bound estimated a significant fraction of the total variability of equivalent permeability among the 64 by 64 pixel patterns. 


\section{Coefficient of Variation}

The coefficient of variation of equivalent permeability is the standard deviation of equivalent permeability divided by the expected value of equivalent permeability. Hence, a lower bound for the coefficient of variation is

$$
\frac{\sigma_{K e q}}{\left\langle K_{e q}\right\rangle} \geq\left[\frac{1}{w\langle p\rangle}\right] \sqrt{1+2 \pi e^{-\lambda}\left(\frac{1}{\lambda^{2}}+\frac{1}{\lambda}\right)} \frac{\sqrt{\langle p\rangle(1-\langle p\rangle)}}{N}
$$

From Eq. 7.1 .16 and using $\left\langle K_{e q}\right\rangle=.80^{3.43}=.465$, we get that

$$
\frac{\sigma_{K e q}}{\left\langle K_{e q}\right\rangle} \geq \frac{8.30}{N}
$$

Eq. 7.1.16 implies a lower bound for the REV scale. Recall that the REV scale $\left(N_{R E V}\right)$ is the scale at which the coefficient of variation drops below a very small level ع. From Eq. 7.1.16, we know that if

$$
\frac{\sigma_{K e q}}{\left\langle K_{e q}\right\rangle} \leq \varepsilon
$$

then

$$
N \geq \frac{1}{\varepsilon w} \sqrt{1+2 \pi e^{-\lambda}\left(\frac{1}{\lambda^{2}}+\frac{1}{\lambda}\right)} \sqrt{\frac{(1-\langle p\rangle)}{\langle p\rangle}}
$$

Hence, the right hand side of the above equation is a lower bound for the REV scale. That is,

$$
N_{R E V} \geq \frac{1}{\varepsilon w} \sqrt{1+2 \pi e^{-\lambda}\left(\frac{1}{\lambda^{2}}+\frac{1}{\lambda}\right)} \sqrt{\frac{(1-\langle p\rangle)}{\langle p\rangle}}
$$

The Power Average approach is valid only for patterns with high void fractions. For example, according to Eq. 7.1.6, the Power Average Model predicts a positive definite equivalent permeability for all void fractions even though experience shows that if the fraction of contact area in a simulated pattern increases beyond a critical fraction, flow is completely blocked. For pattems where flow is nearly blocked, a lower bound for the standard deviation of equivalent permeability is derived using the 
more appropriate Percolation Theory (Broadbent and Hammersley, 1957) below.

\subsection{Percolation Theory}

For an infinite pattern, if the fraction of conducting pixels is above $p_{c}$, then a infinite cluster of conducting pixels spans the infinite pattern. If the void fraction falls below $p_{c}$ no infinite cluster of conducting pixels exist. Flow across the pattern is through the infinite cluster. Percolation Theory predicts that as $p \rightarrow p_{c}$ from above,

$$
K_{e q} \rightarrow K_{o}\left(p-p_{c}\right)^{t}
$$

where $p_{c}$ is the percolation threshold, $t$ depends only on dimension (according to universality arguments) and $K_{:}$is a constant of proportionality (Kesten, 1982). In two dimensions, numerical simuations show tuat $t=1.3$. (Orbach, 1986).

As stated in the literatu e review, the way the pattern is simulated determines the percolation threshold. For instance, when square pixels are independently assigned as conducting or non-conducting, $p_{\varepsilon}=0.593$ (Orbach, 1986). For a correlated assignment scheme, Skal et al. (1973) simulated patterns of voids and pixels in a manner very similar to the way we do. Pixels were first assigned independent Gaussians. Then the Gaussians were filtered with a exponential moving average filter just like ours. They found that as range of the correlation increased, the percolation threshold moved away from 0.59 towards 0.50 . Since, our model is similar to this, we expect that our percolation threshold falls somewhere between 0.59 and 0.50 .

\section{Finite Scale}

Application of Percolation Theory to finite patterns necessitates some new vocabulary. For example, at small scales, the notion of a uniquely defined percolation threshold is not sensible. For instance, a finite region of the infinite plane might be connected by a cluster of conducting pixels even though the infinite pattern is not. Or vice versa. Hence, at finite scales, the percolation threshold is viewed as a random 
variable $p_{c}(N)$ where $N$ is the length of the pattern in pixels. We call this the empirical percolation threshold.

Not only is the threshold random, but so is the effective conductivity. The unpredictability of effective conductivity is expressed in terms of a function of the ratio of the length of the system and some characteristic length called the correlation length. In Percolation Theory, when $p>p_{c}$, the correlation length $\zeta$ is conjectured to be the effective radius of the largest cluster of non-conducting pixels (Kesten, 1987). It is conjectured (Stauffer, 1985) that

$$
\Gamma_{i} \propto\left(p-p_{c}\right)^{-v}
$$

where $v=\frac{4}{3}$ in two dimensions (Orbach, 1986). Using this correlation length, Straley (1977) conjectured that the coefficient of variation for equivalent permeability has the form

$$
\left(\frac{\sigma_{K_{\text {eq }}}}{\left\langle K_{\text {eq }}\right\rangle}\right)^{2} \propto\left[\frac{\zeta}{N}\right]^{2}
$$

provided that the pattern side length $N$ exceeded the correlation length $\zeta$.

\section{Simulated Patterns}

To express a lower bound for the coefficient of variation for equivalent permeability for an ensemble of finite simulated patterns, Eq 7.2.2 is rewritten as

$$
K_{a q}=K_{o}\left(p-p_{c}(N)\right)^{t}+\varepsilon
$$

where $\varepsilon$ is the difference between the equivalent permeability of a pattern and the Percolation Theory prediction for equivalent permeability. We assume that $K_{o}$ is a constant and that $t=1.3$. The next step is to relate the variance of $p$ and $p_{c}(N)$ to the variance of equivalent permeability.

To do this, an expression for the standard deviation of empirical threshold is needed. Stauffer (1985) derives a general formula for the standard deviation of the 
empirical percolation threshold for a $N$ by $N$ pixel patterns using scaling arguments. The formula is

$$
\sigma_{p_{c}}(N)=B N^{-\frac{1}{v}}
$$

Further, Levinshtein et al. (1976) studied fluctuations of the empirical percolation threshold for independent site percolation in two dimensions and fit the following formula

$$
\sigma_{p_{c}(N)}=0.54(N+1.4)^{-\frac{1}{v}}
$$

For our simulated patterns, $\sigma_{P_{c(N)}}$ implicitly depends on the correlogram. We use Eq. 7.2.5 without evaluating $B$.

Taylor Series

Assuming a Taylor Series expansion for $K_{e q}$

$$
\begin{gathered}
K_{\alpha q}\left(p, p_{c}(N)\right)-\hat{K}_{e q}\left(\left\langlep>,\left\langle p_{c}(N)>\right)=\right.\right. \\
\left.(p-p>) \frac{d \hat{K}_{\alpha q}}{d p}\right|_{p><p>}+\left(p_{c}(N)-\left\langle p_{c}(N)>\right) \frac{d \hat{K}_{e q}}{\left.d p_{z}(N)\right|_{p_{c}(N) \propto p_{c}(N)>}+\varepsilon^{\prime},}\right.
\end{gathered}
$$

and taking the expected square average of both sides gives

$$
\sigma_{K_{c q}}^{2}=\left(\sigma_{p}^{2}+\sigma_{\rho_{c}(N)}{ }^{2}\right)\left(K _ { 0 } \left(\left\langle p>-\left\langle p_{c}(N)>\right)^{t-1}\right)^{2}+\sigma_{\varepsilon}{ }^{2} .\right.\right.
$$

To derive the above, cross terms in $\varepsilon^{\prime}$ were assumed to average to zero. Dropping the $\sigma_{\varepsilon}^{2}$ term gives

$$
\sigma_{K_{c \varphi}}{ }^{2} \geq\left[( 1 + 2 \pi e ^ { - \lambda } [ \frac { 1 } { \lambda ^ { 2 } } + \frac { 1 } { \lambda } ] , \frac { \langle p > ( 1 - p \rangle ) } { N ^ { 2 } } + \frac { B ^ { 2 } } { N ^ { \frac { 2 } { v } } } ] \left[\left\lfloorK _ { 0 } \left(\left\langle p>-\left\langle p_{c}(N)>\right)^{t-1}\right]^{2} .\right.\right.\right.\right.
$$

Assuming that $\left\langle K_{e q}\right\rangle=K_{0}\left(\langle p\rangle\left\langle p_{c}(N)>\right)^{t}\right.$, a lower bound for the coefficient of variation is 


$$
\left(\frac{\sigma_{K_{e q}}}{\left\langle K_{e q}\right\rangle}\right)^{2} \geq\left[\left(1+2 \pi e^{-\lambda}\left[\frac{1}{\lambda^{2}}+\frac{1}{\lambda}\right]\right) \frac{\langle p\rangle(1-\langle p\rangle)}{N^{2}}+\frac{B^{2}}{N^{\frac{2}{v}}}\right] t^{2}\left[\left(\langle p\rangle-\left\langle p_{\varepsilon}(N)\right\rangle\right)\right]^{-2}
$$

The lower bound includes terms which explicitly depend on the correlogram via $\lambda$ as weli as terms which implicitly depend on the correlogram such as $\left\langle p_{c}(N)>, B\right.$ and perhaps $\boldsymbol{K}_{o}$. Although further work is needed to evaluate these terms before the formula can be applied, its limiting behavior is clear. For patterns with fixed side length, as the average void fraction of the ensemble of patterns approaches the percolation threshold, the coefficient of variation of equivalent permeability and hence the REV scale diverges. Note that in Straley's expression (Eq. 7.2.2), as void fraction approaches the percolation threshold, the coefficient of variation also diverges.

\section{Summary}

A lower bound for the coefficient of variation of equivalent permeability for an ensemble of patterns was expressed for cases where the void fraction of a pattern is large enough so that the Power Average Model is valid. As the length of patterns in the ensemble increases, the lower bound for coefficient of variation tends to a small quantity. For the case where the void fraction is near the percolation threshold, a lower bound for the coefficient of variation of equivalent permeability was estimated using Percolation Theory. As the expected void fraction of a pattern in the ensemble approached the expected empirical percolation threshold, the lower bound for the coefficient of variation of equivalent permeability diverged. For both cases, as the spatial correlation range of the sinulated patterns increased, the lower bound for the REV scale increased. 


\section{Variable Aperture Patterns}

\section{Introduction and Summary}

In Chapters 5 and 6 , the equivalent permeability of a pattern of contact areas and voids with constant apertures was predicted in terms of spatial statistics computed from the arrangement of the voids and contact areas within the pattern. How to apply the model to patterns where voids have variable apertures is the subject of this Chapter. Using the constant aperture model, two lower bounds for the equivalent permeability of variable aperture patterns are given. As an illustration, both bounds are compared to actual equivalent permeability fo: two 30 by 30 pixel simulated pattenis with variable apertures.

The first lower bound for the equivalent permeability of a variable aperture pattern is obtained by first transforming all apertures. In the transformation, all apertures below an adjustable cutoff are set to zero and all apertures above the cutoff are set to the cutoff. Since each transformed aperture is less than or equal to each of original aperture, the flow through the original pattem is greater than or equal to the flow through the transformed pattern. This is because local hydraulic conductivity is proportional to aperture cubed in our flow model. Hence, the model prediction for the equivalent permeability of the transformed pattern provides a lower bound for the equivalent permeability of the original pattem. The second lower bound is obtained by setting all the apertures above the cutoff to a intermediate value between the cutoff and the maximum aperture in the pattern. The intermediate value chosen is the cube root of the harmonic average of the cube of apertures above the cutoff. A heuristic argument based on circuit theory rules for finding the maximum resistance of a network of variable resistors motivated this second bound. 


\section{First Lower Bound}

The first lower bound is based on an approach described by Kirkpatrick (1978) and motivated by work by Ambekagor (1971). Kirkpatrick describes how to estimate a lower bound for the effective conductivity of a system where local conductivity varies continuously, using Percolation Theory which is designed to work for systems where conductivities take one of two values-- zero or a constant. To get the lower bound, all conductances below a cutoff are set to zero and all conductances above the cutoff are equated to the cutoff. The Percolation Theory estimate of the effective conductivity of the transformed system is a lower bound for the effective conductivity of the original system. Since each cutoff gives a different lower bound, the best choice of cutoff is the one that gives the largest lower bound.

Following the spirit of the above idea, a lower bound for the equivalent permeability of a variable aperture pattern is es imated below. To get the first lower bound, the apertures of the variable aperture pattern are transformed as follows

$$
b(i, j)= \begin{cases}b_{c u} & \text { for } b(i, j) \geq b_{\text {cur }} \\ 0 & \text { for } b(i, j)<b_{\text {cur }}\end{cases}
$$

where $b(i j)$ is the aperture of the $(i j)^{\text {th }}$ pixel and $b_{c u t}$ is an adjustable cutoff. Since the new apertures are everywhere less than or equal to the original apertures, the flow across the transformed pattem is less than or equal to the total flow across the original pattern.

The flow through the original pattern is related to its equivalent permeability $K_{e q}$ as follows

$$
Q=\Delta P \frac{b_{\max }^{3}}{12 \mu} K_{e q}
$$

where $b_{\max }$ is the largest aperture in the pattern, $\Delta P$ is the pressure drop applied and $\mu$ is fluid viscosity. By similar arguments, the flow through the transformed pattern is related to the equivalent permeability of the transformed pattem $K_{\mathrm{eq}}{ }^{T}\left(b_{c u}\right)$ as 


$$
Q^{T}\left(b_{c w s}\right)=\Delta P \frac{b_{c w}{ }^{3}}{12 \mu} K_{e q}{ }^{T}\left(b_{c w}\right)
$$

Since, $Q \geq Q^{T}\left(b_{c s}\right)$ for all choices of $b_{c u s}$,

$$
K_{a q} \geq \frac{Q^{T}}{Q_{\max }}
$$

and

$$
K_{e q} \geq\left[\frac{b_{c u}}{b_{\max }}\right]^{3} K_{e q}{ }^{T}\left(b_{c u}\right)
$$

Although all choices of $b_{c \ll}$ give lower bounds for $K_{a q}$, the best choice of $b_{c w s}$ gives the largest of the lower bounds. This largest lower bound is closest to the equivalent permeability of the pattern. Next, the best lower bound and actuai equivalent permeability are computed for two simulated patterns.

\section{Application to Simulated Patterns}

Both simulated pattems are modifications of the same realization of a Gaussian process which is shown in Figure 8.1. This same realization was clipped to get the patterns shown in Figure 6.1.1. Each pixel gets an aperture as follows:

$$
b(i, j)=\frac{e^{X Y(i, j)}}{e^{X Y_{\max }}}
$$

where $Y(i, j)$ denotes the Gaussian assigned to the $(i, j)^{t_{k}}$ pixel and $Y_{\max }$ is the largest Gaussian in the pattern. The broadness of the aperture distribution is controlled by $\chi$. For pattem $8 a, \chi=0.50$, for pattern $8 b, \chi=0.75$. Hence, pattem $8 b$ has a broader distribution of apertures than does pattern $8 a$. Figure 8.2 shows the cumulative aperture histograms for each pattern. Because of the normalization, the maximum aperture in each pattern is unity.

\section{Equivalent Permeability}

For each pattern, equivalent permeability is computed using the methods described in Chapter 4. Tables 8.1 and 8.2 show that computed equivalent 


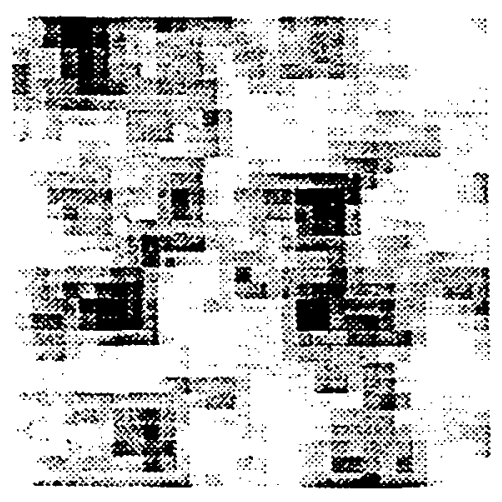

XBL $893-1094$

Figure 8.1 Pattem of correlated Gaussians. Darker pixels correspond to larger Gaussians. 
Cumulative histograms for aperture

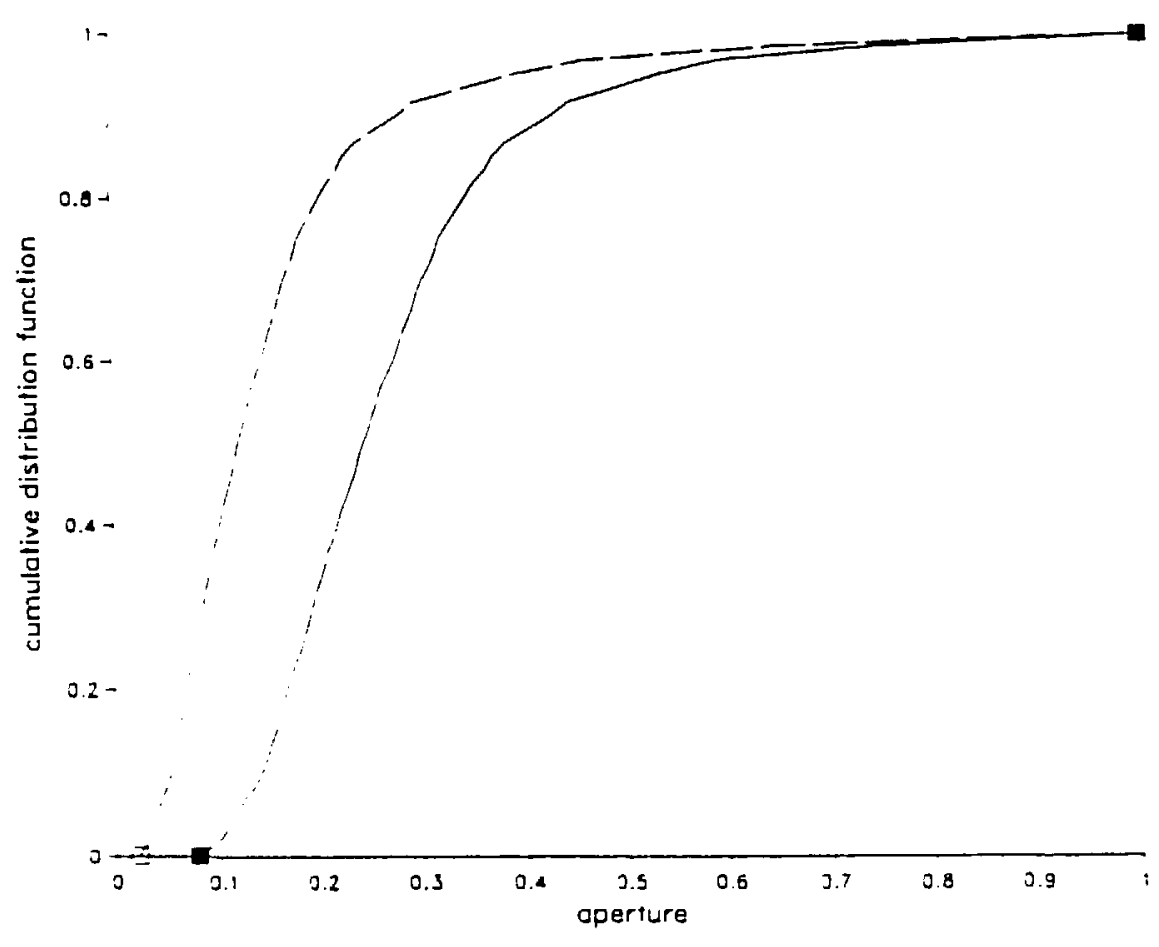

XBL 893-1095

Figure 8.2 Cumulative aperure histograms for patterns $8 a$ and $8 b$. 
permeability stabilized as mesh size increased.

Table 8.1. Equivalent permeability versus mesh size for variable aperture pattern 8a.

\begin{tabular}{|ccc|}
\hline mesh & iterations & $K_{\text {eq }}$ \\
\hline \hline 30 by 30 & 8000 & 0.0140 \\
60 by 60 & 6000 & 0.0153 \\
90 by 90 & 4000 & 0.0159 \\
120 by 120 & 2000 & 0.0157 \\
150 by 150 & 2000 & 0.0159 \\
\hline
\end{tabular}

Table 8.2. Equivalent permeability versus mesh size for variable aperture pattern $8 b$.

\begin{tabular}{|ccc|}
\hline mesh & ierations & $K_{\text {aq }}$ \\
\hline \hline 30 by 30 & 8000 & 0.00159 \\
60 by 60 & 6000 & 0.00175 \\
90 by 90 & 4000 & 0.00195 \\
120 by 120 & 2000 & 0.00193 \\
150 by 150 & 2000 & 0.00196 \\
\hline
\end{tabular}

Next, the lower bounds are computed and compared to the above equivalent permeabilities. 


\section{Lower Bounds for Simulated Patterns}

Since, both patterns are transformations of the same realization of Gaussian process, the estimated equivalent permeability of the transformed patterns $\hat{K}_{\text {eq }}^{T}$ is the same function for both patterns. With $p\left(b_{c w}\right)$ denoting the fraction of voids with aperture greater than or equal to $b_{c w}$, the estimate of a lower bound is

$$
K_{e q} \geq b_{c u t}{ }^{3} \hat{K}_{e q}{ }^{T}\left(p\left(b_{c u s}\right)\right)
$$

where Model B from Chapter 6 is used to estimate the equivalent permeability of the transformed pattern as follows

$$
\hat{K}_{\text {aq }}{ }^{T}\left(p\left(b_{\text {cus }}\right)\right)=p\left(b_{c u s}\right)-0.327 N C P\left[1-0.938\left(p\left(b_{c u s}\right)-0.80\right)\right]
$$

In Figures 8.3 and $8.4, \hat{K}_{e q}{ }^{T}\left(p\left(b_{c u}\right)\right), b_{c u s}{ }^{3}$ and their product which is the first lower bound, are plotted versus $p\left(b_{\text {cus }}\right)$ for each pattern.

For each pattern, the value of the cutoff that maximized the first lower bound was found by adjusting $b_{c w}$. We call the value of $b_{c w}$ which maximizes the first lower bound $b^{\circ}$. The maximum value of the first lower bound is denoted as $\hat{K}_{a q}{ }^{\circ}$. The fraction of voids with aperture above $b^{*}$ is called $p^{*}$. Table 8.3 lists $b^{*}, p^{*}, \hat{K}_{e q}{ }^{*}$ and the ratio of $\hat{K}_{\text {eq }}{ }^{\bullet}$ and $K_{\text {eq }}$ for both patterns. Table 8.3 shows that the discrepancy between the lower bound and actual equivalent permeability was greater for pattern $8 b$ which had a broader distribution of apertures. In general, as the aperture distribution narrows, the bound should approach actual equivalent permeability. Next, a lower bound which comes significantly closer to the equivalent permeability of the simulated patterns is given. 

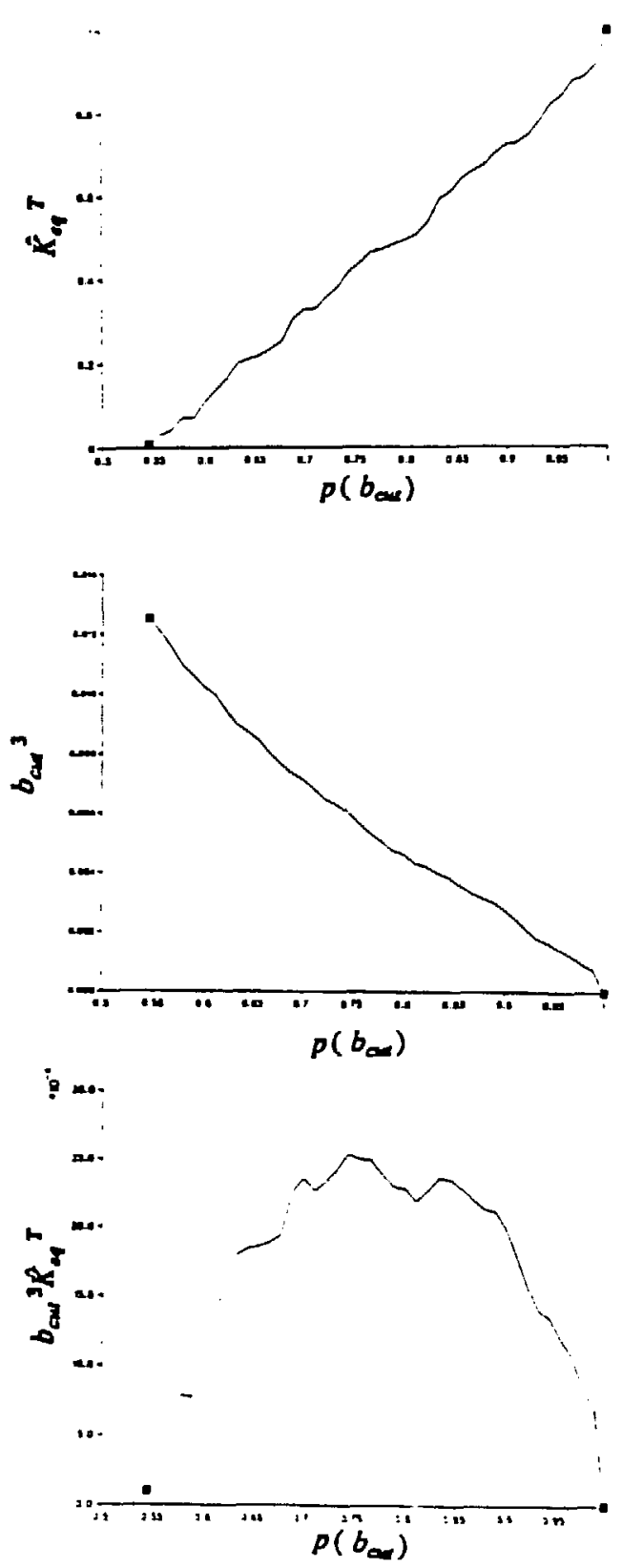

Figure 8.3 To get the first lower bound for pattern 8a, all apertures below an adjustable cutoff $\left(b_{\mathrm{cm}}\right)$ are set to zero. All apertures above the cutoff are set to the cutoff. Top plot is estimated equivalent permeability $\left(K_{e q}^{T}\right)$ of transformed pattern versus the fraction $\left(p\left(b_{\mathrm{cu}}\right)\right)$ of apertures above the adjustable cutoff $b_{c u}$. Middle plot is $b_{c u}{ }^{3}$ versus $p\left(b_{c u}\right)$. Bottom plot is $K_{c q}^{\top} b_{c a}{ }^{3}$ versus $p\left(b_{c a w}\right)$. The best lower bound is the maximum of the bottom curve. 

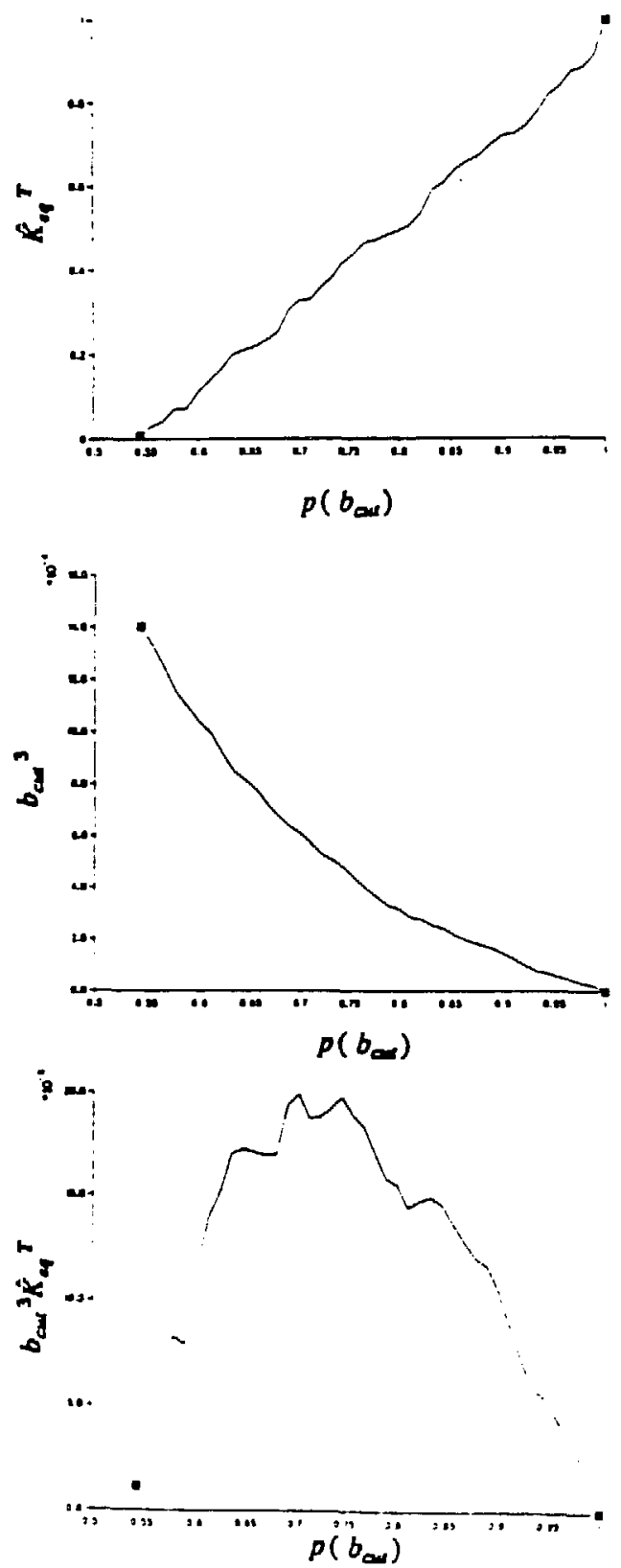

XBL 89$]-1042$

Figure 8.4 Same as Figure 8.3 but for pattem $8 \mathrm{~b}$ rather than pattern $8 \mathrm{a}$. 
Table 8.3. First lower bound for the equivalent permeability of variable aperture patterns.

\begin{tabular}{|cccccc|}
\hline & $b^{*}$ & $p^{*}$ & $\hat{K}_{\text {eq }}{ }^{*}$ & $K_{\text {eq }}$ & $\frac{\hat{K}_{\text {eq }}}{K_{\text {eq }}}$ \\
\hline \hline $8 a$ & 0.183 & 0.745 & $2.5610^{-3}$ & $1.5910^{-2}$ & 0.161 \\
$8 b$ & 0.085 & 0.699 & $2.0110^{-4}$ & $1.9610^{-3}$ & 0.103 \\
\hline
\end{tabular}

\section{Second Lower Bound}

The second lower bound is a modified version of the first. The idea is based on work by Charlaix et al. (1987). They applied Percolation Theory to patterns with variable conductances by modifying the approach of Kirkpatrick (1978). Instead of setting all conductances above the cutoff to the cutoff, conductances above the cutoff were set to the harmonic average of conductances over the cutoff. They justified this assignment by arguing that the conducting regions in their system acted like electrical resistors in series.

The justification for such an assignment is based on the lower bound for the effective permeability of a porous medium derived by Beran (1968). Recall from Chapter 2 that the equivalent permeability of a porous medium is bounded below by the harmonic average of the local permeabilities. To illustrate when equivalent permeability takes the lower bound, consider the pattern shown below in Figure 8.5. Apertures of equal value appear as the same color.

For a pressure drop in the $x$ direction, the local cubic law model equates the flow across the $i^{\text {th }}$ stripe to

$$
Q_{i}=1 \frac{\frac{\Delta P_{i}}{\Delta x_{i}}}{12 \mu} b_{i}{ }^{3}
$$

where $l$ is the length of the pattern, $\Delta P_{i}$ is the pressure drop across the $i^{\text {th }}$ stripe, $\Delta x_{i}$ is the width of the $i^{\text {th }}$ strip and $b_{i}$ is the aperture in the $i^{\text {th }}$ stripe. Hence, each stripe has 

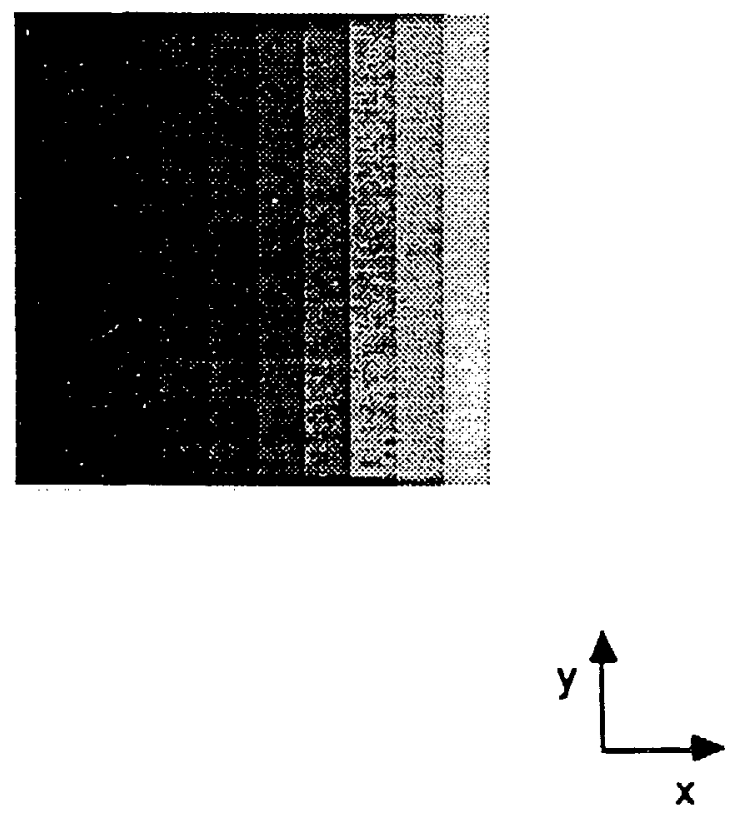

XBL 893-1096

Figure 8.5 Variable aperture pattern. Equal apertures appear as same color. 
hydraulic resistance proportional to the inverse of aperture cubed. Since the striped pattern can be viewed as a series arrangement of hydraulic resistors, the overall resistance of the pattern is proportional to the average of inverse aperture cubed. This is the hydraulic equivalent of the electrical circuit rule which equates the overall resistance of resistors in series to the sum of the resistances. Hence, overall flow is proportional to the harmonic average of aperture cubed. Thus,

$$
K_{a q}=\frac{Q}{Q_{\max }}=\left(\frac{1}{b_{\max }}\right)^{3}<b^{-3}>^{-1}
$$

where the harmonic average of aperture cubed is $\left\langle b^{-3}\right\rangle^{-1}$.

Assuming the validity of the cubic law flow model, Eq. 8.13 exactly predicts the equivalent permeability of the pattern shown in Figure 8.6 and also serves as a lower bound for the equivalent permeability of more general patterns.

Note that if a contact area strip is introduced as shown below in Figure 8.6, equivalent permeability drops by a factor of $p$ where $1-p$ is the fraction of contact area in the pattern. The equivalent permeability of the striped pattern is

$$
K_{\text {sq }}=\left(\frac{1}{b_{\max }}\right)^{3}<b^{-3}>^{-1} p
$$

This example suggests the following lower bound for more general patterns.

$$
K_{c q} \geq\left(\frac{1}{b_{\max }}\right)^{3}<b^{-3} ; b_{c u s}>^{-1} \hat{K}_{c q}{ }^{\top}\left(b_{c u}\right)
$$

where $\left\langle b^{-3} ; b_{c u}\right\rangle^{-1}$ is the harmonic average of aperture cubed for apertures above the cutoff $b_{e u s}$. We have no proof that the above is a general lower bound for general patterns. In fact, special cuses exist for which it will overestimate flow. For instance, suppose that a void is off the flow path and hence has no influence on flow. If the aperture of this void off the flow path is large enough, Eq. 8.15 will overestimate flow. For future research, it would be wise to identify such pixels and treat them as contact areas. 

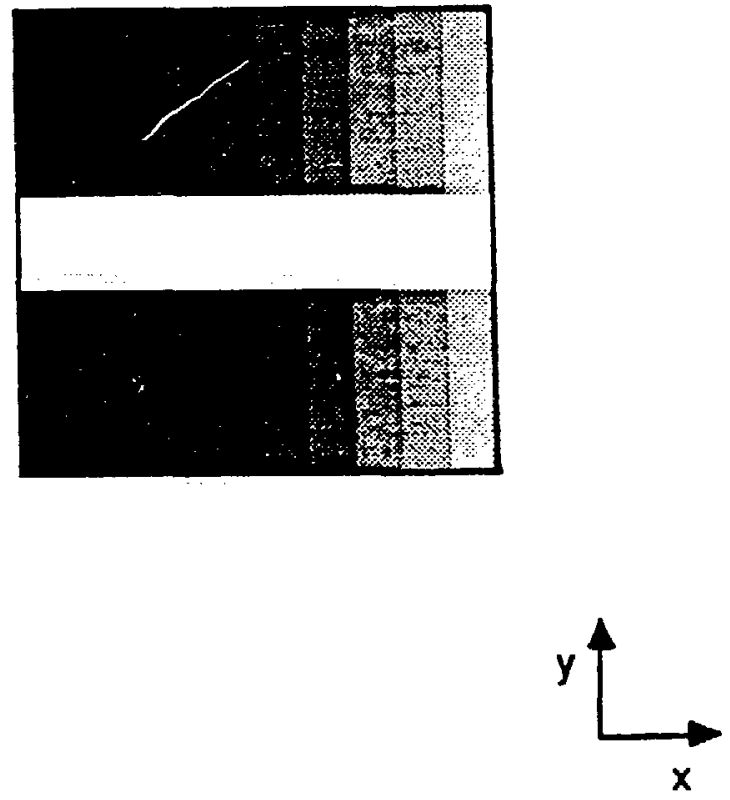

XBL 893-1097

Figure 8.6 Same pattern as in Figure 8.5 except for contact area strip in the middle of the pattem. Equal apertures appear as same color. 
To interpret this as a modification of the first lower bound, consider the aperture transformation

$$
b(i, j)= \begin{cases}\left\langle b^{-3} ; b_{c a s}\right\rangle^{\frac{1}{3}} & \text { for } b(i, j) \geq b_{c a s} \\ 0 & \text { for } b(i, j)<b_{c a d}\end{cases}
$$

The second lower bound is an estimate for the equivalent permeability of such a transformed pattern. Next, the second lower bound is compared to the actual equivalent permeability of pattems $8 a$ and $8 b$.

\section{Application to Simulated Patterns}

In Figures 8.7 and $8.8, \hat{K}_{e q}^{T}\left(p\left(b_{c u t}\right)\right),<b^{-3}, b_{c u t}>^{-1}$ and their product which is the second lower bound, are plotted versus $p\left(b_{\text {our }}\right)$ for both patterns. For each pattem, the value of the cutoff that maximized the second lower bound was found by adjusting $b_{c u s}$. The value of $b_{\text {cus }}$ which maximizes the second lower bound is called $b^{* *}$. The maximum value the second lower bound takes is denoted by $\hat{K}_{\text {eq }}{ }^{*}$. The fraction of voids with aperture above $b^{* *}$ is called $p^{*}$. Table 8.3 lists $b^{* *}, p^{* *}, \hat{K}^{* r}$ and the ratio of $\hat{K}^{*{ }_{a q}}$ and $K_{\text {eq }}$ for both patierns. Again, the agreement between the lower bounds and actual equivalent permeability was better for the pattern with the nanower range of apertures.

Table 8.4. Second lower bound for the equivalent permeability of variable aperture patterns.

\begin{tabular}{|c|c|c|c|c|c|}
\hline & $b^{*}$ & $p$ & $\tilde{K}_{a \varphi}$ & $K_{e q}$ & $\frac{\dot{K}_{e q} \ddot{\mid}}{K_{e q}}$ \\
\hline $8 a$ & 0.153 & 0.854 & $8.2810^{-3}$ & $1.5910^{-2}$ & 0.521 \\
\hline $8 b$ & 0.074 & 0.763 & $8.2410^{-4}$ & $1.96 \mathrm{IG}^{-3}$ & 0.420 \\
\hline
\end{tabular}



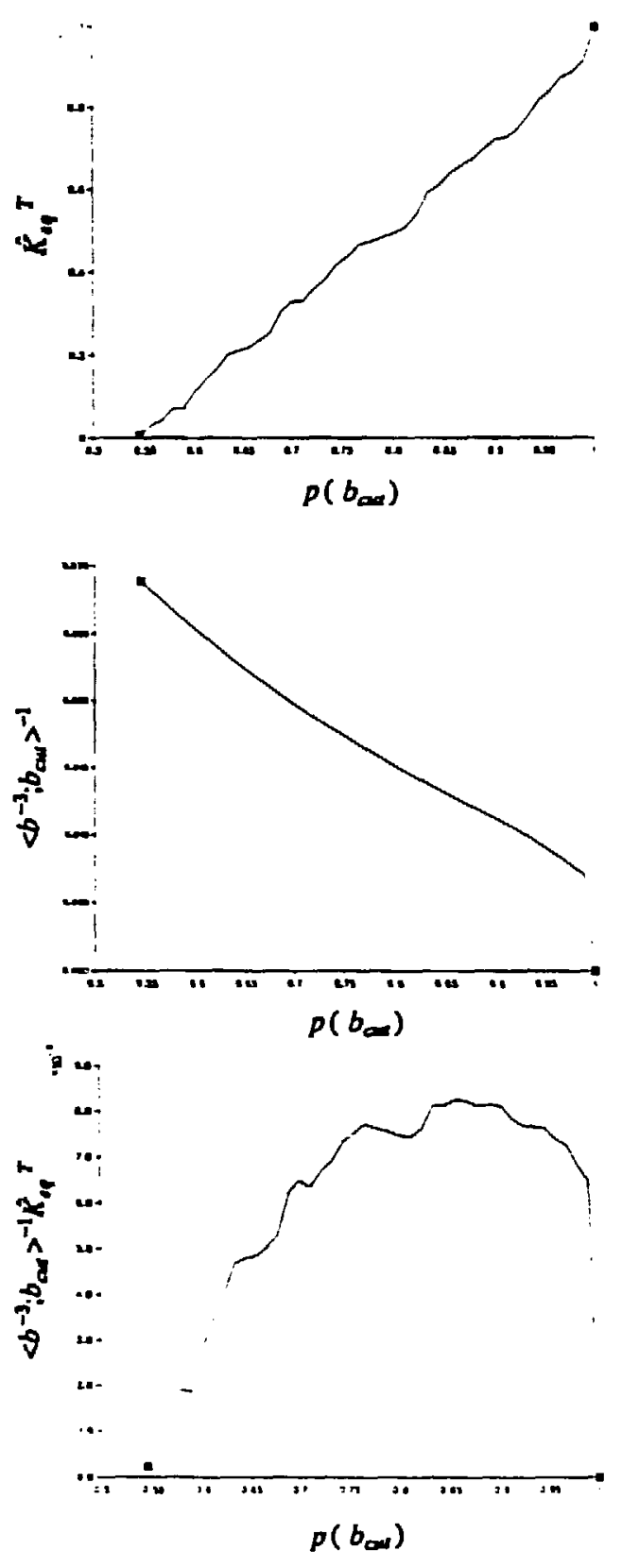

Figure 8.7 To get the second lower bound for pattem 8a, all apertures below an adjustable cutoff $\left(b_{\mathrm{cus}}\right)$ are set to zero. The rest of the apertures are equated to harmonic average of apertures above the cutoff. From the top, $\left(K_{\text {eq }}^{T}\right)$, harmonic average of apertures above the cutoff, and the their product which is the second lower bound. The best lower bound is the maximum of the bottom curve. 

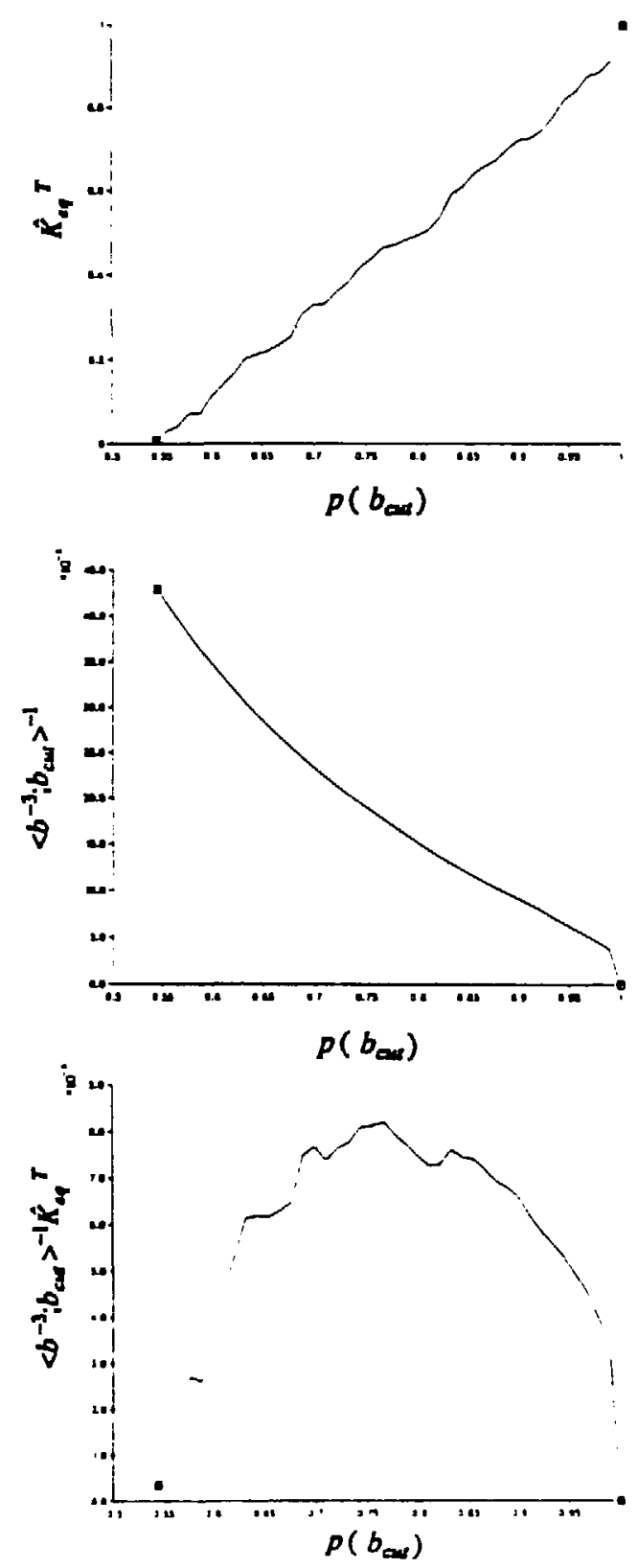

XBZ 893-1044

Figure 8.8 Same as Figure 8.7 but for pattem $8 \mathrm{~b}$ instead of pattem $8 \mathrm{a}$. 


\section{Summary}

The equivalent permeability of a variable aperture pattern depends on not only the arrangement of voids and contact areas within the pattern, but the apertures assigned to the void pixels. Hence, the information necessary to estimate equivalent permeability is much more complicated than the information necessary to estimate the equivalent permeability of a pattern where voids have constant apertures. By transforming a variable aperture pattern into a binary aperture pattern where apertures are zero or equal to a constant, lower bounds for equivalent permeability were presented using the prediction model developed in Chapter 5 and 6 . In both lower bounds, all apertures below a chosen cutoff were equated to zero. In the first lower bound, all apertures above an adjustable cutoff were equated to the cutoff. In the second lower bound, all apertures above the cutoff were equated to the cube root of the harmonic average of the cube of apertures that were above an adjustable cutoff. Analysis of two simulated patterns showed that the second lower bound was closer to equivalent permeability than was the first. The agreement between the lower bound and equivalent permeability was better for the pattern with the narrower range of apertures. These lower bounds are a first step in developing spatial statistics to estimate the equivalent permeability of a variable aperture pattern. 


\section{CONCLUSION}

In this thesis, the normalized flow through a fracture, i.e. the equivalent permeability of a fracture, was predicted in terms of spatial statistics computed from the arrangement of voids and contact areas within the fracture.

Chapter 3 showed how patterns of voids with constant aperture and contact areas were simulated. The simulated patterns had complexity typical of experimental data. Chapter 4 detailed the local cubic law flow model and a finite-difference method was used to solve the equations for flow for each pattern. Based on the analysis of 10 by 10 and 30 by 30 pixel simulated patterns of voids (with constant aperture) and contact areas with $80 \%$ voids, a model to predict equivalent permeability was presented in Chapter 5.

In Section 5.2, equivalent permeability was predicted in terms of the change point statistic $(N C P)$ which measures how often pixel alternate from void to contact area, or vice versa, in the rows of the pattern which are parallel to the applied pressure drop across the pattern. The fitted model parameters were not sensitive to scale; they were almost the same when fitting the model to either the 10 by 10 pixel patterns alone or the 30 by 30 pixel patterns alone. In Section 5.3, equivalent permeability was predicted in terms of row and column dispersion statistics (NSIGR and NSIGC) which measured how dispersed pixels are in rows and columns of the patterm. In Section 5.4, equivalent permeability was also predicted in terms of a statistic (NBREAK) which measured the width of the flow path between contact area features. Unlike for the other statistics, the fitted parameters for the NBREAK model were significantly different for the 10 by 10 and 30 by 30 pixel patterns. In Section 5.5, equivalent permeability was predicted in terms of a linear combination of NCP, NSIGC and NSIGR. The model parameters were well determined.

In Chapter 6, the $N C P$ model was extended to patterns with other void fractions. In general, for a variety of patterns from the Chapter 3 model which ranged in scale 
from 30 by 30 pixets to 128 by 128 pixels, the extended model predicted equivalent permeabiiity well. Further, the extended model accurately predicted the equivalent permeability of patterns simulated by shanging the range of correlation in the Chapter 3 model. The extended model also did a reasonable job of predicting the equivalent permeability of patterns simulated using fractal ideas. (Table 6.2.11) However, for one of the inhomogeneous patterns simulated using fractal idcas (64d), the model underestimated equivalent permeability by a factor of about two (Table 6.2.9). For this case, flow across the pattern was almost entirely blocked. A extended model involving NCP, NSIGC and NSIGR did not predict equivalent permeability as well as the extended NCP model. We speculate that this is because the method of extension was too simplistic for the three statistic model.

How well the prediction model works for more complex patterns or pattems simulated in other different ways is an open research question. Even if the model does not work for more complex patterns of voids and contact areas, there may be ways of still using it. For instance, suppose a pattern of voids and contact areas is so complex that flow can not be compured using the computer. It may be possible to simplify the pattern by partioning it into 30 by 30 pixel blocks and then estimating a $x$ and $y$ direction equivalent permeability for each block using our model. Hence, the 900 bits of information contained by each block (each pixel is contact or voids) would be reduced to two bits of information. A future research direction is to determine how to combine the estimated equivalent permeabilities for each 30 by 30 pixel blocks so as to estimate the flow through the whole pattern.

Alternatively, the model may be useful for predicting the effect of selected contact area features on flow. For instance, the influence of the largest contact area features in a complex pattern flow might be predicted by applying the prediction model to a transformed version of the original pattern. In such a transformation, all contact area features smaller than a certain size might be transformed into voids so as 
to isolate the effect the largest features have on flow.

The major point of this thesis is to predict the equivalent permeability of a specific pattern of voids and contact areas. To complement this effort, a lower bound for the variation of equivalent permeability for an ensemble of simulated patterns from the Chapter 3 model was given in Chapter 7. To get the lower bound, both the Power Average Model (Journel et al. 1986) and Percolation Theory (Broadbent and Hammersley, 1957) were used to relate the void fraction of a pattern to its expected equivalent permeability. As the size of a simulated pattern increased, the lower bounds approached zero. In terms of these lower bounds, a lower bound for the REV scale was derived.

In Chapter 8, a lower bound for the equivalent permeability of a variable aperture pattern was given. The $N C P$ model from Chapter 6 which is valid for patterns where voids have constant aperture, was applied to transformed versions of variable aperture patterns. Two lower bounds were given. In the first transformation, all apertures above a chosen cutoff were set to the cutoff; all apertures below the cutoff were set to zero. In the second transformation, instead of setting apertures above the cutoff to the cutoff, apertures were equated to the cube root of the harmonic average of the cube of apertures above the cutoff. For two simulated patterns, the second lower bound came much closer to actual equivalent permeability (Tables 8.3 and 8.4). The discrepancy between the bound and actual equivalent permeability was less for the pattern with the narrower range of apertures. These lower bounds for equivalent permeability are a first step in the development of a model to predict the equivalent permeability of a variable aperture pattern. 


\section{APPENDIX A}

The average run length of visd pixels in the rows of a simulated pattem with isotropic correlogram $\rho(r)$, where $r$ is lag in pixel lengths, is shown to be

$$
<R U N>=\frac{1}{(1-p)(1-\rho(1))}
$$

For simulated patterns with 0.80 void fractions, and $\rho(1)=e^{-.505},\langle R U N>\approx 12.6$ pixels.

\section{Derivation}

Two void pixels belong to the same run of voids if no contact area pixel lies between them. Consider a long row of $N$ pixels from a simulated pattern. Partition the row into distinct runs of voids and contact areas. From Section 5.2, the fraction of pairs of pixels which alternate from void to contact or contact to void is $C P$. Hence the number of pairs which alternate from contact to void is $N \frac{C P}{2}$. By Eq. 5.2.6,

$$
\frac{C P}{2}=p(1-p)(1-\rho(1))
$$

where $\rho(1)$ is the correlogram (Journel and Huijbregts, 1978) at lag 1. Hence, the average number of runs of voids is $N p(1-p)(1-\rho(1))$. Since the average number of void pixels is $N p$, the average run length of void pixels is

$$
<R U N>=\frac{N p}{N p(1-p)(1-\rho(1))}=\frac{1}{(1-p)(1-\rho(1))}
$$

From Chapter 3 (Eq. 3.8), $\rho(1)=e^{-505}$ for our simulated patterns. Hence, for simulated patterns with 0.80 void fractions, $\triangle R U N>=12.6$ pixels. 


\section{APPENDIX B}

The standard deviation of the void fraction for an ensemble of $N$ by $N$ pixel simulated pattems, with exponential correlogram

$$
\rho(r)=e^{-\lambda r},
$$

and average void fraction, $\langle p>$, is well approximated as follows

$$
\sigma_{p}^{2}=\frac{s p>(1-\langle p>)}{N^{2}}\left[1+2 \pi e^{-\lambda}\left(\frac{1}{\lambda}+\frac{1}{\lambda^{2}}\right)\right]
$$

\section{Derivation}

Let $I_{i j}$ be the indicator function for the $i j^{\text {th }}$ pixel.

$$
I_{i j}= \begin{cases}1 & \text { when } i j^{\text {th }} \\ 0 & \text { when } i j^{\text {th }} \text { pixel is void } \\ & \text { is contact area }\end{cases}
$$

The void fraction of a simulated pattern is

$$
p=\frac{1}{N^{2}} \sum_{i=1}^{N} \sum_{j=1}^{N} I_{i j}
$$

The variance of $p$ is

$$
\sigma_{p}^{2}=\left\langle p^{2}\right\rangle-\langle p\rangle^{2}
$$

From Eqs. B.4 and B.5,

$$
\sigma_{p}^{2}=\left[\frac{1}{N^{4}} \sum_{i=1}^{N} \sum_{j=1}^{N} \sum_{k=1}^{N} \sum_{l=1}^{N} E\left(I_{i j} I_{k l}\right)\right]-\left\langle p>^{2}\right.
$$

By the definition of the correlogram (Eq. 3.6 in Chapter 3)

$$
\sigma_{p}{ }^{2}=\frac{1}{N^{4}}\left[\sum_{i=1}^{N} \sum_{j=1}^{N} \sum_{k=1}^{N} \sum_{l=1}^{N}\langle p\rangle^{2}+\langle p>(1-\langle p\rangle) \rho(d(i j, k l))]-\langle p\rangle^{2}\right.
$$

where the distance $d$ between the $(i, j)^{\text {th }}$ and $(k, l)^{\text {th }}$ pixels equals

$$
d(i j, k l)=\sqrt{(i-k)^{2}+(j-l)^{2}} .
$$


The $\langle p\rangle^{2}$ term in the quadruple sum contributes $\langle p\rangle^{2}$ and exactly cancels the $-\langle p\rangle^{2}$ to the right of the quadruple sum. Hence,

$$
\sigma_{p}^{2}=\frac{\langle p>(1-\langle p\rangle)}{N^{4}} \sum_{i=1}^{N} \sum_{j=1}^{N} \sum_{k=1}^{N} \sum_{l=1}^{N} \rho(d(i j, k l))
$$

The quadruple sum has $N^{4}$ terms. For each of the $N^{2}$ pixels in the pattern, there is double sum involving $N^{2}$ correlograms terms. Each double sum is approximated as $1+\int_{r=1}^{\frac{N}{2}} 2 \pi \rho(r) r d r$. In this approximation, the discrete double sum of correlogram terms is replaced by a integral within a circular region with radius $\frac{N}{2}$. That is,

$$
\sum_{i=1}^{N} \sum_{j=1}^{N} \sum_{k=1}^{N} \sum_{i=1}^{N} \rho(d(i j k l))=N^{2}\left(1+\int_{r=1}^{\frac{N}{2}} 2 \pi \rho(r) r d r\right)
$$

Hence,

$$
\sum_{i=1}^{N} \sum_{j=1}^{N} \sum_{k=1}^{N} \sum_{=1}^{N} \rho(d(i j k l))=N^{2}\left[1+2 \frac{\pi}{\lambda}\left(e^{-\lambda}-\frac{N}{2} e^{-\lambda \frac{N}{2}}\right)+2 \frac{\pi}{\lambda^{2}}\left(e^{-\lambda}-e^{-\lambda \frac{N}{2}}\right)\right]
$$

For For $N \lambda>10$, some of the boundary terms vanish and the above can be approximated as

$$
\sum_{i=1}^{N} \sum_{j=1}^{N} \sum_{k=1}^{N} \sum_{l=1}^{N} \rho(d(i j k l))=N^{2}\left[1+2 \pi e^{-\lambda}\left(\frac{1}{\lambda}+\frac{1}{\lambda^{2}}\right)\right]
$$

Collecting terms we have,

$$
\sigma_{p}^{2}=\frac{\langle\varphi>(1-\langle\varphi\rangle)}{N^{2}}\left[1+2 \pi e^{-\lambda}\left(\frac{1}{\lambda}+\frac{1}{\lambda^{2}}\right)\right]
$$

For $\lambda=0.505$, the approximation should be good for $N>20$ because then $N \lambda>10$. Figure B.1 plots Eq. B.13 and a numerical evaluation of Eq. B.9 for $\lambda=.505$ $<p>=0.80$. As expected, Eq. B. 13 is a good approximation to Eq. B. 9 when $N>20$. 


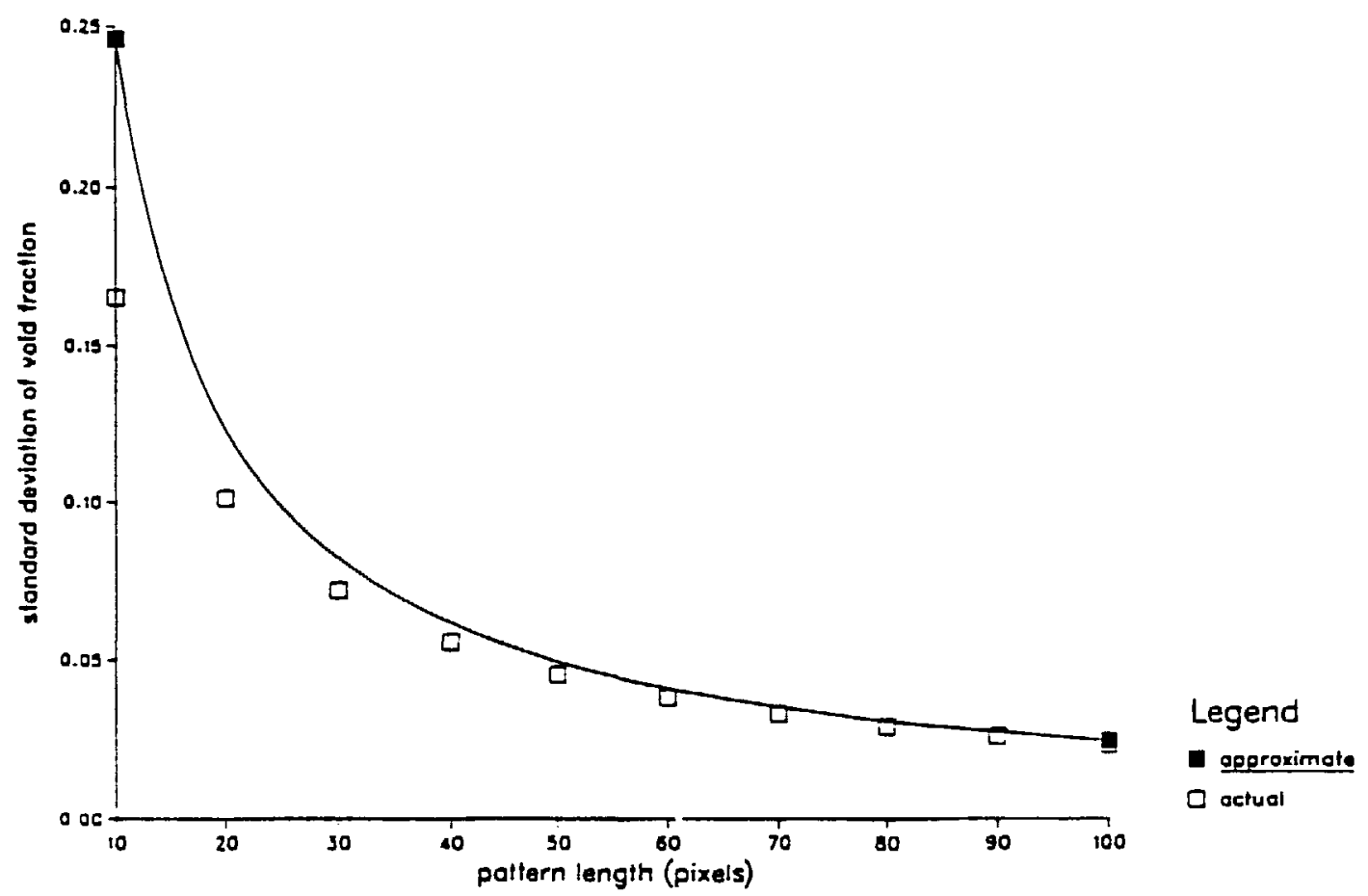

XBL $895-1677$

Figure B.1 Approximation and exact expression for standard deviation of void fraction as a function of pattern side length for a simulated pattern with exponential correlogram. Here, correlogram is taken to be $e^{-2 r}$ where $r$ is lag in pixel lengths and $\lambda$ is taken to be 0.505 (inverse pixel lengths). 


\section{REFERENCES}

Ambegakor, V., B. I. Halperin and J. S. Langer, Hopping Conductivity in Disordered Systems, Phys. Rev. B., Vol 4, p. 2612, 1971.

Beran, M. J., Statistical Continuum Theories, Interscience, New York, 1968.

Berryman, J. G. and S. C. Blair, Use of a Digital Image Analysis to Estimate Fluid Permeability of Porous Materials. Application of Two-Point Correlation Functions, J. Appl. Phys. 60, pp. 1930-1938, 1986.

Broadbent S. and Hammersley J., Percolation processes. I. Crystals and mazes. II. Proc. Cambridge Philos. Soc. 53, pp. 629-641 and 642-645, 1957.

Brown, S., Flow Through Rock Joints: The Effect of Slurface Roughness, $J$. Geophys. Res., 92, pp. 1337-1347, 1987.

Charlaix, E., E. Guyon and S. Roux, Permeability of a Random Array of Fractures of Widely Varying Apertures, Transport in Porous Media, Vol. 2, No. 1, February 1987.

Chen, D. Zimmerman, R., and Cook, N., The Effect of Contact Area on the Permeability of Fractures, To be presented at 30th U.S. Symposium on Rock Mechanics, Morgantown, WV. June, 1989.

Coakley, K., Muralidhar, K., Long, J.C.S and Myer, L., Equivalent Permeability of Statistically Simulated Single Fractures, Proceedings of the Conference on Geost in:stical, Sensitivity, and Uncertainty Methods for Ground-Water Flow and Radionuclide Transport Modeling, San Francisco, CA, September 15-17. 1987, Edited by Bruce E. Puxton, Battele Memorial Institute, Columbus Divison, Columbus, Ohio. pp. 441-469, 1989.

Desbarats, A., Stochastic Modeling of Flow in Sand-Shale Sequences, Ph.D. Thesis, Stanford University, 1986.

Deutsch, C., Calculating Effective Absolute Permeability in Sandstone/Shale Sequences, submitted to $J$. of Pet. Tech., 1988.

Hildebrand, F.B., Introduction to Numerical Analysis, McGraw-Hill Book Company, Second Edition, New York, 1974.

Holman, J.P. , Heat Transfer, McGraw-Hill Book Company, Fourth Edition, New York, 1976.

Journel, A. G., C. D. Deutsch and A. J. Desbarats, Power Averaging for Block Effective Permeability, SPE 15128, 1986. 
Journel, A.G. and C. Huijbregts, Mining Geostatistics, Academic Press, London, 600 pp., 1978.

Journel, A. G. and E. H. Isaaks, Conditional Indicator Simulation: Application to a Saskatchewan Uranium Deposit, Math Geol., Vol. 16, No. 7, pp. 685-718, 1984.

Kesten, H. Percolation Theory for Mathematicians, Birkhauser, Boston, 1982.

Kesten, H. Percolation and the first-passage percolation. The Annals of Probability, Vol. 15, No. 4 pp 1231-1271, October, 1987.

Kirkpatrick, S., Ill-Condensed Matter, edited by R. Balian, R. Maynard and G. T. Toulouse, North-Holland, Amsterdam, 1979.

Kirkpatrick, S., Percolation and Conduction, Reviews of Modern Physics, Vol. 45, No. 4, pp. 574-588, 1973.

Levinshtein, B, Shk'ovskii, M., Efros A, The relation between the critical exponent of percolation theory, Sov.Phys. JETP, Vol. 42, No. 1, pp. 197-200, 1976.

Mandelbrot, B, Th2 Fractal Behavior of Nature, Freeman, New York, 1983.

Mood, A.M., Grabill, F.A., Boes, D.C., Introduction to the Theory of Statistics, McGraw-Hill, Inc., New York, 1974.

Muralidhar, K. and J.C.S Long, A Scheme for Calculating Flow in Fractures using Numerical Grid Generation in Three-Dimensional Domains of Complex Shapes, LBL-24453, 1987.

Nolte, D. D., L. J. Pyrak-Nolte, and N. G. W. Cook, The Fractal Geometry of the Flow Paths in Natural Fractures in Rock and the Approach to Percolation, accepted for publication in Special Issues on Fractals in Geophysics in PAGEOPH. 1987.

Orbach, R., Dynamics of Fractal Networks, Science, Vol. 231, pp. 814-819, 1986.

Pike, G. and Seager, C., Percolation and conductivity:A computer study.I. Phys. Rev. B. 10, pp. 1421-1434, 1974.

Pyrak-Nolte, L. J., L. R. Myer, N. G. W. Cook and P. A. Witherspoon, Hydraulic and Mechanical Properties of Natural Fractures in Low Permeability Rock, presented at Sixth International Conference on Rock Mechanics, Montreal, Canada, August 30, 1987.

Rothman D., Cellular-Automaton Fluids: A Model for Flow in Porous Media, Geophysics, Vol. 53 No 4., pp. 509-518, 1988. 
Schlichting, H., Boundary-Layer Theory, McGraw Hill, New York, 1979.

Serra, J, Image Analysis and Mathematical Morpholcgy Academic Press, New York, 1982.

Shklovskii, B., Efros, A, Electronic Properties of Doped Semiconductors, SpringerVerlag, Berlin, Heidelberg, New York and Tokyo, 1984.

Skal, A, Shklovskii, B, Eíros A, Percolation level in a two-dimensional random potential. Sov. Pnys.-Solid State 15, 961, 1973.

Stauffer, D., Introduction to Percolation Theory, Taylor and Francis, Philadelphia and New York, 1985.

Stoll, E. and Domb C., Shape and size of two-dimensional percolation clusters with and without correlation, J. Phys. A:Marh. Gen., Vol 12, No. 10, 1979.

Strang, G. Linear Algebra, Academic Press, N.Y. 1980.

Switzer, P, Estimation of Spatial Distributions from Point Sources with Application to Air Pollution Measurement, International Statistical Institute, Vol. 47, pp. 121-147, 1977.

Tsang, Y., The Effect of Tortuosity on Fluid Flow Through a Single Fracture, Water Resources Research, Vol. 20, No. 9, Fp. 1209-1215, 1984.

Walsh, J, Effect of Pore Pressure and Confining Pressure on Fracture Permeability, International Journal of Rock Mechanics, Mineral Science and Geomechanics, Vol. 18, pp. 429 to $435,1981$. 